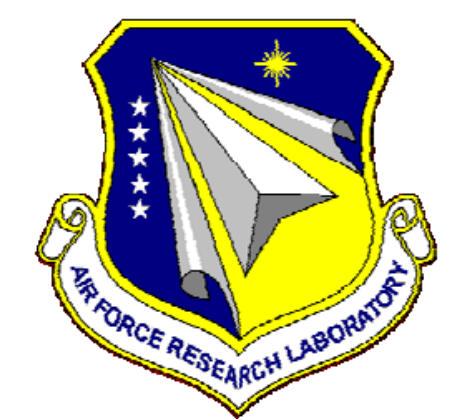

\title{
AFRL-RX-WP-TR-2015-0066
}

\section{QUANTUM CONFINED SEMICONDUCTORS}

Gail J. Brown

AFRL/RXAN

FEBRUARY 2015

Final Report

Distribution Statement A. Approved for public release; distribution unlimited.

See additional restrictions described on inside pages

\section{STINFO COPY}

AIR FORCE RESEARCH LABORATORY

MATERIALS AND MANUFACTURING DIRECTORATE

WRIGHT-PATTERSON AIR FORCE BASE, OH 45433-7750

AIR FORCE MATERIEL COMMAND

UNITED STATES AIR FORCE 


\section{NOTICE AND SIGNATURE PAGE}

Using Government drawings, specifications, or other data included in this document for any purpose other than Government procurement does not in any way obligate the U.S. Government. The fact that the Government formulated or supplied the drawings, specifications, or other data does not license the holder or any other person or corporation; or convey any rights or permission to manufacture, use, or sell any patented invention that may relate to them.

Qualified requestors may obtain copies of this report from the Defense Technical Information Center (DTIC) (http://www.dtic.mil)

AFRL-RX-WP-TR-2015-0066 HAS BEEN REVIEWED AND IS APPROVED FOR PUBLICATION IN ACCORDANCE WITH ASSIGNED DISTRIBUTION STATEMENT.

//SIGNED//

GAIL J. BROWN, Program Manager

Nanoelectronic Materials Branch

Functional Materials Division
DIANA M. CARLIN, Chief

Nanoelectronic Materials Branch

Functional Materials Division

//SIGNED//

KAREN R. OLSON, Deputy Chief

Functional Materials Division

Materials \& Manufacturing Directorate

This report is published in the interest of scientific and technical information exchange and its publication does not constitute the Government's approval or disapproval of its ideas or findings. 


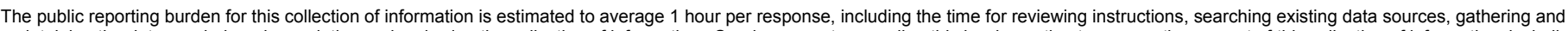

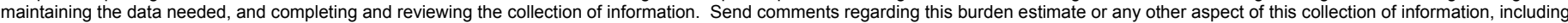

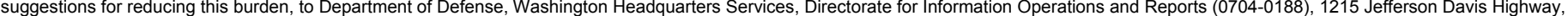

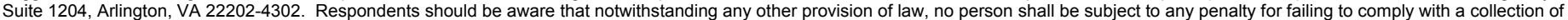
information if it does not display a currently valid OMB control number. PLEASE DO NOT RETURN YOUR FORM TO THE ABOVE ADDRESS.

\begin{tabular}{|c|c|}
\hline $\begin{array}{c}\text { 1. REPORT DATE (DD-MM-YY) } \\
\text { February } 2015\end{array}$ & 2. REPORT TYPE \\
\hline $\begin{array}{l}\text { 4. TITLE AND SUBTITLE } \\
\text { QUANTUM CONFINED SEMICONDUCTORS }\end{array}$
\end{tabular}

Final

3. DATES COVERED (From - To)

Final

8 June 2011 - 16 January 2015

5a. CONTRACT NUMBER

In-House

bb. GRANT NUMBER

5c. PROGRAM ELEMENT NUMBER

61102F

6. AUTHOR(S)

Gail J. Brown

5d. PROJECT NUMBER

2305

5e. TASK NUMBER

5f. WORK UNIT NUMBER

X091 (PS113200)

7. PERFORMING ORGANIZATION NAME(S) AND ADDRESS(ES)

AFRL/RXAN

8. PERFORMING ORGANIZATION REPORT NUMBER

3005 Hobson Way

Wright-Patterson AFB, OH 45433

9. SPONSORING/MONITORING AGENCY NAME(S) AND ADDRESS(ES)

Air Force Research Laboratory

Materials and Manufacturing Directorate

Wright-Patterson Air Force Base, OH 45433-7750

Air Force Materiel Command

United States Air Force

10. SPONSORING/MONITORING

AGENCY ACRONYM(S)

AFRL/RXAN

11. SPONSORING/MONITORING AGENCY REPORT NUMBER(S)

AFRL-RX-WP-TR-2015-0066

12. DISTRIBUTION/AVAILABILITY STATEMENT

Distribution Statement A. Approved for public release; distribution is unlimited.

13. SUPPLEMENTARY NOTES

Approved by 88ABW Public Affairs Office: Case number 88ABW-2015-1223 on 19-MAR-2015. Report contains color.

\section{ABSTRACT}

This overall objective of this research task was to push forward the state-of-the-art in utilizing semiconductor quantum dots in optical and optoelectronic devices of interest to the Air Force. The full potential of semiconductor quantum dots cannot be achieved by random formation and assembly processes. For narrow optical linewidths, suppressed thermal excitation, and tailored dot-to-dot interactions, the quantum dot size, composition and location needs to be precisely controlled to engineer the desired properties. Two complementary but distinct methods for fabricating uniform quantum dots with controlled dot/nanoparticle size, composition and location are proposed. 1) Nanopatterning and annealing of MBE grown planar InAs. 2) Synthesis of semiconductor nanocrystals by Supercritical Fluid CO2 process. In addition to the fabrication process development, a key objective is to model and characterize the fundamental physics and chemistry of these nanoscale heterostructures to provide the firm scientific basis for these materials to transition into advanced devices, such as detectors, lasers, optical communication and quantum computing.

15. SUBJECT TERMS

electro-optical, nanoelectronics, nanoparticles, quantum dots

16. SECURITY CLASSIFICATION OF:

\begin{tabular}{|l|c|c|}
\hline $\begin{array}{c}\text { a. REPORT } \\
\text { Unclassified }\end{array}$ & $\begin{array}{c}\text { b. ABSTRACT } \\
\text { Unclassified }\end{array}$ & $\begin{array}{c}\text { c. THIS PAGE } \\
\text { Unclassified }\end{array}$ \\
& & \\
\end{tabular}

17. LIMITATION OF ABSTRACT:

SAR
18. NUMBER OF PAGES

113 19a. NAME OF RESPONSIBLE PERSON (Monitor) Gail J. Brown 19b. TELEPHONE NUMBER (Include Area Code) (937) 255-9854 


\section{TABLE OF CONTENTS}

$\underline{\text { Section }}$ Page

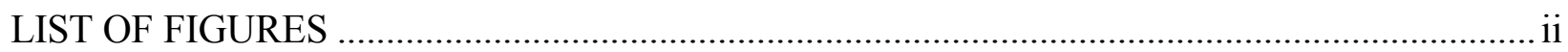

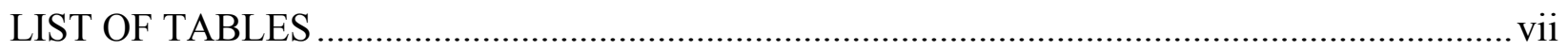

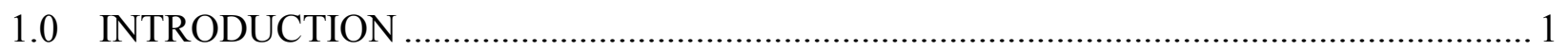

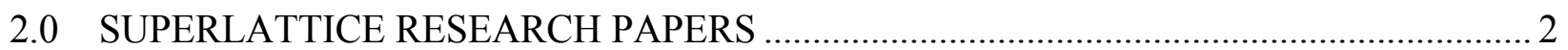

2.1 Interface Roughness Estimate from Carrier Transport in InAs/GaSb

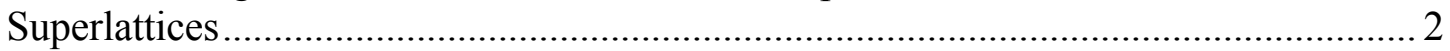

2.2 Control of Residual Background Carriers in Undoped Mid-infrared InAs/GaSb Superlattices....................................................................................... 11

2.3 Type-II Superlattice Materials Research at the Air Force Research Laboratory

2.4 Phase Sensitive Detection of Photoluminescence with Fourier

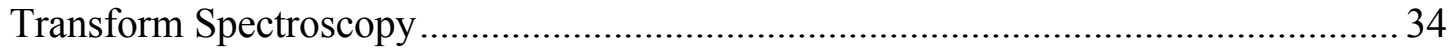

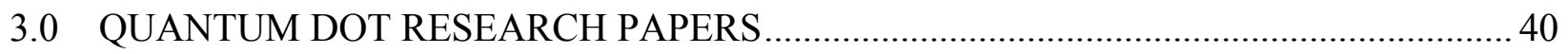

3.1 TEM Characterization of InGaAs QDIP Structures...................................................... 40

3.2 Photoluminescence of PbS Quantum Dots on Semi-Insulating GaAs ......................... 45

3.3 Surface, Crystallographic and Optical Properties of PbS QDs Formed

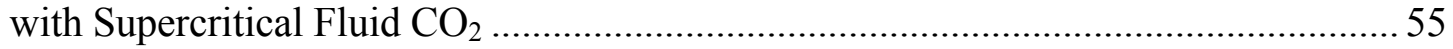

3.4 Lead Sulfide Quantum Dot Synthesis, Deposition, and Temperature Dependence Studies of the Stokes Shift.................................................................... 71

3.5 Emission of Precipitation Deposited PbS Quantum Dots on

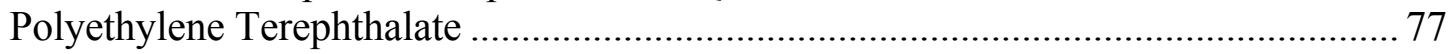

3.6 Stability Studies of Lead Sulfide Colloidal Quantum Dot Films on

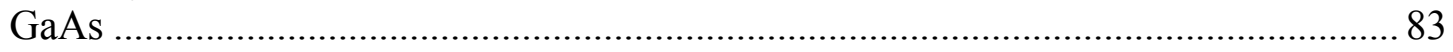

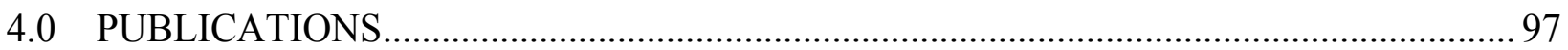

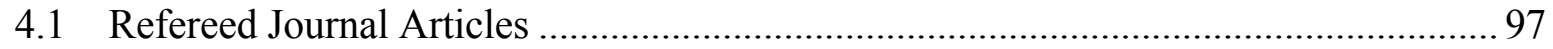

4.2 Conference Proceedings ......................................................................................... 99

LIST OF ACRONYMS, ABBREVIATIONS, AND SYMBOLS............................................ 102

Distribution Statement A. Approved for public release; distribution unlimited. 


\section{LIST OF FIGURES}

Figure

Page

1 Mobilities as a function of temperature in the temperature regime below the type conversion. ( $\boldsymbol{\Delta}:$ SL5, $\square:$ SL4, $\bullet:$ SL3, ०:SL2, $\bullet: S L 1$ )

2 The layer width dependence of the measured mobilities (filled circles) together with the best power law fit (solid curve).

3 The scattering integral (dimensionless) $F\left(\Lambda, k_{F}\right)$, Equation (10), as a function of the product $\Lambda k_{F}$ (dimensionless), where $\Lambda$ is the roughness correlation length and $k_{F}$ is the Fermi wave vector. Here, the screening factor $S_{C}=1$.

4 Photoresponse spectrum of the $0.5 \mu$ m-thick $21 \AA$ InAs/ $24 \AA \mathrm{GaSb}$ superlattices (SLs) taken at $10 \mathrm{~K}$. The insert shows the $100 \mu \mathrm{m} \times 100 \mu \mathrm{m}$ scan by an atomic force microscope of the SL sample.

5 The temperature dependence of (a) the carrier concentrations, (b) resistivity as a function of $1000 / \mathrm{T}$ for the $0.5 \mu \mathrm{m}$-thick $21 \AA$ InAs/24 $\AA$ GaSb superlattices $(\bullet)$ on the GaSb substrate and the GaSb substrate $(\Delta)$. Below $20 \mathrm{~K}$, holes in the GaSb substrate show hopping conduction, which is an artifact and not reliable.

6 (a) The carrier density $(\bullet)$ and (b) in-plane carrier mobility ( $\boldsymbol{\Delta}$ ) comparing SL samples grown at different growth temperatures. The insert shows the 3D AFM images of 20x20 $\mu \mathrm{m}$ area scan of the SLs grown at 370 (top) and $430{ }^{\circ} \mathrm{C}$ (bottom) showing significant differences in surface smoothness.

$7 \quad$ (a) The carrier density (•), (b) in-plane carrier mobility ( $\boldsymbol{\Delta})$; comparing SL samples with and without post-annealing. All SL samples had same design of $21 \AA$ InAs/24 $\AA$ GaSb superlattices.

8 The carrier density $(\bullet)$ and in-plane mobility $(\boldsymbol{\Delta})$ of the $21 \AA$ $(\mathrm{InAs}+\mathrm{IF}) / 24 \AA(\mathrm{GaSb}+\mathrm{IF})$ superlattices with four variations of interface shutter sequences; InSb/GaAs, InSb/InSb, GaAs/GaAs, and GaAs/InSb comparing with those of uncontrolled sequence $0 / 0$.

9 (a) The Hall mobility and (b) PL intensity of $21 \AA$ InAs/24 $\AA$ GaSb superlattices (SLs) grown on old (blue) and new buffer (red). The Hall mobility and PL intensity of the SLs grown on the new buffer that was grown at gradually decreasing temperatures of $490-400{ }^{\circ} \mathrm{C}$ were twice as large as for the SL grown on the old $\mathrm{GaSb}$ buffer that was entirely grown at $490{ }^{\circ} \mathrm{C}$.

10 The $5 \mathrm{~K}$ photoluminescence spectra of $21 \AA \mathrm{InAs} / \mathrm{X} \AA \mathrm{GaSb}$ superlattices, where $\mathrm{X}=24,36$ and $48 \AA$.

11 Calculated energy InAs/GaSb Superlattice Band Gap as a Function of InAs Layer Width for a Fixed GaSb Width of $24 \AA$ .23

ii

Distribution Statement A. Approved for public release; distribution unlimited. 
12 Comparison of the Normalized Photoluminescence and Photo-

Conductivity Spectra for the Same Series of Samples where the InAs

Width is Varied from 21 to $40 \AA$, while the GaSb Width was kept constant

at $26 \AA$

13 Secondary Ion Mass Spectroscopy Depth Profiles of Two Superlattice

Samples with Different Buffer Layer Growth Conditions. Panel a) is our

Standard GaSb Buffer Layer Process, b) is the more Gradual Transition from the Buffer Layer Growth Temperature to the Superlattice Growth

Temperature. Key Differences are Highlighted by the Dashed Ovals.

14 Comparison of the Photoresponse Spectra from 4 Samples with Varying InAs Width. Arrows indicate which Intensity Axis Applies to the Spectra.

15 Photoresponse Intensity, $100 \mathrm{meV}$ above the band edge, for spectra shown in Figure 14, as a function of the measured band gap energy. The star marks the results for an improved sample.

16 Calculated Oscillator Strength of the Optical Transition across the Superlattice Band Gap as a Function of InAs Width....

17 Sheet Carrier Density at 10K Measured by Hall Effect for a SLs with varied InAs Width and Fixed GaSb Width of $24 \AA$ Á....

18 X-ray Rocking Curve for InAs (48 Á)/GaSb (21 Á) Superlattice with a Measured Period of $68.5 \AA$ and Lattice Mismatch Strain of $-0.56 \%$.

19 Cross-sectional High Resolution TEM Image of InAs/GaSb (48 Á/20 Á) Superlattice Showing the First Two Periods in the Vicinity of the GaSb (100) Substrate.

20 Comparison of the Photoresponse Spectra of Two InAs (21 Á)/GaSb (48 Å) Superlattice Samples with Different Interface Processes. The weaker spectrum is the same as shown in Figure 14.

21 Comparison of various InSb:Te PL spectra : (a) PL intensity measured with PSD, (b) PL intensity measured without lock-in amplifier, and (c) shows the same measurement as spectrum (a) but with 1000 scans rather than 100 scans. Indicated are the parameters - BOMEM scan speed, lockin time constant, and scan speed - used for the measurements. Spectrum (b) is recorded with the default scanning speed of $0.5 \mathrm{~cm} / \mathrm{s}$.

22 Comparison of InSb:Te PL spectra recorded with PSD at various lock-in time constant and roll off settings: (a) $300 \mu \mathrm{s}, 24 \mathrm{~dB}$, (b) $300 \mu \mathrm{s}, 12 \mathrm{~dB}$, (c) $1 \mathrm{~ms}, 12 \mathrm{~dB}$, and (d) $3 \mathrm{~ms}, 12 \mathrm{~dB}$. All spectra were recorded with a BOMEM scan speed of $0.02 \mathrm{~cm} / \mathrm{s}$.

23 Demonstration of the impact of the BOMEM scan speed variation on the PSD of the InSb:Te emission: (a) $0.02 \mathrm{~cm} / \mathrm{s}$ and (b) $0.05 \mathrm{~cm} / \mathrm{s}$.

24 InSb:Te PL spectra measured with PSD using various lock-in time constants. As indicated, besides the time constant, the remaining parameters were the same for all spectra.

iii

Distribution Statement A. Approved for public release; distribution unlimited. 
25 Diffraction patterns representing the two beam imaging conditions used for (a) $\mathrm{G}=200$ and (b) $\mathrm{G}=022$ bright-field and dark-field images.

26 Cross-sectional TEM images of the single-layer QDIP structure showing

(a) $\mathrm{G}=(022)$ bright-field image and (b) (200) dark-field image.

27 Cross-sectional TEM images of the 5-layer QDIP structure showing (a)

$\mathrm{G}=(022)$ bright-field image and (b) (200) dark-field image.

28 Cross-sectional TEM images of the 10-layer QDIP structure showing (a) $\mathrm{G}=(022)$ bright-field image and (b) (200) dark-field image.

29 Cross-sectional TEM images of the 20-layer QDIP structure showing the (200) dark-field image.

30 Plan-view TEM bright-field $(\mathrm{G}=(220))$ image of the single-layer QDIP structure.

31 Plan-view TEM bright-field $(\mathrm{G}=(220))$ image of the 10-layer QDIP structure with the top GaAs-contact layer.

32 Plan-view TEM bright-field $(\mathrm{G}=(220))$ image of the 20-layer QDIP structure with the top GaAs-contact layer removed.

33 Plan-view TEM bright-field $(\mathrm{G}=(220))$ image of the 10-layer QDIP structure with the top GaAs-contact layer removed.

34 SEM image of the quantum dots. The bar on the right hand side corresponds to $50 \mathrm{~nm}$.

$35 \mathrm{PL}$ spectra of the $\mathrm{PbS} / \mathrm{GaAS}$ sample at various selected temperatures measured with the InGaAs detector.

$36 \mathrm{PL}$ spectra of the $\mathrm{PbS} / \mathrm{SI}$ GaAS sample at various temperatures measured with the InSb detector.

37 Comparison of the $5 \mathrm{~K} \mathrm{PL}$ in the mid-infrared range of the SI GaAs substrate and the $\mathrm{PbS} / \mathrm{SI}$ GaAs sample. The spectra are shown in the same arbitrary units.

38 Comparison of the $5 \mathrm{~K}$ PL in the near infrared range of the SI GaAs substrate and the $\mathrm{PbS} / \mathrm{GaAs}$ sample. The spectra are shown in the same arbitrary units.

39 A) PL peak position vs. temperature measured (a) with the InSb detector and (b) with the InGaAs detector. The broken and dotted lines are fitted with Equation (19). B) PL peak position vs. temperature measured (a) with the InSb detector and (b) with the InGaAs detector. The broken and dotted lines are fitted with Equation (20).

40 FWHM vs. temperature established with (a) the InSb detector and (b) the InGaAs detector. The broken and dotted lines are fitted with Equation (21).

$41 \quad$ PL peak intensity vs. temperature measured with (a) the InSb detector and

(b) InGaAs detector. The broken and dotted lines are linear fits of the data points......

$42 \mathrm{XRD}$ pattern of $\mathrm{PbS}$ nanoparticles .58

iv

Distribution Statement A. Approved for public release; distribution unlimited. 
43 TEM images of PbS QDs with different sizes. (a) size $=14.4 \pm 1.6 \mathrm{~nm}$; (b) size $=8.6 \pm 1.1 \mathrm{~nm} ;(\mathrm{c})$ size $=4.8 \pm 0.54 \mathrm{~nm} ;(d)$ size $=2.8 \pm 0.31 \mathrm{~nm}$.

44 Supercritical fluid $\mathrm{CO}_{2}$ system for deposition of nanoparticles and formation of films

45 TEM images of the nanoparticle arrays formed by bench top solvent evaporation (a) and by supercritical fluid deposition (b) of the $4.7 \mathrm{~nm} \mathrm{PbS}$ nanoparticles.

46 Sample forming apparatus for making nanoparticle films in $\mathrm{sc}-\mathrm{CO}_{2}$. (a) sc- $\mathrm{CO}_{2}$ high pressure reaction chamber, (b) an apparatus containing metal cups for $\mathrm{PbS}$ deposition, (c) $\mathrm{PbS}$ film deposited on glass using SDM and (d) sc- $\mathrm{CO}_{2}$ deposition methods. The lateral film density is about 0.49 $\mathrm{mg} / \mathrm{cm}^{2}(\mathrm{~d})$.

$47 \quad \mathrm{SEM}$ image of surface of a $\mathrm{PbS}$ nanoparticle film prepared by the sc- $\mathrm{CO}_{2}$ deposition on a $\mathrm{Cr}$ coated glass. The lateral density of $\mathrm{PbS}$ on $\mathrm{Cr}$ coated glass is around $2.2 \mathrm{mg} / \mathrm{cm}^{2}$

48 Microscope images of PbS QDs deposited on glass using solvent and sc$\mathrm{CO}_{2}$ deposition methods in the same concentration $\left(\sim 2.2 \mathrm{mg} / \mathrm{cm}^{2}\right)$. (a) solvent deposition of $\mathrm{PbS}$ (scale: $1.25 \mathrm{X}$ ), (b) sc- $\mathrm{CO}_{2}$ deposition of $\mathrm{PbS}$ (scale:1.25X), (c) solvent deposition of PbS (scale: 10X), (d) $\mathrm{sc}-\mathrm{CO}_{2}$ deposition of $\mathrm{PbS}$ (scale:10X).

49 UV-Vis-NIR measurements of $\mathrm{PbS}($ size $=2.3 \mathrm{~nm}$, top) and ( $\operatorname{size}=4.8$ $\mathrm{nm}$, bottom)

50 Absorption spectra of the $\mathrm{PbS} /$ toluene solutions in $2.7 \mathrm{~nm}$ and $4.8 \mathrm{~nm}$, measured individually (top). Absorption spectra of a mixture of $2.7 \mathrm{~nm}$ and $4.8 \mathrm{~nm} \mathrm{PbS} /$ toluene solution and the film prepared by $\mathrm{sc}-\mathrm{CO}_{2}$ evaporation method (bottom); film lateral density $\sim 2.2 \mathrm{mg} / \mathrm{cm}^{2}$.

51 Fluorescence spectra of the mixed $\mathrm{PbS}$ nanoparticles in the original toluene solution (bottom) and in the film formed by sc- $\mathrm{CO}_{2}$ deposition of the solution (top), film lateral density $\sim 2.2 \mathrm{mg} / \mathrm{cm}^{2}$.

52 PL intensity vs. wavelength and energy at $5 \mathrm{~K}$ and $300 \mathrm{~K}$ of samples formed with sc- $\mathrm{CO}_{2}$ and SDM methods

53 PL measurements of $\mathrm{PbS}$ deposited on $p$-type GaAs using the same $\mathrm{PbS}$ solution, before and after 3 months

$54 \mathrm{XRD}$ measurements of $\mathrm{PbS}$ films deposited on glass, prepared initially $(\mathrm{t}=0)$, prepared 10 months and 23 months later.

55 Homemade apparatus for solvent deposition (left) and the formed $\mathrm{PbS}$ QDs on glass (right) .72

$56 \quad \mathrm{PL}$ and $\mathrm{OA}$ spectra measured at $5 \mathrm{~K}$ .73

57 The symbols show the measured thermal shift of the PL ( $\square$ ) and OA ( $\square$ ) peaks. The dashed lines are the fits using Equation (22).

Distribution Statement A. Approved for public release; distribution unlimited. 
58 Schematic of the OA and PL transitions with hole trap states tied to the bulk $\mathrm{PbS}$ valence band edge. The shift of the trap state with temperature results in the temperature dependence of the Stokes shift.

59 The difference between the energies of the OA and PL peaks in Figure 57. The dotted line is the fit using $\left\langle E_{\mathrm{p}}\right\rangle=14.5 \mathrm{meV}, \mathrm{S}(0)=135 \mathrm{meV}$, and $C=60.2 \mathrm{meV}$ in Equation (23).

60 (a) Optical microscope image of PbS QD film on PET (10X magnification), (b) TEM image of PbS QDs on a TEM copper grid, both samples fabricated using CDM.

$61 \quad$ PL spectra at $5 \mathrm{~K}$ and $300 \mathrm{~K}$. The broken line was measured and the solid lines represent the fits using Equation (25)...

62 Peak energy position vs. temperature evaluated with the Gaussian fits using Equation 25.

63 PL peak intensity (parameter $A$ in Equation 25) vs. temperature evaluated from the Gaussian fits.

64 FWHM dependence of the PL spectra vs. temperature: The symbols along curve (a) represent the FWHM determined from the PL fits using Equation (25). The guide for the eyes, the dotted line, is a polynomial fit. The broken line (b) shows the calculated expected behavior based on the Bose distribution function of longitudinal optical phonons [13,22].

65 Optical microscope images of (top) $\mathrm{PbS}$ (SDM) on S.I. GaAs substrate and (bottom) $\mathrm{PbS}$ (SFD) on S.I.GaAs substrate.

66 (top) Absorbance spectra of PbS QDs on glass using SFD. The duration between 2 measurements is 4.3 months; (bottom) Absorbance spectra of $\mathrm{PbS}$ QDs on glass using SDM. The duration between 2 measurement is around 4.6 months.

67 Room temperature photoluminescence measurements over three months on samples a) SDM and b) SFD deposited on glass.

68 Room temperature photoluminescence measurements over three months on samples a) SDM and b) SFD deposited on GaAs.

$69 \mathrm{~Pb} 4 \mathrm{f}$ and $\mathrm{S} 2 \mathrm{~s}$ photoelectron transitions obtained from the SFD and SDM of PbS QD films; with chemical states fitted.

70 XRD measurements of a PbS film (4.7 nm) measured in 2009 (top) and 2012 (bottom) which were prepared by SDM.

71 (a) $10 \mu \mathrm{m} \times 10 \mu \mathrm{m}$ image of the general surface morphology; (b) 3D representation of Figure 71a

$72 \mathrm{SDM}(\mathrm{a}, \mathrm{b}, \mathrm{c})$ and $\mathrm{scCO}_{2}(\mathrm{~d}, \mathrm{e}, \mathrm{f})$ deposition of $\mathrm{PbS}$ on carbon coated cupper grids, which were stored under ambient conditions about 2 years and 3 months. TEM images $(\mathrm{a}, \mathrm{d})$ were taken on Day 1 , TEM images $(\mathrm{b}, \mathrm{e})$ were taken on Day 56, and TEM images (c, f) were taken on Day 967.

vi

Distribution Statement A. Approved for public release; distribution unlimited. 


\section{LIST OF TABLES}

Table

1 Experimental and theoretical results for the InAs/GaSb SLs used in the transport study. Mobilities and sheet carrier concentrations are $10 \mathrm{~K}$ values. Since each SL has 40 periods, the sheet carrier densities per period are found by dividing the fourth column by 40 . The band gaps were calculated using the modified 8x8 EFA model with InSb-like interfaces

2 Results of fitting the experimental mobilities in Table 1. The Fermi wave vector was found from Equation (14); the prefactor $C\left(\Delta, \Lambda, k_{F}\right)$ is found from Equation (13) and the experimental mobilities in Table 1. The correlation lengths are found using Equation (15) assuming a one monolayer roughness, $\Delta=3.0 \AA$

3 Summary of the results obtained from multi-carrier analysis of the data from $21 \AA(\mathrm{InAs}+\mathrm{IF}) / 24 \AA(\mathrm{GaSb}+\mathrm{IF})$ superlattices. The $0.3 \AA$ of InSblike or/and GaAs-like interfaces (IF) were inserted between the layers and their values were estimated from shutter time. The $0 / 0$ represents uncontrolled IF. The Rs, ns and $\mu$ represent the 10-K resistivity, hole density and in-plane hole mobility.

$4 \quad$ Hall and photoluminescence (PL) data for the InAs/GaSb superlattices. The InAs width was fixed at $21 \AA$. All SLs in the series were p-type.

Three parameters $\mathrm{R}, \mathrm{n}$, and $\mu$ represent the resistivity, hole concentration, and mobility respectively

$5 \quad$ Fitting parameters used with the Varshni relation ......................................................52

$6 \quad$ Fitting parameters used with the O'Donnell-Chen relation................................................52

$7 \quad$ Fitting parameters used with the Bose phonon distribution function ................................53

$8 \quad$ Fitting parameters for the fits in Figure 57 done with Equation (22) ................................74

9 Summary of Gaussian fit parameters to the PL spectra ...................................................89

Distribution Statement A. Approved for public release; distribution unlimited. 


\subsection{INTRODUCTION}

This research task had an overall objective of pushing forward the state-of-the-art in utilizing semiconductor quantum confined materials such as superlattices and quantum dots in optical and optoelectronic devices of interest to the Air Force. The specific materials studied were InAs/GaSb superlattices, InGaAs epitaxial quantum dots in a GaAs matrix and $\mathrm{PbS}$ colloidal quantum dots. The deposition processes used were molecular beam epitaxy and supercritical fluid $\mathrm{CO}_{2}$ deposition. The physical properties of these quantum confined materials were studied using a variety of structural, optical and electrical characterization techniques available in-house. There were 37 journal articles and 20 proceedings papers written on the research results. The complete listing is at the end of the report.

To capture some of the results accomplished and reported under this research task selected published papers are incorporated into the report. The papers are broken into two sections: I. Superlattice Research and II. Quantum Dot Research. These selected papers demonstrate the breadth of the effort and highlight different aspects of the research effort. Further details can be explored by reading other papers by the team included in the publication list.

Distribution Statement A. Approved for public release; distribution unlimited. Approved by 88ABW Public Affairs Office: Case number 88ABW-2015-1223 on 19-MAR-2015. 


\subsection{SUPERLATTICE RESEARCH PAPERS}

2.1 Interface roughness estimate from carrier transport in InAs/GaSb superlattices F. Szmulowicz, University of Dayton Research Institute, Dayton, Ohio 45469-0178, USA

S. Elhamri, Department of Physics, University of Dayton, Dayton, Ohio 45469-2314, USA

H. J. Haugan, Universal Technology Corporation, Dayton, Ohio 45432, USA

G. J. Brown and W. C. Mitchel, Air Force Research Laboratory, Materials \& Manufacturing Directorate, Wright-Patterson Air Force Base, Ohio 45433, USA

The performance of infrared focal plane arrays and quantum cascade lasers manufactured from InAs/GaSb type-II superlattices (SLs) depends on the mobility of carriers along the growth axis. In turn, the longitudinal mobility depends on the quality of SL interfaces. In-plane transport is a sensitive measure of interface quality and the degree of interface roughness scattering (IRS). In this paper, we demonstrate the IRS-limited transport regime in InAs/GaSb SL samples grown for this study. We find that the in-plane mobility $\mu$ as a function of InAs layer width $L$ behaves as $\mu \propto L^{5}$, which closely follows the classic sixth power dependence expected from theory. Fits to the mobility data indicate that, for one monolayer surface roughness, the roughness correlation length is about $35 \AA$.

Keywords: InAs/GaSb, superlattice, mobility, transport, interface roughness scattering

\section{INTRODUCTION}

InAs/GaSb superlattices (SLs) are promising candidate materials for third generation IR focal plane arrays. ${ }^{1-10}$ the performance of these arrays depends on the optical and transport properties of the superlattice. The vertical transport of carriers in SLs is affected by intrinsic scattering mechanisms as well as by such extrinsic mechanisms as interface roughness scattering (IRS). ${ }^{11-13}$ Interface roughness is characterized by statistical fluctuations in layer width and their lateral extent given by the correlation length. ${ }^{14-17}$ Vertical transport has been studied by several authors, who found that interface roughness dominates longitudinal transport from cryogenic to the room temperature and that mobilities depend sensitively on the SL period. ${ }^{12,13}$

Here, we study interface roughness by examining the low temperature in-plane transport in SLs since at cryogenic temperatures phonon scattering and substrate conduction are frozen out. Theoretical treatments ascribe IRS to layer thickness fluctuations that cause local variations in electronic energy levels. ${ }^{18-20}$ For samples dominated by the IRS, theory predicts that the in-plane mobility will depend on the sixth-power of layer width ${ }^{18,19}$ Using two parameters - height, $\Delta$ and a correlation length, $\Lambda$ of Gaussian-like fluctuations - Gold $^{19}$ derived the following relation for IRS mobility

$$
\mu=\frac{e L^{6}}{\pi^{5} \hbar \Delta^{2} \Lambda^{2}} \times F\left(\Lambda, k_{F}\right)^{-1},
$$

where $L$ is the width of the current-carrying layer and $F\left(\Lambda, k_{F}\right)$ is a scattering factor that depends on the Fermi wave vector $k_{F}$ and screening. As long as the carrier wave function does not appreciably penetrate into the adjoining GaSb layers, Eq. (1) gives rise to the classic $L^{6}$ dependence of mobility on layer width. ${ }^{18-20}$ Refinements of the theory were proposed by Nag et $a l^{21}$, Li et ll $^{22}$, and Nag. ${ }^{23}$ Earlier experimental studies in InAs/ $\mathrm{Ga}_{0.75} \mathrm{In}_{0.25} \mathrm{Sb}$ SLs found a

Distribution Statement A. Approved for public release; distribution unlimited. 
weaker power dependence ${ }^{17}$ that was attributed to carrier penetration into and parallel conduction in the InGaSb barrier region.

In what follows, Section 2 lists the experimental details about the growth and measurement. Section 3 describes and discusses the results and Section 4 gives a detailed account of the theory and data fitting in order to extract the interface roughness parameters from the data. Conclusions are presented last.

\section{EXPERIMENTAL DETAILS}

In previous papers, we reported on the growth of binary-binary InAs/GaSb superlattices. ${ }^{24,25}$ By demonstrating IRS limited mobilities in these samples, the present Hall effect measurements are used to confirm our earlier assessments of their high quality. ${ }^{24,25}$ The SLs were grown on GaSb substrates that were unintentionally p-type. The substrate temperature was set at $410 \pm 10{ }^{\circ} \mathrm{C}$, and the V/III beam flux ratio was about 3-4 for GaSb and InAs. The GaSb layer width was fixed at $24 \AA$ while the InAs layer width varied from 30 to $70 \AA$ in $10 \AA$ steps and each sample had forty periods. The periods were inferred from high resolution X-ray rocking curves while the layer widths were based on calibrated growth rates. The interface type was not controlled. While the superlattices were not intentionally doped, the four widest period SLs were residually n-type; the $30 \AA \mathrm{SL}$ was p-type.

Transport measurements were performed on $1 \mathrm{~cm} \times 1 \mathrm{~cm}$ van der Pauw samples. Variable temperature $(10-300 \mathrm{~K})$ resistivity and single field $(0.5 \mathrm{~T})$ Hall effect measurements were carried out in a guarded direct-current system. In order to eliminate parallel conduction through the substrate, the study of the mobility as a function of the InAs thickness was conducted below 20 $\mathrm{K}$ since the substrate was shown to freeze out below this temperature.

\section{RESULTS AND DISCUSSION}

The results of our study on a set of five samples are listed in Table 1. We also calculated the electronic structure for the five samples, using the $8 \times 8$ envelope function approximation model. ${ }^{26,27}$ Theory predicts all the samples to be semiconducting.

First, we measured the temperature dependence of the resistivity and the Hall effect between 10 and $300 \mathrm{~K}$. Based on these measurements, we ascertained that the p-type conduction channel in the substrate is negligible below $20 \mathrm{~K}$. The elimination of the substrate's contribution to conduction makes it possible for us to study the effect of InAs width on carrier mobility. The variable temperature Hall measurements indicated that, unlike the first superlattice, the last four samples undergo a type conversion, from $\mathrm{p}$ to $\mathrm{n}$-type, and are all $\mathrm{n}$-type below $20 \mathrm{~K} .^{28}$

Distribution Statement A. Approved for public release; distribution unlimited. 
Table 1. Experimental and theoretical results for the InAs/GaSb SLs used in the transport study. Mobilities and sheet carrier concentrations are $10 \mathrm{~K}$ values. Since each SL has 40 periods, the sheet carrier densities per period are found by dividing the fourth column by

40. The band gaps were calculated using the modified 8x8 EFA model with InSb-like interfaces.

\begin{tabular}{|c|c|c|c|c|c|}
\hline Sample & $\begin{array}{c}\text { InAs } \\
\text { Width }(\AA)\end{array}$ & $\begin{array}{c}\text { GaSb } \\
\text { Width }(\AA)\end{array}$ & $\begin{array}{c}\text { Sheet Carrier } \\
\text { Concentration } \\
\left.\mathbf{( x 1 0}^{\mathbf{1 2}} \mathbf{c m}^{-2}\right)\end{array}$ & $\begin{array}{c}\text { Measured } \\
\text { Mobilities } \\
\mathbf{c m}^{\mathbf{2}} / \mathbf{V} \text {-sec) }\end{array}$ & $\begin{array}{c}\text { Calculated } \\
\text { Band Gaps } \\
(\mathbf{m e V})\end{array}$ \\
\hline SL1 & 30.5 & 24.0 & 9.0 p-type & 79 & 194.4 \\
SL2 & 41.1 & 24.0 & 0.9 n-type & 747 & 120.2 \\
SL3 & 53.6 & 24.0 & 1.2 n-type & 5,043 & 66.6 \\
SL4 & 62.3 & 24.0 & 1.6 n-type & 8,980 & 47.3 \\
SL5 & 72.7 & 24.0 & 1.5 n-type & 15,450 & 43.0 \\
\hline
\end{tabular}

Figure 1 summarizes the measured mobilities as a function of temperature, in the range where the substrate is frozen out, for all five samples in the study. The mobilities are temperature independent, which rules out intrinsic phonon scattering mechanisms (acoustic, piezoelectric, polar and non-polar optical) and the ionized impurity scattering. Since InAs and GaSb are binary compounds, alloy scattering is inoperative in our samples.

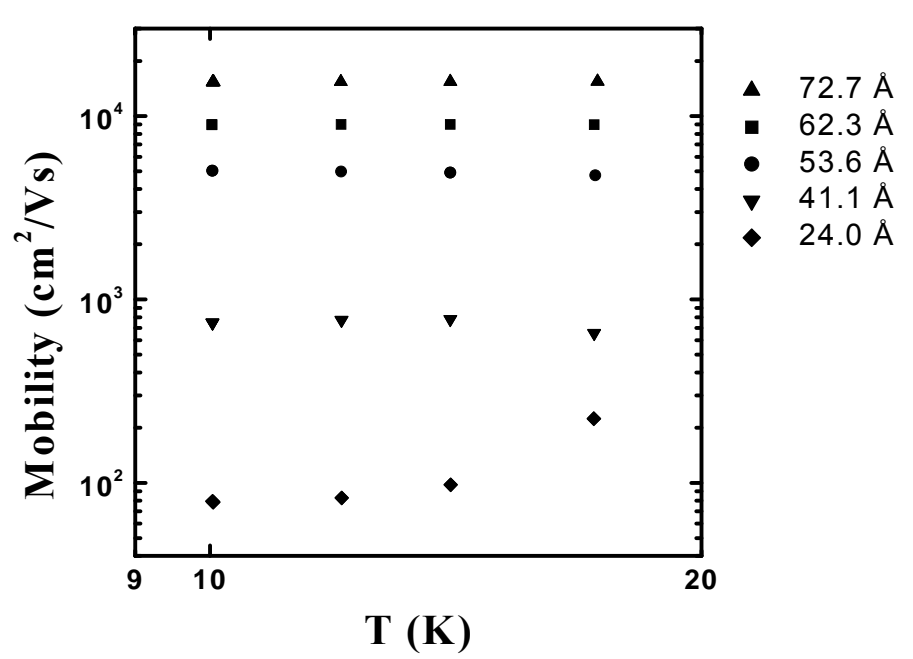

Figure 1. Mobilities as a function of temperature in the temperature regime below the type conversion. ( $\triangle$ : SL5, $\square:$ SL4, $\bullet:$ SL3, ○:SL2, n:SL1)

The inclusion of SL1 in the study merits explanation. Unlike the other four samples, SL1 is ptype, so that its mobility refers to holes confined in the GaSb layer. However, the EFA calculation shows that the in-plane effective mass of electrons in the first conduction band, $m_{C}=$ 0.023 , and of heavy holes in the first valence band, $m_{H}=0.038$, are on the same order of magnitude. Also, the Fermi wave vector in two dimensions is independent of mass (Equation 13 in the text). Lastly, IRS-limited mobilities in Gold model ${ }^{19}$ are also independent of mass (Equation 11 in the text). In fact, the conditions of the theory requiring negligible penetration of the wave function into the adjoining barrier regions are better satisfied for holes because of their much heavier mass in the growth direction.

Distribution Statement A. Approved for public release; distribution unlimited. 
The $10 \mathrm{~K}$ mobilities as a function of InAs layer width (for SL2 - SL5) and GaSb width (for SL1) are plotted in Figure 2. Several polynomial fits to the data were attempted:
a) fitting the data for SL2 through SL5 gave

$$
\mu=\left(2.17 \times 10^{-6}\right) L^{5.3} \quad \text { (goodness of fit } \mathrm{R}=0.982 \text { ); }
$$
b) fitting the data for SL1 through SL5 gave

$$
\mu=\left(1.10 \times 10^{-5}\right) L^{4.9} \quad \text { (goodness of fit } \mathrm{R}=0.988 \text { ), }
$$

where the mobility is in $\mathrm{cm}^{2} / \mathrm{V}$-s and the InAs layer width $L$ is in angstroms. We infer then that, to within experimental accuracy, the mobilities behave as the $L^{5}$ power of layer width. Since this closely follows the $\mu \propto L^{6}$ dependence predicted by theory, ${ }^{18}$ we conclude that IRS is the dominant low-temperature scattering mechanisms in our samples, which requires that layer width variations be on the order of a monolayer ( $3 \AA$ ). The remaining discrepancy between experiment and the model can be ascribed to experimental factors such as interface interdiffusion and theoretical factors such as wave function penetration into barrier regions.

The only comparable study of the present system is the study of $\mathrm{InAs} / \mathrm{In}_{0.25} \mathrm{Ga}_{0.75} \mathrm{Sb}$ SLs by Hoffman et al. ${ }^{17}$ who found a much lower power exponent dependence, $\mu \propto L^{2.4}$. These authors attribute this behavior to wave function penetration into the barrier region. We also note the following differences in our samples: Hoffman et al. use the ternary barrier layers which can admix alloy scattering; they intentionally dope their SLs, whereas ours are nominally undoped; which reduces impurity scattering in our samples; and they use GaAs substrates, whereas our $\mathrm{SLs}$ are grown on closer lattice matched GaSb substrates.

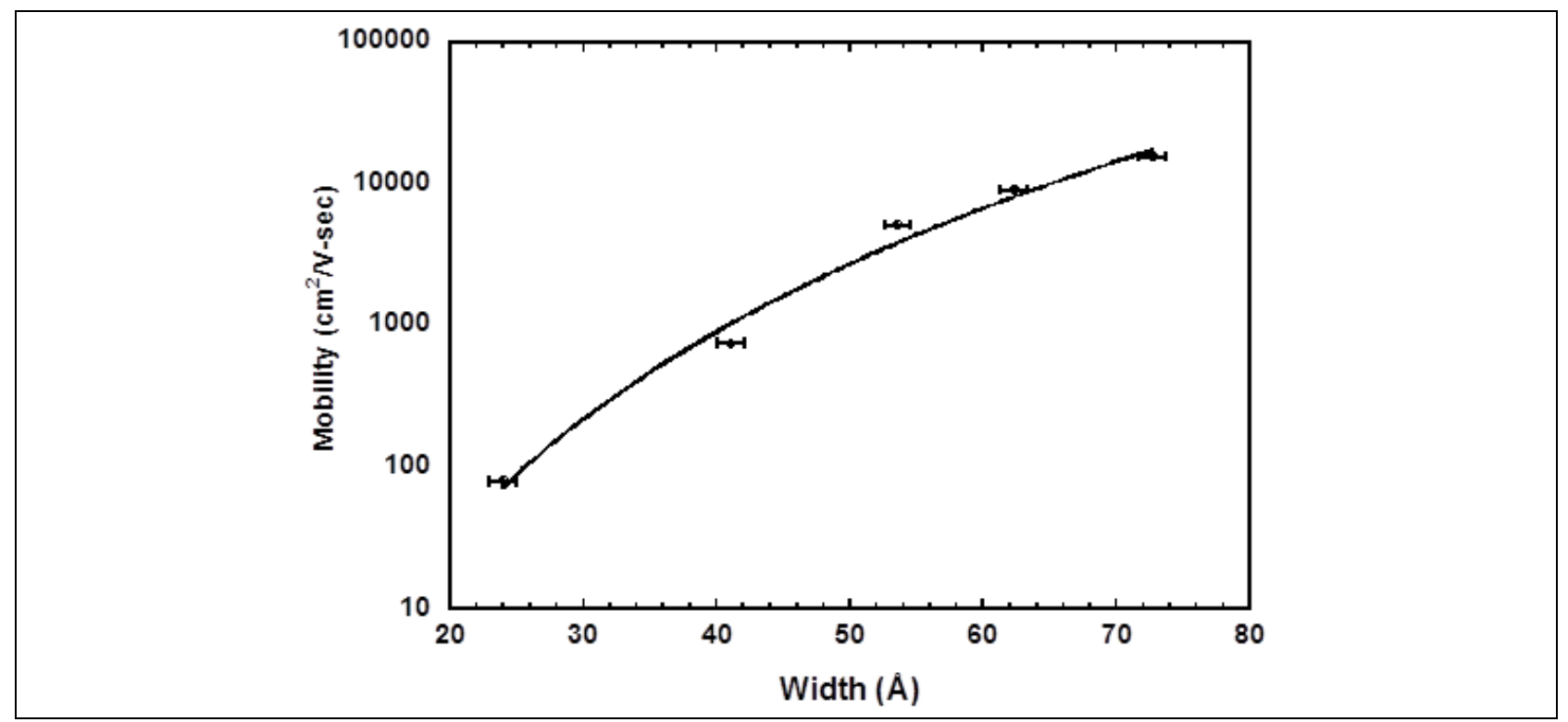

Figure 2. The layer width dependence of the measured mobilities (filled circles) together with the best power law fit (solid curve).

Distribution Statement A. Approved for public release; distribution unlimited. 


\section{ROUGHNESS ANALYSIS}

In this section, we explain the fitting procedure used to extract interface roughness and correlation by fitting our mobility data to the available models. ${ }^{18-23}$ Mobility $\mu$ is proportional to the average relaxation time $\left\langle\tau_{R}\right\rangle$,

$$
\mu=\frac{e}{m^{*}}\left\langle\tau_{R}\right\rangle
$$

where $m^{*}$ is the carrier in-plane effective mass. In order to determine the average relaxation time, the energy dependent relaxation time $\tau_{R}(E)$ is weighted by the Fermi-Dirac distribution $f_{0}(E)$ as

$$
\left\langle\tau_{R}\right\rangle=\frac{\int \tau_{R}(E) E \frac{\partial f_{0}(E)}{\partial E} d E}{\int E \frac{\partial f_{0}(E)}{\partial E} d E}
$$

In turn, the energy dependent relaxation time is found from

$$
\frac{1}{\tau_{R}(E)}=\frac{1}{(2 \pi)^{2}}\left(\frac{2 \pi}{\hbar}\right) \int S_{C}(q) M_{R}^{2}(1-\cos \theta) \delta\left(E_{\mathbf{k}^{\prime}}-E_{\mathbf{k}}\right) d^{2} \mathbf{k}^{\prime}
$$

which considers all elastic scattering events on the energy shell $E=E_{\mathbf{k}^{\prime}}=E_{\mathbf{k}}$, which is appropriate for all scattering mechanisms other than the polar and nonpolar optical phonon scattering mechanisms, which are inelastic. The strength of interface scattering in Gold's model is given by $^{19}$

$$
M_{R}^{2}=\frac{\pi^{5} \hbar^{4} \Delta^{2} \Lambda^{2}}{m^{*^{2}} L^{6}} \exp \left(-\frac{\Lambda^{2} q^{2}}{4}\right),
$$

where $\mathbf{q}=\mathbf{k}-\mathbf{k}^{\prime}$ is the momentum transfer, $\Delta$ is the interface roughness, $\Lambda$ is the coherence length of interface fluctuations, and $L$ is the width of the current-carrying layer. Screening is accounted for by the term

$$
S_{C}(q)=\left(\frac{q}{q+q_{s}}\right)^{2},
$$

where $q_{s}$ is the inverse screening length given by

$$
q_{s}=\frac{m^{\bullet}(E) e^{2}}{2 \pi \varepsilon \hbar^{2}}
$$

Distribution Statement A. Approved for public release; distribution unlimited. 
where $\varepsilon$ is the static dielectric constant. For the numbers appropriate to InAs, the screening length is estimated to be about $q_{s}^{-1} \approx 180 \AA$. For the carrier densities in Table $1, k_{F}^{-1} \approx 200 \AA$, which is on the order of $q_{s}^{-1}$; therefore, electronic screening cannot be neglected.

Next, integrating the expression in Equations (5 ) and (6), J M Li showed that ${ }^{22}$,

$$
\frac{1}{\tau_{R}(E)}=\frac{\pi^{5} \hbar \Delta^{2} \Lambda^{2}}{m^{*} L^{6}} F\left(\Lambda, k_{F}\right)
$$

where the scattering integral

$$
F(\Lambda, k)=\frac{1}{2 \pi} \int S_{C} \exp \left(-\frac{\Lambda^{2} k^{2}(1-\cos \theta)}{2}\right)(1-\cos \theta) d \theta,
$$

and $k_{F}$ is the Fermi wave vector. Altogether, the expression for the IRS mobility is given by

$$
\mu=\frac{e L^{6}}{\pi^{5} \hbar \Delta^{2} \Lambda^{2}} \times F\left(\Lambda, k_{F}\right)^{-1}
$$

Letting $\ell, \lambda$, and $\delta$ be the numerical values of $L, \Lambda$, and $\Delta$ in $\AA$, Equation (11) can be rewritten as

$$
\mu=4.9644 \times 10^{-4} \frac{\mathrm{cm}^{2}}{V-\sec }\left(\frac{1}{F\left(\Lambda, k_{F}\right)(\delta \lambda)^{2}}\right) \ell^{6} .
$$

In order to extract the values of the interface roughness parameters, it is necessary to force the $L^{6}$ dependence from the model, Equation (11) by finding the experimental values of the constant $C\left(\Delta, \Lambda, k_{F}\right)$, using the expression

$$
\mu=C\left(\Delta, \Lambda, k_{F}\right) L^{6}
$$

From Equation (13), the experimental values of the constant $C\left(\Delta, \Lambda, k_{F}\right)$ are listed for all the samples in the study in Table 2. The constant is a function of IRS parameters and the Fermi wave vector. The Fermi wave vector is found from the carrier sheet density

$$
k_{F}^{2}=2 \pi n_{\text {Sheet }},
$$

and is also tabulated in Table 2. Overall, the experimental-theoretical fit entails finding the values of $\lambda$ and $\delta$ that satisfy the equation

$$
4.9644 \times 10^{-4} \frac{\mathrm{cm}^{2}}{V-\sec }\left(\frac{1}{F\left(\Lambda, k_{F}\right)(\delta \lambda)^{2}}\right)=C\left(\Delta, \Lambda, k_{F}\right) .
$$

Distribution Statement A. Approved for public release; distribution unlimited. 
The scattering integral $F\left(\Lambda, k_{F}\right)$ is calculated from Equation (10). In the limit of vanishingly small sheet carrier density, the integral $F\left(\Lambda, k_{F}\right) \rightarrow 1$. In general, the integral must be calculated numerically as a function of $\Lambda k_{F}$. The scattering factor $F\left(\Lambda, k_{F}\right)$ as a function of the product $\Lambda k_{F}$ for $S_{C}=1$ is shown in Figure 3 .

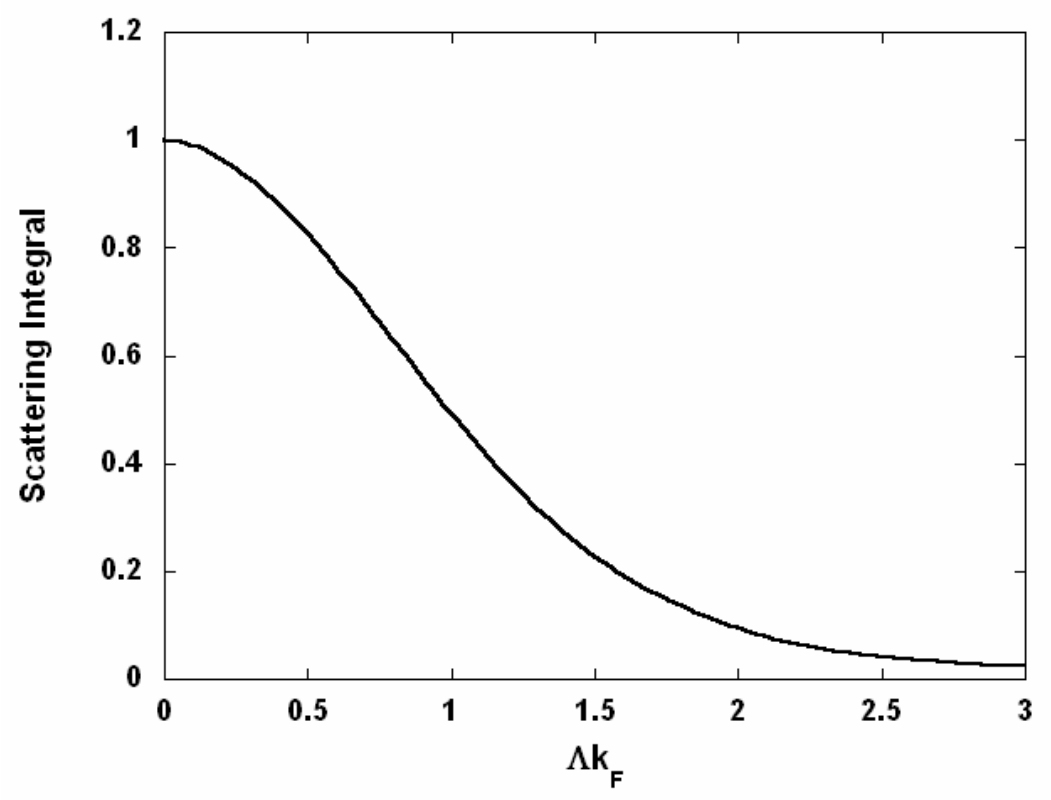

Figure 3. The scattering integral (dimensionless) $F\left(\Lambda, k_{F}\right)$, Equation (10), as a function of the product $\Lambda k_{F}$ (dimensionless), where $\Lambda$ is the roughness correlation length and $k_{F}$ is the Fermi wave vector. Here, the screening factor $S_{C}=1$.

However, for the samples listed in Table 2, the average Fermi wave vector is about $0.0045 \AA^{-1}$. If the correlation lengths are on the order of $30 \AA$, then $\Lambda k_{F}$ is only 0.15 . From Figure 3 , this is in the small $\Lambda k_{F}$ regime so that the scattering integral can be well approximated by its low order expansion

$$
F\left(\Lambda, k_{F}\right)=1-3 \Lambda^{2} k_{F}^{2}+\cdots .
$$

If one uses just $F\left(\Lambda, k_{F}\right)=1$ and assumes a one-monolayer roughness, $\delta=3.0$, the correlations lengths are found to be on the order of $35 \AA$, Table 2, so using the first term in Equation (15) is accurate to within $2 \%$.

These numbers compare favorably with direct measurements of interface roughness using scanning tunneling microscopy. For example, Feenstra et al. ${ }^{29}$ found different degrees of IRS for InAs on $\mathrm{GaSb}(\Delta=1.8 \AA, \Lambda=19 \AA)$ and GaSb on InAs interfaces $(\Delta=2.5 \AA, \Lambda=40 \AA)$. However, longer correlation lengths are found by Lew et al. ${ }^{30}$ who find $(\Delta=1.9 \pm 0.1 \AA, \Lambda=112 \pm 16 \AA$; $\Delta=2.8 \pm 0.2 \AA, \Lambda=174 \pm 21 \AA$ ) for InAs on InGaSb and $(\Delta=3.2 \pm 0.2 \AA, \Lambda=301 \pm 39 \AA ; \Delta=4.3 \pm 0.2 \AA$, $\Lambda=327 \pm 38 \AA$ ) for InGaSb on InAs interfaces in (110)/(1-10) cross-sections.

Distribution Statement A. Approved for public release; distribution unlimited. 
Table 2. Results of fitting the experimental mobilities in Table 1. The Fermi wave vector was found from Equation (14); the prefactor $C\left(\Delta, \Lambda, k_{F}\right)$ is found from Equation (13) and the experimental mobilities in Table 1. The correlation lengths are found using Equation (15) assuming a one monolayer roughness, $\Delta=3.0 \AA$.

\begin{tabular}{|c|c|c|c|c|}
\hline Sample & $k_{F}\left(\AA^{-1}\right)$ & $\begin{array}{c}C\left(\Delta, \Lambda, k_{F}\right) \\
\frac{c m^{2} \AA^{-6}}{V-\sec }\end{array}$ & Screening Factor $S_{C}$ & $\Lambda(\AA)$ for $\Delta=3 \AA$ \\
\hline SL1 & 0.012 & $1.05 \times 10^{-7}$ & 0.466 & 33.6 \\
SL2 & 0.0038 & $1.54 \times 10^{-7}$ & 0.164 & 46.7 \\
SL3 & 0.0043 & $2.13 \times 10^{-7}$ & 0.189 & 37.0 \\
SL4 & 0.0050 & $1.55 \times 10^{-7}$ & 0.223 & 39.9 \\
SL5 & 0.0049 & $4.13 \times 10^{-7}$ & 0.219 & 24.7 \\
\hline
\end{tabular}

\section{CONCLUSIONS}

We performed a designed study of transport in InAs/GaSb type-II superlattices. The in-plane carrier transport in SLs was examined as a function of layer width $L$. We found that the lowtemperature mobilities followed closely the $\mu \propto L^{5}$ dependence expected for the interfacescattering limited mobility regime. From our fits of the mobility data to the model of Gold, and assuming a one-monolayer roughness $\Delta=3.0 \AA$, we infer that the roughness in our SLs is characterized by the correlation length of about $\Lambda=35 \AA$. The absence of defect-related scattering is indicative of good quality of the present set of InAs/GaSb SLs.

\section{REFERENCES}

1. A. Rogalski and P. Martyniuk, "InAs/GaInSb superlattices as a promising material system for third generation infrared detectors," Infrared Physics \& Technology 48, 39-52 (2006).

2. Q.K.Yang, F. Fuchs, J. Schmitz, and W. Pletschen, "Investigation of trap-assisted tunneling current in InAs/(GaIn)Sb superlattice long-wavelength photodiodes," Appl. Phys. Lett. 81, 4757-4759 (2002).

3. R. Rehm, M. Walther, F. Fuchs, J. Schmitz, and J.H. Fleissner, "Passivation of $\mathrm{InAs} /(\mathrm{GaIn}) \mathrm{Sb}$ short-period superlattice photodiodes with $10 \mu \mathrm{m}$ cutoff wavelength by epitaxial overgrowth with $\mathrm{Al}_{x} \mathrm{Ga}_{1-x} \mathrm{As}_{y} \mathrm{Sb}_{1-y}$," Appl. Phys. Lett. 86, 173501 (2005).

4. A. Gin, Y. Wei, A. Hood, A. Bajowala, V. Yazdanpanah, M. Razeghi, and M. Z. Tidrow, "Ammonium sulfide passivation of Type-II InAs/GaSb superlattice photodiodes," Appl. Phys. Lett. 84, 2037-2039 (2004).

5. A. Hood, M. Razeghi, E.H. Aifer, and G.J. Brown, "On the performance and surface passivation of type II InAs/GaSb superlattice photodiodes for the very-long-wavelength infrared," Appl. Phys. Lett. 87, 151113 (2005); A. Hood, D. Hoffman, B.-M. Nguyen, P.Y. Delaunay, E. Michel, and M. Razeghi, "High differential resistance type-II InAs/GaSb superlattice photodiodes for the long-wavelength infrared," Appl. Phys. Lett. 89, 093506 (2006).

6. E.H. Eifer, J.G. Tischler, J.H. Warner, I. Vurgaftman, W.W. Bewley, J. R. Meyer, J.C. Kim, L.J. Whitman, C.I. Canedy, and E.M. Jackson, "W-structured type-II superlattice long-wave infrared photodiodes with high quantum efficiency," Appl. Phys. Lett. 89, 053519 (2006).

Distribution Statement A. Approved for public release; distribution unlimited. 
7. F. Fuchs, L. Burkle, R. Hamid, N. Herres, W. Platschen, R.E. Sah, R. Keifer, and J. Schmitz, "Optoelectronic properties of photodiodes for the mid-and far-infrared based on the InAs/GaSb/AlSb materials family," Proc. SPIE 4288, 171-182 (2001).

8. I. Vurgaftman, E.H. Aifer, C.L. Canedy, J.H. Tischler, J.R. Meyer, H.H. Warner, R.M. Jackson, G. Hildebrandt, and G.J. Sullivan, "Graded band gap for dark-current suppression in long-wave infrared W-structured type-II superlattice photodiodes," Appl. Phys. Lett. 89, 121114 (2006).

9. H.J. Haugan, F. Szmulowicz, K. Mahalingam, G.J. Brown, S.R Munshi, and B. Ullrich, "Short-period InAs/GaSb type-II superlattices for mid-infrared detectors," Appl. Phys. Lett. 87, 261106 (2005).

10. F. Szmulowicz, H.J. Haugan, G.J. Brown, K. Mahalingam, B. Ullrich, S.R Munshi, and L. Grazulis, "Interfaces as design tools for short-period InAs/GaSb type-II superlattices for mid-infrared detectors," Opto-Electronics Rev. 14, 69-75 (2006).

11. F. Aristone, P. Gassot, J.F. Palmier, D.K. Maude, B. Goutiers, J.L. Gauffier, J. C. Portal, and F. Mollot, "Probing the interface fluctuations in semiconductor superlattices using a magneto-transport technique," Superlattices and Microstructures 15, 225-228 (1994).

12. I. Dharssi and P.N. Butcher, "Interface roughness scattering in a superlattice," J. Phys. Condens. Phys. 2, 4629-4635 (1990).

13. G. Etamadi and J.F. Palmier, "Effect of interface roughness on non-linear vertical transport in GaAs/AlAs superlattices," Solid State Commun. 86, 739-743 (1993).

14. H. Sakaki, T. Noda, K. Hirakawa, M. Tanaka, and T. Matsusue, "Interface roughness scattering in GaAs/AlAs quantum wells," Appl. Phys. Lett. 51, 1934-1936 (1987).

15. R. Gottinger, A. Gold, G. Abstreiter, G. Weimann, and W. Schlapp, "Interface Roughness Scattering and Electron Mobilities in Thin GaAs Quantum Wells," Europhys. Lett. 6, 183188 (1988).

16. J.R. Meyer, D.J. Arnold, C.A. Hoffman, F.H. Bartoli, and L.R. Ram-Mohan, "Electron and hole in-plane mobilities in HgTe-CdTe superlattices," Phys. Rev. B 46, 4139-4146 (1992).

17. C.A. Hoffman, J.R. Meyer, E.R. Youngdale, F.J. Bartoli, and H.R. Miles, "Interface roughness scattering in semiconducting and semimetallic InAs- $\mathrm{Ga}_{1-x} \mathrm{In}_{x} \mathrm{Sb}$ superlattices," Appl. Phys. Lett. 63, 2210 (1993).

18. H. Sakaki, T. Noda, K. Hirakawa, M. Tanaka, and T. Matsusue, "Interface roughness scattering in GaAs/AlAs quantum wells," Appl. Phys. Lett. 41 1934-1936 (1987).

19. A. Gold, "Electronic transport properties of a two-dimensional electron gas in a silicon quantum-well structure at low temperature," Phys. Rev. B 35, 723-733 (1987).

20. R.W. Prange and T.W. Nee, "Quantum Spectroscopy of the Low-Field Oscillations in the Surface Impedance,” Phys. Rev. 168, 779-786 (1986).

21. B.R. Nag, S. Mukyopadhyay, and M. Das, "Interface roughness scattering-limited electron mobility in AlAs/GaAs and $\mathrm{Ga}_{0.5} \mathrm{In}_{0.5} \mathrm{P} / \mathrm{GaAs}$ wells," J. Appl. Phys. 86, 459-463 (1999).

22. J.M. Li, J.J. Wu, X.X. Han, Y.W. Lu, X.L. Liu, Q.S. Zhu, and Z.G. Wang, “A model for scattering due to interface roughness in finite quantum wells," Semicond. Sci. Technol. 20, 1207-1212 (2005).

Distribution Statement A. Approved for public release; distribution unlimited. 
23. B.R. Nag, "Interface roughness scattering limited mobility in AlAs/GaAs, $\mathrm{Al}_{0.3} \mathrm{Ga}_{0.7} \mathrm{As} / \mathrm{GaAs}$ and $\mathrm{Ga}_{0.5} \mathrm{In}_{0.5} \mathrm{P} / \mathrm{GaAs}$ quantum wells, " Semicond. Sci. Technol. 19, 162166 (2004).

24. H.J. Haugan, L. Grazulis, G.J. Brown, K. Mahalingam, and D.H. Tomich, "Exploring optimum growth for high quality InAs/GaSb type-II superlattices," J. Cryst. Growth 261, 471-478 (2004).

25. H.J. Haugan, F. Szmulowicz, K. Mahalingam, G.J. Brown, S.R. Munshi, and B. Ullrich, "Short-period InAs/GaSb type-II superlattices for mid-infrared detectors," Appl. Phys. Lett. 87, 261106 (2005).

26. F. Szmulowicz, H. Haugan, and G.J. Brown, "Effect of interfaces and the spin-orbit band on the band gaps of InAs/GaSb superlattices beyond the standard envelope-function approximation," Phys. Rev. B 69, 155321 (2004).

27. H.J. Haugan, F. Szmulowicz, G.J. Brown, and K. Mahalingam, "Optimization of midinfrared InAs/GaSb type-II superlattices.” Appl. Phys. Lett. 84, 5410-5412 (2004); H. J. Haugan, F. Szmulowicz, G. J. Brown, and K. Mahalingam, "Band gap tuning of InAs/GaSb type-II superlattices for mid-infrared detection,” J. Appl. Phys. 96, 2580-2585 (2004).

28. F. Szmulowicz' S. Elhamri, H.J. Haugan' G.J. Brown, and W.C. Mitchel, "Demonstration of interface-scattering-limited electron mobilities in InAs/GaSb superlattices," J. Appl. Phys. 101, 043706 (2007).

29. R.M. Feenstra, D.A. Collins, D. Z.-T. Ting, M.W. Wang, and T.C. McGill, "Interface roughness and asymmetry in InAs/GaSb superlattices studied by scanning tunneling microscopy," Phys. Rev. Lett. 72, 2749-2752 (1994).

30. A.Y. Yew, S.L. Zuo, E.T. Yu, and R.H. Miles, "Anisotropy and growth-sequence dependence of atomic-scale interface structure in InAs/Ga ${ }_{1-x} \operatorname{In}_{x} S b$ superlattices," Appl. Phys. Lett. 70, 75-77 (1997); "Correlation between atomic-scale structure and mobility anisotropy in InAs/Ga I-x $_{x} \mathrm{In}_{x} \mathrm{Sb}$ superlattices,” Phys. Rev. B 57, 6534-6539 (1998).

\title{
2.2 Control of residual background carriers in undoped mid-infrared InAs/GaSb superlattices
}

H. J. Haugan*a, S. Elhamri ${ }^{\text {b }, ~ W . ~ C . ~ M i t c h e l ~}{ }^{\mathrm{a}}$, B. Ullrich ${ }^{\mathrm{c}}$, G. J. Brown ${ }^{\mathrm{a}}$, L. Grazulis ${ }^{\mathrm{a}}$ and S. Houston $^{\mathrm{a}}$

${ }^{\mathrm{a}}$ Air Force Research Laboratory, Wright-Patterson Air Force Base, OH, 45433 USA

${ }^{\mathrm{b}}$ Department of Physics, University of Dayton, OH, 45469 USA

${ }^{\mathrm{c}}$ Department of Physics and Astronomy, Bowling Green State University, Bowling Green, $\mathrm{OH}$ 43403 USA

\begin{abstract}
The performance and operating temperature of infrared (IR) detectors is largely limited by thermal generation and noise processes in the active region of the device. Particularly, excess background charge carriers enhance Auger recombination and dark currents, and depress the detector figures of merit. Therefore, reducing background carriers in the undoped region of pin diodes is an important issue for developing high-operating temperature IR detectors. In this paper, we discuss how, through low-temperature Hall measurements, we optimized several growth-and design parameters to lower residual carrier densities in various mid-IR InAs/GaSb superlattice (SL) designs. Among the growth/processing parameters investigated in the $21 \AA$
\end{abstract}

Distribution Statement A. Approved for public release; distribution unlimited. 
InAs/24 $\AA$ GaSb SLs, neither growth temperature nor in-situ post-growth annealing significantly affected the overall carrier type and density. All of the mid-IR SL samples investigated were residually p-type. The lowest carrier density $\left(1.8 \times 10^{11} \mathrm{~cm}^{-2}\right)$ was achieved in SLs grown at 400 ${ }^{\circ} \mathrm{C}$ and the density was not reduced any further by a post-growth anneal. The growth parameter that most affected the carrier density was interface composition control. With a minor variation in interface shutter sequence, the carrier density dramatically increased from $\sim 2 \times 10^{11}$ to $5 \times 10^{12}$ $\mathrm{cm}^{-2}$, and the corresponding mobility dropped from 6600 to $26 \mathrm{~cm}^{2} / \mathrm{Vs}$, indicating a degradation of interface quality. However, the carrier density was further reduced to $1 \times 10^{11} \mathrm{~cm}^{-2}$ by increasing the GaSb layer width. More importantly, a dramatic improvement on the photoluminescence intensity was achieved with wider GaSb SLs. The disadvantage is that as GaSb layer width increases from 24 to $48 \AA$, the photoluminescence peak position shifts from 4.1 to $3.4 \mu \mathrm{m}$, for a fixed InAs width of $21 \AA$, indicating a photodiode based on these wider designs would fall short of fully covering the 3 to $5 \mu \mathrm{m}$ mid-IR spectral region.

Keywords: Residual carriers, infrared detectors, InAs/GaSb superlattices, molecular beam epitaxy

\section{INTRODUCTION}

Quantum engineered materials and devices that can be exploited for thermoelectrically (TE) cooled mid-infrared (IR) detectors have drawn much attention in recent years due to an increasing demand for low weight and power infrared cameras. ${ }^{1-3}$ Commercially available uncooled IR cameras based on thermal detectors have low detectivities and long integration times, therefore they are not useful in many applications requiring longer range and higher speed detection. ${ }^{3}$ In recent years, several groups have demonstrated pin photodiode arrays using shortperiod InAs/GaSb superlattices (SLs), ${ }^{4,5}$ opening the possibility of their use as TE cooled mid-IR detectors. Despite continuous efforts to improve SL designs and material quality, progress in this detector technology is still slow. To improve detector performance at near room temperature operation, several concepts have been proposed. ${ }^{6,7}$ In the high operating temperature (HOT) detector concept, optimization depends on two key materials parameters: reduced majority carrier concentration and reduced density of Shockley-Read (S-R) centers in the active region. To achieve a wide depletion width $(\sim 5 \mu \mathrm{m})$ with low applied bias, and thus a good absorption quantum efficiency, the majority carrier concentration in the active region of the photodiode should be about $10^{14} \mathrm{~cm}^{-3}$. Unfortunately, for most SL structures fabricated to date, background carrier concentrations of $10^{15}-10^{16} \mathrm{~cm}^{-3}$ are more typical, ${ }^{8}$ and further progress appears slow.

Hall measurements are normally used to characterize the carrier concentration in semiconductor materials. However, since high-quality SLs used in actual devices are typically grown on conducting GaSb substrates, traditional Hall measurements have difficulty separating the conductivity of the SL from that due to the substrate. Many groups have tried to avoid this complication by growing SL layers on semi-insulating GaAs substrates ${ }^{9}$ or by inserting an electrical isolation layer of $\mathrm{AlGaAsSb}^{8}$ to investigate the electrical transport properties. However, these results are often not directly applicable to those of SLs grown on a latticematched GaSb substrate. In our recent report, we demonstrated that low-temperature Hall measurements are effective for characterizing intrinsic carriers in mid-infrared InAs/GaSb SLs grown on GaSb substrates. ${ }^{10}$ At low temperature, the carriers from the substrates freeze out and the characteristics of the carriers in the SLs can be investigated.

Distribution Statement A. Approved for public release; distribution unlimited. 
In this work, we discuss how, through low-temperature Hall measurements, several molecular beam epitaxy (MBE) growth parameters and SL design parameters were tuned to reduce background carrier concentrations in mid-IR InAs/GaSb SLs. In addition, overall interfacial quality was optimized by monitoring changes in the in-plane carrier mobility. Since carrier transport along the in-plane direction is largely affected by intrinsic scattering mechanisms, such as interface roughness scattering (IRS), the in-plane carrier mobility can be used to quantify the SL layer quality and the degree of IRS. We point out that in-plane carrier characteristics measured at low temperatures are not directly related to high-temperature detector performance, where vertical transport is important. However, these mobilities and carrier concentrations can be good indicators of material quality relevant to device performance.

\section{SUPERLATTICE GROWTH}

The InAs/GaSb SL structures were grown by a Varian GEN-II MBE on epi-ready p-type GaSb (100) wafers. A $0.5 \mu \mathrm{m}$-thick GaSb buffer layer was grown at $490{ }^{\circ} \mathrm{C}$ and the substrate temperature was then lowered to the growth temperature $\left(T_{g}\right)$ for the SL layer growth. Since the optical and electrical quality of SLs largely depends on overall material quality, initial growth conditions were tuned to obtain morphologically smooth surfaces. Using a mid-IR SL design of $21 \AA \mathrm{InAs} / 24 \AA \mathrm{GaSb}$, we obtained a low temperature photoresponse onset of $4.13 \mu \mathrm{m}$ (see Figure 4). Most SL samples grown at $390-400{ }^{\circ} \mathrm{C}$ with III/V beam flux ratio of 3 had a featureless surface morphology with an average roughness of $1 \AA$ as shown in a $100 \times 100 \mu \mathrm{m}^{2}$ area scan by atomic force microscopy (Figure 4).

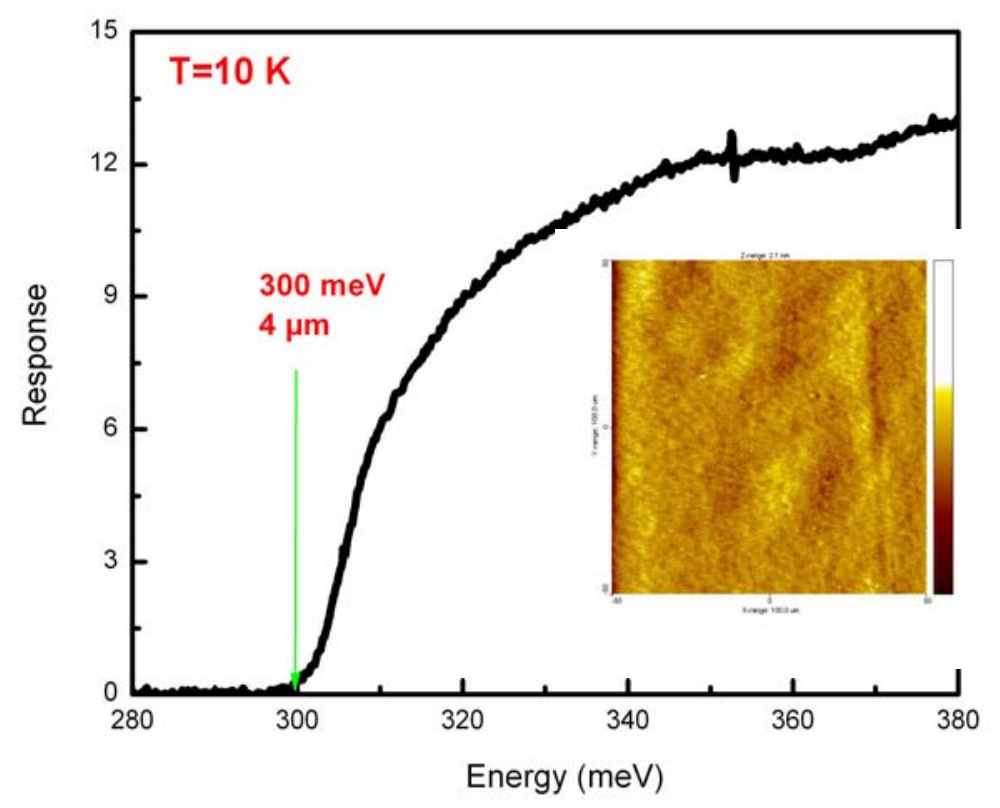

Figure 4. Photoresponse spectrum of the $0.5 \mu$ m-thick $21 \AA$ InAs/24 $\AA$ GaSb superlattices (SLs) taken at $10 \mathrm{~K}$. The insert shows the $100 \mu \mathrm{m} \times 100 \mu \mathrm{m}$ scan by an atomic force microscope of the SL sample.

Most mid-IR SL designs we selected contain 10-20 Å individual layer thicknesses. To grow such thin layers, slow growth rates in general produce much better morphological and optical qualities. ${ }^{11}$ For this study, we selected extremely slow growth rates for the InAs layers and InSb

Distribution Statement A. Approved for public release; distribution unlimited. 
interfaces to control the intended interface accurately. The GaSb layer can be grown slowly as well, however, we chose a slightly faster growth rate for the GaSb layer to have a reasonable growth time for the GaSb buffer layer. With the growth rate of $0.3 \AA / \mathrm{s}(1 \AA / \mathrm{s})$ for the InAs $(\mathrm{GaSb})$ layer, we achieved excellent morphological quality for the majority of the designs to be discussed. Detailed growth conditions can be found elsewhere. ${ }^{11,12}$ Structural parameters of the SLs were determined by high resolution x-ray rocking curves, and the measured SL periods were close to the intended values within the experimental accuracy of $\pm 0.5 \AA$. Measured strains in the SLs ranged from -0.3 to $+0.1 \%$ depending upon the SL designs and interfaces. The strains in the SLs were not intentionally controlled for most of the series, unless otherwise specified in the text.

\section{TRANSPORT MEASUREMENTS}

In order to determine in-plane carrier concentration, mobility and resistivity of each SL sample, temperature-dependent resistivity and single field ( $0.5 \mathrm{~T})$ Hall measurements in a guarded directcurrent system were used. The sample size was roughly $1 \times 1 \mathrm{~cm}^{2}$ with a van der Pauw geometry, and Ohmic contacts were placed on the corners of the samples using indium. Since standard ptype GaSb substrates show significant conduction, the samples were cooled to $10 \mathrm{~K}$ to freeze out the carriers coming from the substrates. To demonstrate this approach, the sheet carrier densities obtained from a SL sample and a bare GaSb substrate are plotted in Figure 5a for comparison. The apparent anomalous increase in the substrate carrier concentration observed at temperatures below $20 \mathrm{~K}$ is an artifact we have observed in all commercial GaSb wafers tested. This artifact is a result of hopping conduction. Hopping conduction occurs when charge carriers tunnel between dopant atoms. This bandless transport results in the vanishing of the Hall voltage for these carriers. The apparent increase in carrier concentration can be shown to be an artifact by a study of two-band conduction. ${ }^{13}$ The actual carriers in the valence band continues to decrease exponentially as the temperature decreases. The sheet resistance of the SL and the GaSb substrate is perhaps more informative. This is shown in Figure 5b, where it can clearly be seen that the substrate resistivity is at least two orders of magnitude higher than that of the SL at temperatures below $20 \mathrm{~K}$. Therefore, we can utilize the $10 \mathrm{~K}$ results to assess the carrier characteristic of the SLs.

Distribution Statement A. Approved for public release; distribution unlimited. 

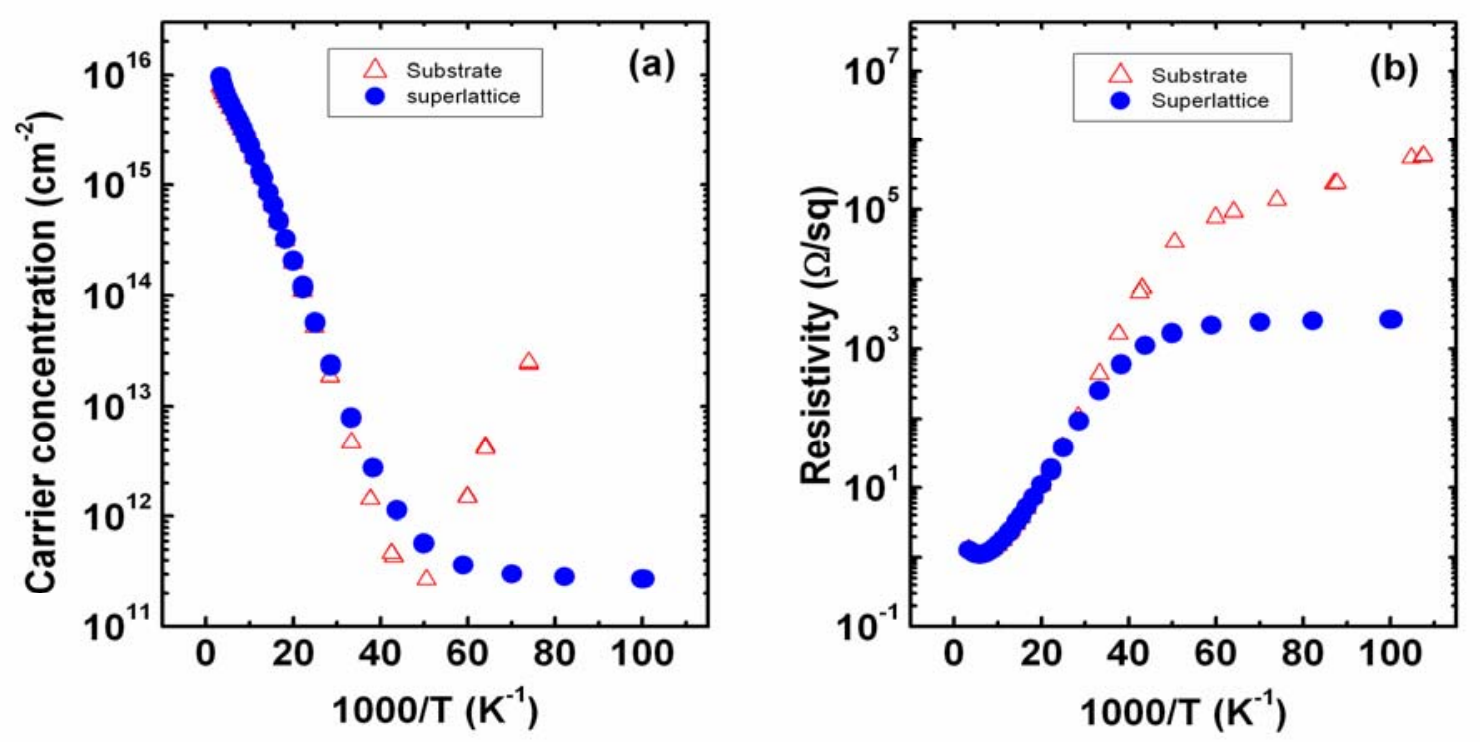

Figure 5. The temperature dependence of (a) the carrier concentrations, (b) resistivity as a function of 1000/T for the $0.5 \mu \mathrm{m}$-thick $21 \AA$ InAs/24 $\AA$ GaSb superlattices (•) on the GaSb substrate and the GaSb substrate $(\Delta)$. Below $20 \mathrm{~K}$, holes in the GaSb substrate show hopping conduction, which is an artifact and not reliable.

\section{OPTIMIZATION OF RESIDUAL CARRIERS}

In order to reduce background carrier concentrations in the SLs, the impact of several growth parameters were investigated. Since growth temperature $\left(\mathrm{T}_{\mathrm{g}}\right)$ controls anion exchange mechanism that affects the residual doping characteristics and the interface roughness, it was the first growth parameter adjusted. Five samples with 110 repeats of $21 \AA$ InAs/ $24 \AA \mathrm{GaSb}$ periods were grown at $370,380,400,410$ and $430{ }^{\circ} \mathrm{C}$. Growth temperatures quoted throughout the paper refer to those measured by a calibrated optical pyrometer referenced at GaSb oxide desorption temperature of $530^{\circ} \mathrm{C}$. Figure 6a plots the $10 \mathrm{~K}$ carrier densities as a function of $\mathrm{T}_{\mathrm{g}}$. The SLs grown at $400{ }^{\circ} \mathrm{C}$ had the lowest density $\left(1.8 \times 10^{11} \mathrm{~cm}^{-2}\right)$. The carrier density slightly increases at $\mathrm{T}_{\mathrm{g}}$ below or above $400{ }^{\circ} \mathrm{C}$, however it remained in the low $10^{11} \mathrm{~cm}^{-2}$ regardless of $\mathrm{T}_{\mathrm{g}}$. It should be noted that the carrier density referred to in the paper is an areal sheet density. As an example, an areal density of $1.8 \times 10^{11} \mathrm{~cm}^{-2}$ for 110 repeated SL periods has an average areal density per period of $1.6 \times 10^{9} \mathrm{~cm}^{-2}$ or, correspondingly, the volume density of the whole SL would be $3.6 \times 10^{15} \mathrm{~cm}^{-3}$. All SL samples in this series were residually $\mathrm{p}$-type, a characteristic which did not change throughout the entire measurement temperature range studied (10-300 K). Although carrier densities show no significant change, hole mobility in the in-plane direction decreased from 8740 to $1400 \mathrm{~cm}^{2} / \mathrm{Vs}$ as $\mathrm{T}_{\mathrm{g}}$ increases from 370 to $430{ }^{\circ} \mathrm{C}$ (see Figure $6 \mathrm{~b}$ ). This decrease in mobility can be explained by an increase in interface roughness. The degree of interface roughness with increasing $\mathrm{T}_{\mathrm{g}}$ was extracted using the Gold model,${ }^{14}$ the details of which can be found in Haugan et. al. ${ }^{10}$ There are few microscopic techniques, such as scanning tunneling microscopy, ${ }^{15}$ that can quantify interfacial quality, however it is almost impossible to use these techniques to optimize daily growth. Therefore, in-plane carrier mobility can be a useful tool to assess interface quality indirectly. To see the effect of $\mathrm{T}_{\mathrm{g}}$ on the morphological qualities, the 3-dimensional atomic force microscope images of the SL sample grown at 370 and

Distribution Statement A. Approved for public release; distribution unlimited. 
$430{ }^{\circ} \mathrm{C}$ were compared. Figure $6 \mathrm{~b}$ insert shows significant differences in surface smoothness; the $\mathrm{SL}$ grown at $430{ }^{\circ} \mathrm{C}$ had a much rougher surface than the one grown at $370{ }^{\circ} \mathrm{C}$.
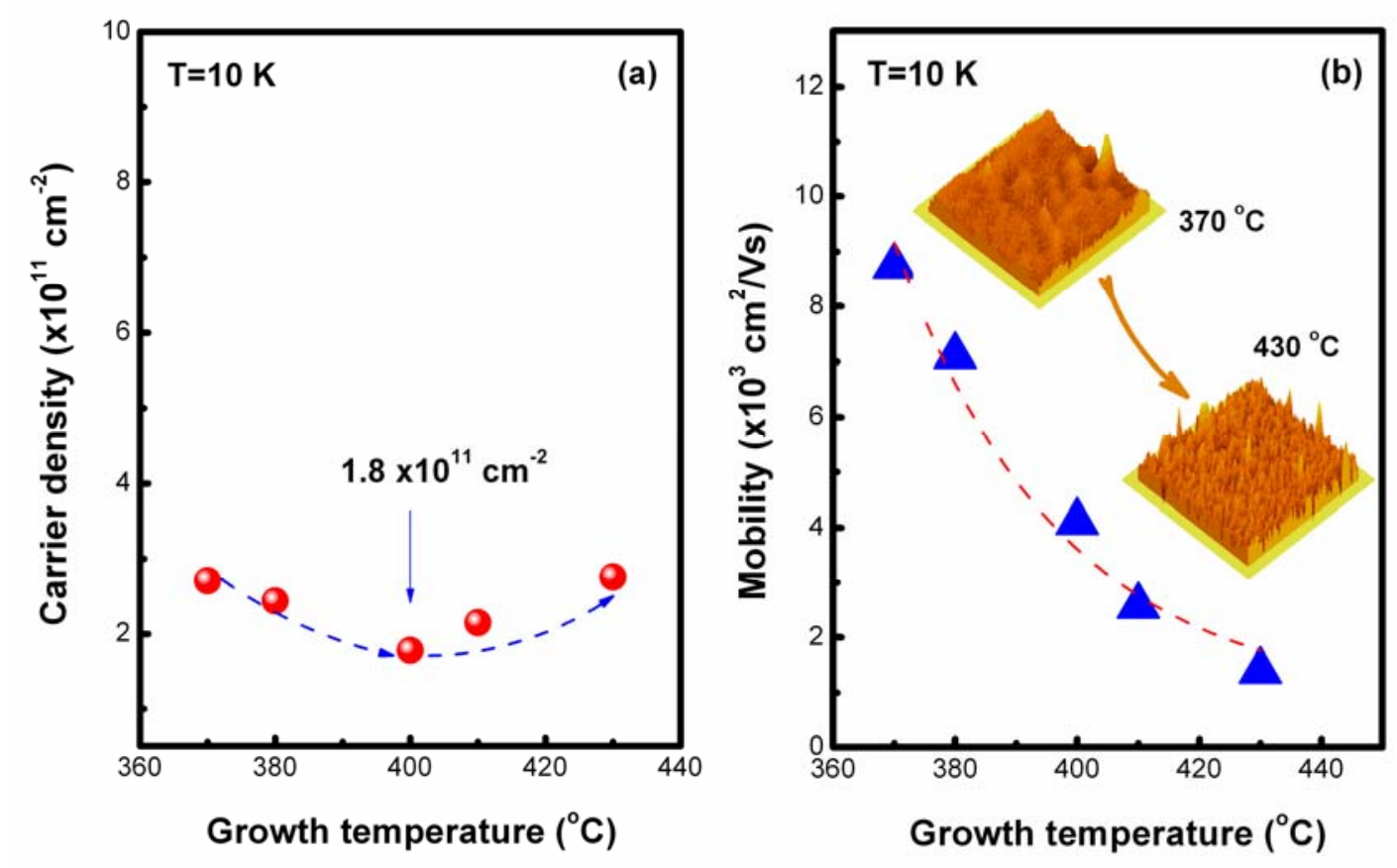

Figure 6. (a) The carrier density $(\bullet)$ and (b) in-plane carrier mobility ( $\Delta)$ comparing SL samples grown at different growth temperatures. The insert shows the 3D AFM images of $20 \times 20 \mu \mathrm{m}$ area scan of the SLs grown at 370 (top) and $430{ }^{\circ} \mathrm{C}$ (bottom) showing significant differences in surface smoothness.

The second parameter investigated was application of post growth annealing to see whether an additional treatment can reduce the residual carrier concentration and/or enhance carrier transport properties. For this study, four SL samples were grown at $400{ }^{\circ} \mathrm{C}$, and then three SLs were annealed at 450,475 and $490{ }^{\circ} \mathrm{C}$ for 15 minutes under an Sb-over pressure immediately after the SL growth. The carrier densities of the annealed samples were then compared to those of the unannealed sample. Figure 7a shows that there is no significant difference between annealed and unannealed samples, all of which showed an average density of $\sim 1.6 \times 10^{11} \mathrm{~cm}^{-2}$ similar to the lowest density obtained from the first series. For the majority carrier mobilities, insitu annealing improves the mobility slightly, especially at higher annealing temperatures (above $450{ }^{\circ} \mathrm{C}$ ), but the factor of 1.5 at most is not considered significant. All annealed SLs in this series remained p-type.

Distribution Statement A. Approved for public release; distribution unlimited. 

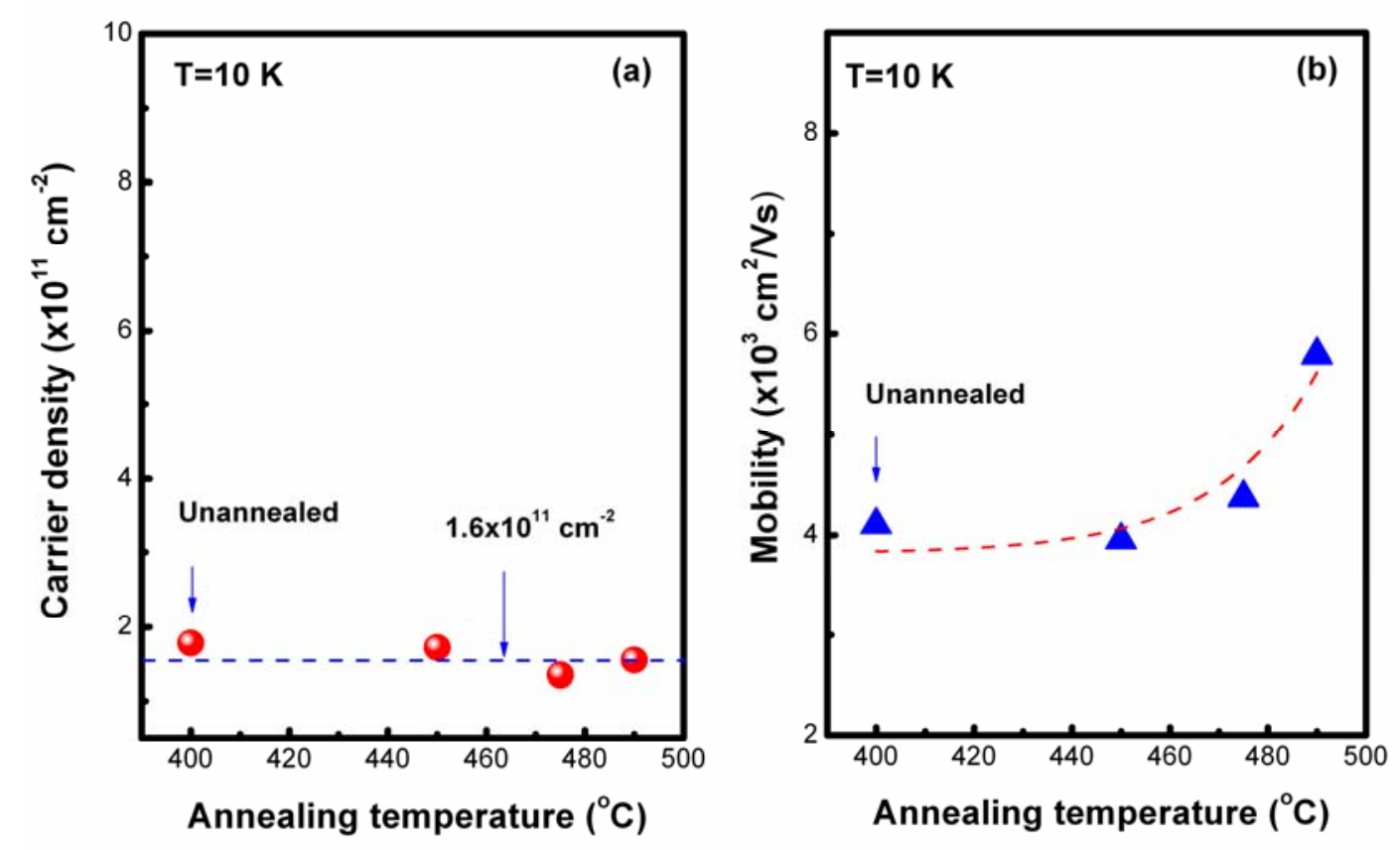

Figure 7. (a) The carrier density (•), (b) in-plane carrier mobility ( $\Delta$ ); comparing SL samples with and without post-annealing. All SL samples had same design of $21 \AA$ InAs/24 Å GaSb superlattices.

The third growth parameter investigated was interface composition control. For this study, four variations of interface shutter sequences were investigated. These were InSb/GaAs, InSb/InSb, $\mathrm{GaAs} / \mathrm{GaAs}$ and $\mathrm{GaAs} / \mathrm{InSb}$. The order of interface formation is written from left to right. For example, to form the InSb/GaAs interface sequence immediately after GaSb layer growth, the $\mathrm{Ga}$ shutter was closed while Sb-flux continued for 1 second; $0.3 \AA$ of indium (1 s) was then deposited without Sb-flux, and InAs layer growth was then continued. After InAs layer growth, the Indium shutter was closed while As-flux continued for $1 \mathrm{~s}$, after which $0.3 \AA$ of gallium $(0.5$ s) was deposited without As-flux and GaSb layer growth was continued. Without intentional interface control (noted as 0/0), InSb-like interfacial bonds naturally form for InAs-on-GaSb, while GaAs-like bonds are likely for GaSb-on-InAs, resulting in $-0.13 \%$ strain. The amounts of interface mentioned here are estimated values from shutter sequence time and, more practically, will be mixed quaternary alloys of $\mathrm{In}_{\mathrm{x}} \mathrm{Ga}_{1-\mathrm{x}} \mathrm{Sb}_{\mathrm{y}} \mathrm{As}_{1-\mathrm{y}}$. All SLs in this series were grown at $400{ }^{\circ} \mathrm{C}$. The measured structural and electrical parameters are summarized in Table 1. The table shows that the measured periods of all five SLs were around $45 \AA$ as intended. However, the strain and the in-plane mobility were very sensitive to the minor variation of interface shutter sequences. Since an InSb-like (GaAs-like) interface would relieve tension (compression), the combination of both types of interfaces can be used profitably to balance strain in the SLs. For our series, by inserting two InSb (GaAs)-like interfaces, the $-0.13 \%$ of strain was able to be adjusted to +0.10 $\%(-0.3 \%)$. The strain values with different combination of interface types can be found in Table 3. For the interface abruptness, among the four interface types described in Table 3, the $\mathrm{InSb} / \mathrm{GaAs}$ is the most desirable and the $\mathrm{GaAs} / \mathrm{InSb}$ the least desirable interface sequence, because it is the former that promotes natural interfacial bonds on both sides. This is demonstrated from the result shown in Table 3, where we observe a drastic reduction in the inplane mobility of the $\mathrm{GaAs} / \mathrm{InSb}$ interface sequence $\left(26 \mathrm{~cm}^{2} / \mathrm{Vs}\right)$, whereas the others show a

Distribution Statement A. Approved for public release; distribution unlimited. 
moderate improvement in comparison to the uncontrolled sequence $0 / 0\left(4100 \mathrm{~cm}^{2} / \mathrm{Vs}\right)$. With the $\mathrm{GaAs} / \mathrm{InSb}$ interface sequence, the background carrier density increased from the low $10^{11} \mathrm{~cm}^{-2}$ to $5 \times 10^{12} \mathrm{~cm}^{-2}$, demonstrating the impact of interface shutter sequence on the quality of interfaces, and thus carrier concentration and mobility. Figure 8 summarizes the effect of different interface shutter sequences on the properties of carriers. All SLs with controlled/uncontrolled interfaces were p-type.

Table 3. Summary of the results obtained from multi-carrier analysis of the data from 21 $\AA($ InAs+IF)/24 $\AA($ GaSb+IF) superlattices. The $0.3 \AA$ of InSb-like or/and GaAs-like interfaces (IF) were inserted between the layers and their values were estimated from shutter time. The $0 / 0$ represents uncontrolled IF. The Rs, ns and $\mu$ represent the $10-K$ resistivity, hole density and in-plane hole mobility.

\begin{tabular}{|c|c|c|c|c|c|}
\hline Interface type & Period $\AA$ & Strain $\%$ & $\mathbf{R}_{\mathbf{s}} \mathbf{\Omega} / \mathbf{s q}$ & $\mathbf{n}_{\mathbf{s}} \mathbf{x} \mathbf{1 0}^{\mathbf{1 1}} \mathbf{c m}^{-\mathbf{2}}$ & $\boldsymbol{\mu} \mathbf{~ c m}^{\mathbf{2}} / \mathbf{V} \mathbf{~}$ \\
\hline $0 / 0$ & 44.8 & -0.13 & 8563 & 1.8 & 4104 \\
\hline $\mathrm{InSb} / \mathrm{GaAs}$ & 45.4 & -0.08 & 3409 & 2.8 & 6579 \\
\hline $\mathrm{InSb} / \mathrm{InSb}$ & 45.5 & +0.10 & 7020 & 2.0 & 4343 \\
\hline $\mathrm{GaAs} / \mathrm{GaAs}$ & 45.2 & -0.30 & 7675 & 1.5 & 5548 \\
\hline $\mathrm{GaAs} / \mathrm{InSb}$ & 45.1 & -0.15 & 45690 & 52.2 & 26 \\
\hline
\end{tabular}

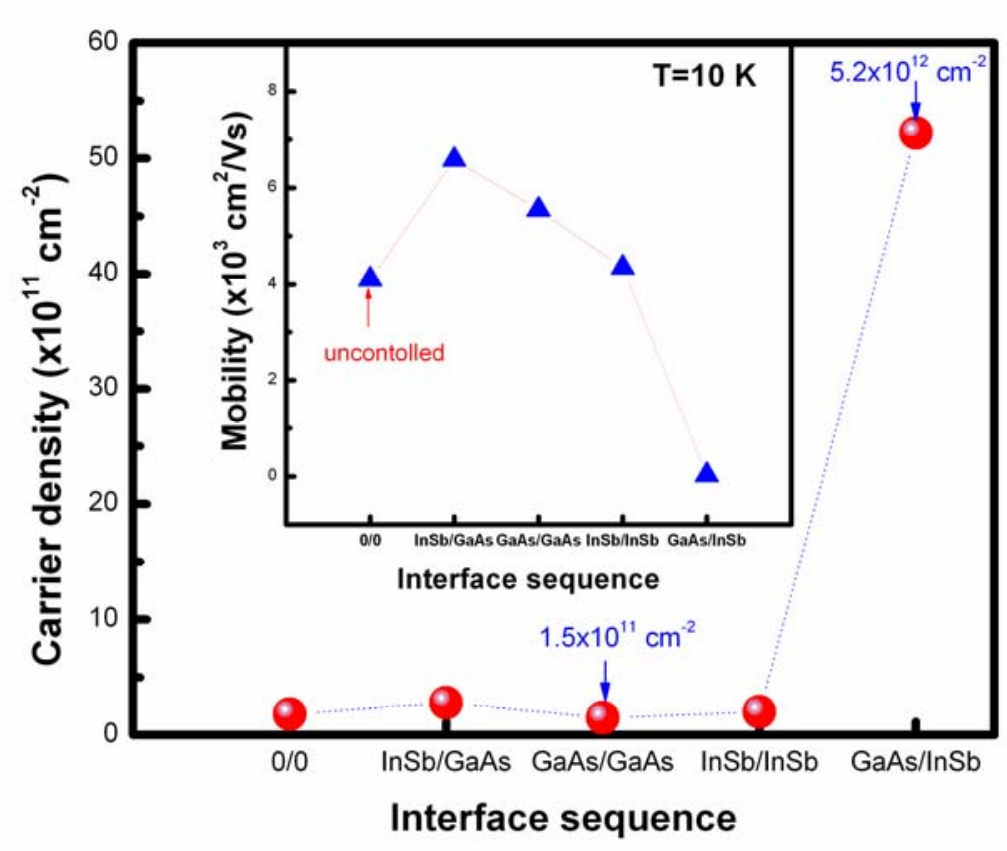

Figure 8. The carrier density $(\bullet)$ and in-plane mobility $(\Delta)$ of the $21 \AA(\operatorname{InAs}+\mathrm{IF}) / 24 \AA$ $(\mathrm{GaSb}+\mathrm{IF})$ superlattices with four variations of interface shutter sequences; InSb/GaAs, InSb/InSb, GaAs/GaAs, and GaAs/InSb comparing with those of uncontrolled sequence $\mathbf{0} / \mathbf{0}$.

Distribution Statement A. Approved for public release; distribution unlimited. 
Finally, in order to improve material quality for better electrical/optical response, we adapted a different buffer growth procedure reported in the literature, ${ }^{16}$ in which the last part of the buffer layer was grown at gradually decreasing temperature $\left(490\right.$ to $\left.400{ }^{\circ} \mathrm{C}\right)$. The Hall mobility (8285 $\mathrm{cm}^{2} / \mathrm{Vs}$ ) and photoluminescence (PL) intensity of $21 \AA$ InAs/24 $\AA$ GaSb SLs grown on the new buffer were twice as large as for the SL grown on a GaSb buffer that was entirely grown at 490 ${ }^{\circ} \mathrm{C}\left(4104 \mathrm{~cm}^{2} / \mathrm{Vs}\right)$, while carrier concentration stayed the same $\left(1.7 \times 10^{11} \mathrm{~cm}^{-2}\right)$. Figure 9 shows significant improvements on the in-plane mobility and PL intensity simply by changing buffer growth procedure.
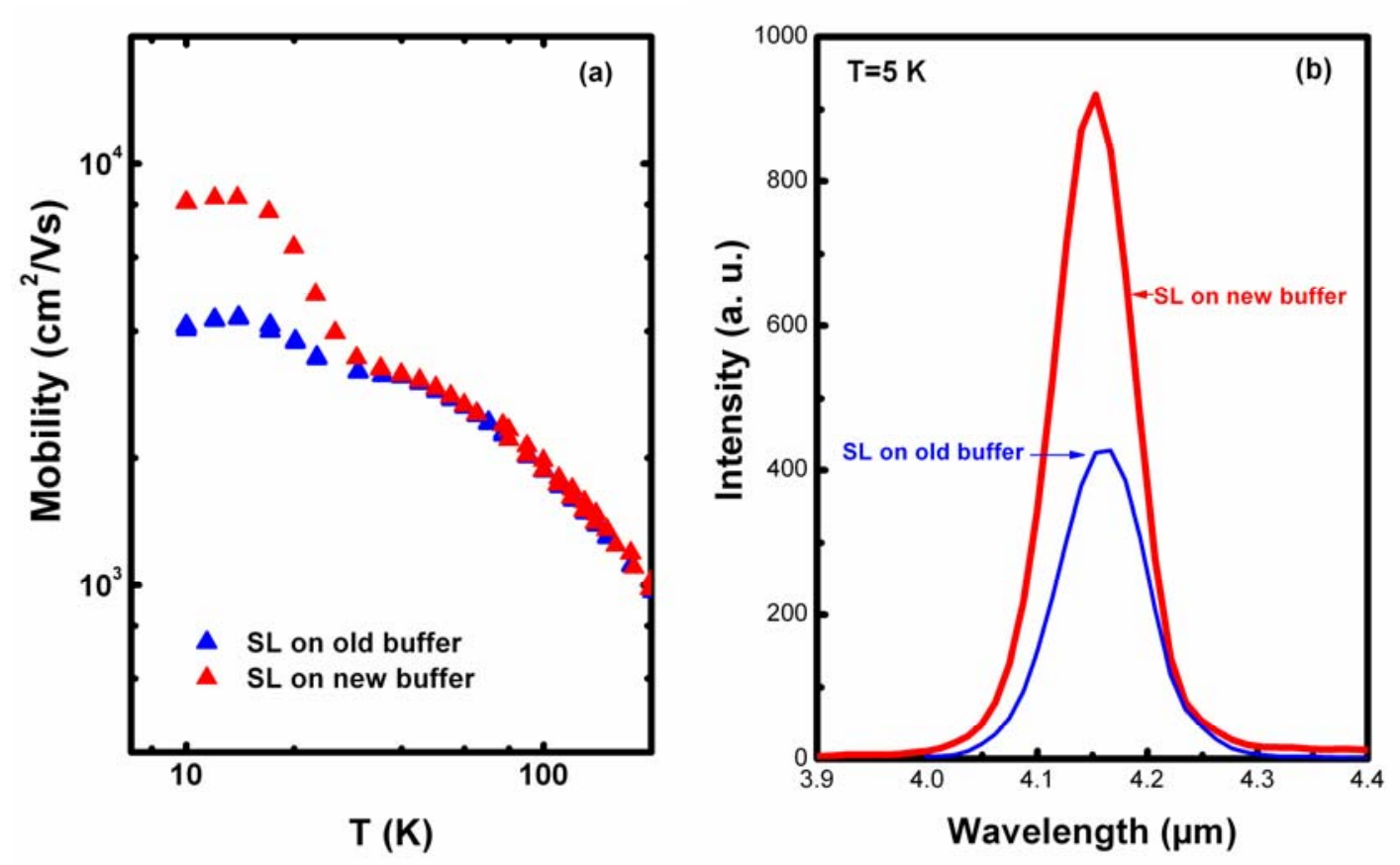

Figure 9. (a) The Hall mobility and (b) PL intensity of $21 \AA$ InAs/24 $\AA$ GaSb superlattices (SLs) grown on old (blue) and new buffer (red). The Hall mobility and PL intensity of the SLs grown on the new buffer that was grown at gradually decreasing temperatures of 490$400{ }^{\circ} \mathrm{C}$ were twice as large as for the SL grown on the old GaSb buffer that was entirely grown at $490{ }^{\circ} \mathrm{C}$.

Since we were able to improve Hall mobility and PL intensity using new buffer growth procedure mentioned the above, the last series of SL samples was grown with the GaSb layer widths of 24, 36, $48 \AA$ at the fixed InAs layer width of $21 \AA$, in order to study the effect of barrier width on the residual carriers and the PL emission. Since the PL intensity is sensitive to the presence of any nonradiative processes, strong PL can relate to high material quality. In addition, the excitonic-PL linewidth provides information on the spatial distribution of the roughness at the hetero-interfaces. ${ }^{17}$ A single narrow peak can relate to high interfacial quality. Smooth interfaces also improve the overall in-plane carrier transport. In order to perform PL measurements at cryogenic temperatures, the SL samples were mounted in a helium cooled optical cryostat. For the excitation, the continuous wavelength second harmonic emission at 532 $\mathrm{nm}$ of a Neodymium Vanadate laser was employed. The spectral emission intensity was measured with a nitrogen cooled InSb detector attached to a Bomem Fourier-transform infrared spectrometer. Table 4 summarizes the Hall and PL data, and Figure 10 displays the $5 \mathrm{~K}$ PL

Distribution Statement A. Approved for public release; distribution unlimited. 
spectra of $21 \AA$ InAs/ X $\AA$ GaSb SLs, where X=24, 36 and $48 \AA$. All SLs in the last series were grown at $400{ }^{\circ} \mathrm{C}$.

Table 4. Hall and photoluminescence (PL) data for the InAs/GaSb superlattices. The InAs width was fixed at $21 \AA$. All SLs in the series were p-type. Three parameters $R, n$, and $\mu$ represent the resistivity, hole concentration, and mobility respectively.

\begin{tabular}{|c|c|c|c|c|c|c|}
\hline \multirow[b]{2}{*}{ GaSb $(\AA ̊)$} & \multicolumn{3}{|c|}{10 K Hall } & \multicolumn{3}{|c|}{$5 \mathrm{~K} \mathrm{PL}$} \\
\hline & $\begin{array}{c}\mathbf{R} \\
(\Omega / \mathbf{s q})\end{array}$ & $\begin{array}{c}\mathbf{N} \\
\left(\mathrm{x10}^{11} \mathrm{~cm}^{-2}\right)\end{array}$ & $\begin{array}{c}\mu \\
\left(\mathrm{cm}^{2} / \mathrm{Vs}\right)\end{array}$ & $\begin{array}{l}\text { Peak } \\
(\mu \mathrm{m})\end{array}$ & $\begin{array}{l}\text { Int. } \\
\text { (a. u.) }\end{array}$ & $\begin{array}{c}\text { FWHM } \\
\text { (meV) }\end{array}$ \\
\hline 24 & 4320 & 1.74 & 8285 & 4.1 & 910 & 5.3 \\
\hline 36 & 10928 & 0.99 & 5717 & 3.6 & 3342 & 4.9 \\
\hline 48 & 14963 & 1.17 & 3517 & 3.4 & 3612 & 4.2 \\
\hline
\end{tabular}

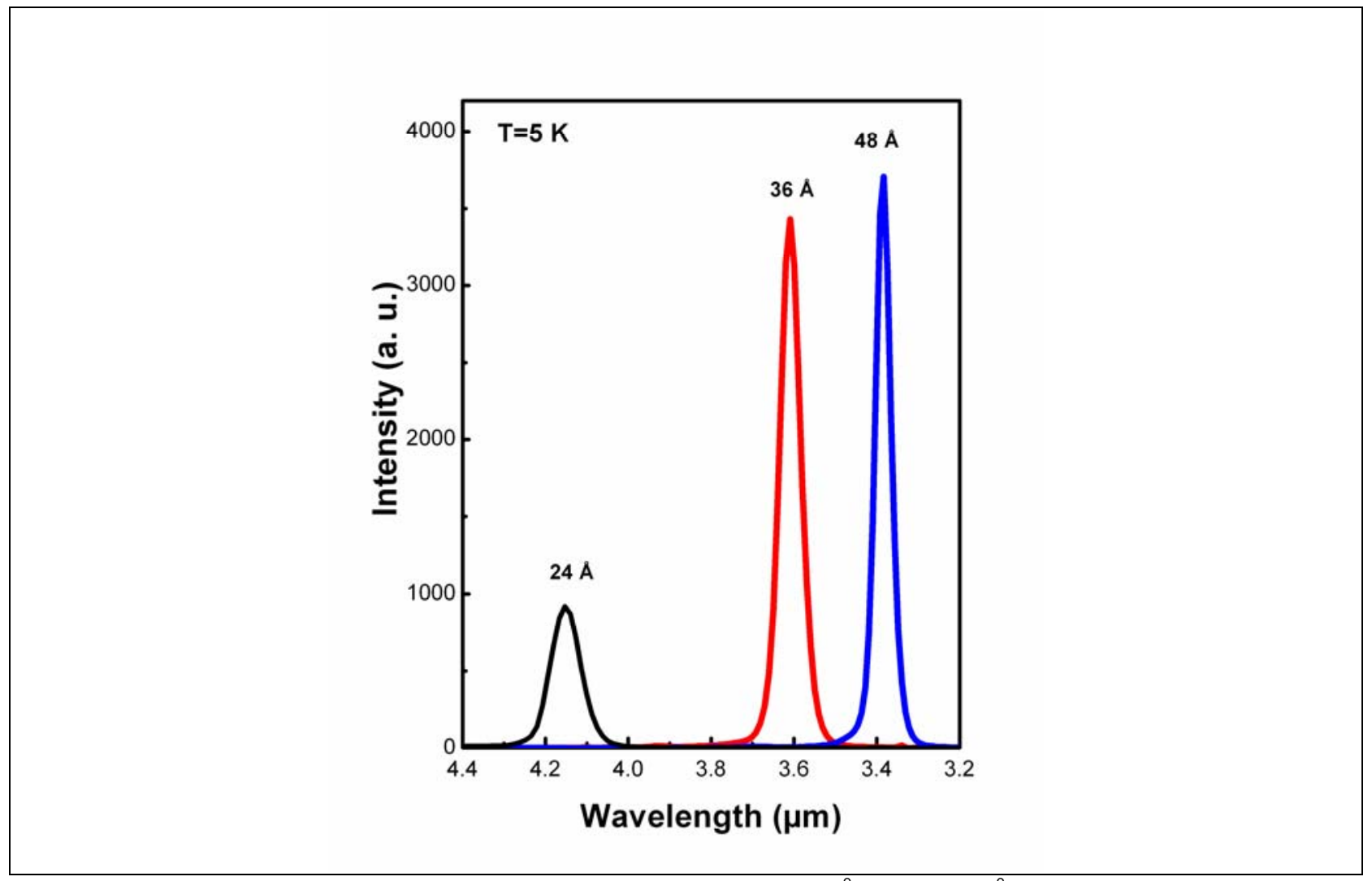

Figure 10. The $5 \mathrm{~K}$ photoluminescence spectra of $21 \AA \AA \operatorname{InAs} / \mathrm{X} \AA \mathrm{GaSb}$ superlattices, where $X=24,36$ and $48 \AA$ A.

As the GaSb layer width doubles from 24 to $48 \AA$ : (1) the PL peak position decreased from 4.1 to $3.4 \mu \mathrm{m}$, to the edge of the mid-IR atmospheric window; (2) the majority carriers were holes, and their density and mobility decreased, resulting in an almost four-fold increase in the sheet resistivity. The lower carrier densities were obtained from the SLs with the wider GaSb widths; (3) the PL intensity increased and the full width at half maximum (FWHM) of the PL peak decreased to almost $4 \mathrm{meV}$. The FWHM below $5 \mathrm{meV}$ correlates with excellent optical response and high interfacial quality.

Distribution Statement A. Approved for public release; distribution unlimited. 


\section{CONCLUSIONS}

Several molecular beam epitaxy growth parameters were optimized to obtain the lowest residual carriers in mid-infrared (IR) $21 \AA$ InAs/ $24 \AA$ GaSb SLs designed for a $4 \mu \mathrm{m}$ cutoff wavelength. Among the parameters studied, neither growth temperature nor in-situ post-annealing affected the intrinsic carrier type and concentration significantly. Over the $370-430{ }^{\circ} \mathrm{C}$ growth temperature range studied, the lowest carrier density of $1.8 \times 10^{11} \mathrm{~cm}^{-2}$ was obtained from the SL grown at $400{ }^{\circ} \mathrm{C}$ and overall densities stay in the low $10^{11} \mathrm{~cm}^{-2}$ regardless of growth temperature. All SLs grown at $400{ }^{\circ} \mathrm{C}$ had low carrier densities with or without annealing, while in-plane carrier mobility improved slightly with annealing at higher annealing temperatures of 450-490 ${ }^{\circ} \mathrm{C}$. The growth parameter that affected the carrier density the most was interface-control. With a minor variation in interface shutter sequence, the carrier density increased from $\sim 2 \times 10^{11}$ to $5 \times 10^{12} \mathrm{~cm}^{-2}$, and the corresponding mobility dropped from 6600 to $26 \mathrm{~cm}^{2} / \mathrm{Vs}$; indicating dramatic degradation of interface quality. All of the mid-infrared superlattices investigated in the studies were p-type. Lowering carrier densities to $1 \times 10^{11} \mathrm{~cm}^{-2}$ was achieved by increasing the GaSb layer width. By doubling the GaSb layer width from 24 to $48 \AA$, the photoluminescence peak wavelength shifted from 4.1 to $3.4 \mu \mathrm{m}$. More importantly, a dramatic improvement on PL intensity and the full with at half maximum was achieved.

\section{REFERENCES}

1. A. Rogalski, Infrared Phys. Technol. 50, 240 (2007).

2. L. Becker, Proc. SPIE. 6127, 61270S (2006).

3. T. Ashley, N. T. Gordon, Proc. SPIE. 5359, 89 (2004).

4. M. Razeghi, Y. Wei, A. Hood, D. Hoffman, B. M. Nguyen, P. Y. Delaunay, E. Michel, R. McClintock, Proc. SPIE. 6206, 62060N (2006).

5. M. Walther, J. Schmitz, R. Rehm, S. Kopta, F. Fuchs, J. Fleiner, W. Cabanski, J. Ziegler, J. Cryst. Growth 278, 156 (2005); M. Walther, G. Weimann, Phys. Stat. Sol. (a) 203, 3545 (2006).

6. Piotrowski, W. Galus, and M. Grudzień, Infrared Phys. 31, 1 (1991)

7. M. Kinch, Proc. SPIE. 4454, 168 (2001); M. Kinch, Proc. SPIE. 4288, 254 (2001).

8. L. Bürkle, F. Fuchs, J. Schmitz, W. Pletschen, Appl. Phys. Lett. 77, 1659 (2000).

9. C. A. Hoffman, J. R. Meyer, E. R. Youngdale, F. J. Bartoli, and R. H. Miles, Appl. Phys. Lett. 63, 2210 (1993).

10. H. J. Haugan, S. Elhamri, F. Szmulowicz, B. Ullrich, G. J. Brown, W. C. Mitchel, Appl. Phys. Lett. 92, 071102 (2008).

11. H. J. Haugan, K. Mahalingam, G. J. Brown, W. C. Mitchel, B. Ullrich, L. Grazulis, S. Elhamri, J. C. Wickett, D. W. Stokes, J. Appl. Phys. 100, 123110 (2006).

12. H. J. Haugan, L. Grazulis, G. J. Brown, K. Mahalingam, D. H. Tomich, J. Cryst. Growth 261, 471 (2004).

13. W. C. Mitchel, A. O. Evwaraye, S. R. Smith, and M. D. Roth, J. Electron. Mater. 26, 113 (1997).

14. A. Gold, Phys. Rev. B 35, 723 (1987); A. Gold, J. Appl. Phys. 103, 143718 (2008).

15. B. Z. Nosho, W. Barvosa-Carter, M. J. Yang, B. R. Bennett, L. J. Whitman, Surf. Science 465, 361 (2000).

Distribution Statement A. Approved for public release; distribution unlimited. 
16. J. P. Prineas, Mikhail Maiorov, C. Cao, J. T. Olesberg, M. E.Flatté, M. Reddy, C. Coretsopoulos, and M. Itzler, Proc. SPIE. 6119, 611904 (2006).

17. A. P. Ongstad, G. C. Dente, M. L. Tilton, D. Gianardi, and G. Turner, J. Appl. Phys. 87, $7896(2000)$.

\title{
2.3 Type-II Superlattice Materials Research at the Air Force Research Laboratory
}

G. J. Brown ${ }^{1}$, S. Elhamri ${ }^{2}$, H. E. Smith ${ }^{1,3}$, K. Mahalingam ${ }^{1,4}$, H. J. Haugan ${ }^{1,4}$, S. Pacley ${ }^{1}$, B. Ullrich $^{4}$, and F. Szmulowicz ${ }^{1,3}$

${ }^{1}$ Air Force Research Laboratory, Wright-Patterson AFB, OH, USA 45433-7707;

${ }^{2}$ University of Dayton, Dayton, OH, USA 45469

${ }^{3}$ University of Dayton Research Institute, Dayton, OH, USA 45469-0178

${ }^{4}$ Universal Technology Corporation, Dayton, OH, USA 45432-2600

${ }^{5}$ Bowling Green State University, Bowling Green, $\mathrm{OH}$

\begin{abstract}
Type-II superlattice (SL) materials research in the Materials \& Manufacturing Directorate of the Air Force Research Laboratory began in 1988. This materials system holds great promise as the III-V equivalent to HgCdTe alloys for infrared detection. Great progress has been made on the epitaxial growth of InAs $/ \mathrm{Ga}_{1-\mathrm{x}} \mathrm{In}_{\mathrm{x}} \mathrm{Sb}$ superlattices in the past twenty years by a number of research groups. However, not all of the materials issues have been solved. To continue to resolve these limiting materials issues, basic superlattice materials, without photodiode fabrication, are used to characterize the impact of growth processes and SL design on the structural, electrical and optical properties. An integrated approach of theoretical modeling, inhouse molecular beam epitaxy, and a host of materials measurement techniques is employed to study the optimization of the superlattices for infrared detection. In the past few years the majority of the samples grown in-house have been designed for the middle wavelength infrared (MWIR) band. However, there are challenges in applying MWIR SL growth optimization to longer wavelength SLs. Recent progress on understanding the complex interplay between InAs/GaSb superlattice composition and fundamental electrical and optical properties will be covered.
\end{abstract}

Keywords: InAs/GaSb, superlattices, infrared detectors

\section{INTRODUCTION}

Smith and Mailhiot reported in $1987^{1}$ a theoretical comparison of the absorption coefficient of an InAs/InGaSb superlattice to that of a $\mathrm{HgCdTe}$ alloy with a $10 \mu \mathrm{m}$ cut-off wavelength. This theoretical prediction of comparable absorption coefficients renewed interest in the InAs/GaSb superlattices first proposed by L. Esaki et al in 1977 for infrared detection. ${ }^{2}$ By 1988 the Materials and Manufacturing Directorate of the Air Force Research Laboratory, began research on this new material system, with the purchase of a Varian Gen II molecular beam epitaxy (MBE) system. Much of the early research emphasis was in two areas: the epitaxial conditions for depositing high crystalline quality superlattices, and theoretical modeling of superlattice designs for absorption in long wavelength and very long wavelength infrared (VLWIR) bands. ${ }^{3,4}$ Since that time tremendous progress has been made by multiple groups on the development for type-II superlattices for infrared detection in wavelength bands from mid-infrared wavelengths (MWIR) to VLWIR. ${ }^{5-12}$ Because of the wide range of wavelengths that the InAs/GaInSb

Distribution Statement A. Approved for public release; distribution unlimited. 
superlattices can be designed to cover, their large absorption coefficient, and the capability for use in photodiodes, they represent a III-V equivalent of the II-VI HgCdTe alloy system.

Two areas where the type-II superlattices are of particular interest are large format MWIR (3-5 $\mu \mathrm{m})$ and VLWIR $(>15 \mu \mathrm{m})$ sensing. In both cases, the possibility of increasing the detector operating temperature above the current state-of-the art in each of these bands is the driving force. For the MWIR band, a binary/binary superlattice of InAs and GaSb layers is used. For the VLWIR band, a binary/ternary system, as proposed by Smith and Mailhiot, is preferred to improve infrared absorption and reduce Auger recombination. ${ }^{13}$ In recent years we have emphasized superlattice designs for the MWIR. ${ }^{14-18}$ Now we are exploring the bridge between MWIR optimization and VLWIR optimization by simply increasing the InAs layer width, while maintaining a constant GaSb width. As several groups have shown, increasing the InAs thickness from $16 \AA$ to $70 \AA$ can rapidly decrease the superlattice energy band gap, or equivalently extend the cut-off wavelength (see Figure 11). ${ }^{19-21}$ However, as the InAs width is increased more is being changed in the superlattice properties than just the band gap energy. Some of the changes that occur will be explored in the following sections.

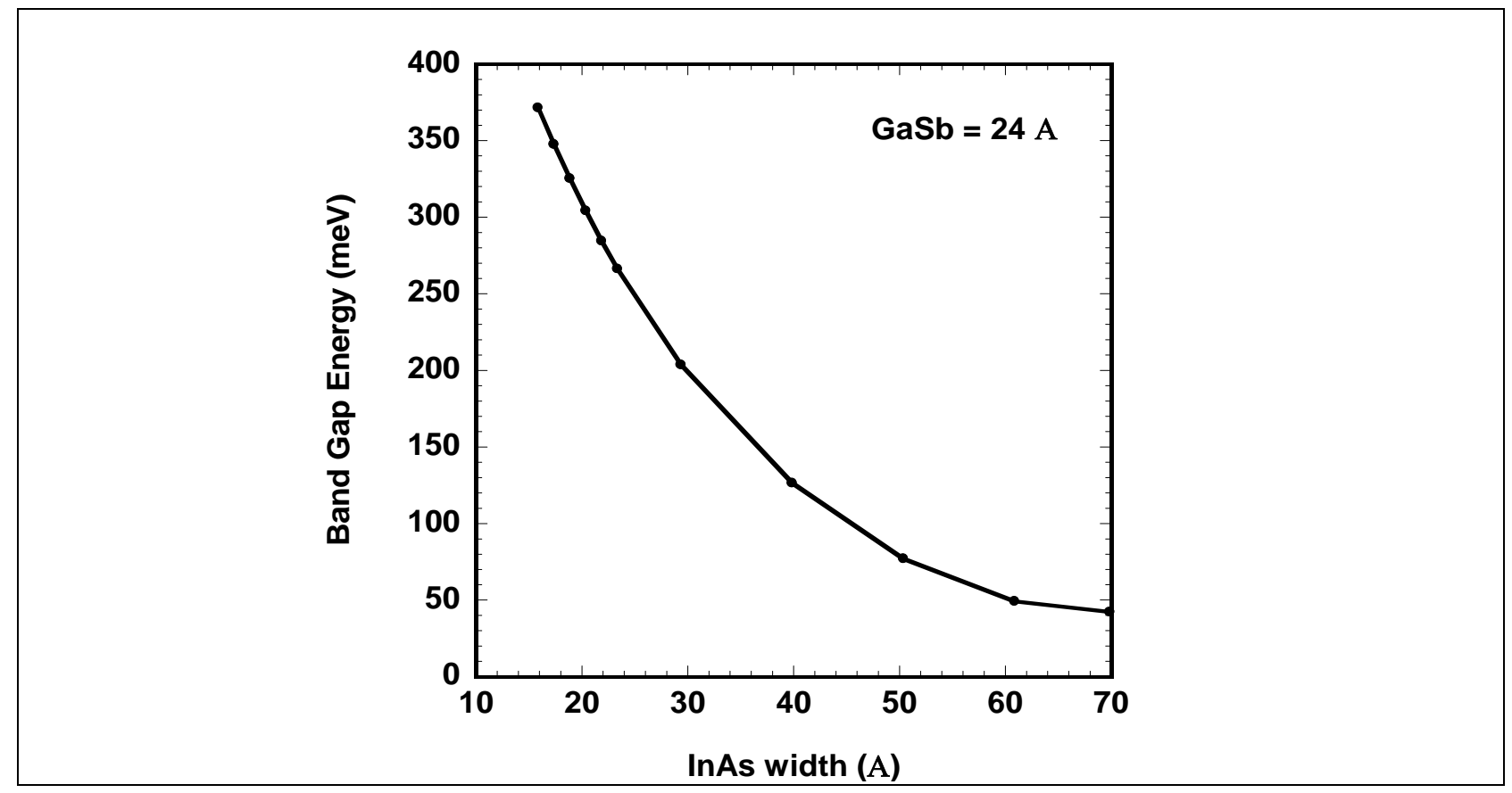

Figure 11. Calculated energy InAs/GaSb Superlattice Band Gap as a Function of InAs Layer Width for a Fixed GaSb Width of $24 \AA$

\section{RESEARCH CAPABILITIES}

The InAs/GaSb SL structures were grown by molecular beam epitaxy, using a Varian GEN-II MBE system, on epi-ready n-type GaSb:Te (100) wafers. A $0.3 \mu \mathrm{m}$-thick GaSb buffer layer was grown at $490{ }^{\circ} \mathrm{C}$ and the substrate temperature was then lowered to about $400{ }^{\circ} \mathrm{C}$ for the superlattice growth. There were two series of samples grown at different times, where the GaSb layer width was kept fixed, while the InAs layer width was increased to shift the cutoff wavelength from the MWIR to the VLWIR range. In the first series, the GaSb width was $26 \AA$

Distribution Statement A. Approved for public release; distribution unlimited. 
while the InAs width was progressively increased: $21,30,40$ and $55 \AA$. In the second series, the GaSb width was $21.5 \AA$ and the InAs widths were: $27.5,34.5,39.5$ and $48.5 \AA$. The total thickness of the superlattice was kept at $0.5 \mu \mathrm{m}$ for each of the SL designs. Since the optical and electrical quality of SLs largely depends on overall material quality, the initial growth conditions were adjusted to obtain smooth surfaces exhibiting step flow growth. The sample surfaces were characterized by Atomic Force Microscopy and were found to have a featureless surface morphology with an average roughness of $1.5 \AA$ over a $100 \times 100 \mu \mathrm{m}^{2}$ scan area.

In the Materials and Manufacturing Directorate we have an extensive array of materials characterization techniques that were developed over the years to study impurities and defects in a variety of bulk and heterostructure semiconductor materials. In order to improve the properties of materials one must be able to measure the properties, whether crystal structure, chemical composition, electrical or optical, and identify/eliminate what is limiting those properties. To this end we can employ fifteen different measurement techniques, each providing a different insight into the nature of the material under study. For crystal structure insight there is high resolution x-ray diffraction, atomic force microscopy and high resolution cross-sectional transmission electron microscopy. For surface chemistry there is auger electron spectroscopy, $\mathrm{x}$ ray photoelectron spectroscopy, and secondary ion mass spectroscopy (SIMS). Several of these techniques can also be used to identify the chemical composition of the materials, such as High Resolution X-ray Diffraction (HRXRD), High Resolution Transmission Electron Microscopy (HRTEM) and SIMS depth profiling. For electrical properties, variable temperature Hall Effect measurements are used, as well as variable field Hall Effect and Shubnikov de Haas measurements. Defect levels can be identified via various capacitance techniques such as Deep Level Transient Spectroscopy, Thermal Admittance Spectroscopy and Optical Admittance Spectroscopy. For optical properties, the key techniques are photoluminescence (PL), photoconductivity (PC) and infrared absorption. Some examples of the insight gained from these techniques when applied to superlattices, with special emphasis on wide InAs layers, will be presented.

\section{RESULTS AND DISCUSSION}

To make a direct comparison between MWIR optimization and VLWIR optimization a couple of the higher photoresponse SL designs were selected as the MWIR starting point. In one series the GaSb width was kept constant at $26 \AA$ while the InAs width was increased from $21 \AA$ to $55 \AA$. In another series the GaSb width was kept constant at $21.5 \AA$ while the InAs width was increased from $27.5 \AA$ to $48.5 \AA$ in smaller increments. In the first series, there was no measurable photoresponse signal for InAs widths greater than $40 \AA$, so the second series was only extended to $48.5 \AA$. For the first series, both photoluminescence and photoconductivity spectra were collected to check the actual shift in band gap energy. The spectra were collected on two different FTIR spectrometers: a Digilab FTS 6000 FTIR was used for the PC spectra, from $10 \mathrm{~K}$ to $100 \mathrm{~K}$, and a Bomem DA3 FTIR was used for the PL measurements at $5 \mathrm{~K}$. In order to collect PL emission beyond $5 \mu \mathrm{m}$ a modulation technique was employed to eliminate the background room temperature blackbody emission of the spectrometer. ${ }^{22}$ The normalized PL and PC spectra are compared in Figure 12. For the $21 \AA \AA$ InAs and the $30 \AA \AA$ InAs samples there is good agreement between the PL peak position and full-width at half-maximum with the PC band edge and width. Typically the PL band gap related transition falls right along the PC band edge transition. This position is above the band gap energy determined by the onset of the

Distribution Statement A. Approved for public release; distribution unlimited. 
photoresponse spectrum. There is a discrepancy between the PL peak position and the photoresponse band edge for the $40 \AA \AA$ wide InAs SL sample. The band gap energy determined from the photoresponse spectrum is closer to the theoretically calculated band gap (with a GaSb width of $24 \AA$ ), $148 \mathrm{meV}$ compared to $126.6 \mathrm{meV}$, than the PL peak position at $172 \mathrm{meV}$. Perhaps this discrepancy highlights why employing multiple techniques can provide a cross check on measurement results.

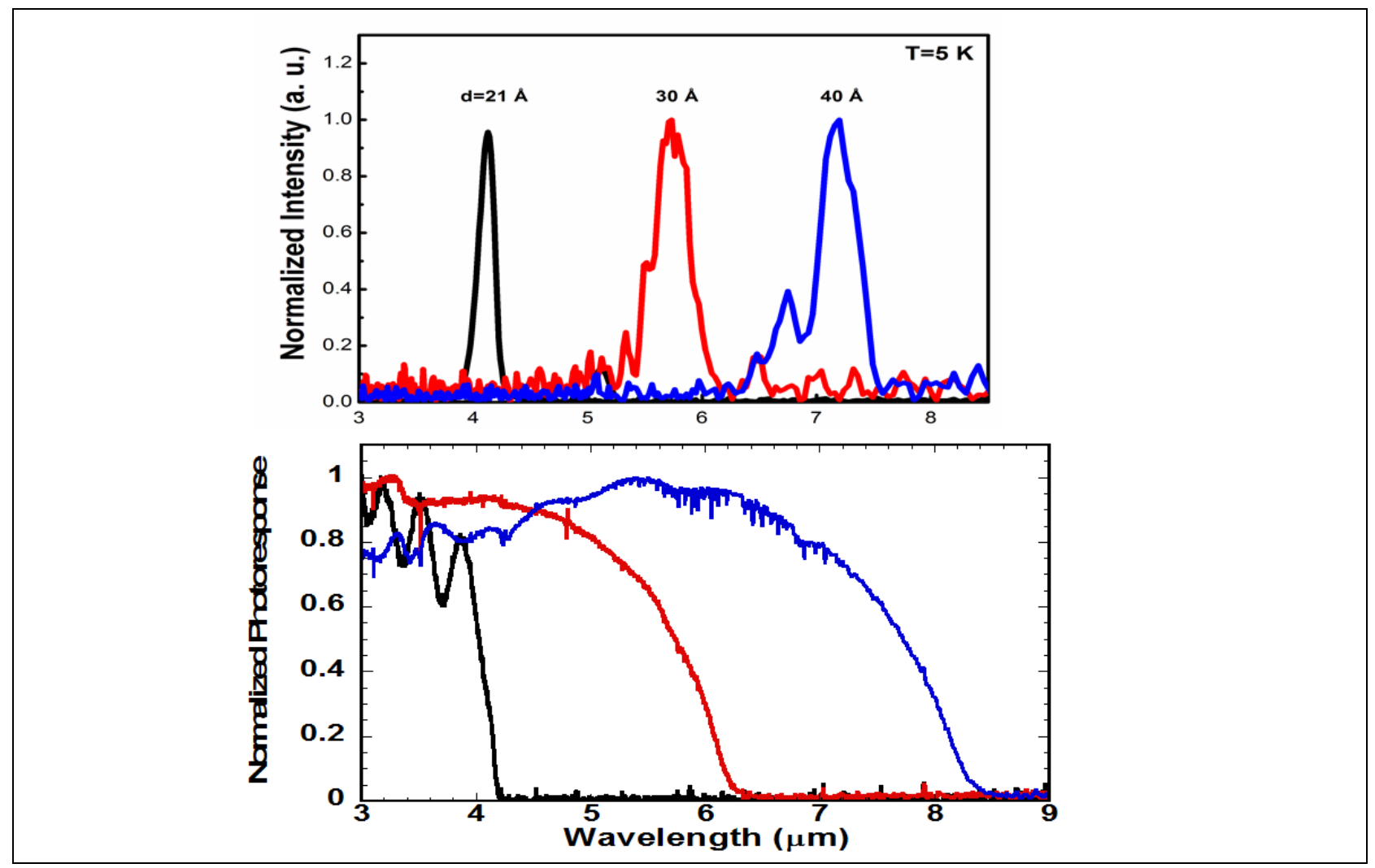

\section{Figure 12. Comparison of the Normalized Photoluminescence and Photo-Conductivity Spectra for the Same Series of Samples where the InAs Width is Varied from 21 to $40 \AA$, while the GaSb Width was kept constant at $26 \AA \AA$.}

The photoresponse spectrum for the sample with an InAs width of 21 does exhibit an odd behavior of strong oscillations superimposed on the spectrum. The period of these oscillations does not match to Fabry-Perot oscillations associated with the superlattice thickness or the total sample thickness including the GaSb substrate. Many other samples with the same design did not have these superimposed oscillations. Since a different $\mathrm{GaSb}$ buffer layer growth procedure was used for this series of samples we wanted to explore if there were any unintended issues with this buffer layer. A SIMS depth profile was performed on a sample from this series. The results of the depth profile are shown in Figure 13. For comparison the depth profile of a similar SL with a different buffer layer growth procedure is included. The SIMS depth profiles of both samples show spikes in oxygen, carbon and silicon at the substrate/buffer layer interface. These spikes are most likely related to polishing and cleaning residuals on the substrate surface. One the other hand, adsorbed surface contamination is common and is typically manifested in such SIMS depth profiles as high surface $\mathrm{C}$ and $\mathrm{O}$ signals that decay steeply, as seen in the $\mathrm{C}$ and $\mathrm{O}$ signals of the left side figure and in the $\mathrm{C}$ of the right side figure. However, the right panel (b)

Distribution Statement A. Approved for public release; distribution unlimited. 
shows an oxygen signal which is higher than that typically observed, and persisting to greater depth. This indicates an increased oxygen concentration in the near-surface region. This subsurface oxygen did have a negative impact on the photoresponse signal.

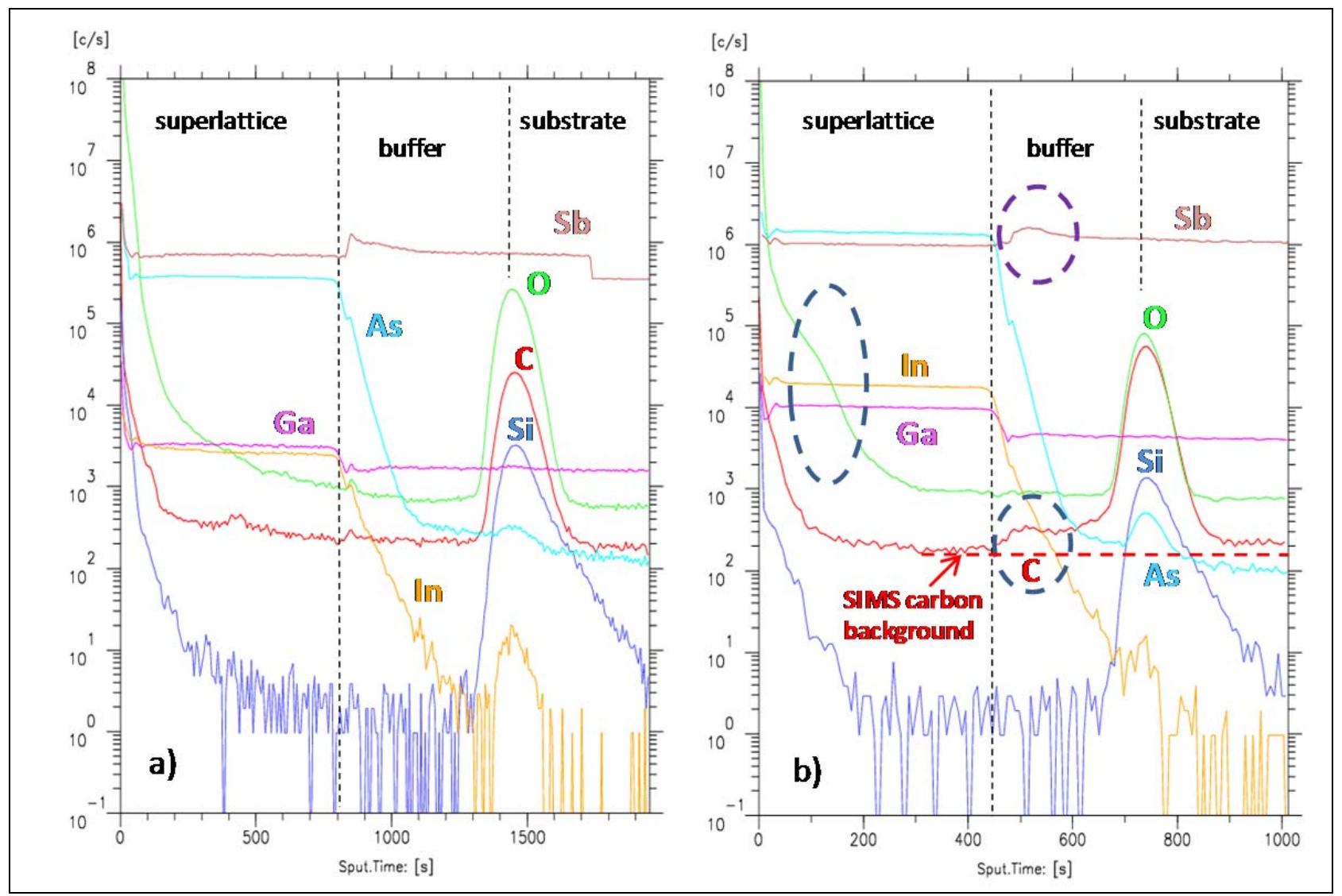

Figure 13. Secondary Ion Mass Spectroscopy Depth Profiles of Two Superlattice Samples with Different Buffer Layer Growth Conditions. Panel a) is our Standard GaSb Buffer Layer Process, b) is the more Gradual Transition from the Buffer Layer Growth Temperature to the Superlattice Growth Temperature. Key Differences are Highlighted by the Dashed Ovals.

The most notable differences between the two depth profiles is that the carbon level in the GaSb buffer is substantially above the SIMS background level and extends closer to the start of the superlattice growth. There is a similar bump in the $\mathrm{Sb}$ level as the growth temperature is slowly ramped down from $490{ }^{\circ} \mathrm{C}$ to $400{ }^{\circ} \mathrm{C}$. So, while the resulting buffer layer surface maybe smoother with the slower decrease in growth temperature there may be more impurities and excess antimony created just under the SL. ${ }^{10}$

In the second series of samples the photoresponse spectra exhibit band gaps from $217 \mathrm{meV}$ to 80

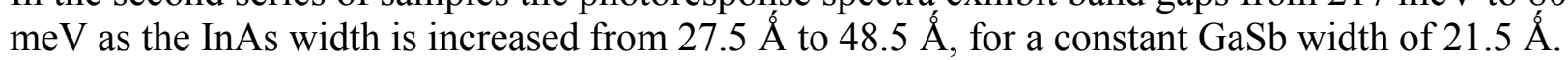
The photoresponse (PR) spectra for the four samples in this series are compared in Figure 14. In this figure the photoresponse spectra aren't normalized to highlight the significant changes in intensity that occur as the SL design is adjusted. To better explore the trend of decreased signal with increasing InAs, the PR intensity, at a point $100 \mathrm{meV}$ above onset of the photoresponse, is

Distribution Statement A. Approved for public release; distribution unlimited. 
plotted versus the corresponding measured band gap energy for each InAs width in Figure 15. The first three high energy data points follow a linear trend. Similar linear trends and slopes have been noted in other sample sets we have studied. What is notable is the rapid drop in signal for the $80 \mathrm{meV}$ sample (48.5 ̊́ InAs).

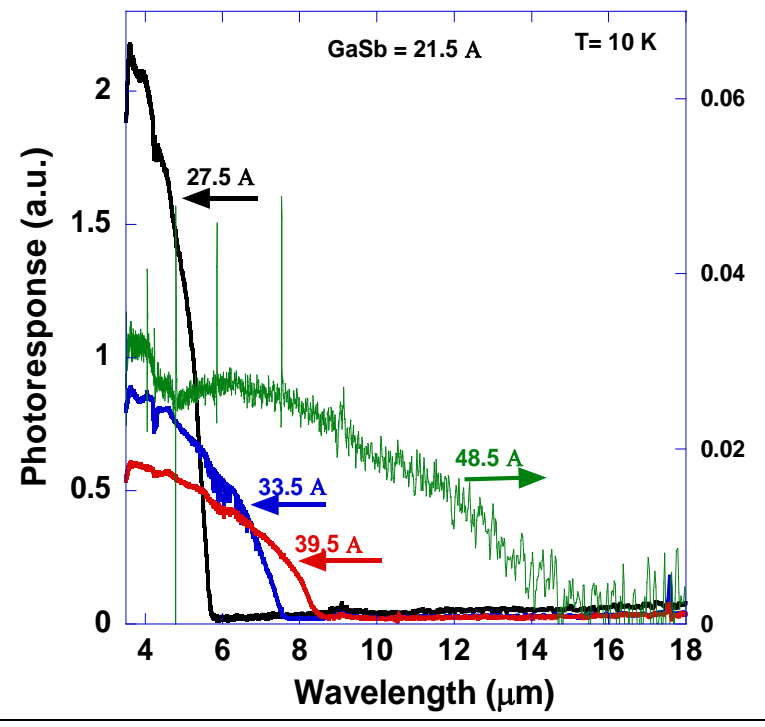

Figure 14. Comparison of the Photoresponse Spectra from 4 Samples with Varying InAs Width. Arrows indicate which Intensity Axis Applies to the Spectra.

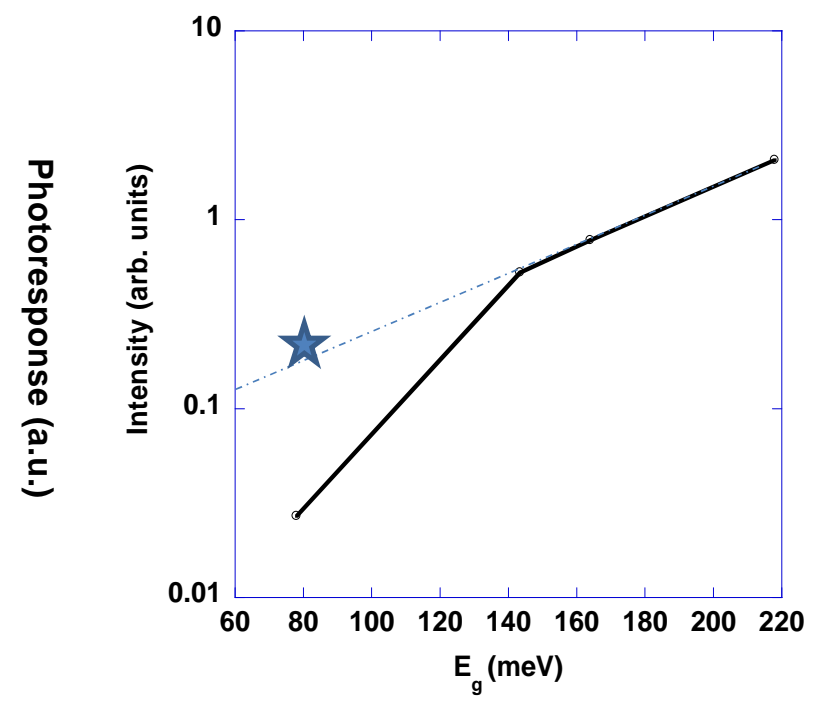

Figure 15. Photoresponse Intensity, $100 \mathrm{meV}$ above the band edge, for spectra shown in Figure 14, as a function of the measured band gap energy. The star marks the results for an improved sample.

One of the optical properties that changes as the InAs width is increased is the oscillator strength, as shown in Figure 16. In type-II superlattices, where the holes and electrons are confined in separate layers, the strength of the optical transition across the SL band gap depends on the degree of overlap of the electron and hole wave functions. As the InAs layer is widened the wave function overlap, and hence oscillator strength, decreases. As the InAs layer is widened

Distribution Statement A. Approved for public release; distribution unlimited. 
from $16 \AA$ to $50 \AA$, with a fixed GaSb width of $24 \AA$, the oscillator strength decreases by a factor of nearly 5 . So even if all the other properties were kept the same, the signal from a VLWIR InAs/GaSb SL would be about 5 times smaller than for a MWIR SL. However, the VLWIR sample was two orders of magnitude weaker. So we need to look for other explanations for this rapid drop in intensity.

There are significant changes in the electrical properties of the superlattices as well when the InAs width is increased. For instance, for a fixed GaSb width of $26 \AA$, the dominant charge carrier in the SL switches from holes to electrons, as the InAs width increases from 30 to $40 \AA$. Hence the MWIR SL designs are typically p-type while the longer wavelength designs are ntype. This change in the majority carrier can have significant impact on SL photodiodes where electrons are the preferred minority carrier. In addition, as the InAs is widened beyond $40 \AA$ Á, the sheet carrier density of the electrons increases. Sheet carrier densities, measured by Hall Effect at $10 \mathrm{~K}$, as a function of InAs width are shown in Figure $17 .^{23}$ Knowledge of the background sheet carrier densities of various SL design choices can be important when choosing the compensating acceptor doping level necessary to make the electrons the minority carrier in the active region of a photodiode. In addition, the samples with the poorest electrical performance are those designs closest to where the dominant carrier type switches. Sample properties rapidly improve with SL designs to either side of this switch in dominant carrier type.

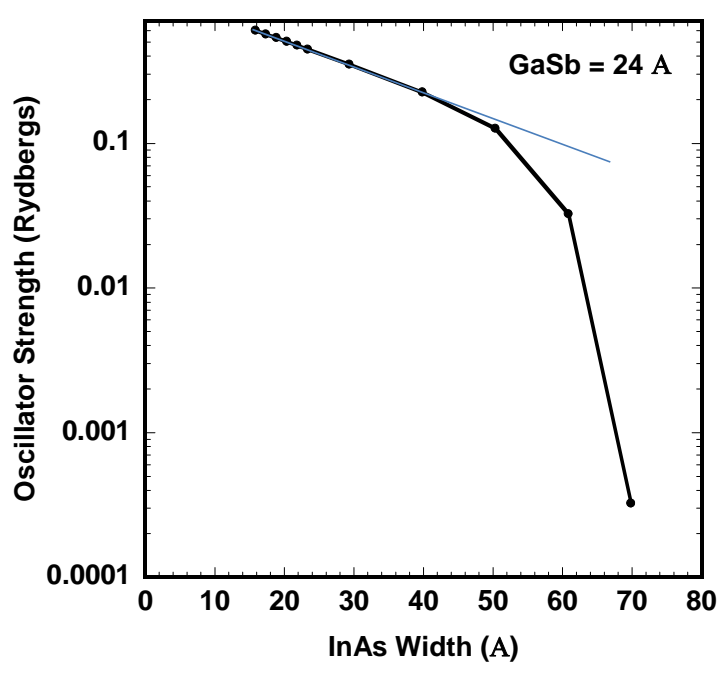

Figure 16. Calculated Oscillator Strength of the Optical Transition across the Superlattice Band Gap as a Function of InAs Width.

Distribution Statement A. Approved for public release; distribution unlimited. 


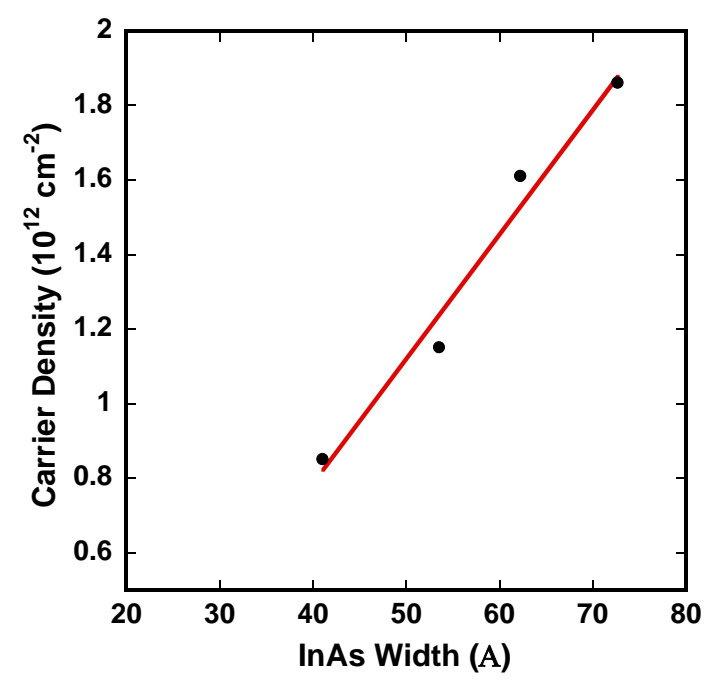

Figure 17. Sheet Carrier Density at 10K Measured by Hall Effect for a SLs with varied InAs Width and Fixed GaSb Width of 24 Á.

Samples with higher conductivity make it more difficult to measure the change in conductivity due to the photon flux in the photoconductivity measurements. But the change in sheet carrier density as InAs is increased from $40 \AA$ to $50 \AA$ is relatively minor so this is not the cause for the two orders of magnitude drop in the photoresponse intensity. Another consideration is residual strain in the SL samples. In this series of samples the interface treatment was not adjusted as the InAs width was changed. So while the MWIR design $(27.5 \AA \AA$ InAs/21.5 $\AA$ GaSb) was strain balanced, the net strain in the other SLs increases. One of the tests performed to check on the superlattice growth results is high resolution x-ray diffraction. This provides rapid feedback on the SL period and residual strain in the SL. The HRXRD rocking curve for the wide period SL design is shown in Figure 18. As expected the measured SL period is very close to the intended SL period, $69 \AA$ Á versus $68.5 \AA$, and within the experimental error of the measurement of $\pm 0.5 \AA$. The large number of superlattice satellite peaks reflects the overall high quality of the sample. The separation of the GaSb substrate peak and the zeroth order SL peak reveals a net strain of $0.56 \%$ in this SL.

Distribution Statement A. Approved for public release; distribution unlimited. 


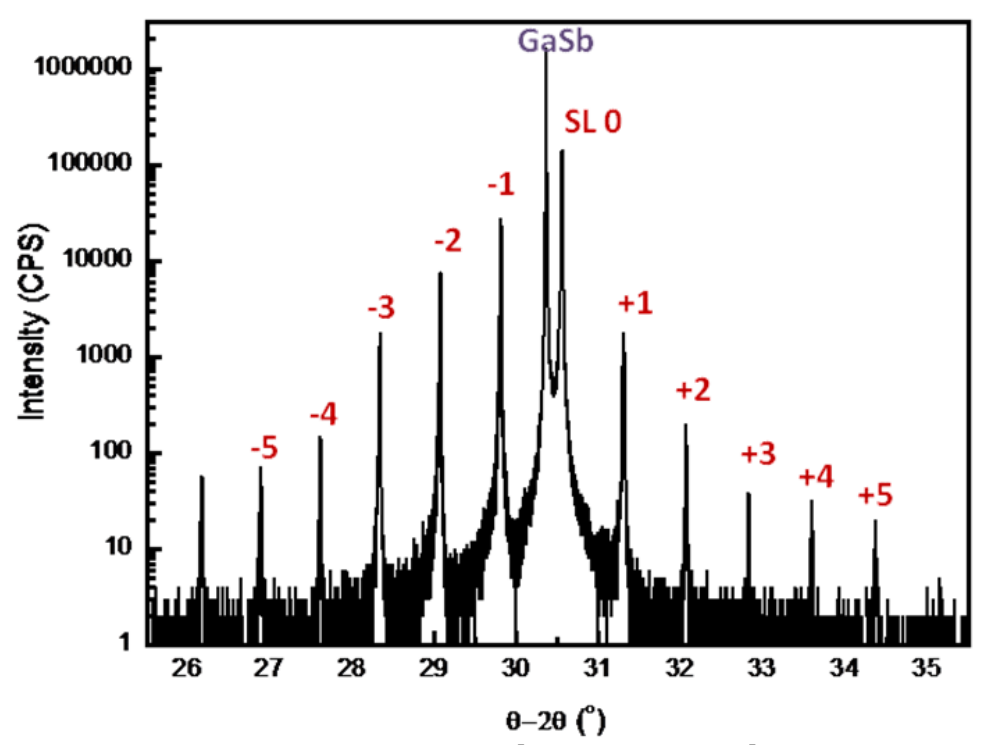

Figure 18. X-ray Rocking Curve for InAs (48 $\AA$ )/GaSb (21 $\AA$ ) Superlattice with a Measured Period of 68.5 $\AA$ and Lattice Mismatch Strain of $\mathbf{- 0 . 5 6 \%}$.

While the HRXRD rocking curve identifies the superlattice period, the individual layer widths and interfaces are not easily determined without extensive simulations. Often we employ high resolution cross-sectional transmission electron microscopy (HRTEM) to examine the individual layer thicknesses and interfaces. Figure 19 is a high resolution cross-sectional TEM image of one of our superlattices, showing the individual layers in the vicinity of the substrate. The sample was designed with an InAs width of $48 \AA$ and a GaSb width of $21 \AA$, and a 6 sec growth interruption was employed at each interface. This image was acquired using a Titan 80-300 TEM, equipped with a spherical aberration (image) corrector, operated at $300 \mathrm{kV}$.

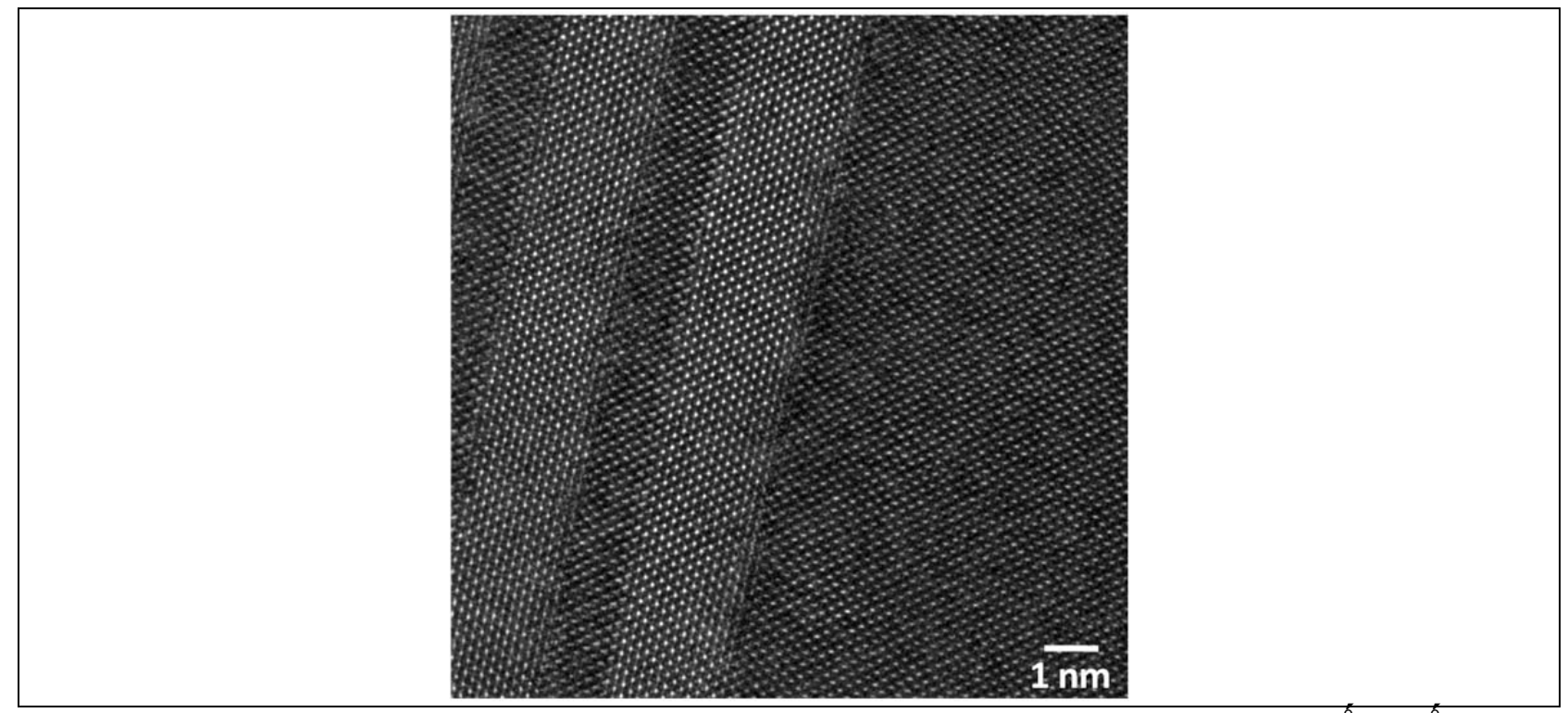

Figure 19. Cross-sectional High Resolution TEM Image of InAs/GaSb $(48 \AA / 20 \AA)$ Superlattice Showing the First Two Periods in the Vicinity of the GaSb (100) Substrate.

Distribution Statement A. Approved for public release; distribution unlimited. 
In addition to imaging the structure of the superlattice layers, methods can be employed for strain determination from HRTEM images. For examination of the strain states in the above superlattice sample we used the recently developed peak-pair analysis method, which is based on analysis in real space. ${ }^{24}$ For the strain analysis, the strain component $\varepsilon_{\mathrm{xx}}$ was parallel to the interface (along [011]) and strain component $\varepsilon_{\mathrm{yy}}$ was along the growth direction ([100]). The strain mapping in the growth direction highlighted the difference in the strain at the InAs-on$\mathrm{GaSb}$ interface versus the GaSb-on-InAs interface. ${ }^{25}$ It was also noted that within the InAs layers, the strain component $\varepsilon_{y y}$ is consistently slightly tensile $(\approx-1 \%)$, which agreed with theoretical calculations of $\varepsilon_{\mathrm{yy}}$ based on published values of elastic constants. An examination of the profiles of $\varepsilon_{\mathrm{xx}}$ showed negligible values, indicating that the interfaces are coherently strained to the GaSb substrate.

The state of strain in the superlattices can have a major impact on the photoresponse of the superlattices. Adjusting the interface growth process and composition can reduce the overall strain in the superlattice structure. One such improved interface growth process was applied to the longer wavelength superlattice design of $48 \mathrm{~A}$ InAs and $21 \mathrm{~A} \mathrm{GaSb}$. A comparison of the photoresponse spectra for two nearly identical SLs with only the interface process improved is shown in Figure 20. By adjusting the interfaces, the photoresponse intensity was improved by nearly two orders of magnitude. This improved photoresponse intensity is plotted as the star data point in Figure 15. Now the photoresponse intensity for the narrow band gap design falls near the dashed trend line of the other samples.

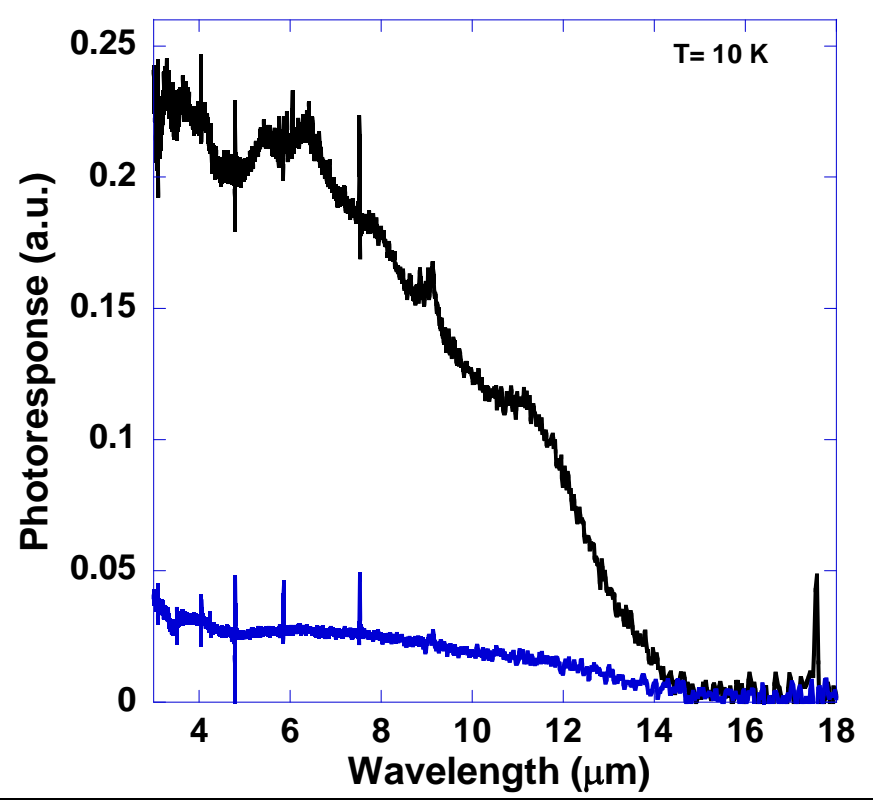

Figure 20. Comparison of the Photoresponse Spectra of Two InAs (21 $\AA$ )/GaSb (48 $\AA$ ) Superlattice Samples with Different Interface Processes. The weaker spectrum is the same as shown in Figure 14.

Distribution Statement A. Approved for public release; distribution unlimited. 


\section{SUMMARY}

Adjusting the design of type-II superlattices can lead to many other changes in the material besides the expected energy band structure and hence band gap. There are complex trade-offs in the background carrier concentration, the dominant charge carrier type, net strain, absorption strength, carrier mobility and carrier lifetime that occur. Fundamental measurements on basic superlattice materials can provide insight into what electrical, optical and structural parameters are changing even when a change as small as a $10 \AA$ increase in a layer thickness is made. The combination of theoretical modeling, systematic MBE studies, and advanced characterization techniques, in the Materials \& Manufacturing Directorate of the Air Force Research Laboratory, greatly enhances the ability to understand the origin of these property changes and lead to further improvements for future infrared detectors based on type-II superlattices.

\section{REFERENCES}

[1] D. L. Smith and C. Mailhiot, J. Appl. Phys. 62, 2545 (1987).

[2] G. A. Sai-Halasz, R. Tsu and L. Esaki, Appl. Phys. Lett. 30, 651 (1977).

[3] C.-H. Lin, G. J. Brown, W. C. Mitchel, M. Ahoujja and F. Szmulowicz, SPIE Proc. 3287, 22 (1998).

[4] F. Szmulowicz, E. R. Heller, K. Fisher, and F. L. Madarasz, Superlattices and Microstructures 17, 373 (1995).

[5] J. L. Johnson, L. A. Samoska, A. C. Gossard, J. L. Merz, M. D. Jack, G. R. Chapman, B. A. Baumgratz, K. Kosai and S.M. Johnson, J. Appl. Phys. 80, 1116 (1996).

[6] J. B. Rodriguez, P. Christol, F. Chevrier and A. Joullié, Physica E 28, 128 (2005).

[7] J.V. Li, C. J. Hill, J. Mumolo, S. Gunapala, S. Mou, and S-L. Chuang, Appl. Phys. Lett. 93, 163505 (2008).

[8] C. Canedy, E. Aifer, J. Warner, I. Vurgaftman, E. Jackson, J. Tischler, S. Powell, K. Olver, J. Meyer and w. E. Tennant, Infrared Phys. \& Technol. 52, 326 (2009).

[9] E. K-w. Huang, D. Hoffman, B-M. Nguyen, P-Y. Delaunay and M. Razeghi, Appl. Phys. Lett. 94, 053506 (2009).

[10] H. J. Haugan, S. Elhamri, W. C. Mitchel, B. Ullrich, G. J. Brown, L. Grazulis and S. Houston, SPIE Proc. 7222, 72220Y (2009).

[11] R. Rehm, M. Walther, J. Schmitz, F. Rutz, J. Fleibner, R. Scheibner and J. Ziegler, Proc. SPIE 7222, 72220T (2009).

[12] A. Rogalski, SPIE Proc. 5834, 1 (2005).

[13] C. H. Grein, P. M. Young, M. E. Flatté and H. Ehrenreich, J. Appl. Phys. 78, 7143 (1995).

[14] H. J. Haugan, G. J. Brown, F. Szmulowicz, L. Grazulis, W. C. Mitchel, S. Elhamri, and W. D. Mitchell, J. Crystal Growth 278, 198 (2005).

[15] K. Mahalingam, K. G. Eyink, G. J. Brown, D. L. Dorsey, C. F. Kisielowski and A. Thust, Appl. Phys. Lett. 88, 091904 (2006).

[16] H. J. Haugan, S. Elhamri, F. Szmulowicz, B. Ullrich, G. J. Brown and W. C. Mitchel, Appl. Phys. Lett. 92, 071102 (2008).

[17] H. J. Haugan, S. Elhamri, G. J. Brown and W. C. Mitchel, J. Appl. Phys. 104, 073111 (2008).

Distribution Statement A. Approved for public release; distribution unlimited. 
[18] H. J. Haugan, S. Elhamri, B. Ullrich, G. J. Brown, and W. C. Mitchel, J. of Crystal Growth 311, 1897 (2009).

[19] C. Mailhiot and D. L. Smith, J. Vac. Sci. Technol. B 5, 1268 (1987).

[20] Y. Wei, M. Razeghi, and G. J. Brown, SPIE Proc. 5359, 301 (2004).

[21] G. J. Brown, H. J. Haugan, F. Szmulowicz, K. Mahalingam, S. R. Munshi, B. Ullrich and S. Houston, SPIE Proc. 6127, 61271I (2006).

[22] B. Ullrich, A. Davis (to be published).

[23] F. Szmulowicz, S. Elhamri, H. J. Haugan, G. J. Brown and W. C. Mitchel, J. Appl. Phys. 101, 043706 (2007).

[24] P.L. Galindo, S. Kret, A. M. Sanchez, J-Y. Laval, A. Yanez, J. Pizarro, E. Guerrero, T. Ben, and S. I. Molina, Ultramicroscopy 107, 1186 (2007).

[25] K. Mahalingam, H. J. Haugan, G. J. Brown, K. G. Eyink, F. Szmulowicz, B. Jiang and C. F. Kisielowski, SPIE Proc. 7608, 76081S (2010).

Distribution Statement A. Approved for public release; distribution unlimited. 


\title{
2.4 Phase Sensitive Detection of Photoluminescence with Fourier Transform Spectroscopy
}

B. Ullrich and G. J. Brown

Air Force Research Laboratory, Materials and Manufacturing Directorate, Wright Patterson AFB, Ohio 45433-7707, USA

\begin{abstract}
Although this technique has been used for more than twenty years, there is scant information in the literature regarding the experimental parameters of double modulation Fourier transform infrared photoluminescence. Measuring the emission of InSb:Te, we present the elucidation of optimized parameters using the combination of a Fourier transform spectrometer and lock-in amplifier.
\end{abstract}

Photoluminescence (PL) measurements of semiconductors in the mid- and far infrared energy range (specifically towards $100 \mathrm{meV}$ and below) encounter serious difficulties due to the thermal background radiation, which alters or even masks the actual PL spectrum. ${ }^{1-4}$ In order to suppress black body radiation, two double modulation techniques - both utilizing a Fourier transform spectrometer - have been employed. Rowell and Buijs ${ }^{1}$ used phase sensitive detection (PSD), while Fuchs et al. ${ }^{2}$ promoted phase sensitive excitation (PSE). In both papers the BOMEM DA3 spectrometer was employed. This Fourier transform infrared (FTIR) spectrometer consists basically of an interferometer (beamsplitter, a fixed mirror, and a moving mirror) and a detector. By exciting the sample with a light source above the band gap and guiding the PL signal into the interferometer, an interferogram that has all the infrared frequencies "encoded" is recorded. ${ }^{5}$ In order to obtain the emission spectrum, the signal is translated from the time domain to the frequency (energy) domain by Fourier transformation. Consequently, it is crucial to FTIR experiments to know exactly the position of the moving mirror at any given moment. The instrument's resolution is dependent on the path length of the mirror, i.e. the distance moved during a scan, while the bandwidth is determined by the sampling rate. For high resolution a long mirror path is required and for wide bandwidth the precise measurement of the mirror position is necessary. ${ }^{6}$ For the control of the sampling rate of the interferogram the BOMEM is equipped with He-Ne laser. This dynamic calibration mechanism during scanning due to the generated harmonic modulation of the reference laser beam refers to Connes Advantage ${ }^{5,6}$ with respect to dispersive instruments. The benefit of internal calibration is accuracy and precision of better than $0.1 \mathrm{~cm}^{-1}$ by correcting mechanically caused errors. The PSE method uses the modulated He-Ne laser emission to create an amplitude modulation of the exciting light source in phase with the interferometer. ${ }^{2-4}$ As described in detail in Ref. 2, this method delivers the PL spectrum as upper and lower sidebands of the He-Ne laser frequency. The PSD method, on the other hand, employs lock-in technique by chopping the impinging light beam with an external chopper, i.e., the modulation frequency of the exciting light source and the internally generated frequency are decoupled and the PL spectrum is not a related sideband of the He-Ne laser emission. ${ }^{1,7}$ Notably, although around for more than twenty years, the influence of the experimental parameters on FTIR spectroscopy and their optimization was scarcely discussed or completely omitted in the literature. In a previous work we used PSD for the PL measurements of InAs/GaSb superlattices. ${ }^{8}$ In this paper, we discuss the proper PSD conditions for FTIR measurements by demonstrating the influence of the lock-in parameters (low pass filter settings and dynamic reserve) and the scan speed of the spectrometer.

Distribution Statement A. Approved for public release; distribution unlimited. 
As in Refs. 1-4, the BOMEM DA3 equipped with a He-Ne laser was used for the measurements. The optical excitation of the sample was performed with the $532 \mathrm{~nm}$ constant wavelength (cw) emission of a $2 \mathrm{~W}$ Coherent Verdi laser. The impinging intensity on the sample, which was mounted in a closed cycle He gas cryostat manufactured Janis, was about $60 \mathrm{~W} / \mathrm{cm}^{2}$. The spectra were recorded by using a $\mathrm{KBr}$ beamsplitter and a nitrogen cooled MCT detector. The lock-in used was the model SR830 from Stanford Research Systems (SRS) and the reference frequency was provided by a high speed optical chopper from HINDS Instruments chopping the laser beam at $60 \mathrm{kHz}$. The sample used was a commercial $n$-type InSb:Te wafer. All measurements were carried out at $4.7 \mathrm{~K}$.

Following the basic description of Rowell and Buijs ${ }^{1}$, the PL was measured by routing the output of the MCT detector to the lock-in amplifier. The $x$-channel output of the lock-in was then fed back into the analogue input of the BOMEM spectrometer. Probably the most critical PSD parameter is the correct choice of the lock-in output time constant $\tau$ of the low pass filter. We stress that lock-in amplifiers are synchronous rectifiers. Hence, the output of the lock-in is supposed to be a direct current (DC) signal. However, noise from the input is transferred to the output, requiring smoothing of the actual output signal. ${ }^{9}$ Straight forwardly, $\tau$ can be interpreted as "reaction time" of the lock-in amplifier. As seen below, for the current application, compared to dispersive spectroscopy, the $\tau$ values might by three orders of magnitude smaller, i.e., in the $\mu$ s range.

In order to prevent extrinsic distortion of the interferogram, it is required that the external chopping frequency $f_{\text {ch }}$ exceeds considerably the internal sampling rate of the BOMEM and, in-at the same time, that $\tau$ is shorter than the sampling time, which is given by,

$$
t_{\mathrm{s}}=1 /\left(2 m v k^{\prime}\right)
$$

where, $m$ is the sampling rate per He-Ne laser fringe (commonly, as in our experiments, $m=1$ ), $v$ is the scanning speed of the mirror and $k^{\prime}$ is the spectroscopic wavenumber of the He-Ne emission defined by one over wavelength $(1 / \lambda)$, using cgs units $\left(15798 \mathrm{~cm}^{-1}\right)$. The factor 2 in Equation (16) considers the light travel up and down of the moving mirror. With $k^{\prime}=15798 \mathrm{~cm}^{-1}$ and a typical scan speed of $0.5 \mathrm{~cm} / \mathrm{s}, t_{\mathrm{s}}=63.3 \mu \mathrm{s}$. This result shows that high speed optical choppers are indeed required in order to keep the desired one-order-of-magnitude difference (or more) between the sampling frequency and $f_{\text {ch }}$. By slowing down the scan speed $(\leq 0.05 \mathrm{~cm} / \mathrm{s})$ the requirement $f_{\mathrm{ch}}>>1 / t_{\mathrm{s}}$ is easily satisfied with $f_{\mathrm{ch}}=60 \mathrm{kHz}$. Equivalently, regarding the modulated PL signal, it is required that $f_{\mathrm{ch}} \gg f_{\mathrm{PL}}$, where $f_{\mathrm{PL}}$ is the samples' anticipated PL frequency. ${ }^{10}$ The latter is expressed by $f_{\mathrm{PL}}=2 v / \lambda_{\mathrm{PL}}$, where $\lambda_{\mathrm{PL}}$ is the emission wavelength (around $4 \mu \mathrm{m}$ for InSb). Additionally, the requirement $f_{\mathrm{ch}}>>1 / \tau$, where $\tau$ is the lock-in output time constant of the low pass filter, has to be satisfied. Based on these considerations and systematic experiments using various parameters, as shown below, resulted in the requirements for FTIR PSD:

$$
0.1 \times f_{\mathrm{ch}} \geq 1 / t_{\mathrm{s}} \leq 0.2 / \tau
$$

and,

$$
0.1 \times f_{\mathrm{ch}} \geq 1 / \tau \geq 10 \times f_{\mathrm{PL}}
$$

Distribution Statement A. Approved for public release; distribution unlimited. 
The spectra (a) and (c) in Figure 21 show the result of PSD in comparison with the standard measurement without a lock-in amplifier. The figure demonstrates the impact of the double modulation technique - the black body radiation considerably alters the PL of the InSb sample. We note that the spectral shape of the PL shows similarities to the emission intrinsic $\mathrm{InSb}^{7}$ but $^{11}$ the PL spectra in Figure 21 are shifted towards higher energies as expected for $n$-type InSb. ${ }^{11}$ The experimental conditions ( $v=0.02 \mathrm{~cm} / \mathrm{s}$ and $\tau=300 \mu \mathrm{s}$ ) result in $t_{\mathrm{s}}=1.6 \mathrm{~ms}, 1 / \tau=3.3 \mathrm{kHz}$, and $f_{\mathrm{PL}}=100 \mathrm{~Hz}$. Hence, the requirements (2) $[6 \mathrm{kHz}>625 \mathrm{~Hz} \leq 667 \mathrm{~Hz}]$ and (3) $[6 \mathrm{kHz} \geq 3 \mathrm{kHz}>1 \mathrm{kHz}]$ are satisfied. The results also demonstrate that 1000 scans [spectrum (c)] produces a zero line with a similar low noise level as the standard measurement [spectrum (b)]. The perfection of the zero line is our measure for identifying the appropriate choice of the selected parameters. The somewhat increased noise level in the range $0.35-0.45 \mathrm{eV}$ is caused by a harmonic of the excitation laser.

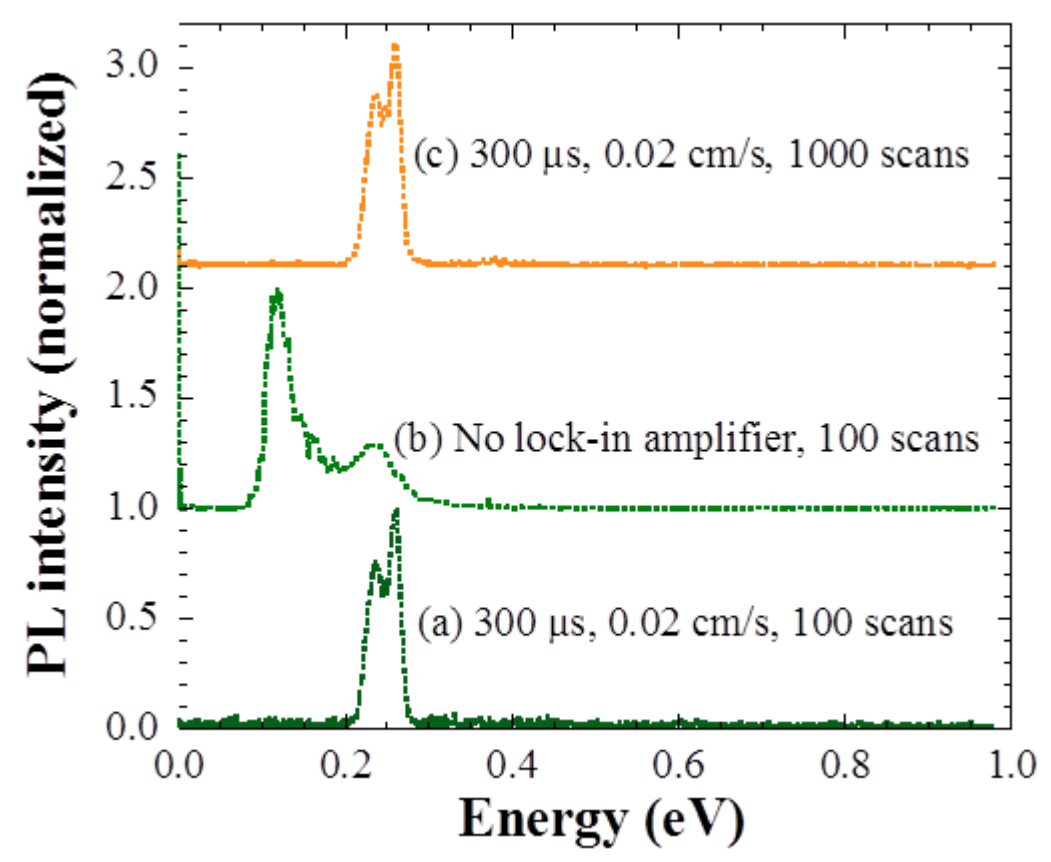

Figure 21: Comparison of various InSb:Te PL spectra : (a) PL intensity measured with PSD, (b) PL intensity measured without lock-in amplifier, and (c) shows the same measurement as spectrum (a) but with 1000 scans rather than 100 scans. Indicated are the parameters - BOMEM scan speed, lock-in time constant, and scan speed - used for the measurements. Spectrum (b) is recorded with the default scanning speed of $0.5 \mathrm{~cm} / \mathrm{s}$.

Further proof of validity of requirements (2) and (3) is demonstrated in Figure 22, which shows PL spectra (all measured with $v=0.02 \mathrm{~cm} / \mathrm{s}$ ) for different time constant and roll off filter settings: (a) $300 \mu \mathrm{s}, 24 \mathrm{~dB}$, (b) $300 \mu \mathrm{s}, 12 \mathrm{~dB}$, (c) $1 \mathrm{~ms}, 12 \mathrm{~dB}$, and (d) $3 \mathrm{~ms}, 12 \mathrm{~dB}$. Spectra (a) and (b) reveal satisfactory results, while the increase to $1 \mathrm{~ms}$ and $3 \mathrm{~ms}$ produced artificial features particularly in the low energy side of the spectrum. It is readily shown that the lock-in settings of spectrum (c) and (d) violate requirement (2), while requirement (3) does not concur only with the settings of spectrum (d). The results further stress that the filter roll off ( $12 \mathrm{~dB}$ vs. $24 \mathrm{~dB})$ does not considerably impact the overall appearance the spectra. Figure 23 demonstrates the sensitivity of the PL response on the BOMEM scan speed. The increase from $0.02 \mathrm{~cm} / \mathrm{s}$

Distribution Statement A. Approved for public release; distribution unlimited. 
[spectrum (a)] to $0.05 \mathrm{~cm} / \mathrm{s}$ [spectrum (b)] spoils notably the zero line by producing artificial features in the vicinity of the actual PL spectrum due to the violation of requirement (2).

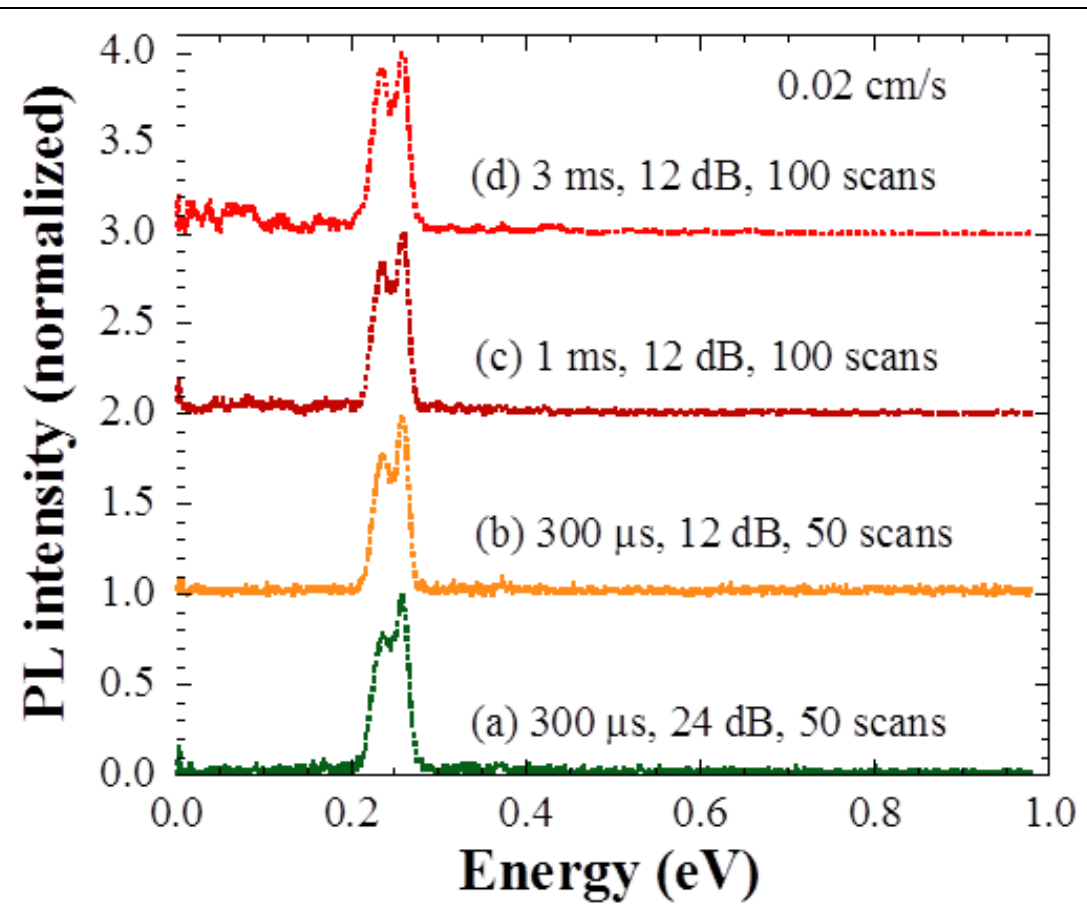

Figure 22: Comparison of InSb:Te PL spectra recorded with PSD at various lock-in time constant and roll off settings: (a) $300 \mu \mathrm{s}, 24 \mathrm{~dB}$, (b) $300 \mu \mathrm{s}, 12 \mathrm{~dB}$, (c) $1 \mathrm{~ms}, 12 \mathrm{~dB}$, and (d) 3 $\mathrm{ms}, 12 \mathrm{~dB}$. All spectra were recorded with a BOMEM scan speed of $0.02 \mathrm{~cm} / \mathrm{s}$.

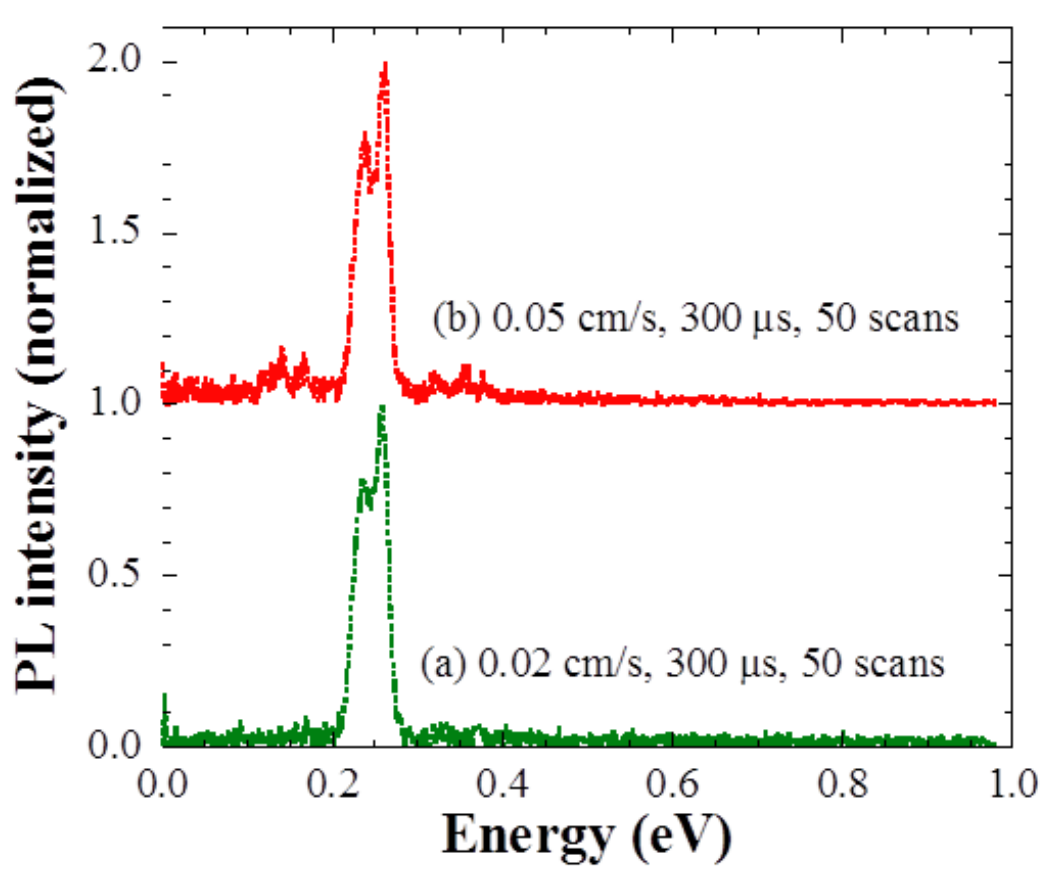

Figure 23: Demonstration of the impact of the BOMEM scan speed variation on the PSD of the InSb:Te emission: (a) $0.02 \mathrm{~cm} / \mathrm{s}$ and (b) $0.05 \mathrm{~cm} / \mathrm{s}$.

Distribution Statement A. Approved for public release; distribution unlimited. 
The consequence of violating requirement (3) is revealed in Figure 24, which shows a detailed comparison of the PL spectrum measured at $\tau=100 \mu$ s (violating requirement (3) only) with spectra recorded at $300 \mu \mathrm{s}, 1 \mathrm{~ms}$, and $3 \mathrm{~ms}$, i.e., spectrum (a) in Figure 21, and spectra (c) and (d) in Figure 22, respectively. At shorter time constants the spectral features reveal improved contrast but at an increased noise level. Amplified noise at shorter time constants is an intrinsic part of the lock-in concept and therefore not a surprise per se but the PL intensity decrease at 100 $\mu$ indicates that $f_{\text {ch }}(=60 \mathrm{kHz})$ and $1 / \tau(=10 \mathrm{kHz})$ were not sufficiently separated any more. The point from where on the signal amplitude stagnates or decreases when the time constant is shortened (i.e., at $\tau=300 \mu$ s in Figure 24) refers to the break-even point between lock-in parameters and BOMEM settings, resulting in PL spectra with optimized signal-to-noise ratios.

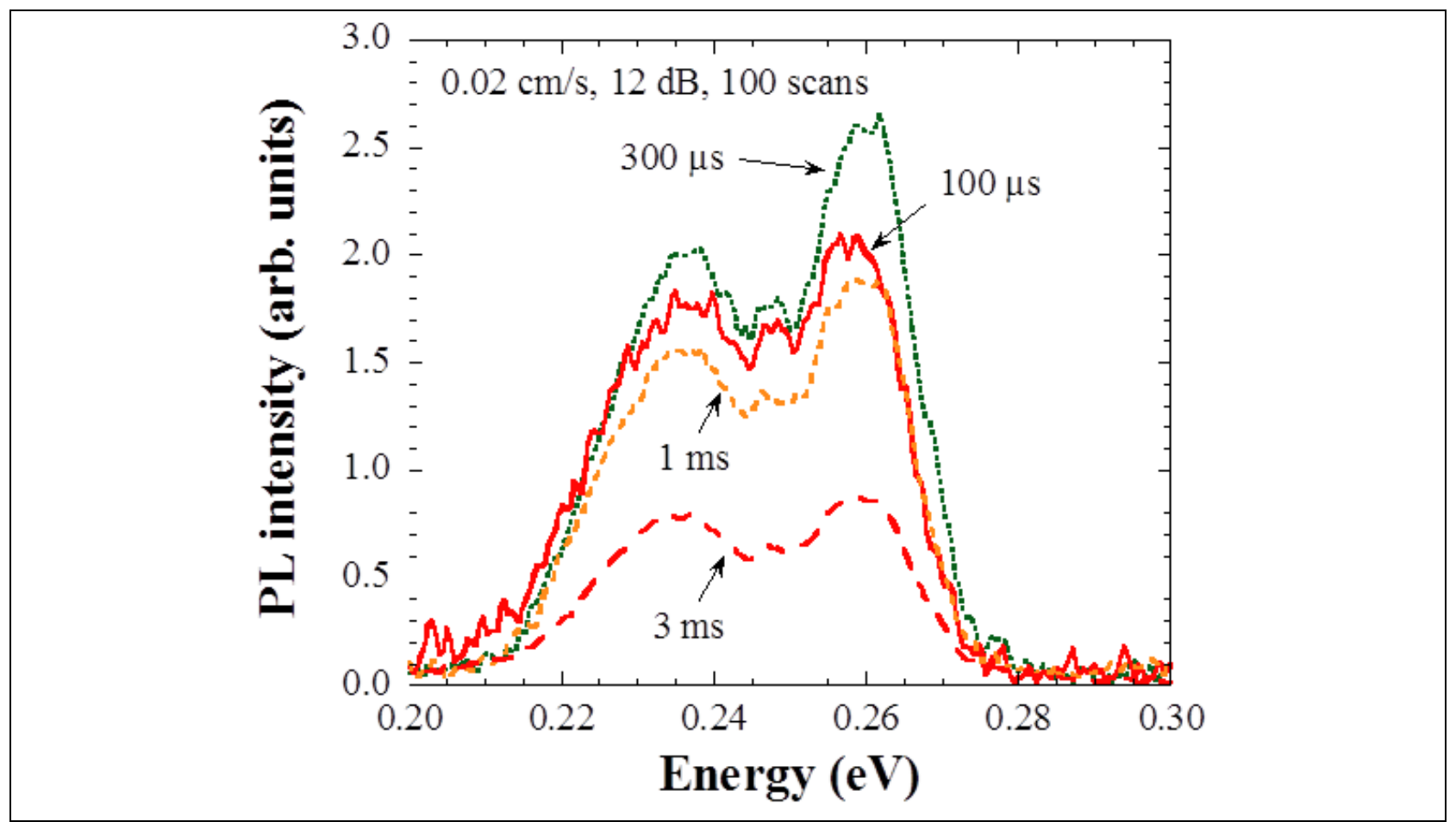

\section{Figure 24: InSb:Te PL spectra measured with PSD using various lock-in time constants. As indicated, besides the time constant, the remaining parameters were the same for all spectra.}

A further lock-in parameter of importance is the dynamic reserve. The SR830 lock-in has three choices - low noise, normal and high reserve. It turned out that the selection high reserve gave slightly better signal-to-noise ratios than experiments carried out with the setting normal. Measurements performed with low noise however produced results similar to spectrum (d) in Figure 22. Hence, the majority of our experiments were performed with the setting high reserve, taking full benefit of the advanced digital demodulation technique of the used lock-in model.

To summarize, PSD requires "slow" scan speeds $(\leq 0.05 \mathrm{~cm} / \mathrm{s})$ and rather "short" time constant settings (upper $\mu$ s to lower ms range). Chopping at $60 \mathrm{kHz}$ and employing the BOMEM DA3 in conjunction with the SR830 lock-in amplifier, the optimized measuring conditions for infrared

Distribution Statement A. Approved for public release; distribution unlimited. 
PL are $v=0.02 \mathrm{~cm} / \mathrm{s}, \tau=300 \mu \mathrm{s}, 12 \mathrm{~dB}$, and high reserve. These parameters have been elucidated by altering the variables in successive PL experiments on InSb:Te.

\section{References}

$1 \quad$ N. L. Rowell and H. Bujis, Mikrochim. Acta (Vienna) I, 435 (1988).

2 F. Fuchs, A. Lusson, J. Wagner, and P. Koidl, in Proceedings of the Seventh International Conference on Fourier Transform spectroscopy, Fairfax, 1989, SPIE Symposia Proceedings No. 1145 (SPIE Bellingham, WA, 1989), p. 323.

3 F. Fuchs, A. Lusson, P. Koidl, and R. Triboulet, J. Crystal Growth 101, 722 (1990)

4 F. Fuchs, H. Schneider, P. Koidl, K. Schwarz, and H. Walcher, Phys. Rev. Lett. 67, 1310 (1991).

5 http://www.thermo.com/eThermo/CMA/PDFs/Articles/articlesFile_1195.pdf

6 http://boards.straightdope.com/sdmb/archive/index.php/t-345590.html

$7 \quad$ N. L. Rowell, Infrared Physics 28, 37 (1988).

8 H. J. Haugan, B. Ullrich, S. Elhamri, F. Szmulowicz, G. J. Brown, L. C. Tung, and Y. J. Wang, J. Appl. Phys. 107, 083112 (2010).

9 Operating Manual and Programming Reference, SR830, DSP Lock-In Amplifier, Stanford Research Systems, Revision 2.3 (12/2006).

10 K. O'Brien, Mid-infrared Laser Diode Performance and Suppression of Auger Loss, Doctor of Philosophy (PhD), Department of Physics, School of Electronics and Physical Sciences University of Surrey, 2007.

11 A. S. Filipchenko, T. B. Kurenkeev, and T. Seisenbaev, Phys. Stat. Sol. (a) 50, K251 (1978).

Distribution Statement A. Approved for public release; distribution unlimited. 


\subsection{Quantum Dot Research}

\subsection{TEM Characterization of InGaAs QDIP structures}

In this report we present a transmission electron microscope (TEM) study of quantum dot infrared photodetector (QDIP) structures, consisting of InAs quantum dots (QDs) grown on (100) GaAs substrates. The structures examined contained multiple stacks, wherein each QD layer had a 20 ML-thick capping layer of $\operatorname{In}_{0.15} \mathrm{Ga}_{0.85} \mathrm{As}$ which was followed by a $180 \mathrm{ML}$-thick GaAs spacer layer. The main objective of this study was to investigate the structural integrity, specifically the propensity for dislocation generation (and propagation), with increasing number of QD stacks in the structure. Several samples with the number of QDIP stacks ranging from 120 were examined in this study. The diffraction patterns shown in Figure 25 represent the typical conditions used for imaging the QD layers. Specifically, the two-beam diffraction condition for obtaining the bright-field $(\mathrm{G}=200)$ and (200) dark-field images is shown in Figure 25 (a) and that for the bright-field $(\mathrm{G}=022)$ and $(022)$ dark-field images in Figure 25 (b).

(a)

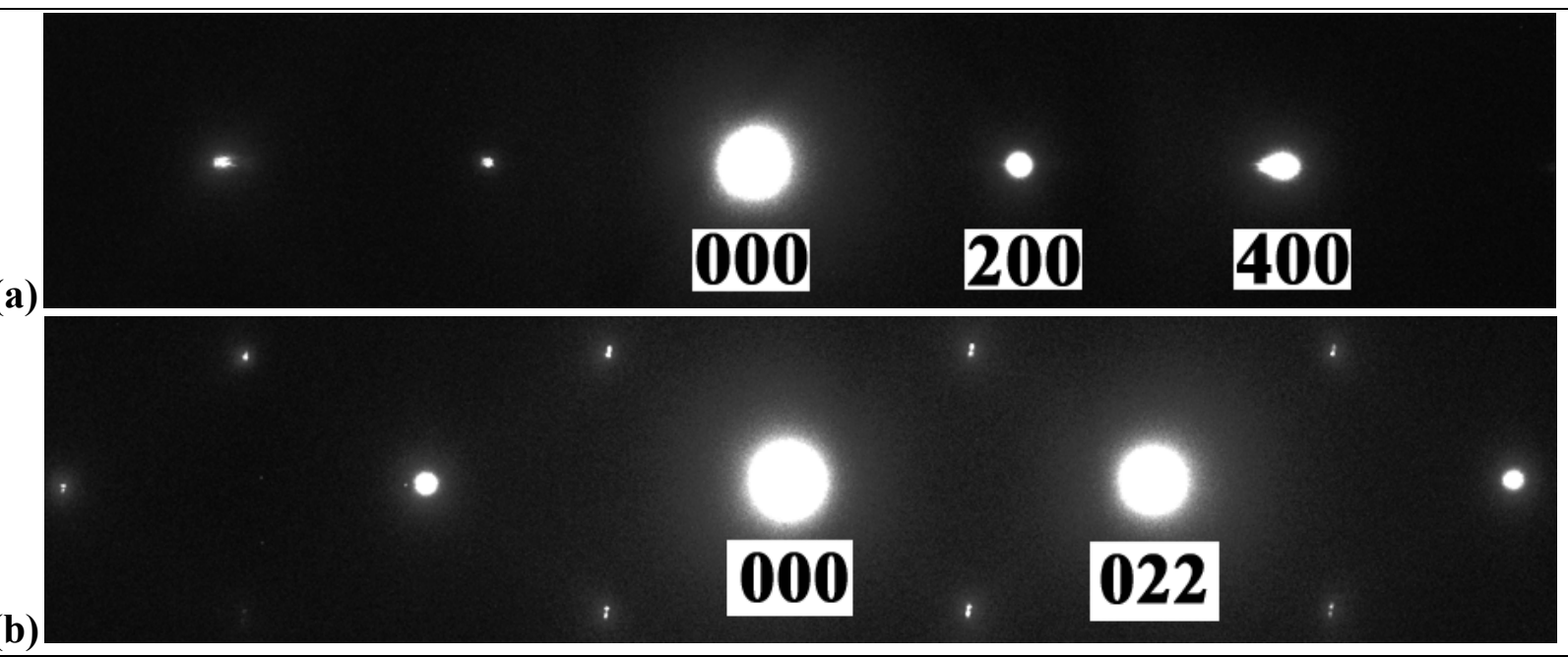

Figure 25. Diffraction patterns representing the two beam imaging conditions used for (a) $G=200$ and (b) $G=022$ bright-field and dark-field images.

Figure 26 (a) is a bright-field $(\mathrm{G}=(022))$ of a single QD layer structure, in which the contrast due to the strain-field associated with the QDs is clearly visible. However, it is evident that no dislocation nucleation occurs in these regions. An examination of the (200) dark-field image, shown in Figure 26 (b), further reveals that the InGaAs layer is of uniform thickness. Figures $27-$ 29 show the bright-field $(\mathrm{G}=(022))$ and $(200)$ dark-field images of samples in which the number of 5, 10 and 20 QD stacks. From a comparison of these images with those shown in Figure 26, it is clear that there is no significant change in structural evolution with increase in number of QD layers. In particular, it is observed that structural integrity of each QD layer is well maintained with no evidence for nucleation/propagation of dislocations. The dislocation density in many of the above samples was evaluated by plan-view TEM. Figure 30 is a (220) dark-field image of the sample with a single QD layer showing a structure in which the QDs exhibit the typical AshbyBrown contrast (i.e. two dark or bright lobes), indicating a high degree of coherency. Figure 31 is a (220) dark-field plan-view TEM image of the sample with 10 QD stacks. In this image the QDs are not visible due to presence of a thicker $(3 \mu \mathrm{m})$ GaAs contact layer. In contrast to the single QD layer sample (Figure 30), the presence of dislocations is clearly observed in this sample. The oscillating contrast exhibited in these images indicates that the dislocations

Distribution Statement A. Approved for public release; distribution unlimited. 
propagate from the bottom to the top surface of the film. Based on an analysis of several images similar to that shown in Figure 7, the dislocation density in this sample is estimated to be about $10^{8} / \mathrm{cm}^{2}$. Noting that the image in Figure 7 and cross-sectional TEM images shown in Figure4 are from the same sample, it is not possible to identify the source of these dislocations.

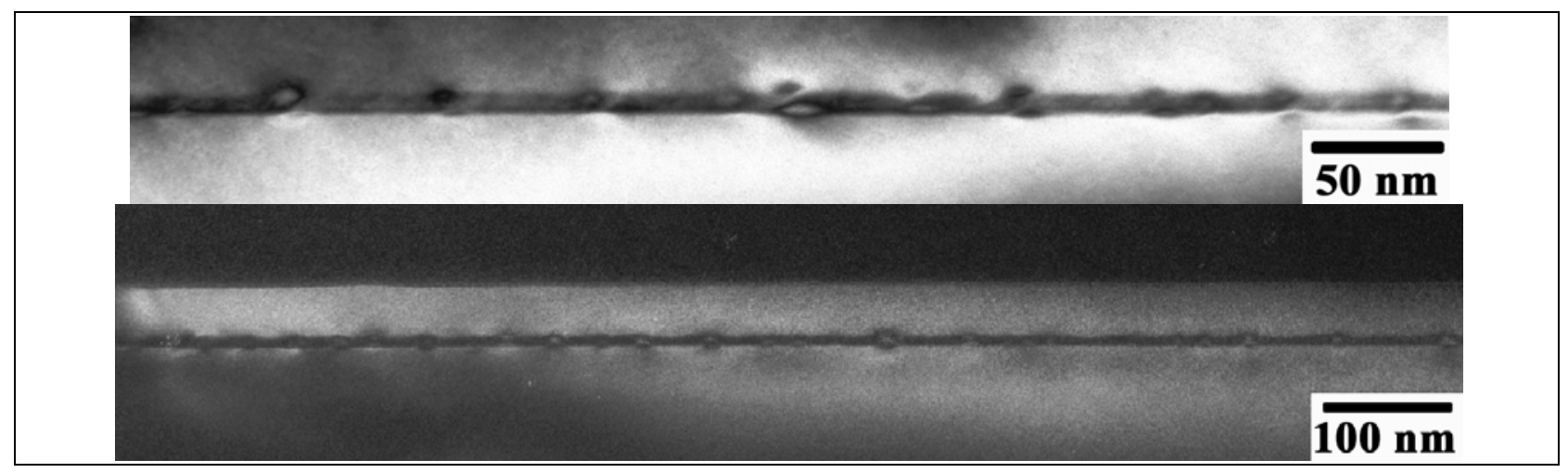

Figure 26. Cross-sectional TEM images of the single-layer QDIP structure showing (a) $G=(022)$ bright-field image and (b) (200) dark-field image.

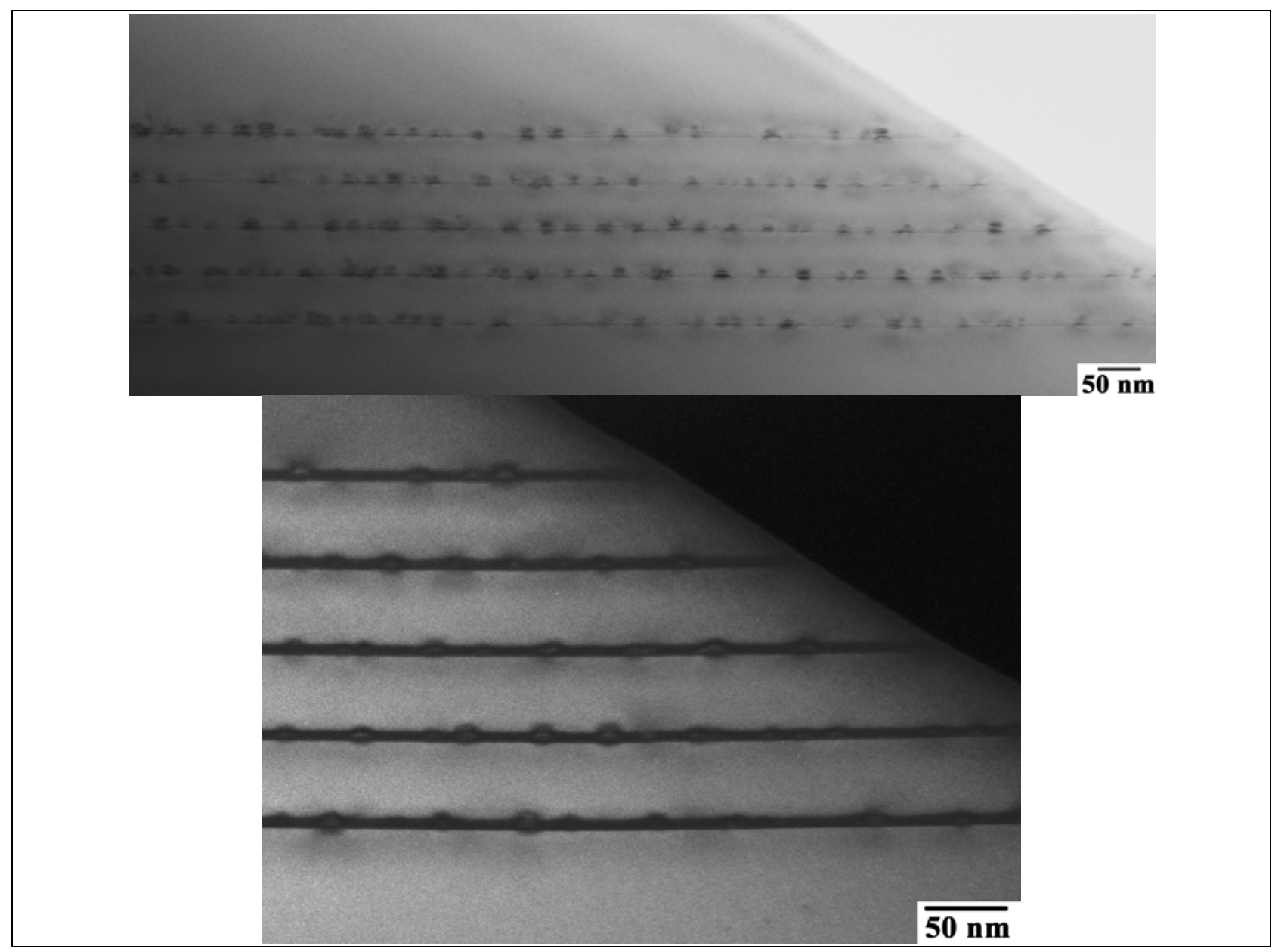

Figure 27. Cross-sectional TEM images of the 5-layer QDIP structure showing (a) $G=(022)$ bright-field image and (b) (200) dark-field image.

Distribution Statement A. Approved for public release; distribution unlimited. 


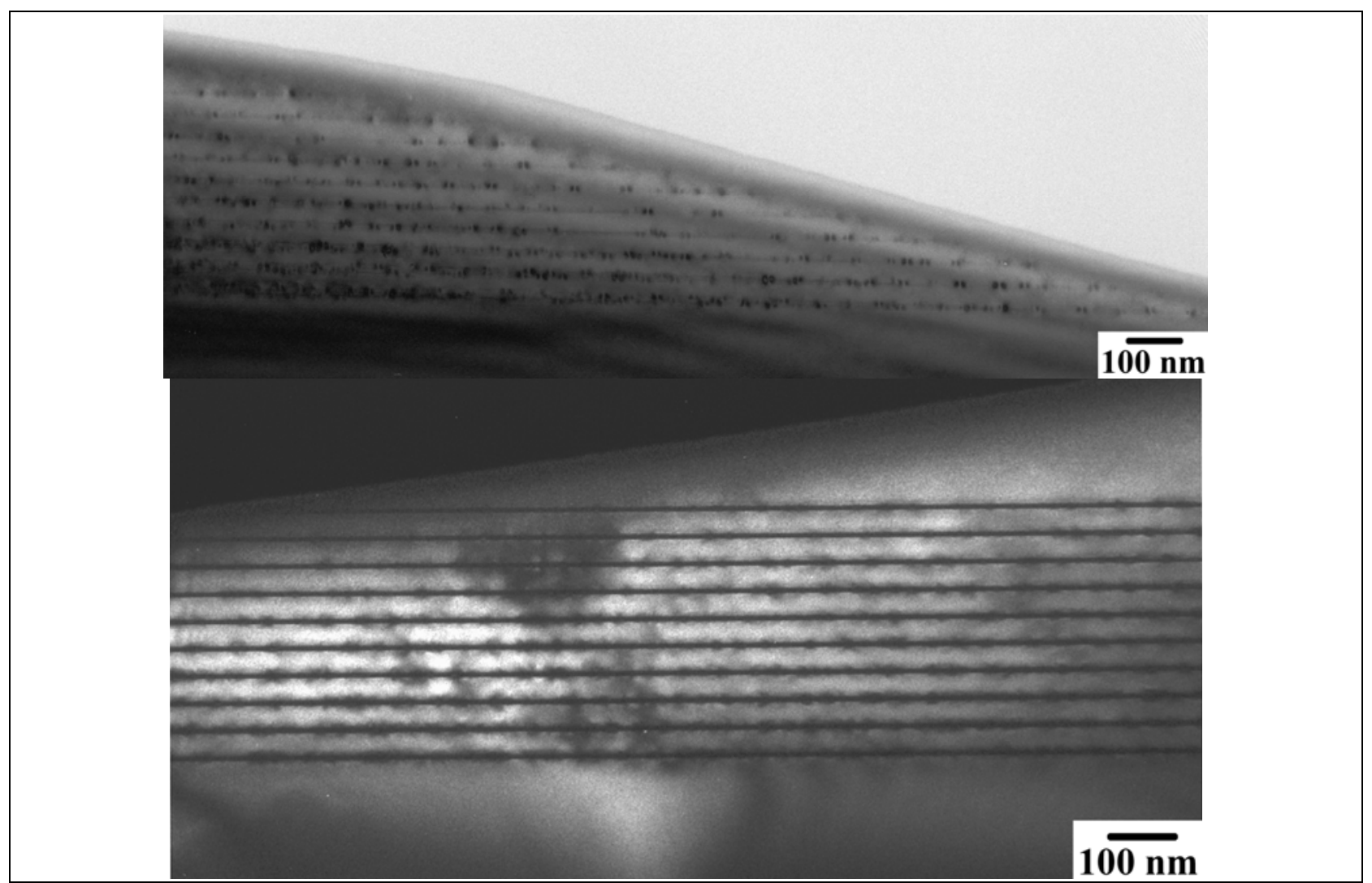

Figure 28. Cross-sectional TEM images of the 10-layer QDIP structure showing (a) $\mathbf{G}=(022)$ bright-field image and (b) (200) dark-field image.

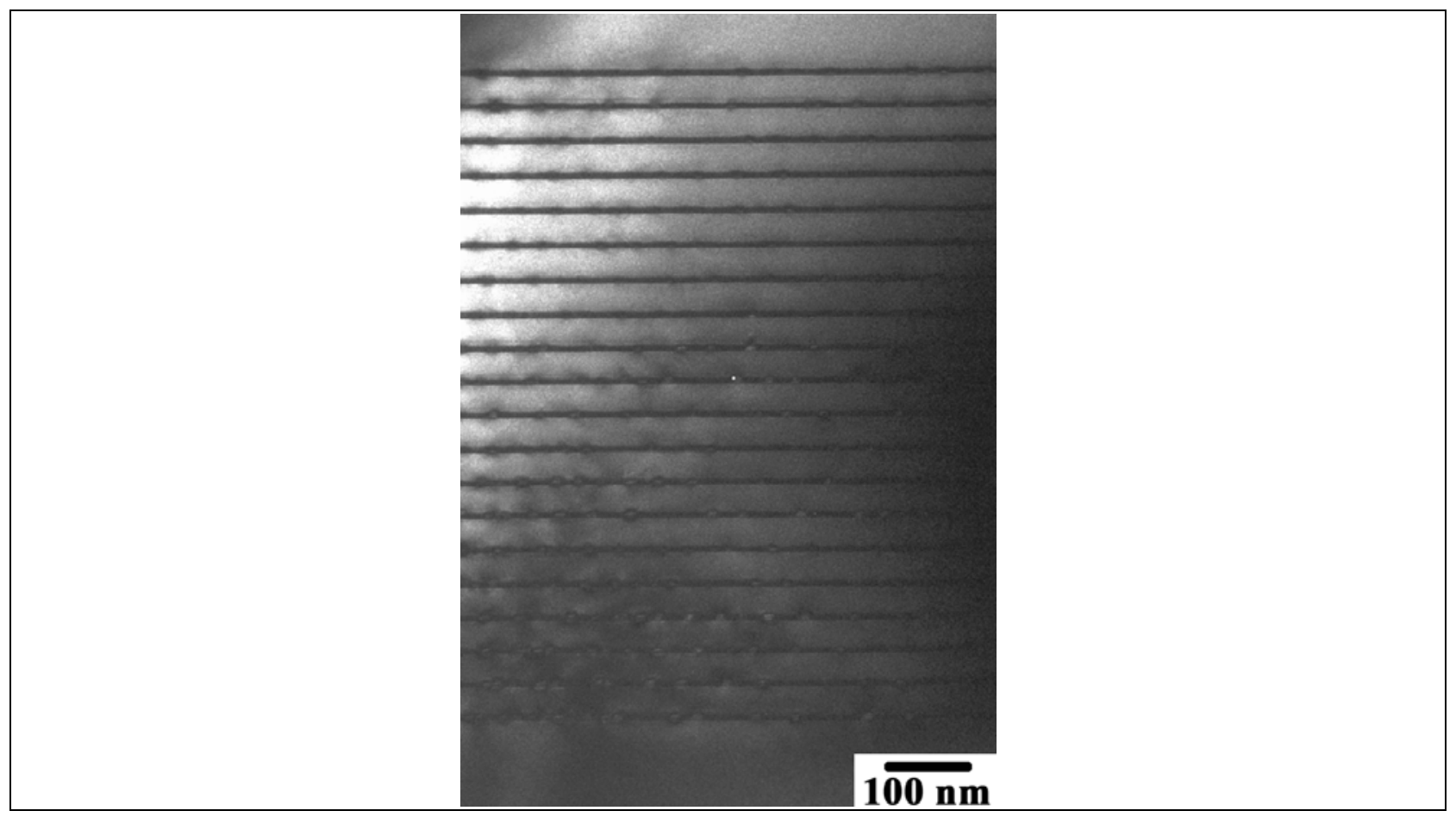

Figure 29. Cross-sectional TEM images of the 20-layer QDIP structure showing the (200) dark-field image.

Distribution Statement A. Approved for public release; distribution unlimited. 


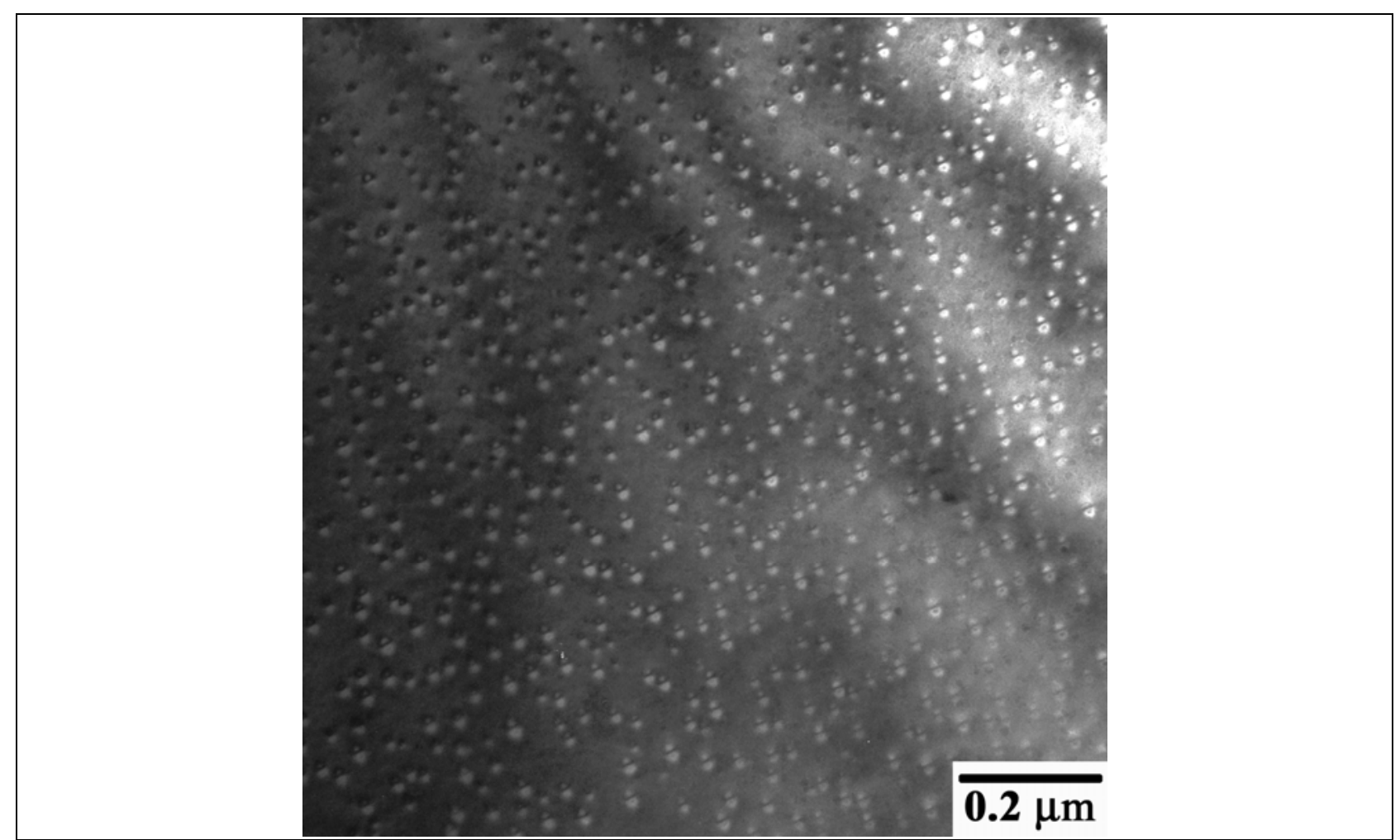

Figure 30. Plan-view TEM bright-field $(G=(220))$ image of the single-layer QDIP structure.

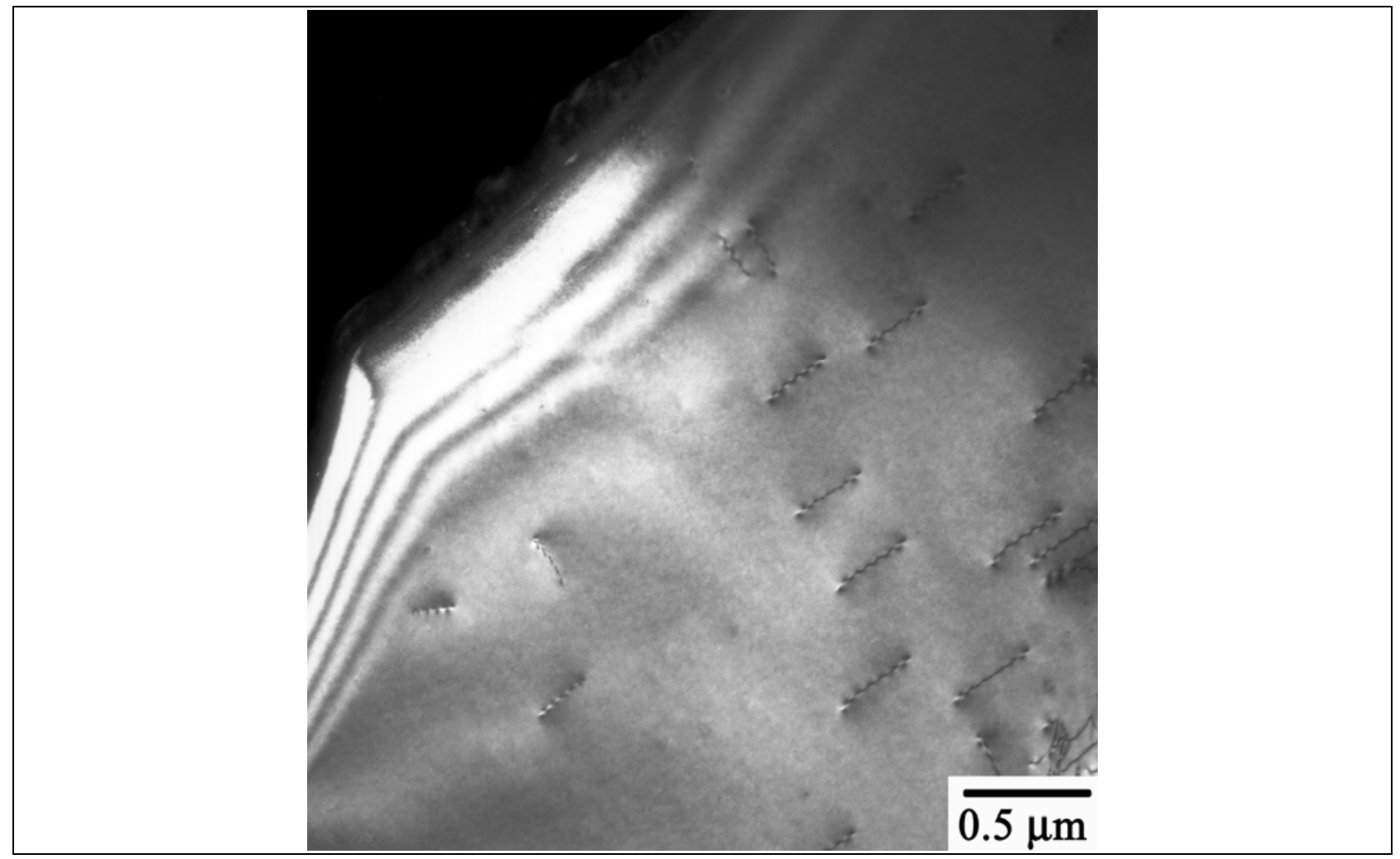

Figure 31. Plan-view TEM bright-field $(G=(220))$ image of the 10-layer QDIP structure with the top GaAs-contact layer.

Distribution Statement A. Approved for public release; distribution unlimited. 
Figure 32 is a (220) dark-field plan-view TEM image of the sample containing 20 QD layers. In this case the QDs are clearly visible because the top GaAs contact layer was etched away prior to TEM observations. A comparison with the image of the single layer QD layer (Figure 30) shows that the two structure are similar, although a higher density of QDs are observed in the 20-layer structure in the thicker regions (right hand side in Figure 32) due to the overlap in layers when image in plan-view. The absence of dislocations observed in this sample indicates that the source of high dislocation density observed in the 10-QD layer sample could be the top GaAs contact layer. To verify this possibility the plan-view TEM observations on the 10-QD layer sample was repeated after the top GaAs contact layer was etched off. The (220) dark-field image, shown in Figure 33, reveals that the dislocations observed in Figure 30 are removed indicating that they originated during growth of the GaAs contact layer. Also, the QDs in this image are similar to those observed in the other plan-view TEM images (Figures 30 and 32), thereby indicating that the defect density in the QD stack is low, as in the single layer and 20-layer QDIP samples.

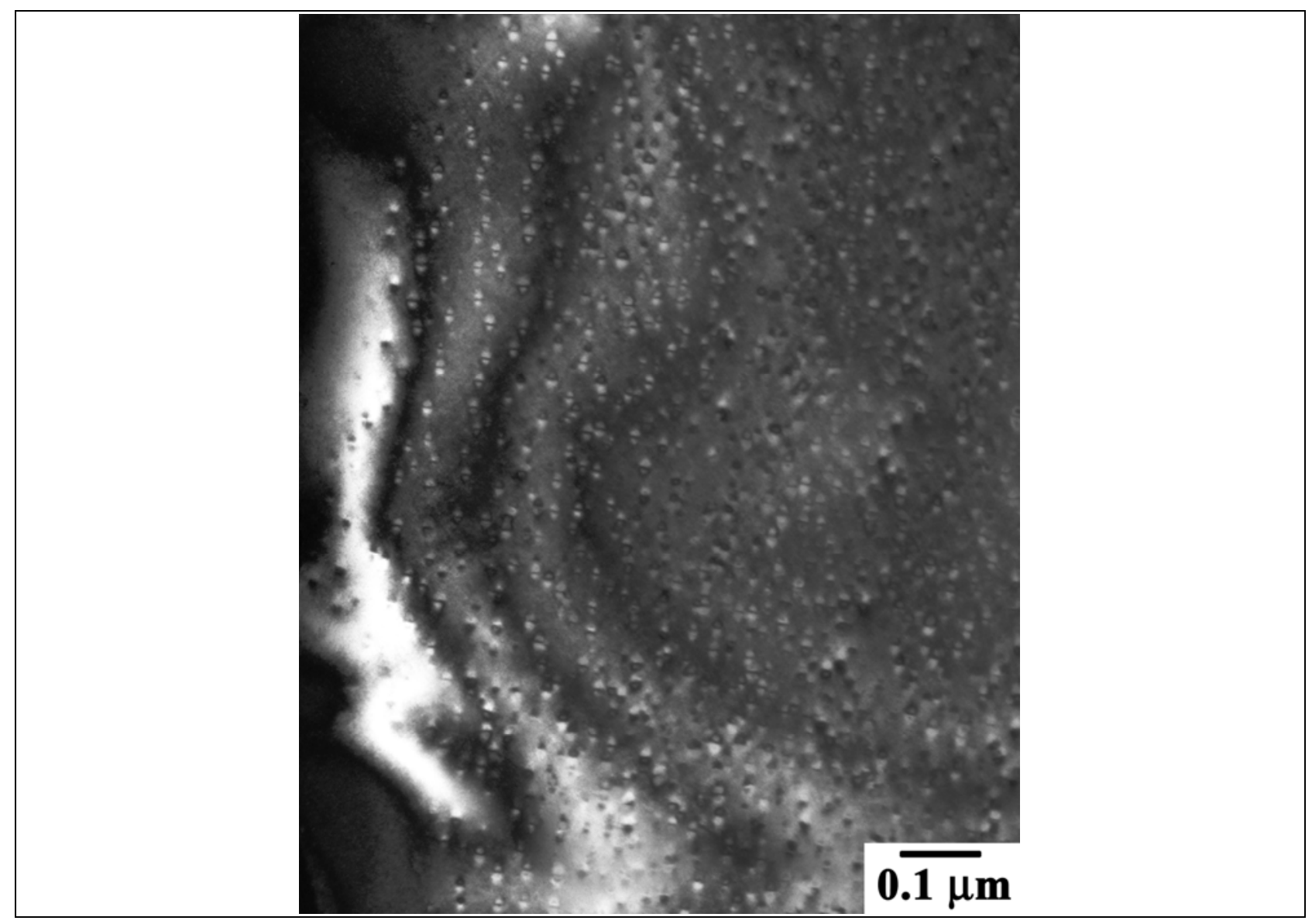

Figure 32. Plan-view TEM bright-field $(\mathrm{G}=(\mathbf{2 2 0}))$ image of the 20-layer QDIP structure with the top GaAs-contact layer removed.

Distribution Statement A. Approved for public release; distribution unlimited. 


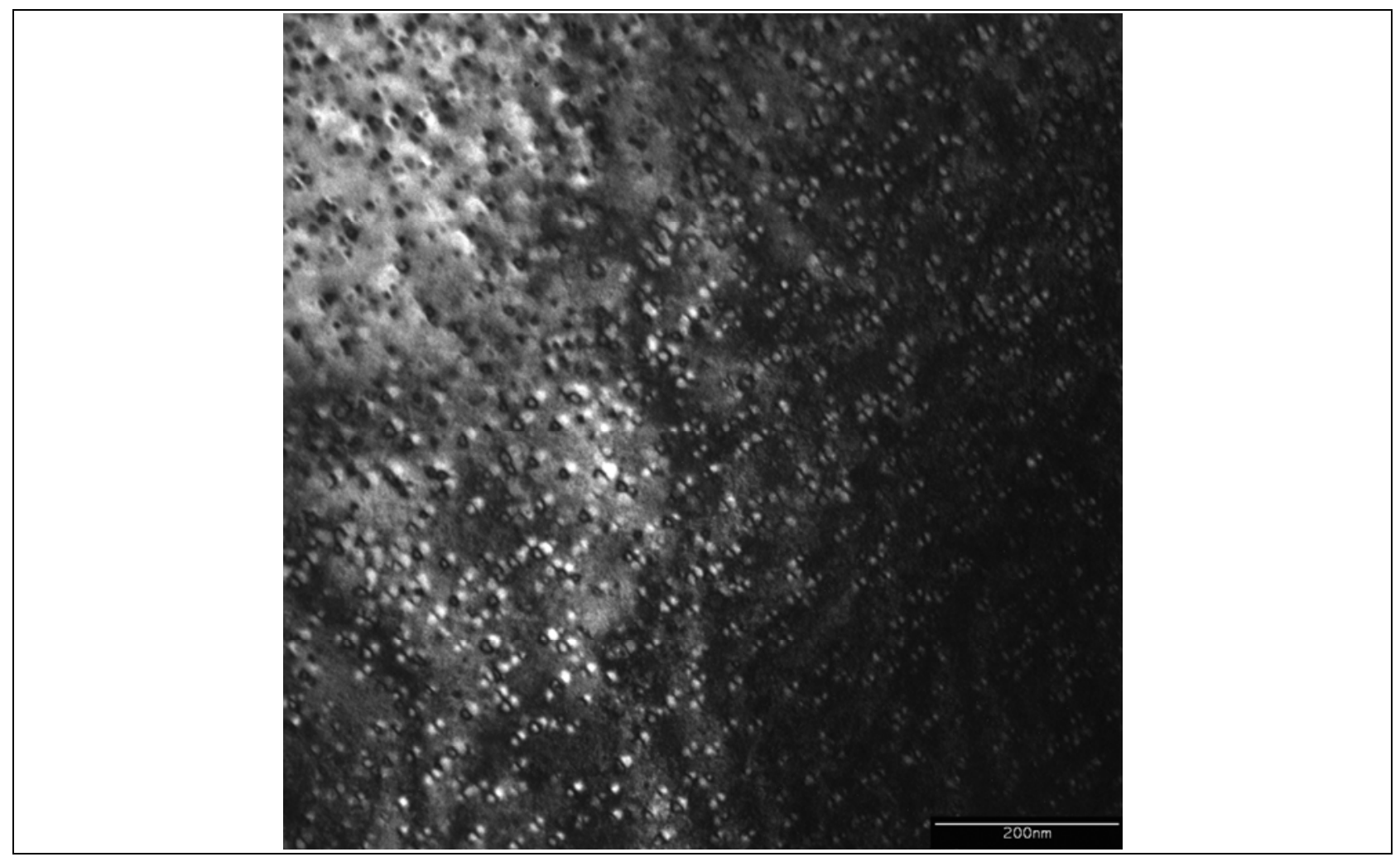

Figure 33. Plan-view TEM bright-field $(G=(220))$ image of the 10-layer QDIP structure with the top GaAs-contact layer removed.

In summary, detailed examination of InGaAs QDIP structures show that it is possible to achieve high quality multilayered stack with up to 20 layers. Optimization of growth parameters is however necessary to avoid dislocation generation during growth of the top GaAs contact layer. A modeling study employing the finite-element method is in progress to examine the influence of capping layer on strain propagation in structures with multiple QD stacks.

\subsection{Photoluminescence of PbS quantum dots on semi-insulating GaAs}

B. Ullrich ${ }^{1,2}$, X. Y. Xiao ${ }^{1}$, G. J. Brown ${ }^{1}$

${ }^{1}$ Air Force Research Laboratory, Materials \& Manufacturing Directorate, Wright Patterson AFB, OH 45433-7707

${ }^{2}$ Department of Physics and Astronomy, Centers for Materials and Photochemical Sciences, Bowling Green State University, Bowling Green, OH 43403-0209

We studied the emission properties of colloidal $\mathrm{PbS}$ quantum dots $(5.3 \mathrm{~nm})$ dispersed on semiinsulating GaAs in the temperature range of $5 \mathrm{~K}-300 \mathrm{~K}$ by employing Fourier transform infrared photoluminescence spectroscopy. The results reveal that the $\mathrm{PbS}$ quantum dots (QDs) alter and notably enhance the emission features of the GaAs substrate itself. The dependence of the QD emission peak position on temperature is modeled equivalently well with the well-known empirical Varshni equation and with a relation based on thermodynamics. The work reveals that emission properties of $\mathrm{PbS}$ quantum dots do not follow predictably general rules but are determined sensitively by the preparation method and substrate used.

Distribution Statement A. Approved for public release; distribution unlimited. 


\section{Introduction}

Potential applications in tunable laser sources, light emitting diodes, and display arrays, move the emission properties of nano-structured lead sulfide $(\mathrm{PbS})$ into the center of ongoing research activities. ${ }^{1}$ However, before the mastery of the emission of chemistry based $\mathrm{PbS}$ quantum dots might mature enough to serve in active device structures further basic questions have to be addressed. Stability of the emission is one issue, ${ }^{2}$ while another technologically important matter is the integration of PbS QDs with commonly used semiconductor layers and substrates such as Gallium Arsenide (GaAs) and related compounds, which are employed in mass-market optoelectronic devices. So far, most of the PL investigations of PbS QDs were performed by using glass hosts or glass carriers. ${ }^{1,3-6}$ In this paper, we address the matter of possible integrated hybrid $\mathrm{PbS} / \mathrm{GaAs}$ device formation by first investigating photoluminescence (PL) of PbS QDs on commercially available GaAs substrates. Particularly, we focused our activities on the PL temperature dependence and, additionally, we reveal that the presence of PbS QDs alters the emission properties of the GaAs substrate itself.

\section{Sample preparation}

The PbS QDs, with an average size of $5.3 \mathrm{~nm}$, were purchased from Evident Technologies. The QDs are dissolved in Toluene and capped by Oleic acid. The QDs were dispersed by a supercritical $\mathrm{CO}_{2}$ fluid process ${ }^{7}$ on $6 \mathrm{~mm} \times 6 \mathrm{~mm}$ pieces of a standard semi-insulating (SI) GaAs wafer. Before the QD deposition, the GaAs substrates were thoroughly rinsed with Isopropanol and Toluene, and afterwards, dried with nitrogen gas.

For the supercritical fluid $\mathrm{CO}_{2}$ deposition process, a high-pressure stainless steel chamber with a volume of about $16 \mathrm{~cm}^{3}$ was used. The chamber was carefully cleaned with acetone and high pressure $\mathrm{CO}_{2}$ liquid or gas. A glass container with about $250 \mu \mathrm{L}$ of the QD solution was set inside the chamber and two pieces of the GaAs substrates were inserted into the solution. One of the GaAs pieces was covered with a transmission electron microscopy (TEM) grid, which was used to estimate the surface coverage and size distribution of the QDs. The chamber was then purged at room temperature with $\mathrm{CO}_{2}$ gas at $70 \mathrm{~atm}$. The pressure was slowly increased to 100 atm. through a pressure modulator. After closing the inlet valve, the chamber was then slowly heated to $38^{\circ} \mathrm{C}$, while the inside pressure was increased to about $150 \mathrm{~atm}$. The chamber was kept at this condition for about 5 hours to allow the QDs to dissolve into supercritical $\mathrm{CO}_{2}+$ toluene liquid phase, and afterwards, the chamber was vented. The QDs deposited on both the GaAs substrate and the TEM grid surfaces were imaged by a scanning electron microscope (SEM). A typical result is shown in Figure 34. The arrangements of the QDs are rather random, but no multiple layers or three-dimensional aggregates are observed over the entire surfaces. It is evident that the QDs films deposited by this method from a supercritical fluidic environment are much smoother and more uniform than those deposited from solvent evaporation.

Distribution Statement A. Approved for public release; distribution unlimited. 


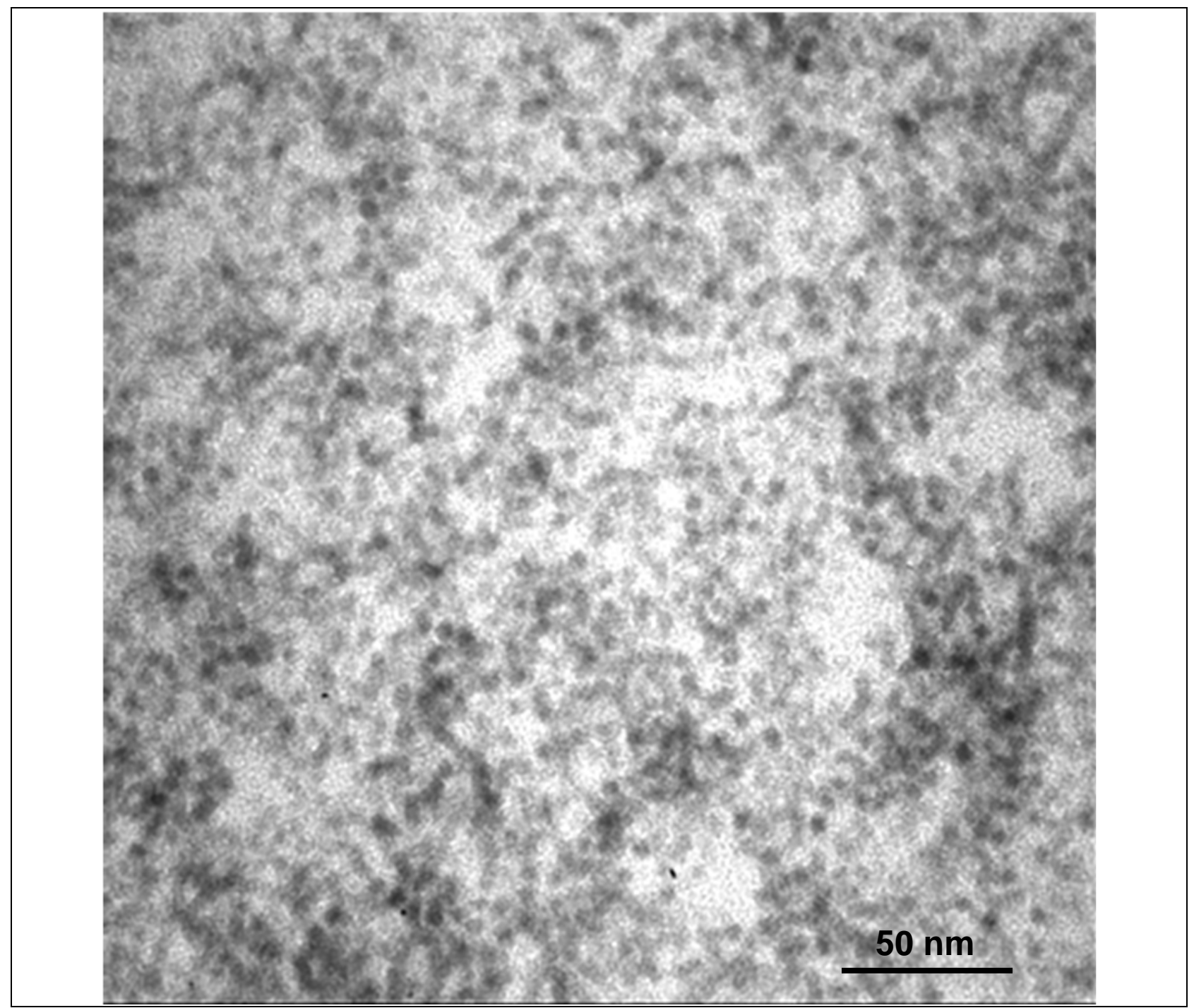

Figure 34: SEM image of the quantum dots. The bar on the right hand side corresponds to $50 \mathrm{~nm}$.

\section{Experiment}

The photoluminescence (PL) measurements were carried out by exciting the sample with the continuous wave (cw) $532 \mathrm{~nm}$ emission of a solid-state laser. The sample was mounted in an optical, liquid helium cooled cryostat equipped with a heater, allowing measurements from $5 \mathrm{~K}$ to $300 \mathrm{~K}$. A Fourier transform infrared (FTIR) Bomem spectrometer was used to measure the emission spectra. The expected emission of the QDs is around $0.9 \mathrm{eV}(1378 \mathrm{~nm})$ at room temperature. Around this spectral range the InGaAs detector would be the optimum choice for data collection. However, at low temperatures the QD PL peak emission shifts to lower energy and starts to move out of range of the InGaAs detector which has a cut-off wavelength of about $1550 \mathrm{~nm}(0.80 \mathrm{eV})$. Figure 35 illustrates the situation, showing that PL signals measured with the InGaAs detector exhibit a cut-off around $0.80 \mathrm{eV}$. Therefore, in order to provide full coverage of the PL peak of the QDs, an InSb detector was employed as well, resulting in two independent PL data sets from two different sample spots. Consequently, slight differences between measurements are not attributed to detector features but to the fact that the data are not collected

Distribution Statement A. Approved for public release; distribution unlimited. 
from exactly the same spot. Additionally, the signal-to-noise ratio is larger for the measurements with the InGaAs detector so the impinging laser intensity on the sample surface was increased from approximately $14 \mathrm{~W} / \mathrm{cm}^{2}$ and $80 \mathrm{~W} / \mathrm{cm}^{2}$ for the measurements carried out with the InGaAs and the InSb detector, respectively. This increase in laser intensity will shift the PL peak position $(<3 \mathrm{meV})$ and alter the temperature dependence slightly. ${ }^{8}$

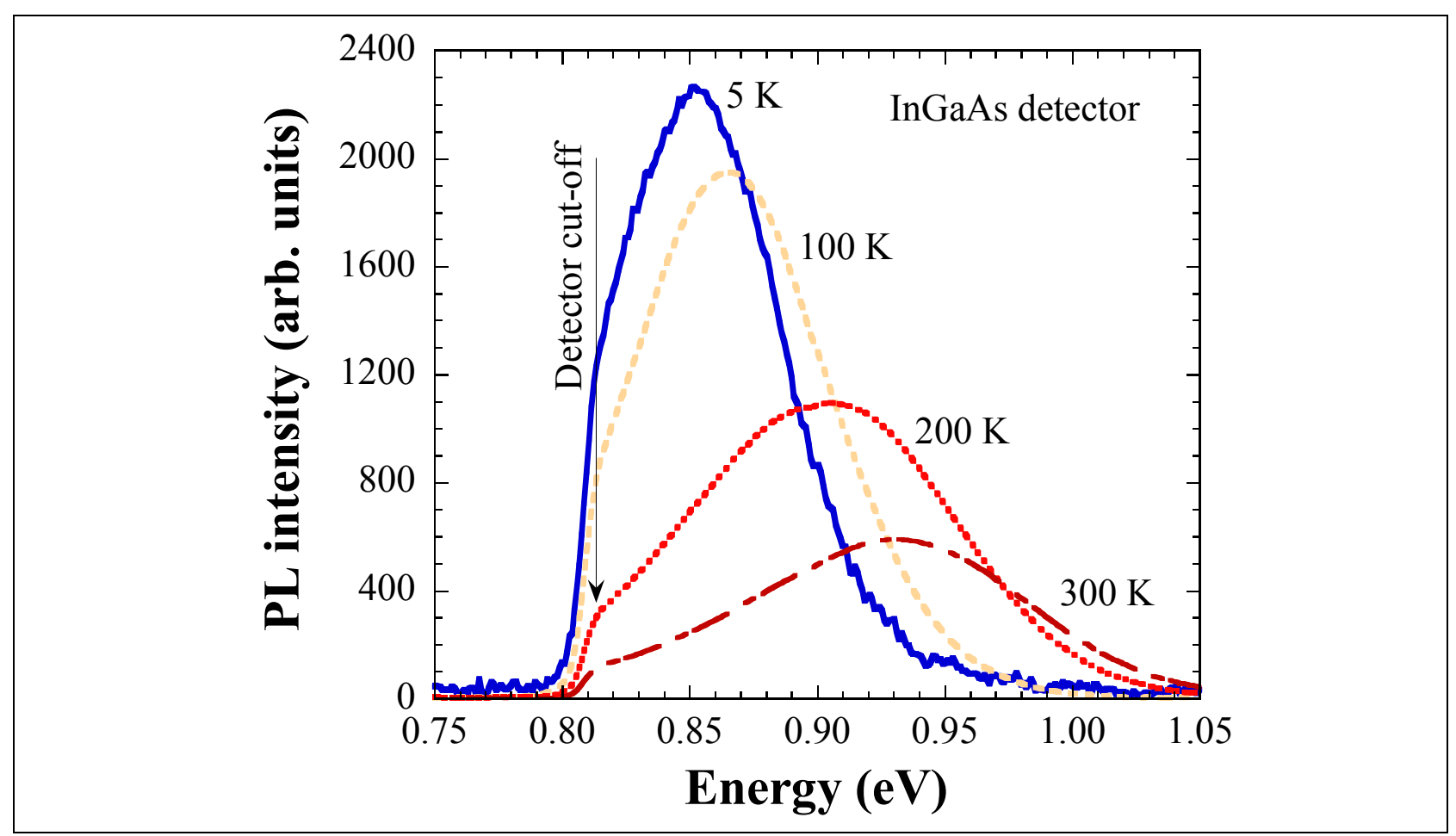

Figure 35: PL spectra of the PbS/GaAS sample at various selected temperatures measured with the InGaAs detector.

\section{Results}

Figure 36 shows the emission spectra at various temperatures measured with the InSb detector. The peak emission takes place in the range $0.86 \mathrm{eV}-0.95 \mathrm{eV}$, by varying the temperatures from $5 \mathrm{~K}$ to $300 \mathrm{~K}$. The shift of about $0.5 \mathrm{eV}$ from the bulk PbS band gap value at around $0.40 \mathrm{eV}$ (at $300 \mathrm{~K}$ ) indicates the highly effective quantum confinement of the PbS nanocrystals.

Additionally, the spectra show a broad emission peak centered around $0.65 \mathrm{eV}$. It originates from the well-known EL2 defect found in SI GaAs. ${ }^{9,10}$ PL spectra of EL2 related emission are routinely reported at 0.65 and $0.68 \mathrm{eV}$. What is not reported is the PL spectrum for SI GaAs at lower energies. So, the observed set of 3 peaks below $0.4 \mathrm{eV}$ is a new feature. Defect levels in SI GaAs below $0.4 \mathrm{eV}$ have been reported by other techniques. For example, Z.-Q. Fang et al. ${ }^{10}$ and Martin et al. ${ }^{11}$ reported levels in this range employing normalized thermally stimulated current analysis and Hall measurements, respectively.

The detailed features from 0.25 to $0.4 \mathrm{eV}$ in Figure 36 are GaAs related. This was checked by examining the shift of these peaks with temperature. These peaks shift to lower energy as the temperature is increased and follows the known temperature dependence of the GaAs band gap. Lead sulfide has the opposite temperature trend, i.e. the band gap increases with increasing temperature. The sharp peak at $0.37 \mathrm{meV}$ has a very narrow full-width at half maximum of 5 $\mathrm{meV}$ at $5 \mathrm{~K}$. Figure 37 shows the comparison of the PL spectra at $5 \mathrm{~K}$ from the bare SI GaAs

Distribution Statement A. Approved for public release; distribution unlimited. 
substrate and the sample with the deposited $\mathrm{PbS}$ quantum dots $(\mathrm{PbS} / \mathrm{GaAs})$ for energies below $1.1 \mathrm{eV}$. Notably, the PbS QDs enhance considerably the substrate PL intensities (a factor of about 5 and more), and enhances the intrinsic emission below $0.4 \mathrm{eV}$ revealing the additional peaks.

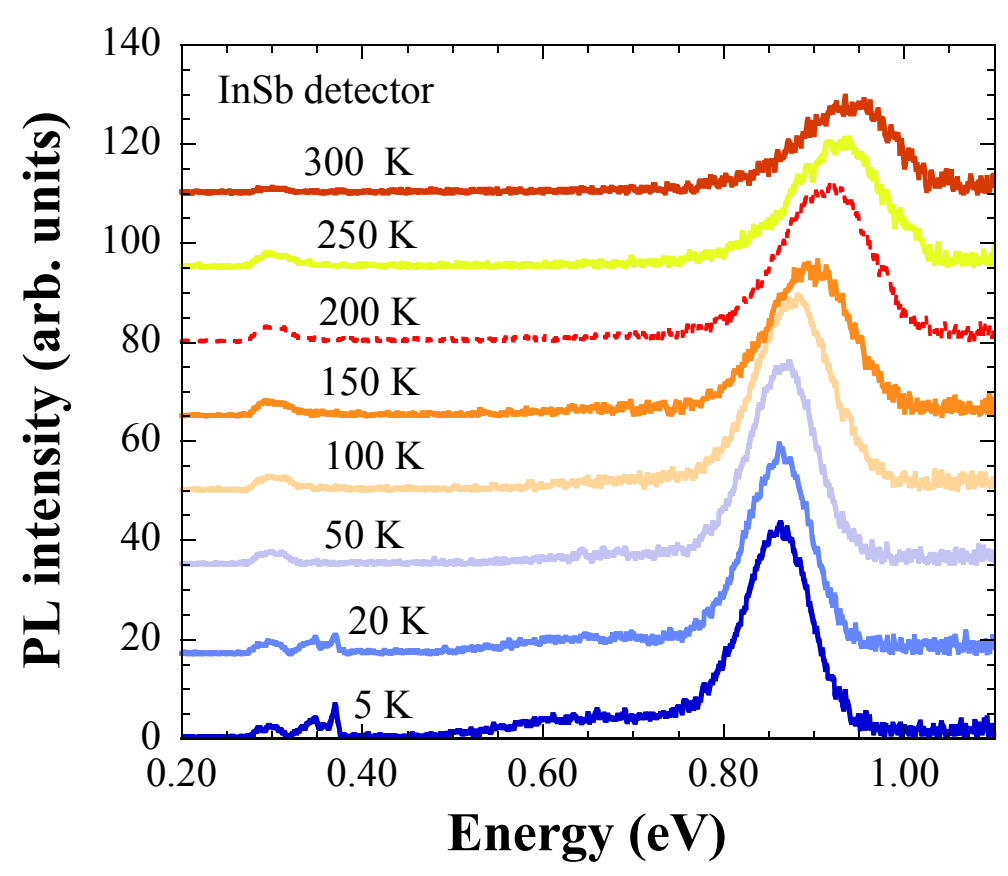

Figure 36: PL spectra of the PbS/SI GaAS sample at various temperatures measured with the InSb detector.

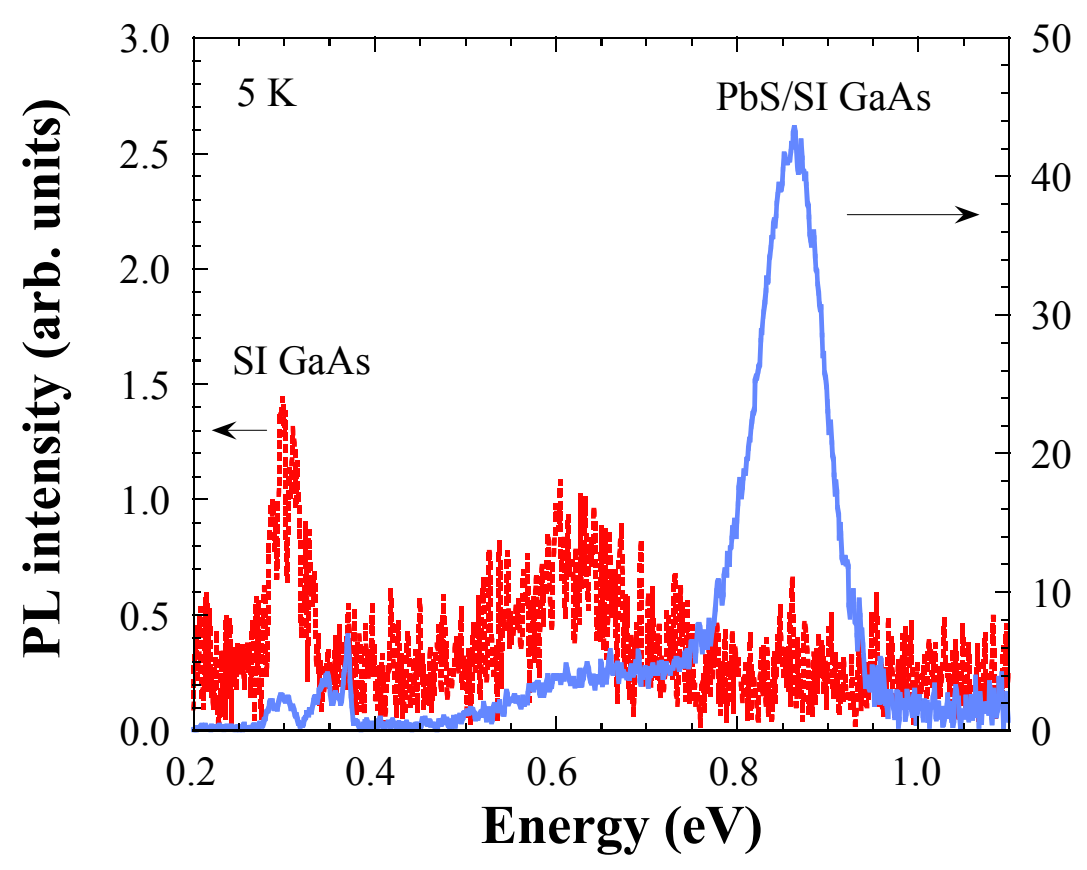

Figure 37: Comparison of the 5 K PL in the mid-infrared range of the SI GaAs substrate and the $\mathrm{PbS} / \mathrm{SI}$ GaAs sample. The spectra are shown in the same arbitrary units.

Distribution Statement A. Approved for public release; distribution unlimited. 
Similarly, when looking at the GaAs PL spectra near the band gap, utilizing the InGaAs detector, there are additional spectral changes when the PbS QDs are on the GaAs surface. Figure 38 shows the detailed comparison of the PL spectra at $5 \mathrm{~K}$ from the bare substrate and the $\mathrm{PbS} / \mathrm{GaAs}$ sample measured with the InGaAs detector. In this case, the band gap emission is not altered in position but the strong center peak due to donor-acceptor transition ${ }^{10}$ appears to be shifted by $2 \mathrm{meV}$ (from $1.4908 \mathrm{eV}$ to $1.4928 \mathrm{eV}$ ) towards higher energies. However, we interpret the PL shift not in terms of band gap energy alteration but due to a FWHM broadening of the PL peak caused by a carrier temperature increase in the SI GaAs substrate covered with PbS QDs with respect to the intrinsic bulk material. We further note that the transition at $1.5125 \mathrm{eV}$, which is due to a free exciton decay, ${ }^{10}$ does not reveal a notable FWHM change. However, the intensity of this transition is enhanced. These observations point to the fact that carriers are transferred from the QDs to the GaAs substrate, causing additional luminescent transitions in the near and mid-infrared regions and increasing the Coulomb interaction among carriers.

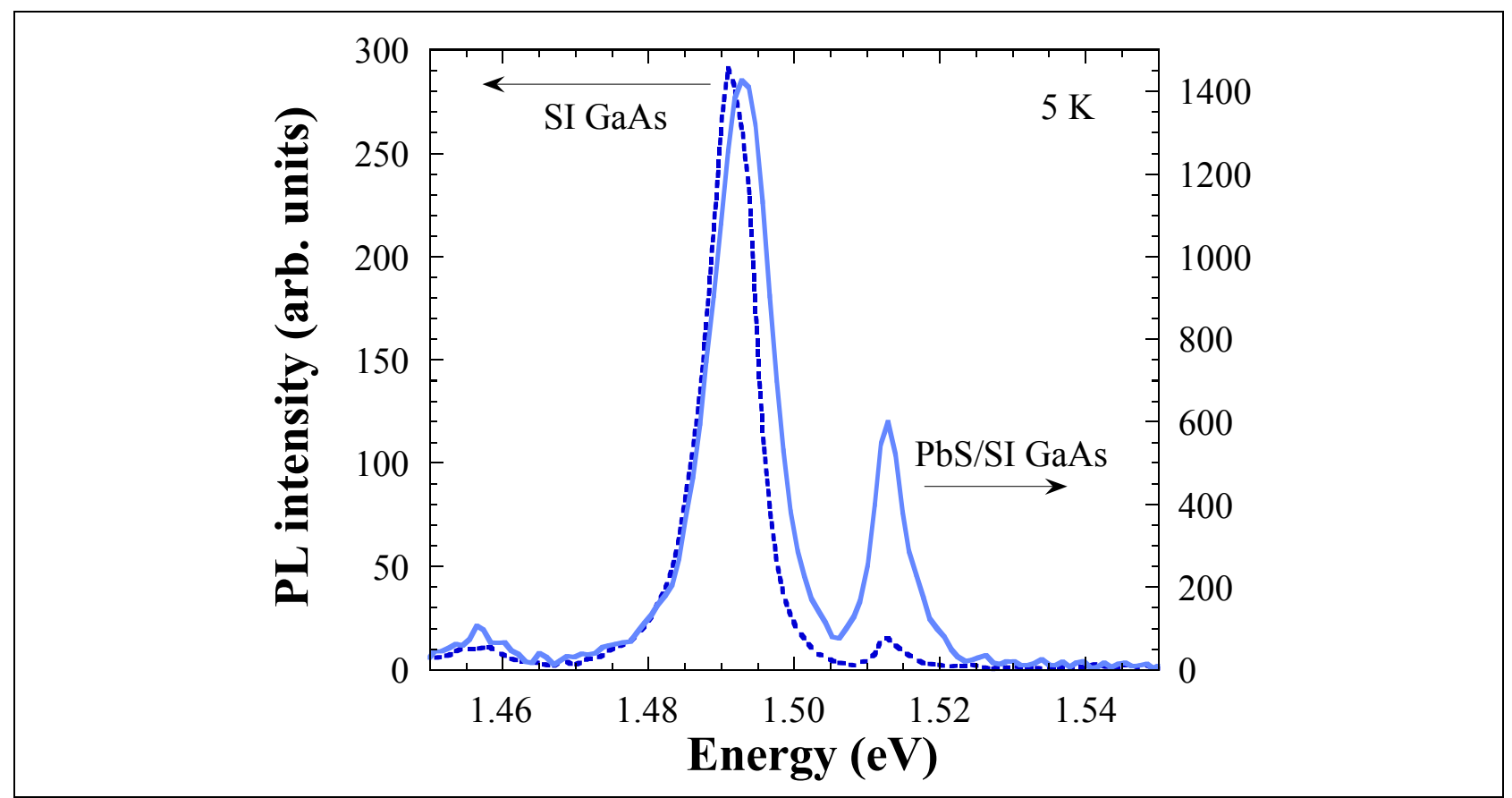

Figure 38: Comparison of the $5 \mathrm{~K}$ PL in the near infrared range of the SI GaAs substrate and the $\mathrm{PbS} / \mathrm{GaAs}$ sample. The spectra are shown in the same arbitrary units.

In addition to exploring the effects of the PbS QDs on the GaAs photoluminescence, we performed systematic temperature dependence studies of the QD PL peak. Figure 39, panels A and $\mathrm{B}$, show the energy position of the PbS QD emission maxima as a function of temperature as measured with both detectors. The overall temperature coefficient of the peak shift is about 0.3 $\mathrm{meV} / \mathrm{K}$, similar to the one of PbS QDs on glass above $150 \mathrm{~K} .{ }^{4}$ The shift of the QD PL peak in Figure 39A was fitted with the Varshni relation, ${ }^{12}$

Distribution Statement A. Approved for public release; distribution unlimited. 

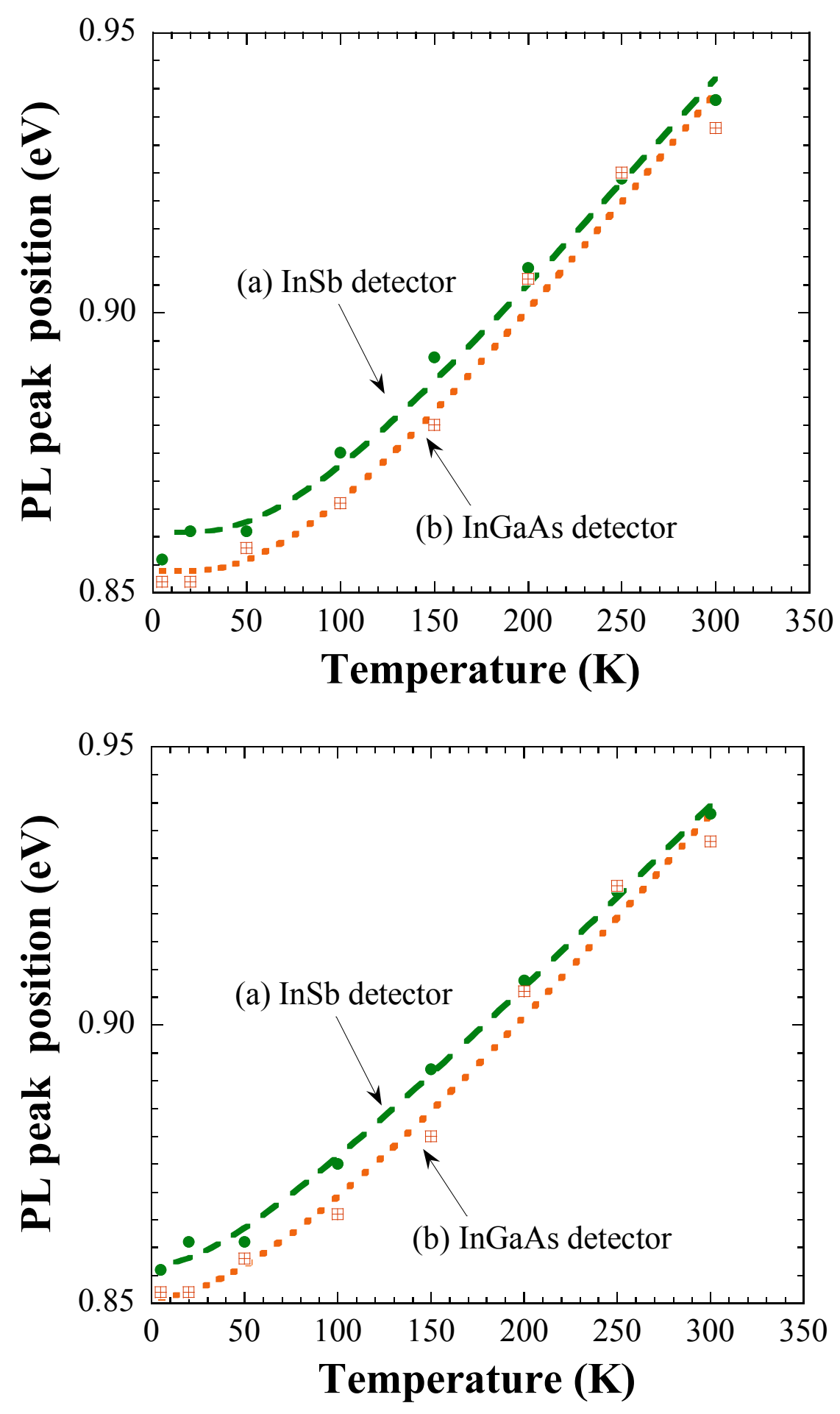

Figure 39: A) PL peak position vs. temperature measured (a) with the InSb detector and (b) with the InGaAs detector. The broken and dotted lines are fitted with Equation (19). B) PL peak position vs. temperature measured (a) with the InSb detector and (b) with the InGaAs detector. The broken and dotted lines are fitted with Equation (20).

Distribution Statement A. Approved for public release; distribution unlimited. 


$$
E_{\mathrm{g}}(T)=E_{\mathrm{g}}(0 \mathrm{~K})+\alpha T^{2} /(T+\beta),
$$

where, $E_{\mathrm{g}}$ is the band gap energy, $E_{\mathrm{g}}(0 \mathrm{~K})$ is the band gap energy at $0 \mathrm{~K}, T$ is the actual temperature, and $\alpha$ and $\beta$ are fitting parameters characteristic of a given material. Table 5 summarizes the fitting parameters used. For comparison, in Figure 39B, the PL peak shift was fitted employing the expression of O'Donnell and Chen, ${ }^{13}$

Table 5: Fitting parameters used with the Varshni relation

\begin{tabular}{|c|c|c|c|}
\hline Detector & $\boldsymbol{E}_{\mathbf{g}}(\mathbf{0} ~ \mathbf{~})$ & $\boldsymbol{\alpha}$ & $\boldsymbol{\beta}$ \\
\hline $\mathrm{InSb}$ & $0.8566 \mathrm{eV}$ & $0.000345 \mathrm{eV} / \mathrm{K}$ & 75.189 \\
\hline $\mathrm{InGaAs}$ & $0.8509 \mathrm{eV}$ & $0.000410 \mathrm{eV} / \mathrm{K}$ & 124.93 \\
\hline
\end{tabular}

$$
E_{\mathrm{g}}(T)=E_{\mathrm{g}}(0 \mathrm{~K})-S\left[E_{\mathrm{LO}}\right]\left(\operatorname{coth}\left\{E_{\mathrm{LO}} / 2 k T\right\}-1\right),
$$

where $S$ is a dimensionless coupling constant, $k$ is the Boltzmann constant, and $E_{\mathrm{LO}}$ is the LO phonon energy $(=26 \mathrm{meV}) .{ }^{4}$ The fitting parameters are shown in Table 6 . We note that the fits in Figure 39 indicate that the scatter of the PL peak energy vanishes at higher temperatures since thermal broadening and fluctuations dominate above possible inhomogeneous sample properties accumulated from different spots.

Table 6: Fitting parameters used with the O'Donnell-Chen relation

\begin{tabular}{|c|c|c|}
\hline Detector & $\boldsymbol{E}_{\mathbf{g}}(\mathbf{0} \mathbf{~ K})$ & $\mathbf{S}$ \\
\hline $\mathrm{InSb}$ & $0.8608 \mathrm{eV}$ & 1.3399 \\
\hline InGaAs & $0.8539 \mathrm{eV}$ & 1.4113 \\
\hline
\end{tabular}

It is worthwhile to stress that Equations (19) and (20) result in fits with a "goodness of fit value " exceeding 0.99 indicating an almost perfect agreement between theory and experiment. On the other hand, Kigel at al. ${ }^{14}$ reported for colloidal QDs that the Varshni relation does not predict the thermal PL peak shift for temperatures above $200 \mathrm{~K}$. Apparently, the temperature dependent emission properties of $\mathrm{PbS}$ QDs do not possess a general and easily predictable mechanism, depending more on preparation methods and carrier materials than on intrinsic properties such as the QD diameter.

Figure 40 reveals the full width at half maximum (FWHM) vs. temperature of the PL peaks measured with the InSb and InGaAs detector. The behavior varies from the reported one found with PbS QDs on glass. ${ }^{4}$ While the PL peak shift in Figure 39, from $5 \mathrm{~K}$ to $300 \mathrm{~K}$, is clearly larger in the current work ( $90 \mathrm{meV}$, rather than $55 \mathrm{meV}$ in Ref. 4), the temperature induced broadening of the FWHM remains smaller ( $35 \mathrm{meV}$ ) or comparable (54 meV), for the $\mathrm{InSb}$ and InGaAs detector, respectively, in comparison to published values in the range of 50-60 meV. The temperature dependence of the FWHM was fitted with the Bose distribution of function of the LO phonons, ${ }^{4}$

Distribution Statement A. Approved for public release; distribution unlimited. 


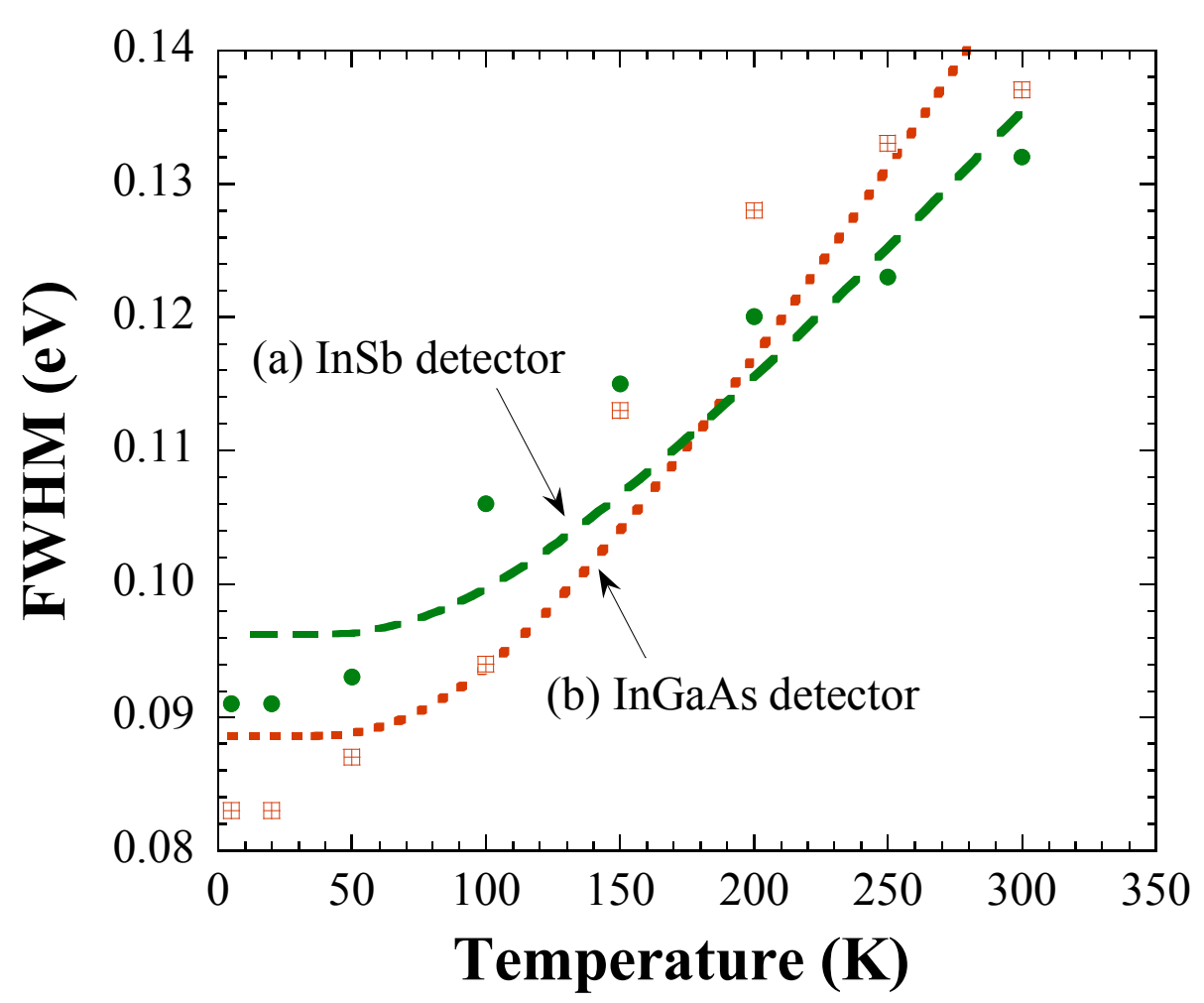

Figure 40: FWHM vs. temperature established with (a) the InSb detector and (b) the InGaAs detector. The broken and dotted lines are fitted with Equation (21).

$W=W(0 \mathrm{~K})+\gamma /\left[\exp \left(E_{\mathrm{LO}} / k T\right)-1\right]$

where, $W(0 \mathrm{~K})$ is the FWHM at $0 \mathrm{~K}$ and $\gamma$ is a fitting constant. The fitting parameters summarized in Table 7 are in reasonable agreement with the previously published values in Ref. 4.

Table 7: Fitting parameters used with the Bose phonon distribution function

\begin{tabular}{|c|c|c|}
\hline Detector & $\boldsymbol{W}_{\mathbf{0}}$ & $\boldsymbol{\gamma}$ \\
\hline $\mathrm{InSb}$ & $0.0962 \mathrm{eV}$ & $0.0680 \mathrm{eV}$ \\
\hline $\mathrm{InGaAs}$ & $0.0886 \mathrm{eV}$ & $0.0999 \mathrm{eV}$ \\
\hline
\end{tabular}

Figure 41 shows the PL intensity decrease with temperature increase of the sample. In contrast to the QDs on glass the intensity decrease reveals an overall linear decrease without the previously reported increase around $20-80 \mathrm{~K} .{ }^{4}$ The linear decay without a maximum at a specific temperature is attributed to a rather constant size of the luminescent QDs. ${ }^{15}$ The straight lines in Figure 41 represent a linear fit with close slope factors of -0.0203 and -0.0245 for the measurement carried out with (a) the InSb detector and (b) the InGaAs detector, respectively. We note that the room temperature PL peak intensity of about 25\% (Figure 35) and 35\% (Figure 36) with respect to the $5 \mathrm{~K}$ PL intensity clearly exceeds the $10 \%$ and $17 \%$ room temperature emission of $\mathrm{PbS}$ QDs on glass and colloidal QDs, respectively., 4

Distribution Statement A. Approved for public release; distribution unlimited. 


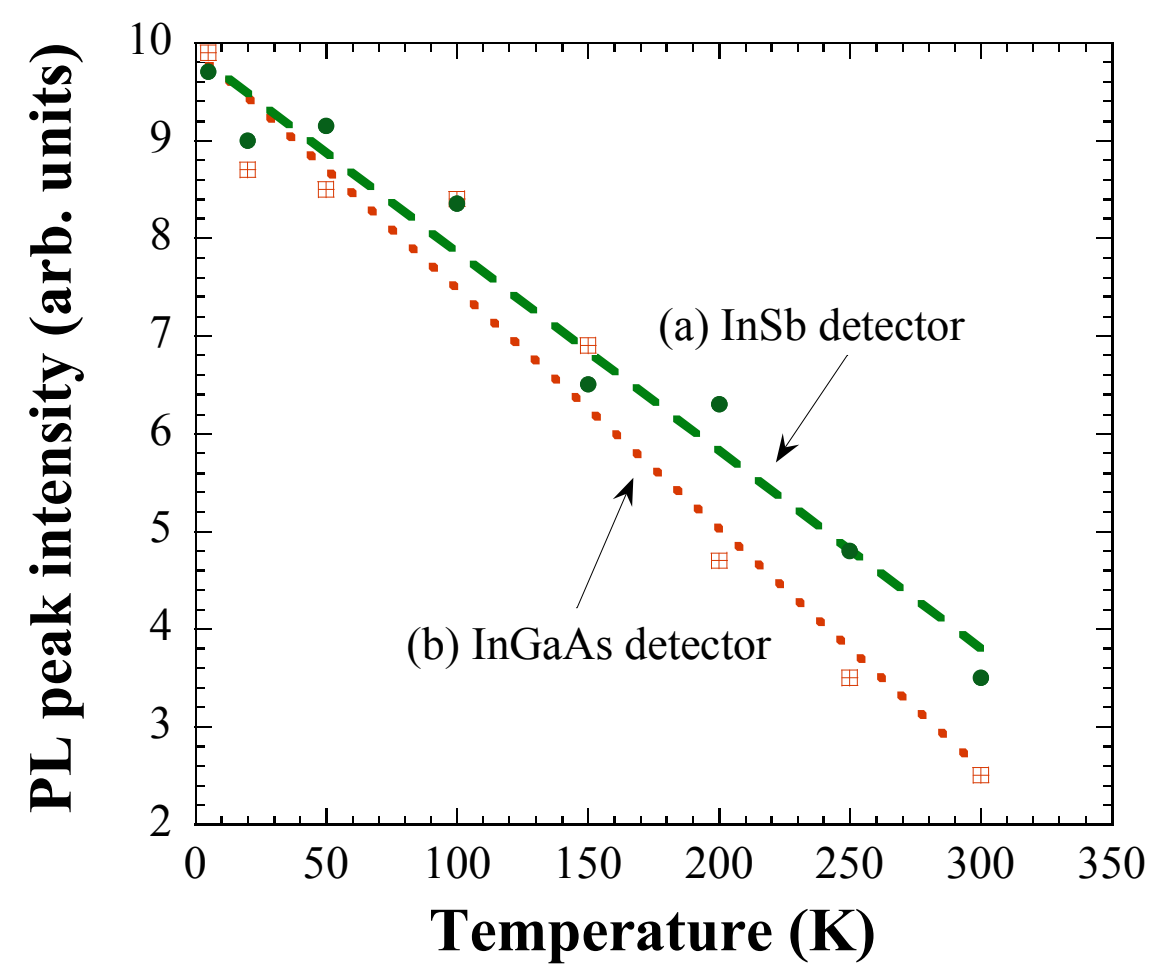

Figure 41: PL peak intensity vs. temperature measured with (a) the InSb detector and (b) InGaAs detector. The broken and dotted lines are linear fits of the data points.

\section{Summary}

In summary, the thermal dependence of the PL features of PbS QDs deposited on semi-insulating GaAs was investigated by FTIR spectroscopy. The study revealed that the deposition of PbS QDs alters the PL properties of the GaAs substrate because of charge transfer. Furthermore, we demonstrated that the temperature induced band gap shift can be fitted very well with the Varshni equation and a relation based on thermodynamic principles using the LO phonon energy of $26 \mathrm{meV}$. The latter was used as well in the Bose phonon distribution function in order to fit the FWHM of the PL peaks. The results stress that $\mathrm{PbS} / \mathrm{GaAs}$ hetero-pairings could impact optical and optoelectronic applications in two ways: first, the QD emission intensity is relatively strong at ambient conditions (up to $35 \%$ of the cryogenic maximum) and, second, for PL enhancement of GaAs based devices operating in the near infrared spectral range. In addition, the ability to easily transfer electrons (or holes) from the dispersed quantum dots to GaAs layers is an important building block for heterogeneous devices.

\section{References:}

1. C. Liu, Y. K. Kwon, and J. Heo, Appl. Phys. Lett. 94, 021103 (2009).

2. J. J. Peterson and T. D. Krauss, Nano Lett. 6, 510 (2006).

3. D. Kim, T. Kuwabara, and M. Nakayama, J. Lum. 119-120, 214 (2006).

4. L. Turyanska, A. Patane, M. Henini, B. Hennequin, and N. R. Thomas, Appl. Phys. Lett. 90,101913 (2007).

5. N. B. Pendyala and K. S. R. K. Rao, J. Lum. 128, 1826 (2008).

Distribution Statement A. Approved for public release; distribution unlimited. 
6. D. Kumar, G. Agarwal, B. Tripathi, D. Vyas, and V. Kulshresta, J. Alloys and Compounds 484, 463 (2009).

7. A. B. Smetana, J. S. Wang, J. J. Boeckl, G. J. Brown and C. M. Wai, J. Phys. Chem. C 112, 2294 (2008).

8. C. Liu, Y. K. Kwon and J. Heo, J. Mater. Sci.: 20, S282 (2009).

9. P. Kaminski, M. Pawlowski, R. Cwirko, M. Palczewska, and R. Kozlowski, Mat. Sci. Eng. B42, 213 (1996).

10. Z.-Q Fang, D. C. Reynolds, D. C. Look, N. G. Paraskevopoulos, and T. E. Anderson, J. Appl. Phys. 83, 260 (1998).

11. G. M. Martin, J. P. Farges, G. Jacob, J. P. Hallais, and G. Poiblaud, J. Appl. Phys. 51, 2840 (1980).

12. Y. P. Varshni, Physica 34, 149 (1967).

13. K. P. O’Donnell and X. Chen, Appl. Phys. Lett. 58, 2924 (1991).

14. A. Kigel, M. Brumer, G. I. Maikov, A. Sashchiuk, and E. Lifshitz, Small 5, 1675 (2009).

15. N. B. Pendyala and K. S. R. Koteswara Rao, J. Lumin. 128, 1826 (2008).

3.3 Surface, Crystallographic and Optical Properties of PbS QDs Formed with Supercritical Fluid $\mathrm{CO}_{2}$

Joanna S. Wang ${ }^{\dagger}$, Bruno Ullrich ${ }^{\dagger}$, Gail J. Brown ${ }^{\dagger}$, Chien M. Wai ${ }^{\dagger}$

${ }^{\dagger}$ Air Force Research Lab, Materials and Manufacturing Directorate, WPAFB, OH 45433-7707

${ }^{*}$ Department of Chemistry, University of Idaho, Moscow, Idaho 83844

\begin{abstract}
Lead sulfide (PbS) quantum dots (QDs) were synthesized in our lab with controllable and tunable sizes. Supercritical fluid $\mathrm{CO}_{2}\left(\mathrm{sc}-\mathrm{CO}_{2}\right)$ provides a useful tool to deposit $\mathrm{PbS}$ QDs on substrate surfaces with lateral uniformity. Either in the $\mathrm{PbS} /$ toluene solution or in the $\mathrm{sc}-\mathrm{CO}_{2}$ fabricated film, the absorbance maxima of the $\mathrm{PbS}$ QDs do not show an obvious dependence on the $\mathrm{PbS}$ concentrations. Fluorescence spectra of $\mathrm{PbS}$ QDs obtained from the films prepared by the sc- $\mathrm{CO}_{2}$ method indicate energy transfer between PbS QDs with different sizes. Samples formed with sc- $\mathrm{CO}_{2}$ method show red-shift and enhanced emission intensity with respect to samples formed with solution deposition method (SDM), specifically at cryogenic temperatures. Photoluminescence (PL) studies indicate that $\mathrm{PbS} /$ toluene solutions and films remain steady for several months without noticeable change in peak positions and intensities.
\end{abstract}

\title{
Introduction
}

The creation of nanoparticles of controllable size as building blocks for the assembly of supramolecular structures such as ordered arrays and nanoparticle films has been an area of considerable interests to material scientists in recent years [1-3]. Much of the interest has been driven by their prospective applications as electronic devices [4,5], optical materials [6,7], sensors [8,9], molecular catalysts $[10,11]$, and others [10,12]. Geometries of semiconductor structures are quickly approaching nano scales. It is generally accepted by the semiconductor community that the increasing complexity and decreasing dimensions of semiconductor devices require new materials deposition techniques. Current deposition technology such as chemical vapor deposition [13], physical vapor deposition [14], and electroplating [15] are becoming strained in their abilities to fill small structures of less than $100 \mathrm{~nm}$. A major obstacle to further

Distribution Statement A. Approved for public release; distribution unlimited. 
device shrinkage appears to be the ability to fill high-aspect-ratio, sub-100 nm structures. For this purpose, the use of supercritical fluid deposition technology appears promising to facilitate materials deposition for the sub-100 nm "nano" realm [12].

$\mathrm{PbS}$ nanoparticle colloidal solutions of quantum confined III-V semiconductor materials have been investigated due to the size-tunable optical properties, arising from the effect of quantum confinement [13-16]. In optoelectronics, the size tunability of quantum dots (QDs) allows the control over the spectral range of absorption, photoluminescence, stimulated emission and electroluminescence spectra [17-19]. Quantum confinement of charge carrier in nanocrystals whose size is comparable to their excitonic Bohr radius results in discrete energy level and narrow optical transitions.

Supercritical fluid $\mathrm{CO}_{2}$ is known to have near zero surface tension and provides an ideal medium for depositing nanoparticles in small structures to form ordered arrays, which cannot be achieved by traditional solvent deposition methods [1]. Its inertness, moderate critical parameters, low cost, and non-toxicity make sc- $\mathrm{CO}_{2}$ more appealing as an environmentally friendly medium for synthesis, deposition, and dissolution processes. For example, gold and platinum nanoparticles can be uniformly deposited in nanometer-sized trenches on silicon wafers forming ordered arrays in sc- $\mathrm{CO}_{2}[2,20] . \mathrm{Ag}_{2} \mathrm{~S}$ and $\mathrm{CdS}$ nanoparticles can be deposited in nanostructures of semiconductor substrates using $\mathrm{sc}-\mathrm{CO}_{2}$ as a medium [1]. In this study sc- $\mathrm{CO}_{2}$, as a medium was used for the deposition of QDs on substrates. Different sizes of PbS QDs were synthesized. The $\mathrm{PbS}$ films on glass substrate fabricated in a lab-made special apparatus were formed using sc- $\mathrm{CO}_{2}$ deposition and SDM. The spectroscopic studies such as UV-Vis-NIR, fluorescence and photoluminescence (PL) properties of the films formed by this method have been investigated.

\section{Experimental Section}

Chemicals. Hexanes, toluene, octadecene (ODE), bis(trimethylsilyl)sulfide (TMS), trioctylphosphine (TOP), oleic acid (OA), ethanol, and methanol, were purchased from Aldrich and used as received.

Synthesis of Semiconductor Nanoparticles. Oleic acid-capped PbS nanoparticles were prepared, in principle, in a similar manner to Hines et al. [21], but with some modifications. 0.5 $\mathrm{mL}\left(1.6 \times 10^{-3} \mathrm{~mol}\right)$ oleic acid, $0.09 \mathrm{~g} \mathrm{PbO}\left(0.4 \times 10^{-3} \mathrm{~mol}\right)$, and $16 \mu \mathrm{L}$ of bis(trimethylsilyl)sulfide (TMS) $\left(0.076 \times 10^{-3} \mathrm{~mol}\right)$, were used, as a molar ratio of $20: 5: 1$.

$\mathrm{PbO}(0.09 \mathrm{~g})$, trioctylphosphine $(2 \mathrm{~mL})$, octadecene $(5 \mathrm{~mL})$, and $\mathrm{OA}(0.5 \mathrm{~mL})$ were added in a reaction flask, in vigorously stirring, under a flow of continuous of argon (Ar) gas at $150{ }^{\circ} \mathrm{C}$ for 1 h. After $1 \mathrm{~h}$, TMS $(16 \mu \mathrm{L})$ dissolved in $1 \mathrm{~mL}$ of TOP were quickly injected at $150{ }^{\circ} \mathrm{C}$ into the reaction flask, while the magnetic stirrer was vigorously stirring and Ar gas was continuously passing through. The solution color was changed from colorless to dark brown immediately. The heating source was removed until the temperature reduced to $100-120^{\circ} \mathrm{C}$. The temperature under that condition was kept and the stirring was continuous under Ar gas for another hour.

For the large size $\mathrm{PbS}$ nanoparticle synthesis, $0.09 \mathrm{~g} \mathrm{PbS}$ plus $4 \mathrm{~mL}$ oleic acid were mixed without adding TOP or ODE. Ar gas passed through the reaction flask at $150{ }^{\circ} \mathrm{C}$ for $1 \mathrm{~h} .42 \mu \mathrm{L}$ TMS dissolved in $1 \mathrm{~mL}$ of TOP was quickly injected into the reaction flask. The injection

Distribution Statement A. Approved for public release; distribution unlimited. 
temperature was controlled at $150^{\circ} \mathrm{C}$. Particle growth temperature was controlled at $150{ }^{\circ} \mathrm{C}$ as well for $1 \mathrm{~h}$. After that the stirring bar was turned off, the reaction flask was lift from the heating source, and cool to room temperature.

Sample was washed by absolute 200 proof ethanol 3 to 4 times and methanol 1 time using a 8 $\mathrm{mL}$ glass vial until the dark brown lower organic phase disappeared, and black PbS QDs were completely precipitated. The nanoparticles were dried with a stream of $\mathrm{N}_{2}$ and then dissolved in toluene.

Instruments and Characterizations. Carbon coated copper grids purchased from Ted Pella were used to prepare samples for particle size determination employing TEM define. To define the TEM and HRTEM samples, a $4 \mu \mathrm{L}$ droplet of diluted $\mathrm{PbS}$ toluene solution was dripped onto a carbon coated copper grid, and then dried at room temperature. If a high population of $\mathrm{PbS}$ nanoparticles is required, after the previous drop was dried, more drops can be added consecutively. A JEM $2100 \mathrm{LaB}_{6}$ (Phillips CM 200 FEG TEM) operating at $200 \mathrm{KV}$ was used for both low and high resolution imaging. The average size of the $\mathrm{PbS}$ nanoparticles was obtained from the TEM images by counting at least 300 particles using a ImageJ software. UVVis-NIR spectra were obtained on a Cary 5000 Varian UV-Vis-NIR spectrophotometer using pre-deposited $\mathrm{PbS}$ nanoparticles on a piece of glass scanning from 400 to $1600 \mathrm{~nm}$. The scanning electron microscope (SEM) Sirion instrument is manufactured by FEI, Inc. with field emission gun (FEG) source. The following parameters were used: accelerating voltage of 10 $\mathrm{kV}$, spot size of 3 , and working distance $\sim 4.5 \mathrm{~mm}$. Powder X-ray diffraction (XRD) patterns were recorded on a Rigaku DMAX/B RU200 X-ray powder diffractometer with graphite monochromatized $\mathrm{Cu} \mathrm{K} \alpha(\lambda=0.15406 \mathrm{~nm})$. A scanning rate of $0.05^{\circ} \mathrm{s}^{-1}$ was applied to record the pattern in the $2 \Theta$ range of $20-90^{\circ}$. Fluorescence spectra were measured with a Horiba Jobin Yvon Nanolog 916B spectrometer equipped with an IGA 512 InGaAs near-IR detector. The PL of the samples have been recorded by Fourier transform infrared spectroscopy (FTIR) using a BOMEM spectrometer in conjunction with a liquid nitrogen cooled InSb detector. The optical excitation of the sample was performed with the continuous wave emission of a solid-state laser emitting at $532 \mathrm{~nm}$ keeping the excitation intensity of $85 \mathrm{~W} / \mathrm{cm}^{2}$. The signal was detected with a nitrogen cooled InGaAs detector attached to the BOMEM. In order to measure the PL features at cryogenic temperatures, the sample was mounted in a closed cycle optical He gas cryostat operating in the range $5 \mathrm{~K}-300 \mathrm{~K}$. An Axiotron optical microscope (brand: Zeiss) instrument was used to study the low magnification of surface morphology of the PbS QD films produced by sc- $\mathrm{CO}_{2}$ deposition and open air SDM. The film thickness was measured with DekTak $6 \mathrm{M}$ Stylus Profiler instrument.

\section{Results and Discussion}

Synthesis of PbS nanoparticles. In previous studies $\mathrm{PbS}$ nanoparticles were prepared using $\mathrm{PbO}$, and TMS in dilution solvent ODE [21]. A capping reagent, OA, was used to protect colloid $\mathrm{PbS}$ nanocrystals. The temperatures and the molar ratio of $\mathrm{OA} /$ dilution solvent can vary depending on the desired particle sizes. At higher injection and growth temperatures it will produce large particles [21]. The higher molar ratio of OA/ODE will produce large size $\mathrm{PbS}$ if the size of nanoparticles is drastically large. Fine tune of mean $\mathrm{PbS}$ nanoparticle size can be achieved by adjusting the injecting and growth temperatures [21,22]. For example, in our experiments, at a low temperature $\left(70^{\circ} \mathrm{C}\right)$ particle growth process, the size of nanoparticles was

Distribution Statement A. Approved for public release; distribution unlimited. 
smaller compared with the one at a higher growth temperature $\left(150{ }^{\circ} \mathrm{C}\right) . \quad \mathrm{H}_{2} \mathrm{~S}$ gas has been used to replace the TMS and synthetic temperature used was as low as $40{ }^{\circ} \mathrm{C}$ and consequently smaller $\mathrm{PbS}$ nanocrystals were fabricated [22].

Because TOP is more chemically reactive than ODE, and PbS has higher PL emission when TOP was involved in the synthetic procedure, comparing with ODE [23], TOP was then substituted for the ODE in the chemical reaction. The authors [23] also increased molar ratio of $\mathrm{Pb}: \mathrm{S}$ about $\sim 10: 1$, and increased solvent dilution factor, which gave a distinct difference between nucleation and the growth step.

Controlled nucleation and particle growth are important steps for synthetic procedure.

Nucleation must occur on a short time scale. Rapid injection into solvent at high temperature generates transient super saturation in monomers and induces a nucleation burst. A series of $\mathrm{PbS}$ nanoparticle products synthesized were from $2.0 \mathrm{~nm}$ to $14.4 \mathrm{~nm}$. The relationship of band gap energy of $\mathrm{PbS}$ nanoparticles versus particle sizes based on the hyperbolic band model fits very well with the experimental data, as previously reported [24]. Band gap energy values approach constant after particle sizes are beyond $6 \mathrm{~nm}$, suggesting quantum confinement effects of QDs will disappear after the particle sizes increase to $6 \mathrm{~nm}$ or more [24,25]. Due to this reason, we focus on the formation of $\mathrm{PbS}$ of less than $5 \mathrm{~nm}$ diameter.

The X-ray diffraction pattern of oleic acid capped $\mathrm{PbS}$ nanoparticles is given in Figure 42. All diffraction peaks can be indexed to a face-centered-cubic (fcc) PbS crystal structure. The sharp peaks indicate the product is well crystalline. All $\mathrm{PbS}$ nanoparticle samples show peaks at $2 \Theta=$ $25.96,30.06,43.06,50.98,53.4,62.54,68.88,70.96$, and $78.92^{\circ}$ which correspond to the (111), (200), (220), (311), (222), (400), (311), (420), and (422) planes of fcc structure of bulk PbS, respectively. The XRD pattern of the synthesized $\mathrm{PbS}$ nanoparticles agrees with the standard reference data [Joint Committee on Powder Diffusion Standards (JCPDS)] for PbS galena structure. In our XRD measurements shown in Figure 42, the PbS nanoparticles with the size in $14.4 \mathrm{~nm}$, have a cubic lattice structure and show evidence of a cubic appearance as well (Figure 43a). As particles become smaller they have a tendency to lose their cubic shape and become more and more spherical. They also have a tendency towards self-assembly.

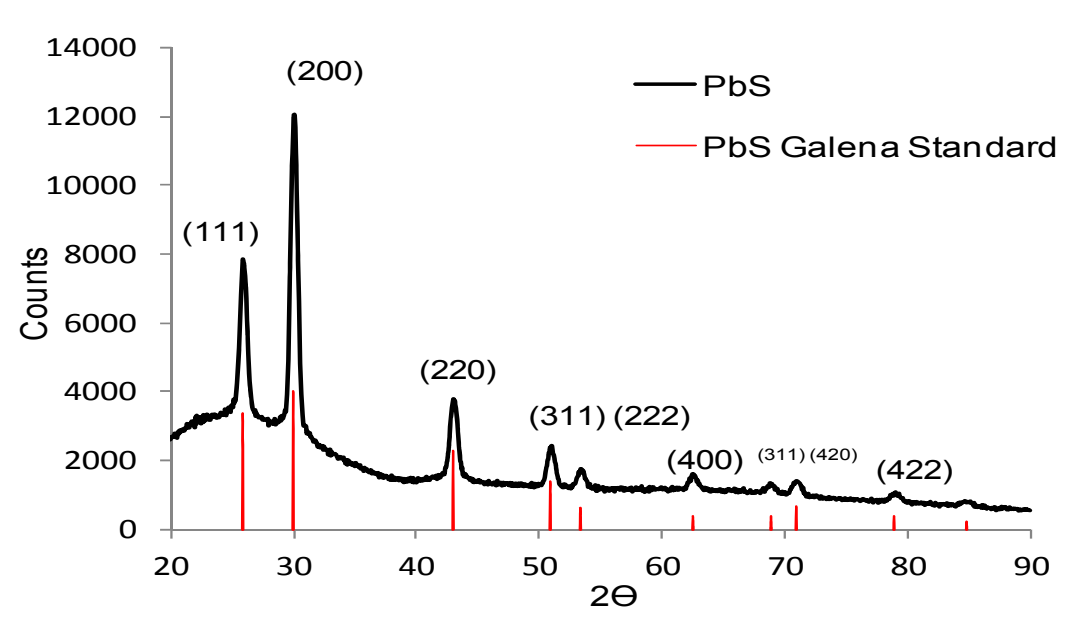

Figure 42. XRD pattern of PbS nanoparticles

Distribution Statement A. Approved for public release; distribution unlimited. 
Figure 43 shows TEM images of PbS with different sizes. PbS QDs synthesized in $14.4 \mathrm{~nm}$ are cubic lattice structures with tendency of self-assembly showing very ordered array (Figure 43a). Both $14.4 \mathrm{~nm}$ and $8.6 \mathrm{~nm}$ have no absorbance features in near infrared region. The $\mathrm{PbS}$ nanoparticles in $4.8 \mathrm{~nm}$ (Figure 43c) and $2.8 \mathrm{~nm}$ (Figure 43d) show strong absorbance in UVVis-NIR region, and strong fluorescence and PL intensities with quantum confinement effects.

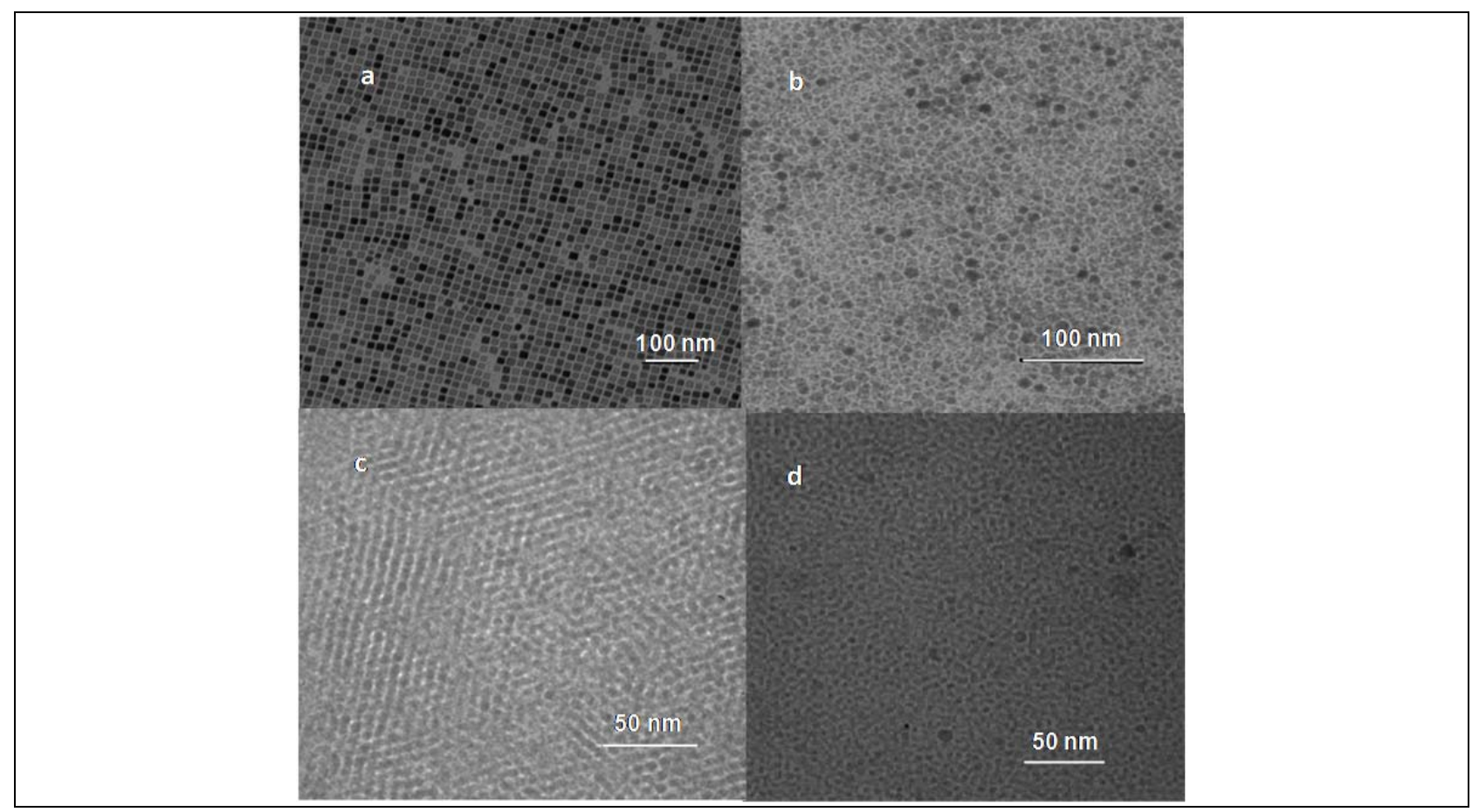

Figure 43. TEM images of PbS QDs with different sizes. (a) size $=14.4 \pm 1.6 \mathrm{~nm}$; (b) $\operatorname{size}=$ $8.6 \pm 1.1 \mathrm{~nm} ;(\mathrm{c})$ size $=4.8 \pm 0.54 \mathrm{~nm} ;(d) \operatorname{size}=2.8 \pm 0.31 \mathrm{~nm}$

\section{Deposition of $\mathrm{PbS}$ nanoparticles Using Supercritical Fluid $\mathrm{CO}_{2}$ as a Medium}

A typical supercritical fluid reaction system consists of a $\mathrm{CO}_{2}$ source (liquid $\mathrm{CO}_{2}$ tank), a syringe pump (ISCO model 260D) with a pump controller (ISCO Series D), high-pressure stainless steel cells, and a collection vessel. A high-pressure view-cell and a fiber-optic cell can also be connected to the system for visual observation and for in situ spectroscopic measurements. For deposition of nanoparticles on copper grids for TEM images, carbon coated copper grids are immersed in a toluene solution containing nanoparticles in a small glass container placed in a sc$\mathrm{CO}_{2}$ reaction cell preheated to a desired temperature as shown in Figure 44.

Distribution Statement A. Approved for public release; distribution unlimited. 


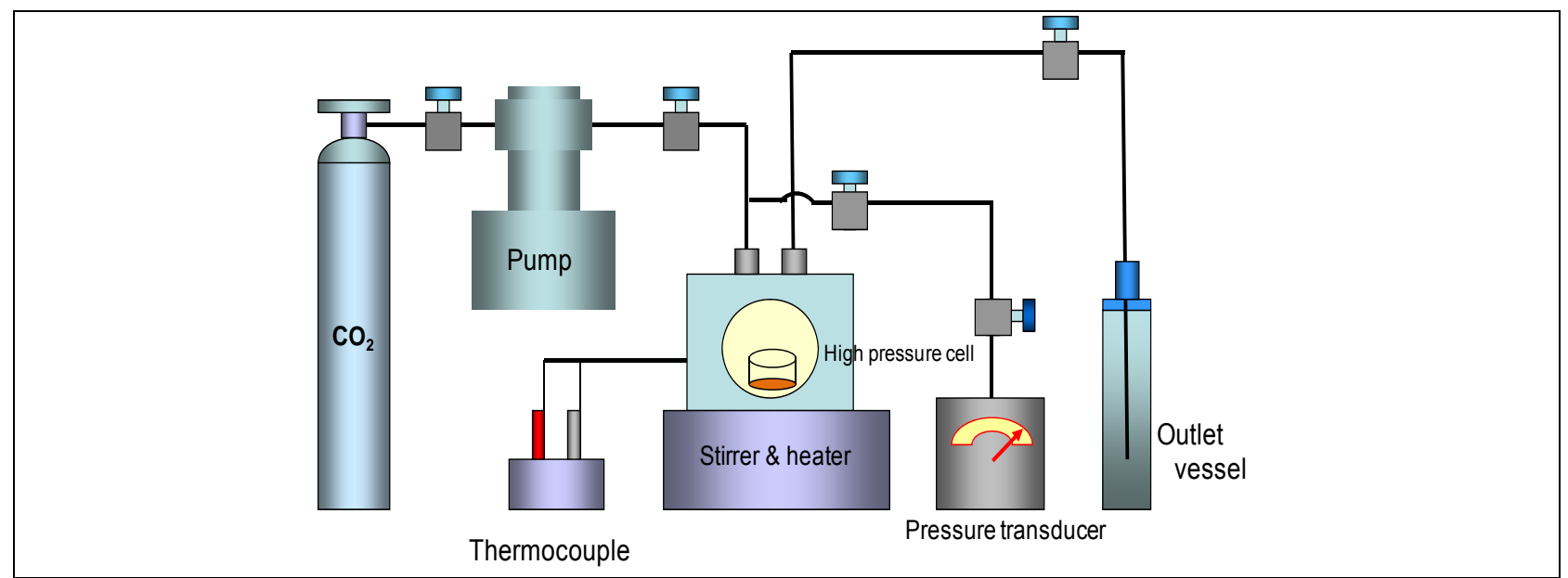

Figure 44. Supercritical fluid $\mathrm{CO}_{2}$ system for deposition of nanoparticles and formation of films

The sc- $\mathrm{CO}_{2}$ deposition process was carried out using a $35.3 \mathrm{~mL}$ high-pressure stainless steel chamber. Carbon coated copper grids or pieces of $\mathrm{Si}$ wafer were immersed in a $\mathrm{PbS}$ toluene solution placed in a small vial. The chamber was slowly charged with liquid $\mathrm{CO}_{2}(60 \mathrm{~atm})$ at room temperature over a period of $10 \mathrm{~min}$ and then the pressure was raised to $80 \mathrm{~atm}$. The system was then slowly heated to $40{ }^{\circ} \mathrm{C}$ to convert the liquid $\mathrm{CO}_{2}$ to the supercritical fluid phase. At this time the pressure inside the chamber was about $150 \mathrm{~atm}$. The ISCO pump then slowly raised the pressure up to $170 \mathrm{~atm}$ in the chamber. The high pressure apparatus was left at this condition $\left(40{ }^{\circ} \mathrm{C}\right.$ and $\left.170 \mathrm{~atm}\right)$ for $30 \mathrm{~min}$ to reach an equilibrium state. The reason to increase pressure from $150 \mathrm{~atm}$ to $170 \mathrm{~atm}$ is to ensure the system pressure is consistent and reproducible. The PbS nanoparticles would precipitate evenly and self-assemble to form a uniform 2-D array on the TEM copper grid or $\mathrm{Si}$ substrate in the sc- $\mathrm{CO}_{2}$ phase. The sc- $\mathrm{CO}_{2}$ deposition method was previously reported for making gold and metal sulfide nanoparticle arrays on Si substrate surfaces $[1,2,20]$ The particles are deposited by a gas-antisolvent (GAS) mechanism described previously in the literature $[2,26]$ where an increasing amount of $\mathrm{CO}_{2}$ alters the polarity of the toluene solvent and becomes unfavorable for particle stabilization in the colloid, that results in the particles precipitating from solution.

The oleic acid protected PbS QDs can also form arrays on the TEM copper grids immersed in the toluene solution under atmospheric pressure. However, the benchtop solvent evaporation process, due to high surface tension at the liquid/vapor interface, can lead to imperfect nanoparticle ordering forming isolated islands, percolating domains, locally high particle populations, and uneven surface coverage as described in the literature [26-29] (Figure 45a). On the other hand, if the deposition is carried out in $\mathrm{sc}-\mathrm{CO}_{2}$, the $\mathrm{PbS}$ QDs could form very organized and ordered arrays as shown in Figure 45b.

Distribution Statement A. Approved for public release; distribution unlimited. 


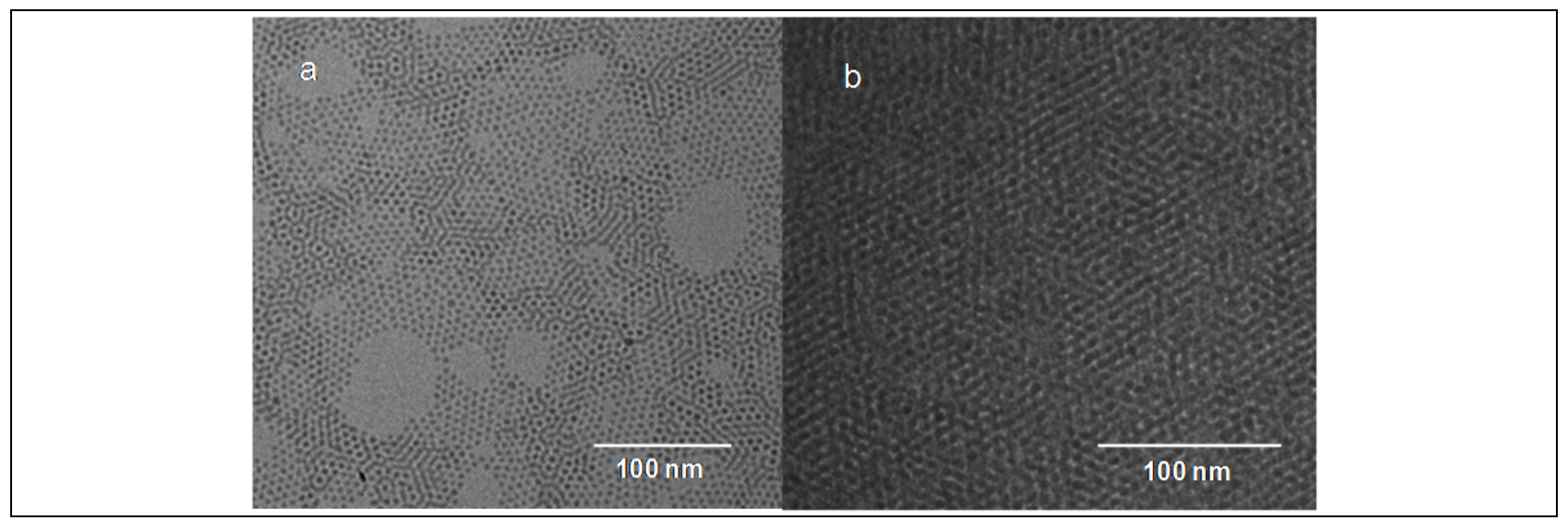

Figure 45. TEM images of the nanoparticle arrays formed by bench top solvent evaporation (a) and by supercritical fluid deposition (b) of the $4.7 \mathrm{~nm} \mathrm{PbS}$ nanoparticles.

For a systematic investigation of optical properties of $\mathrm{PbS}$ nanoparticle films, the composition and the amount of $\mathrm{PbS}$ nanoparticles in the sample films require to be quantitatively controlled. For this purpose, a sample forming apparatus was designed as described in Figure 46b for making uniform films with controllable composition and quantity of $\mathrm{PbS}$ nanoparticles. This apparatus is used for making nanoparticle films on flat substrate surfaces with solvent deposition (Figure 46c) and with sc- $\mathrm{CO}_{2}$ deposition (Figure 46d) and consequently making quantitative measurements in spectroscopic studies.

The apparatus consists of two pieces of aluminum plates each with 2 circular opening in the center ( $1 \mathrm{~cm}$ diameter) (Figure 46b). In order to increase the output of samples for comparing experiments, two metal cups on the apparatus were fabricated. A piece of flat substrate is inserted between the two aluminum metal plates with two Teflon O-rings placed on each side of the flat substrate. The O-rings placed on both sides of the flat substrate prevent leaking of the nanoparticle solution. A small hole drilled on the bottom alumina plate on each metal cup is to remain the pressure balance in sc- $\mathrm{CO}_{2}$ medium after the flat substrate is put between two Teflon $\mathrm{O}$ rings. Two aluminum heating block/reactor holders used to stabilize the high pressure reaction chamber and convey heat from the heating plate to the reaction chamber (Figure 46a). A type $\mathrm{J}$ thermocouple thermometer (Model 650, Omega Engineering Inc.) was connected to the chamber and indicated reaction temperatures. The apparatus loaded with a nanoparticle solution was placed in the high pressure reactor to remove the solvent resulting in film formation on the flat substrate surface. Because sc- $\mathrm{CO}_{2}$ has near zero surface tension, uniform nanoparticle films of different thickness can be formed using this setup [Figure 46d].

Distribution Statement A. Approved for public release; distribution unlimited. 


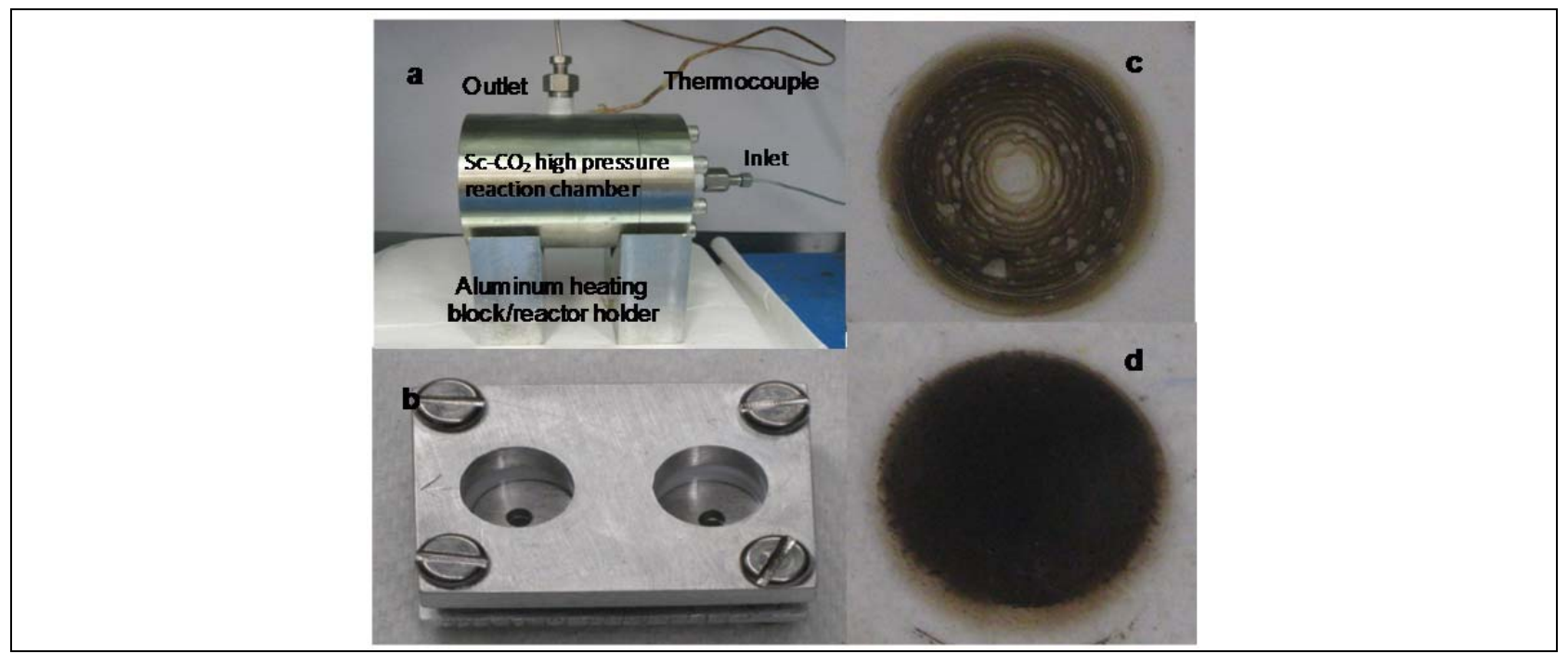

Figure 46. Sample forming apparatus for making nanoparticle films in sc-CO2. (a) sc$\mathrm{CO}_{2}$ high pressure reaction chamber, (b) an apparatus containing metal cups for $\mathrm{PbS}$ deposition, (c) PbS film deposited on glass using $\mathrm{SDM}$ and (d) sc- $\mathrm{CO}_{2}$ deposition methods. The lateral film density is about $0.49 \mathrm{mg} / \mathrm{cm}^{2}(\mathrm{~d})$.

To view surface morphology of the $\mathrm{PbS}$ nanoparticle films, we used chromium $(\mathrm{Cr})$ coated glass to prepare the films in $\mathrm{sc}-\mathrm{CO}_{2}$ following the same procedure described above for making $\mathrm{PbS}$ films on glass. The conductive nature of the $\mathrm{Cr}$ coated glass allows $\mathrm{SEM}$ imaging of the $\mathrm{PbS}$ nanoparticle films. A typical $\mathrm{SEM}$ picture of a $\mathrm{PbS}$ nanoparticle film prepared by the $\mathrm{sc}-\mathrm{CO}_{2}$ method is shown in Figure 47. The surface of the film is covered with orderly separated $\mathrm{PbS}$ nanoparticles.

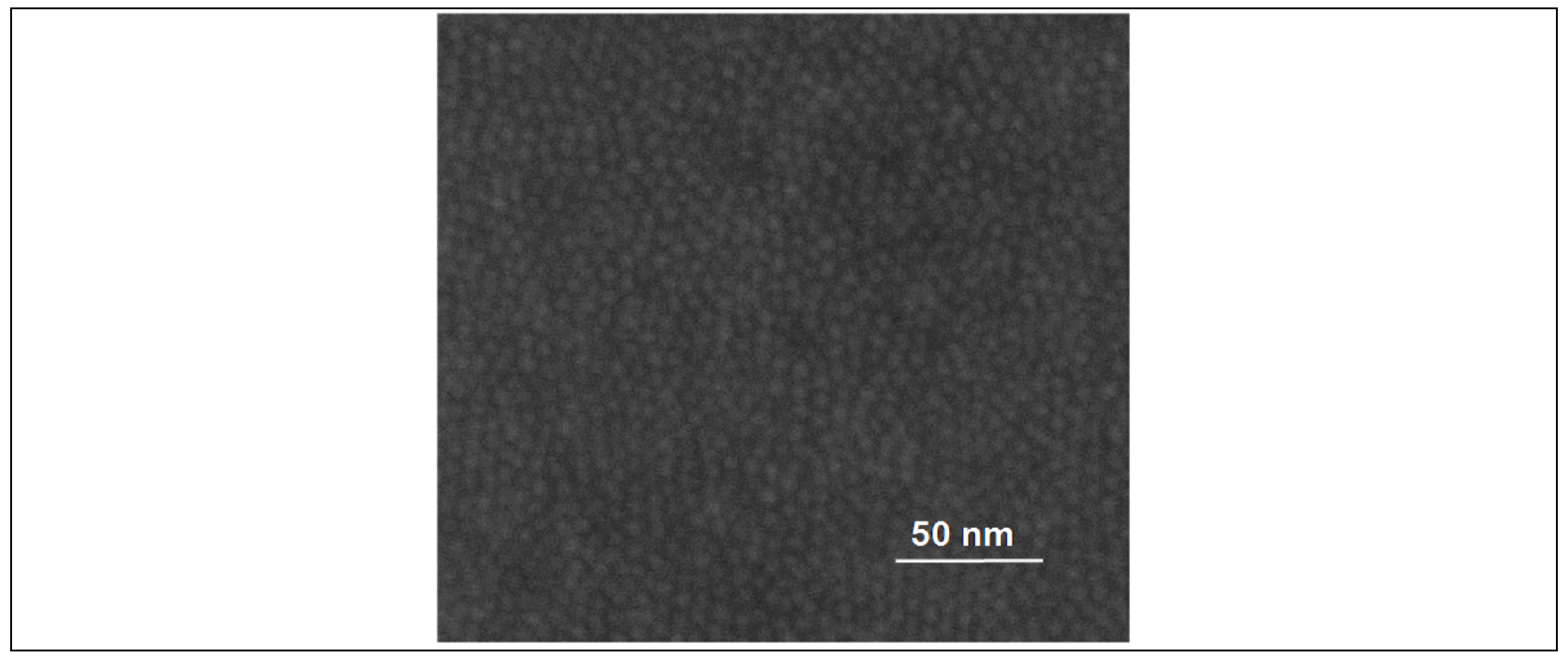

Figure 47. SEM image of surface of a $\mathrm{PbS}$ nanoparticle film prepared by the sc-CO deposition on a $\mathrm{Cr}$ coated glass. The lateral density of $\mathrm{PbS}$ on $\mathrm{Cr}$ coated glass is around 2.2 $\mathrm{mg} / \mathrm{cm}^{2}$

Distribution Statement A. Approved for public release; distribution unlimited. 


\section{Spectroscopic Studies of PbS QD Films Deposited on Glass}

We have demonstrated in the previous section that $\mathrm{sc}-\mathrm{CO}_{2}$ deposition is capable of making uniform arrays of $\mathrm{PbS}$ nanoparticles on flat substrate surfaces. The optical properties of the condensed metal sulfide nanoparticle arrays are largely unknown. Lead sulfide nanoparticle assemblies are of particular interest because they may lead to new optical materials for sensor applications in the infrared region. For this reason, we have developed methods of making uniform films of $\mathrm{PbS}$ QDs on different substrates in $\mathrm{sc}-\mathrm{CO}_{2}$ for spectroscopic studies. In our initial experiments, glass was selected as the insulate substrate for spectroscopic investigation of the $\mathrm{PbS}$ films.

Optical Microscope. An Axiotron optical microscope instrument was used to observe the surface morphology of the $\mathrm{PbS}$ nanoparticle films produced by a sc- $\mathrm{CO}_{2}$ deposition and by the conventional open air solvent deposition methods. The $\mathrm{PbS}$ nanoparticle film deposited on glass by sc- $\mathrm{CO}_{2}$ exhibits extremely uniform coverage on the glass substrate with a known amount of $\mathrm{PbS}$ nanoparticles in a fixed area for thick or for very thin films (Figures $48 \mathrm{~b}, 48 \mathrm{~d}$ ). The film produced by bench top SDM always shows irregular and ring-shaped coverage (Figures 48a, 48c). Comparing solvent and sc- $\mathrm{CO}_{2}$ depositions, the SDM process depends on surface tension effects on the interface of solvent and vapor, while in $\mathrm{sc}-\mathrm{CO}_{2}$ deposition, supercritical state eliminates the surface tension at the liquid/vapor interface and associated surface-wetting instabilities that have detrimental effects on the assembly of PbS nanoparticles into low-defect thin films $[2,26]$.

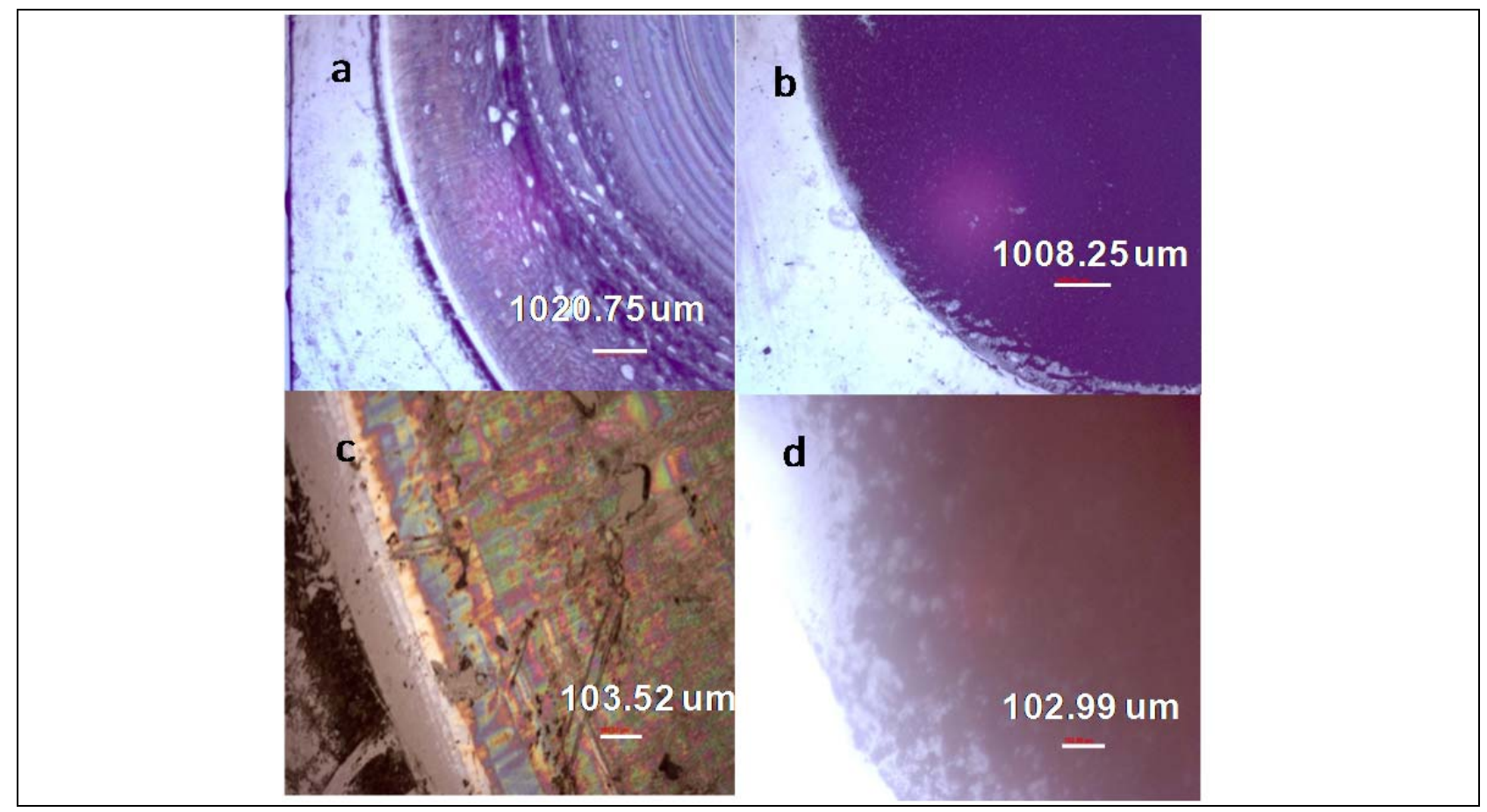

Figure 48. Microscope images of PbS QDs deposited on glass using solvent and sc-CO deposition methods in the same concentration $\left(\sim 2.2 \mathrm{mg} / \mathrm{cm}^{2}\right)$. (a) solvent deposition of PbS (scale: 1.25X ), (b) sc-CO $\mathrm{CO}_{2}$ deposition of $\mathrm{PbS}$ (scale:1.25X), (c) solvent deposition of PbS (scale: 10X), (d) sc-CO2 deposition of $\mathrm{PbS}$ (scale:10X).

Distribution Statement A. Approved for public release; distribution unlimited. 
UV-Vis-NIR. PbS solution containing different sizes $(2.3 \mathrm{~nm}$ and $4.8 \mathrm{~nm})$ in different concentrations were measured individually as shown in Figure 49. The maximum absorption wavelength in solutions for PbS QDs for the sizes of $2.3 \mathrm{~nm}$ and $4.8 \mathrm{~nm}$ remain almost unchanged with increases of concentrations and the maximum wavelength are around $900 \mathrm{~nm}$ (Figure 49, top) and $1350 \mathrm{~nm}$ (Figure 49, bottom), respectively.

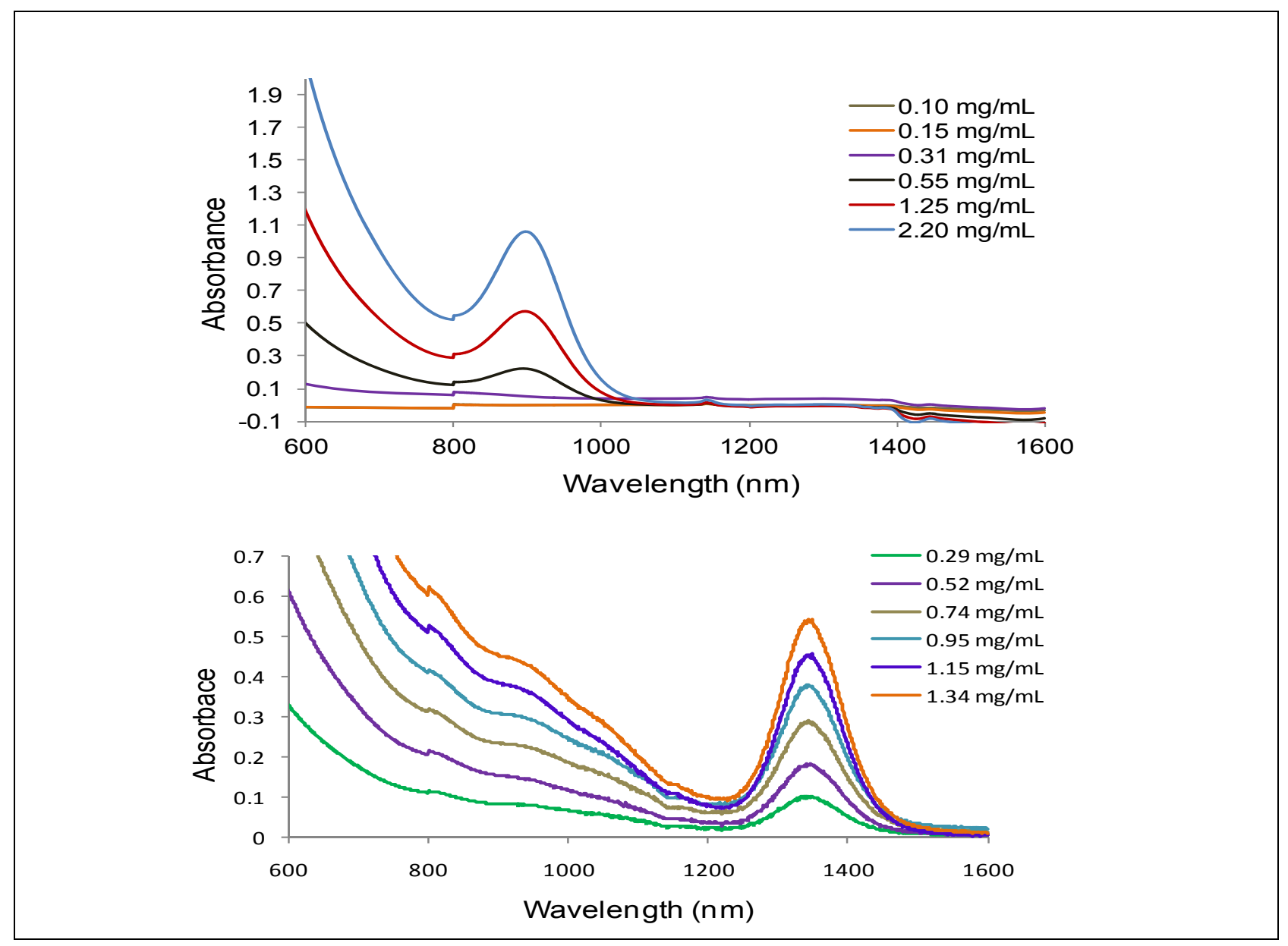

Figure 49. UV-Vis-NIR measurements of $\mathrm{PbS}(\operatorname{size}=2.3 \mathrm{~nm}$, top) and (size $=4.8 \mathrm{~nm}$, bottom)

Figure 50 reveals individual spectrum of PbS solution in different sizes of $2.7 \mathrm{~nm}$ and $4.8 \mathrm{~nm}$. The spectral peak ratio of $\mathrm{PbS}$ in Figure 50 for $2.7 \mathrm{~nm} / 4.8 \mathrm{~nm}$ is around 2:1. Using the same ratio, we mixed these two PbS solutions together. Figure 50 (bottom) show the spectra of two different sizes of $\mathrm{PbS} /$ toluene solution and $\mathrm{PbS}$ film fabricated using sc- $\mathrm{CO}_{2}$ deposition method. Based upon UV-Vis-NIR spectral information, the absorbance ratio of 2 peak intensities of $\mathrm{PbS} /$ toluene solution and the film remains similar. There is no obvious absorption wavelength change in the $\mathrm{PbS} /$ toluene solution and in the sc- $\mathrm{CO}_{2}$ depositions.

Distribution Statement A. Approved for public release; distribution unlimited. 

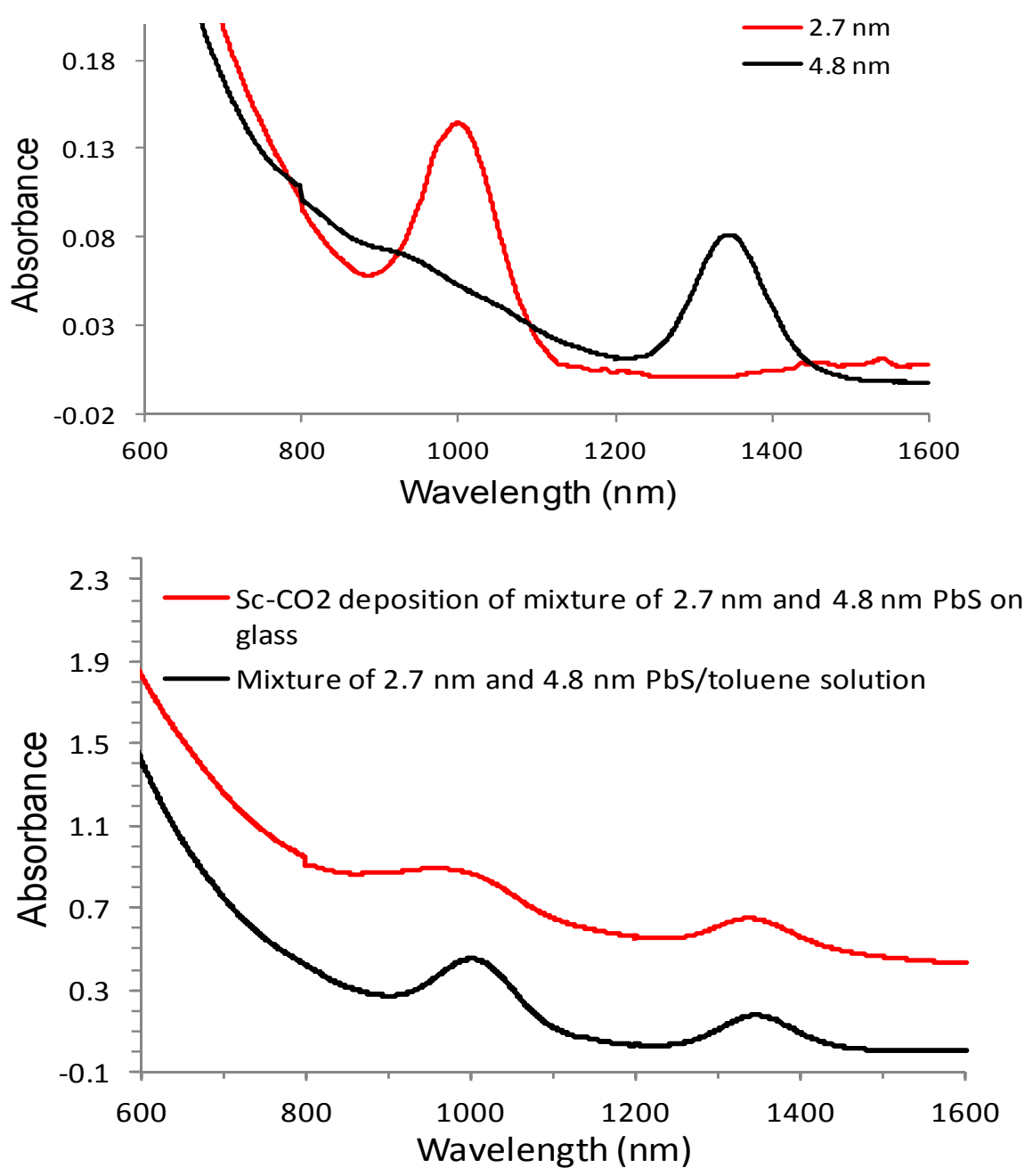

Figure 50. Absorption spectra of the PbS/toluene solutions in $2.7 \mathrm{~nm}$ and $4.8 \mathrm{~nm}$, measured individually (top). Absorption spectra of a mixture of $2.7 \mathrm{~nm}$ and $4.8 \mathrm{~nm} \mathrm{PbS} /$ toluene solution and the film prepared by $\mathrm{sc}-\mathrm{CO}_{2}$ evaporation method (bottom); film lateral density $\sim 2.2 \mathrm{mg} / \mathrm{cm}^{2}$.

Fluorescence. The possibility of energy transfer between $\mathrm{PbS}$ QDs of different sizes in the compact films formed by sc- $\mathrm{CO}_{2}$ deposition was evaluated. For this purpose, we prepared a mixed solution, which contained $2.3 \pm 0.3 \mathrm{~nm}$ and a $4.7 \pm 0.5 \mathrm{~nm} \mathrm{PbS}$ QDs in toluene. Using this solution, a film was prepared on glass in $\mathrm{sc}-\mathrm{CO}_{2}$ at $40{ }^{\circ} \mathrm{C}$ and a pressure $170 \mathrm{~atm}$. The fluorescence spectra of the mixed solution (bottom) and the film (top) are shown in Figure 51. Two distinct fluorescence peaks are visible. Regarding the solution, one is centered around 981 $\mathrm{nm}$ and the other $1374 \mathrm{~nm}$, corresponding to the emission wavelength of the $2.3 \mathrm{~nm}$ and the 4.7 nm QDs, respectively. The fluorescence peaks were normalized to illustrate the relative intensity ratio of the two peaks $(981 \mathrm{~nm} / 1374 \mathrm{~nm})$, which is approximately 8:9. We note that the film spectrum in the top part of Figure 51 reveals a shift of the peaks towards longer wavelengths. The significant observation in this experiment is that the ratio of the two peaks changed drastically to almost 1:10 for the film (Figure 51 top). The red-shift of the fluorescence

Distribution Statement A. Approved for public release; distribution unlimited. 
wavelength is still observed for each peak. The result strongly suggests occurrence of energy transfer from the smaller $\mathrm{PbS}$ particles to the larger $\mathrm{PbS}$ particles during the fluorescence process in the film. The energy transfer phenomenon between different sizes of $\mathrm{PbS}$ has been previously reported [30-32]. The kinetic rate of electronic energy transfer offers a pathway for the de-excitation of smaller particles (higher energy, electron donor) and excitation of larger particles (lower energy, electron acceptor) [30]. For mixed PbS QD film prepared by SDM under ambient pressure, changes in the relative fluorescence intensity are observed, but the values fluctuated depending on the location of the film measured. The uniformity of the $\mathrm{PbS}$ QD film prepared in sc- $\mathrm{CO}_{2}$ is apparently important, for studying optical properties of PbS QD films.

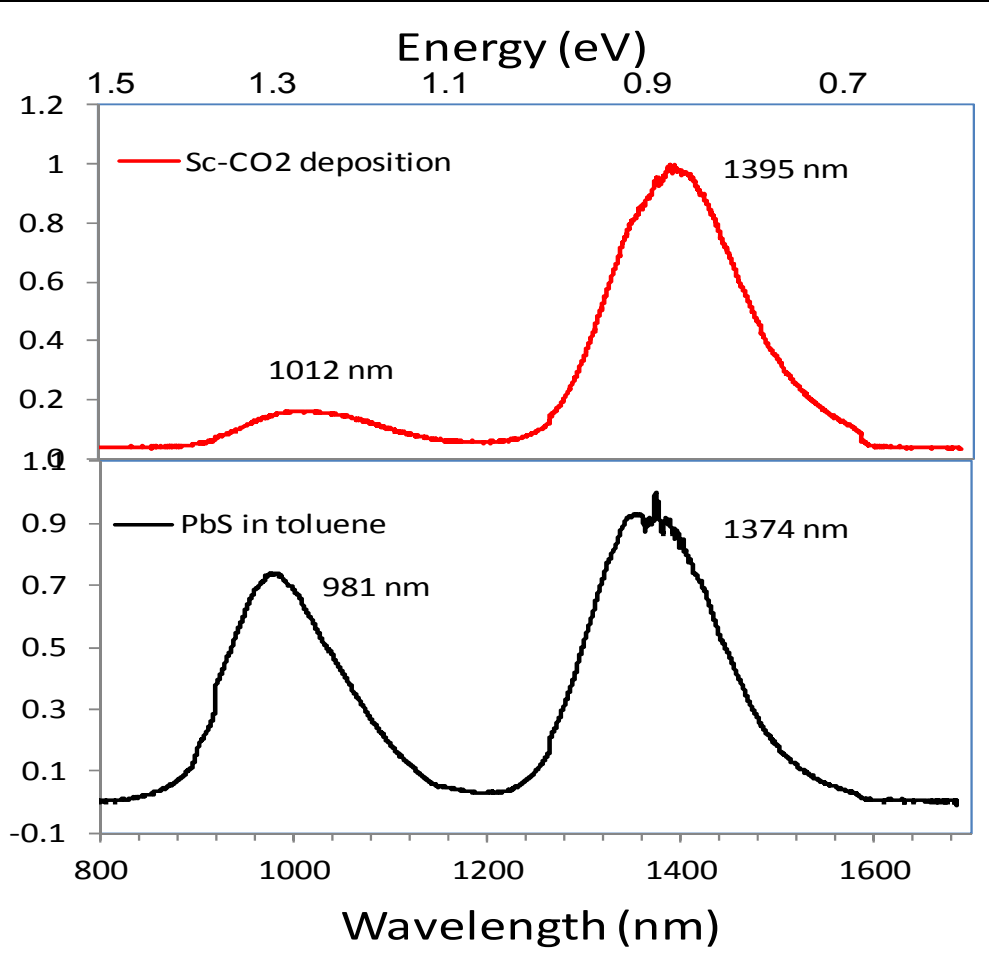

Figure 51. Fluorescence spectra of the mixed $\mathrm{PbS}$ nanoparticles in the original toluene solution (bottom) and in the film formed by sc- $\mathrm{CO}_{2}$ deposition of the solution (top), film lateral density $\sim 2.2 \mathrm{mg} / \mathrm{cm}^{2}$.

Photoluminescence. Figure 52 shows the PL at room temperature and cryogenic temperatures of samples formed on glass with the sc- $\mathrm{CO}_{2}$ and SDM methods. The sc- $\mathrm{CO}_{2}$ formed sample showed a shift towards lower energy with an enhanced PL intensity due to the more dense lateral QD distribution on the glass surface, causing energy transfer, in comparison to the sample deposited from the solution on the glass substrate. Especially at low temperature $5 \mathrm{~K}$, red-shift and enhanced emission intensity of $\mathrm{PbS}$ film deposited by sc- $\mathrm{CO}_{2}$ (close packed) was clearly observed. On the other hand, due to the denser arrangement of QDs in the sc- $\mathrm{CO}_{2}$ film, more QDs are excited by the laser beam and, as a consequence, the emission intensity is increased with respect to the sample formed with the solution deposition resulting in an inhomogeneous QD substrate coverage. Wise et al [32] used a theoretical equation to give an estimate of the distance - 5 or $6 \mathrm{~nm}$ - between the PbS particles. Under this condition, if the distance between

Distribution Statement A. Approved for public release; distribution unlimited. 
the particles is less than 5 or $6 \mathrm{~nm}$, energy transfer can occur in closed packed PbS QDs with oleic acid as capping agents on the surface [32], suggesting that the close-packed solid film have more possibilities to be red shifted, than in solution, or in solution deposited film. This red shift phenomenon is consistent with the information in the literature [30] reported previously, i.e., at low temperature, the PL of close packed QDs are more red-shifted comparing with that at room temperature.

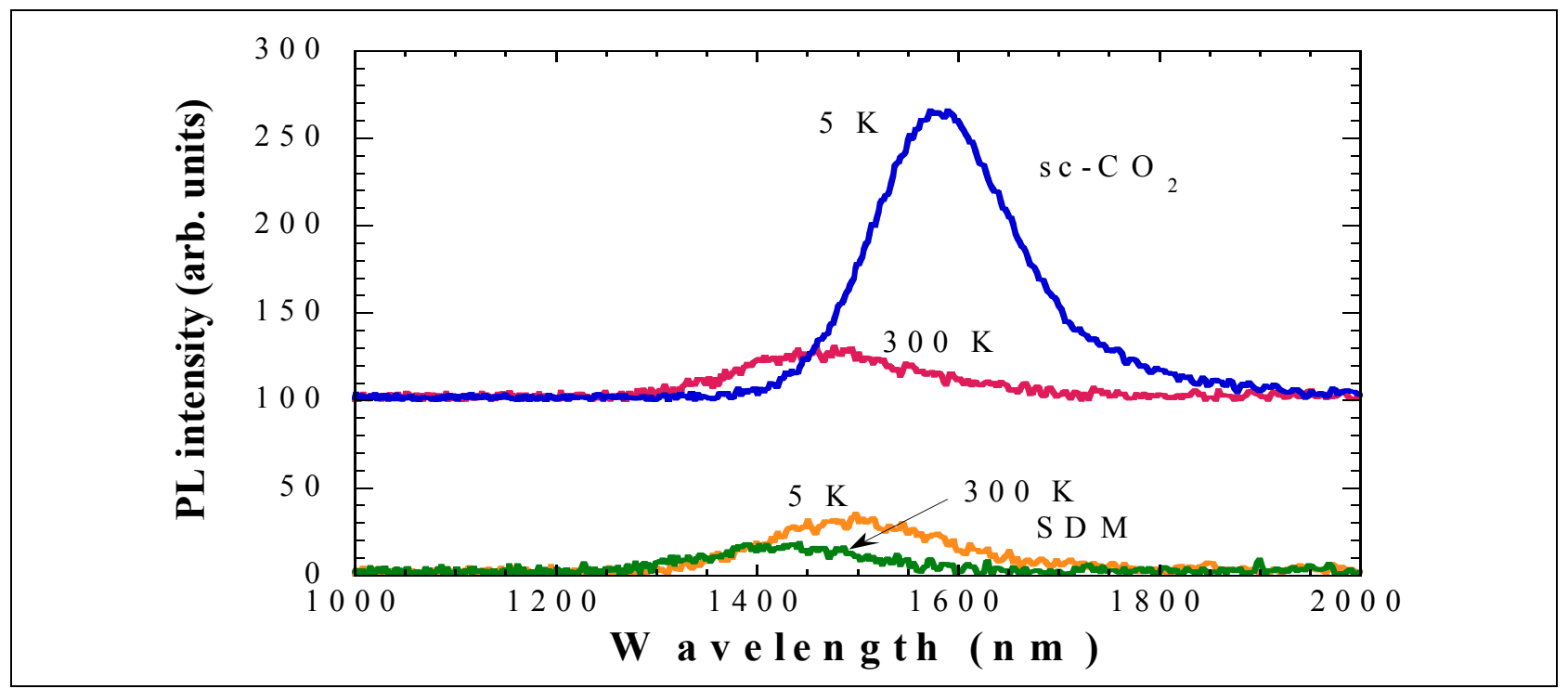

Figure 52. PL intensity vs. wavelength and energy at $5 \mathrm{~K}$ and $300 \mathrm{~K}$ of samples formed with sc- $\mathrm{CO}_{2}$ and SDM methods

Stability Studies of PbS QDs. The stability of the PbS QD solution was evaluated by PL. The unchanged PL intensity and PL peak position of newly prepared PbS films within the period of three months using the identical solution demonstrates its chemical stability. Figure 53 shows the $\mathrm{PL}$ measurements at cryogenic temperatures of $\mathrm{PbS}$ QDs deposited on $p$-type GaAs using the same $\mathrm{PbS}$ solution, before and after three months. It should be noted that the $\mathrm{PbS} /$ toluene solution used was stored at $4{ }^{\circ} \mathrm{C}$ in the dark in order to avoid photo-oxidation. Fairly time invariant emission properties of $\mathrm{PbS}$ QDs were reported also by other group when no photooxidation was enforced [23]. The PL peak intensities and PL peak positions of PbS films fabricated using sc- $\mathrm{CO}_{2}$ or SDM remained unchanged as well for the period of three months for the same film. TEM images of $\mathrm{PbS}$ nanoparticles using solvent and $\mathrm{sc}-\mathrm{CO}_{2}$ deposition methods [1] do not reveal conceivable changes after 16 months for the same PbS samples prepared on the TEM Cu grid, suggesting that the PbS nanoparticles are stable in the experimental time frame.

Distribution Statement A. Approved for public release; distribution unlimited. 


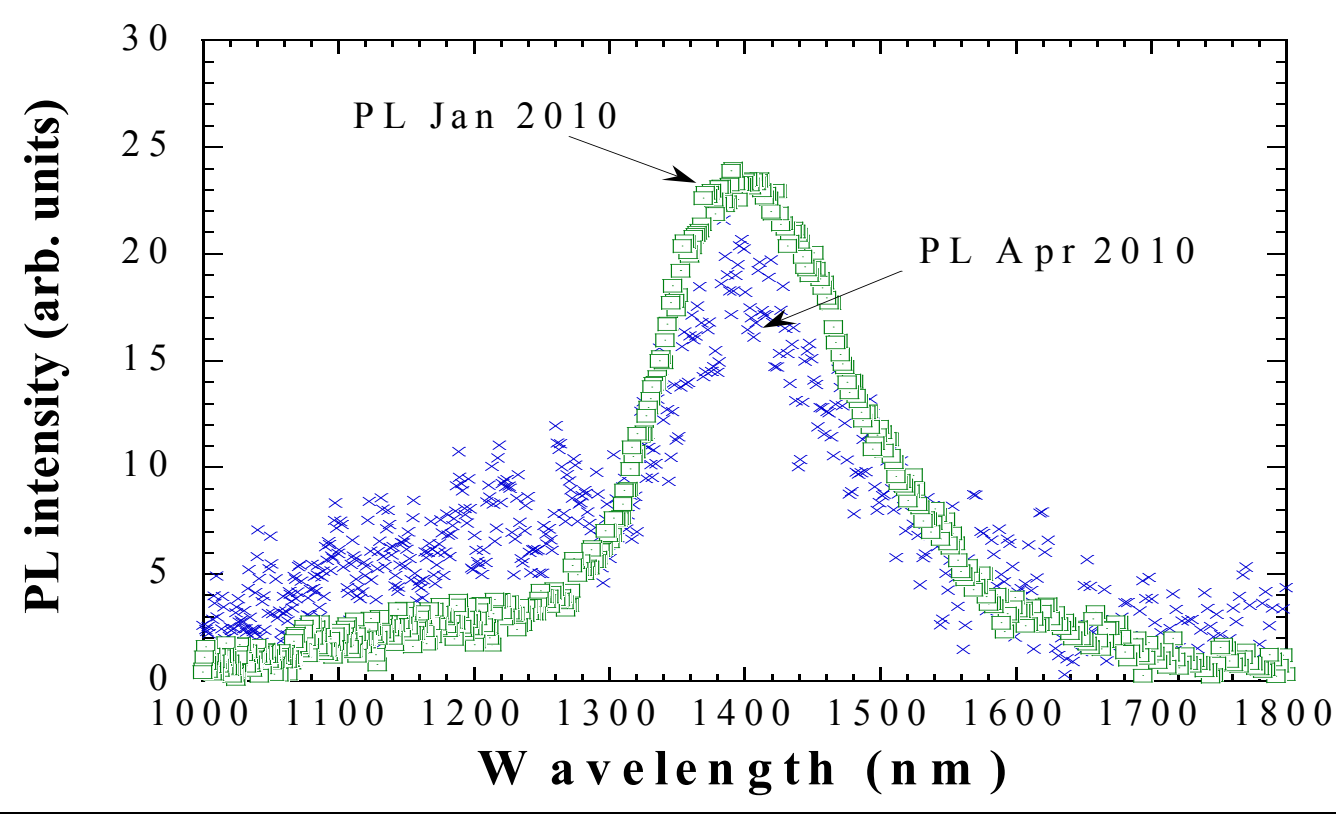

Figure 53. PL measurements of $\mathrm{PbS}$ deposited on $p$-type GaAs using the same $\mathrm{PbS}$ solution, before and after 3 months.

Figure 54 shows the XRD measurements for the PbS films prepared by a stock solution on glass, which was measured with PL initially $(\mathrm{t}=0)$; the $2^{\text {nd }}$ and $3^{\text {rd }}$ new PbS films were prepared on glass and measured 10 months and 23 months later, separately. PbS XRD pattern of $\mathrm{PbS}$ nanoparticles agrees very well with the standard reference data (JCPDS) for PbS galena structure, after nearly 2 years period of shelf life time. The galena structure is considered as a stable configuration. After nearly two years shelf life, the (111), (200), and (311) reflections are reduced, while on the other hand, (220) reflection is increased. Hence, according to Bragg's Law - $n \lambda=2 d \sin \theta$, where $\lambda$ is the x-ray wavelength, $\theta$ is the scattering angle, $n$ is the integer representing the order of the diffraction peak, and $d$ is the inter-plane distance - the lattice structure of the material did not change. The intensity fluctuations of the x-ray patterns might be caused by slightly different experimental conditions of the x-ray measurements.

Distribution Statement A. Approved for public release; distribution unlimited. 


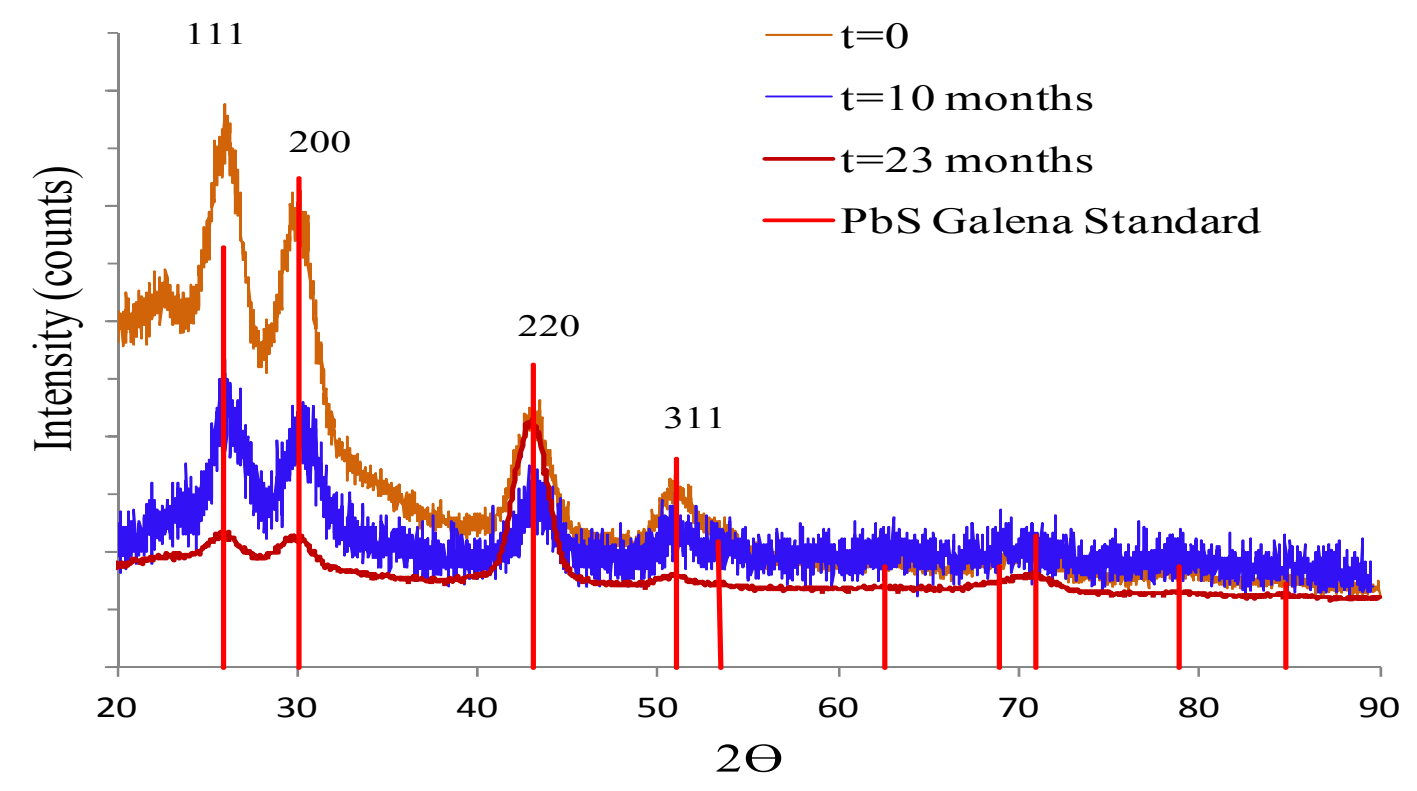

Figure 54. XRD measurements of $\mathrm{PbS}$ films deposited on glass, prepared initially $(\mathrm{t}=0)$, prepared 10 months and 23 months later.

The film thickness was measured with DekTak 6 M Stylus Profiler instrument. $3000 \mu \mathrm{m}$ scan length was used for a standard scan. The average scanned height corresponds to a thickness of $\mathrm{PbS}$ film, for instance, a film in Figure 46d, is around $1.0 \mu \mathrm{m}$. The films on the glass deposited by sc- $\mathrm{CO}_{2}$ are reasonably adhered to the glass surface and are not readily removed by an adhesive tape.

\section{Conclusions}

In this study, PbS QDs were synthesized with tunable sizes using sc- $\mathrm{CO}_{2}$ on various substrates. TEM studies indicate that the sc- $\mathrm{CO}_{2}$ method is capable of forming laterally homogeneous $\mathrm{PbS}$ QDs 2D arrays. On the other hand, bench top SDM results in non-uniform structures.

An apparatus for depositing $\mathrm{PbS}$ QDs on flat substrates employing sc- $\mathrm{CO}_{2}$ deposition method was designed in our lab. The PbS QDs deposited in this way have shown improved uniformity and areal coverage in comparison to SDM.

The absorbance maxima of the PbS QDs do not show an obvious dependence on the $\mathrm{PbS}$ concentrations both in $\mathrm{PbS} /$ toluene solution and $\mathrm{sc}-\mathrm{CO}_{2}$ fabricated film. The $\mathrm{PbS}$ sc- $\mathrm{CO}_{2}$ samples deposited on glass show clearly resolved absorbance features in the Vis-NIR region, and strong intensity in fluorescence and PL, which are attributed to the narrow particle size distribution and homogeneous particle morphology. Energy transfer occurs in close packed $\mathrm{PbS}$ QDs in the emission processes of fluorescence and PL. The difference of the emission properties of samples formed with sc- $\mathrm{CO}_{2}$ deposition method and SDM is visualized by PL measurements. The temporal invariance of the structural and optical properties of the formed QD arrays is appears to be sufficient for technological applications.

Distribution Statement A. Approved for public release; distribution unlimited. 


\section{References}

[1] J. S. Wang, A. B. Smetana, J. J. Boeckl, G. J. Brown, C. M. Wai, Langmuir, 2010, 26 (2), 117-1123.

[2] A. B. Smetana, J. S. Wang, J.J. Boeck1, G. J. Brown, C. M. Wai, J. Phys. Chem. C, 2008, 112 (7), 2294-2297.

[3] W. Jang, D. Kim, J. Kim, B. K. Min, J. Kim, K. Yoo, Chem. Mater., 2010, 22 (15), 4350-4352.

[4] A Behnam, J. L. Johnson, Y. An, A. Biswas, and A. Ural, ACS Nano, 2011, 5 (3), $1617-$ 1622.

[5] P. X. Gao, Y. Ding and Z..L. Wang,Nano Lett., 2009, 9 (1), 137-143.

[6] M. Navaneethan, K.D. Nisha, S. Ponnusamy, C. Muthamizhchelvan, Materials Chemistry and Physics, 2009, 117(2-3), 443-447.

[7] X. Zhao, I. Gorelikov, S.Musikhin, S. Cauchi,V. Sukhovatkin, E. H. Sargent, E. Kumacheva, Langmuir, 2005, 21, 1086-1090.

[8] S. S. Brown, A. J. Rondinone, S. Dai, 2007, ACS Symposium Series 980, American Chemical Society, Washington, DC, Chapter 8, pp 117-129.

[9] S. Mubeen, T. Zhang, B. Yoo, M. A. Deshusses, N. V. Myung, J. Phys. Chem. C, 2007, 111 (17), 6321-6327.

[10] R. Nagarajan, 2008, ACS Symposium Series 996, American Chemical Society, Washington, DC, Chapter 1, pp 2-14.

[11] X. F. Wu, H. Y. Song, J. M. Yoon, Y. T. Yu, Y. F. Chen, Langmuir, 2009, 25 (11), 6438-6447.

[12] G. K. Kouassi, J. Irudayaraj, Anal. Chem., 2006, 78 (10), 3234-3241.

[13] Murray, C. B.; Norris, D. J.; Bawendi, M. G. J. Am. Chem. Soc. 1993, 115, 8706.

[14] (a)Alivisatos, A. P. Science 1996, 271, 933. (b) Bruchez, M.; Moronne, M.; Gin, P.; Weiss, S.; Alivisatos, A. P. Science 1998, 281, 2013.

[15] Li, J. J.; Wang Y. A.; Guo, W.; Keay, J. C.; Mishima, T. D.; Johnson, M. B.; Peng, X. J. Am. Chem. Soc. 2003, 125, 12567.

[16] Chan, W. C. W.; Nie, S. M. Science 1998, 281, 2016.

[17] (a) Huynh, W. U.; Dittmer, J. J.; Alivisatos, A. P. Science 2002, 295, 2425. (b) Klimov, V. I.; Mikhailovsky, A. A.; Xu, S.; Malko, A.; Hollingsworth, J. A.; Leatherdale, C. A.; Eisler, H.-J.; Bawendi, M. G. Science 2000, 290, 314.

[18] Zeng, Z. H.; Wang, S. H.; Yang, S. H. Chem. Mater. 1999, 11, 3365.

[19] Ding, T.; Zhang, J. R.; Long, S.; Zhu, J. J. Microelectron. Eng. 2003, 66, 46.

[20] Smetana, A. B.; Wang, J. S.; Boeckl, J. J.; Brown, G.J.; Wai, C. M. Langmuir 2007, 23, 10429.

[21] M. A. Hines, G. D. Scholes, Adv. Mater. 2003, 15(21), 1844-1849

[22] J. H. Warner, E. Thomsen, A. R. Watt, N. R. Heckenberg, H. Rubinsztein-Dunlop, Nanotechnology, 16 (2005), 175-179

[23 ] K. A. Abel, J. Shan, J. C. Boyer, F. Harris, F. C. J. M. van Veggel, Chem. Mater. 2008, 20, 3794-3796

[24] Wang, Y.; Suna, A.; Mahler, W.; Kasowski, R. J. Chem. Phys. 1987, 87, 7315.

Distribution Statement A. Approved for public release; distribution unlimited. 
Y. Nosaka, J. Phys Chem. 1991, 95, 5054

[26] Liu, J.; Anand, M.; Roberts, C. B. Langmuir, 2006, 22(9), 3964

[27] McLeod, M. C.; Kitchens, C.L.; Roberts, C.B. Langmuir 2005, 21, 2414.

[28] Ohara, P.C.; Gelbart, W.M. Langmuir 1998, 14, 3418.

[29] Korgel, B. A.; Fitzmaurice, D. Phys. Rev. Lett. 1998, 80, 3531.

[30] C. R. Kagan, C. B. Murray, M.G. Bawendi, Phys. Review B 1996, 54 (12) , 8633.

[31] C.R. Kagan, C.B. Murray, M. Nirmal, M.G. Bawendi, Phys. Review Lett. 1996, 76(9), 1517.

[32] Clark, S.W.; Harbold, J.M.; Wise, F.W. J. Phys. Chem. C 2007, 11 1(20), 7302.

\title{
3.4 Lead Sulfide Quantum Dot Synthesis, Deposition, and Temperature Dependence Studies of the Stokes Shift
}

J. S. Wang, B. Ullrich, and G. J. Brown

Air Force Research Laboratory, Materials \& Manufacturing Directorate, Wright Patterson AFB, OH 45433-7707, USA

\begin{abstract}
We investigated the temperature dependence of the Stokes shift of PbS quantum dots (diameter $4.7 \mathrm{~nm}$ ) deposited from solution on glass using a specially designed apparatus. By measuring the thermal alteration of the optical absorbance and photoluminescence in the range of $5 \mathrm{~K}-300 \mathrm{~K}$, we demonstrate that the Stokes shift shrinks from $135 \mathrm{meV}$ at $5 \mathrm{~K}$ to $62 \mathrm{meV}$ at $300 \mathrm{~K}$. Extrapolation of the data presented predict an elimination temperature of the Stokes shift of about $460 \mathrm{~K}$, corresponding to the thermal energy of the sum of prominent PbS phonon energies.
\end{abstract}

\section{INTRODUCTION}

Lead Sulfide quantum dots (QDs) have attracted considerable attention owing to the narrow direct band gap $(0.41 \mathrm{eV})$ and large exciton Bohr radius, providing an excellent system for studying quantum confinement effects [1]. However, despite these extensive studies there are still opportunities to further understanding of the physics of the optical properties associated with the confined states. One such topic is the understanding of the origin of the Stokes shift between the peaks of photoluminescence (PL) and optical absorbance (OA) of PbS QDs. The PL of PbS QDs has been studied intensively during the previous years, where particular attention was paid to the thermal shift of the emission spectra [2-5]. In addition, some of the works compare both the room temperature absorption $[3,5,6]$ and the absorption at cryogenic temperatures $[5,7,8]$ with the PL spectra and show a redshift of the PL with respect to the absorption edge. This separation is referred to as the Stokes shift and is usually attributed to multi-phonon driven energy relaxations into the dark exciton state before luminescent transitions occur [8]. The influence of the size [7,9] and stoichiometry [9] on the Stokes shift was studied for PbS QDs but not its temperature dependence $\mathrm{S}(T)$. The analysis of $\mathrm{S}(T)$, which is of considerable importance for further understanding of the PL transition and the realization of technological applications including anti-Stokes cooling [10], is presented in this paper.

\section{EXPERIMENTAL DETAILS}

The PbS QDs used in this study were synthesized in organic solvents, with oleic acid as a capping agent, based on a method published in the literature [11]. The synthesis starts with the

Distribution Statement A. Approved for public release; distribution unlimited. 
reaction of $\mathrm{PbO}$ with oleic acid to form lead oleate by heating oleic acid with $\mathrm{PbO}$ in a round bottom flask under Ar atmosphere while stirring. The temperature was kept at $150^{\circ} \mathrm{C}$ for $1 \mathrm{hr}$. After this time - still keeping the temperature at $150^{\circ} \mathrm{C}$ - a sulfide source, bis(trimethylsilyl) sulfide, was injected into the system and the reaction mixture was allowed to ripen for $1 \mathrm{hr}$ at $100^{\circ} \mathrm{C}$. The sample was then washed with methanol, which removed the excesses of oleic acid and octadecene. The QDs were dried by a stream of $\mathrm{N}_{2}$ and then dissolved in toluene. The size of the $\mathrm{PbS}$ QDs can be altered by changing the oleic acid/octadecene ratios and also can be controlled by changing injection and growth temperatures.

Using this process, PbS QDs of different sizes have been selectively synthesized and deposited utilizing different deposition approaches in order to create thin films and close-packed arrays. For this study, PbS QDs with a diameter of $4.7 \mathrm{~nm}$ were used, motivated by the room temperature emission wavelengths in the range $1.3 \mu \mathrm{m}-1.55 \mu \mathrm{m}$, which is important for telecommunication applications. We chose to investigate the PL properties of the PbS QD film formed on glass employing solvent evaporation deposition to avoid any interference of the substrate with the optical properties. The homemade apparatus for deposition consists of two aluminum plates, each with a circular 0.5-inch opening in the center (Figure 55, left). A piece of glass is inserted between the plates with two Teflon o-rings placed on each side of the glass substrate in order to prevent leaking of the QD solution. The sample was formed by mixing 70 $\mu \mathrm{L}$ of PbS QDs (40 mg/mL, diameter $4.7 \pm 0.5 \mathrm{~nm}$ ) with $200 \mu \mathrm{L}$ toluene solvent. After keeping the $\mathrm{PbS}$ toluene solution in the dark for approximately $30 \mathrm{~min}$, the solvent was evaporated and the dry $\mathrm{PbS} /$ glass sample was removed from the holder, as shown in Figure 55, right.

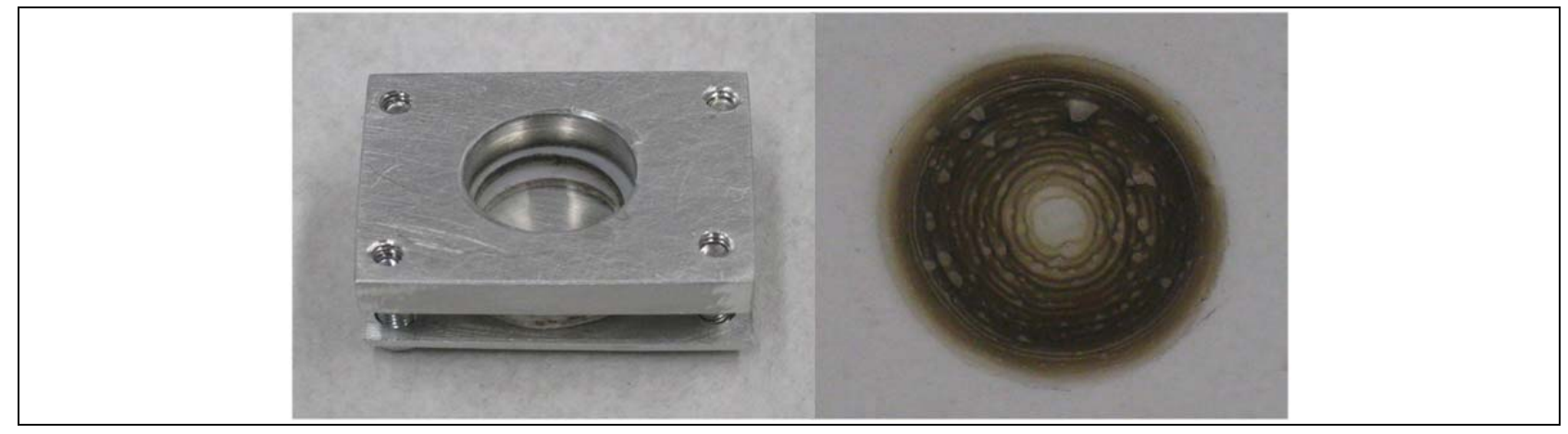

Figure 55. Homemade apparatus for solvent deposition (left) and the formed PbS QDs on glass (right).

The PL and OA measurements were carried out with BOMEM Fourier transform spectrometers. The PL was excited with the continuous wave (cw) $532 \mathrm{~nm}$ emission of a solid-state laser keeping the intensity at about $5 \mathrm{~W} / \mathrm{cm}^{2}$. The sample emission was detected with an InSb detector and the sample temperature was controlled by an optical closed-cycle helium cryostat. The OA spectrum of the sample was determined via the transmittance, using the internal globar source and the InSb detector of a second BOMEM spectrometer. The OA was calculated using $\log \left(I_{0} / I\right)$, where $I_{0}$ is the impinging light intensity and $I$ is the transmitted light intensity. The sample temperature was again regulated with an optical closed-cycle helium cryostat.

Distribution Statement A. Approved for public release; distribution unlimited. 


\section{DISCUSSION}

Figure 56 shows the PL and OA spectra measured at $5 \mathrm{~K}$ for the thin-film of PbS QDs on glass. A clear Stokes shift of $135 \mathrm{meV}$ is observed. This is comparable to the $150 \mathrm{meV}$ Stokes shift at $10 \mathrm{~K}$ reported by Zhang and Jiang for $4 \mathrm{~nm} \mathrm{PbS}$ QDs on sapphire [7]. At room temperature our measured Stokes shift was only $62 \mathrm{meV}$. In Figure 57 the measured PL and OA peak positions are plotted over the entire range from $5 \mathrm{~K}$ to $300 \mathrm{~K}$. Clearly, the separation between the PL and OA peak positions decreases as the sample temperature is increased. In order to understand the origin of the temperature dependence of the Stokes shift, we first consider the temperature dependence of the OA and PL peaks separately.

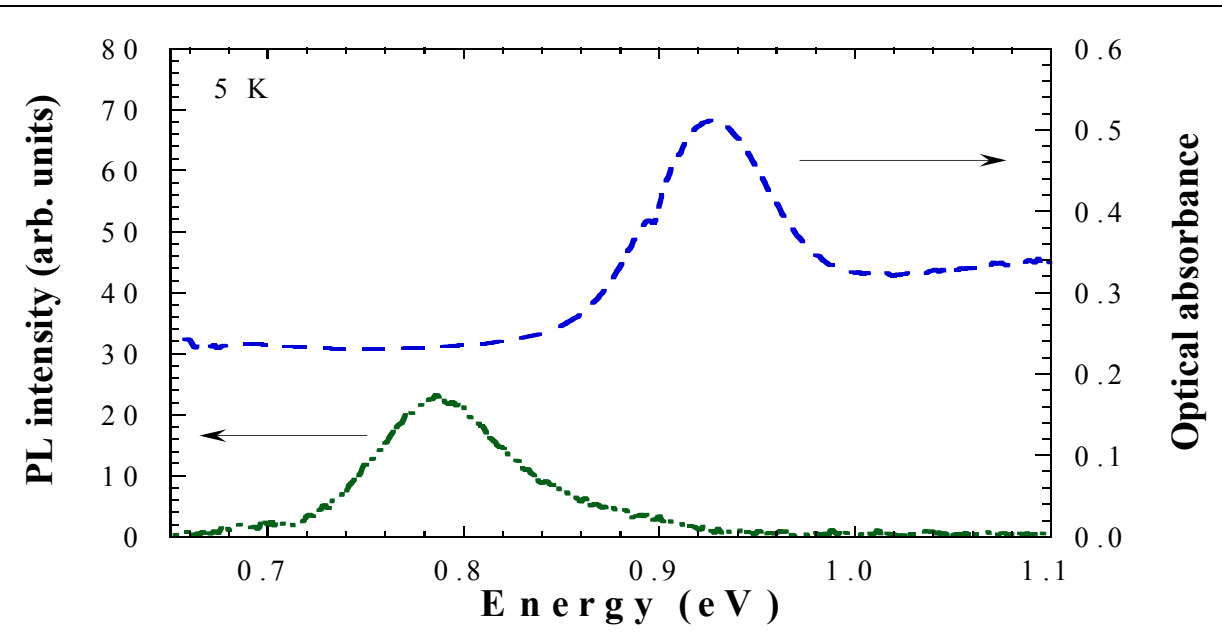

Figure 56. PL and OA spectra measured at $5 \mathrm{~K}$.

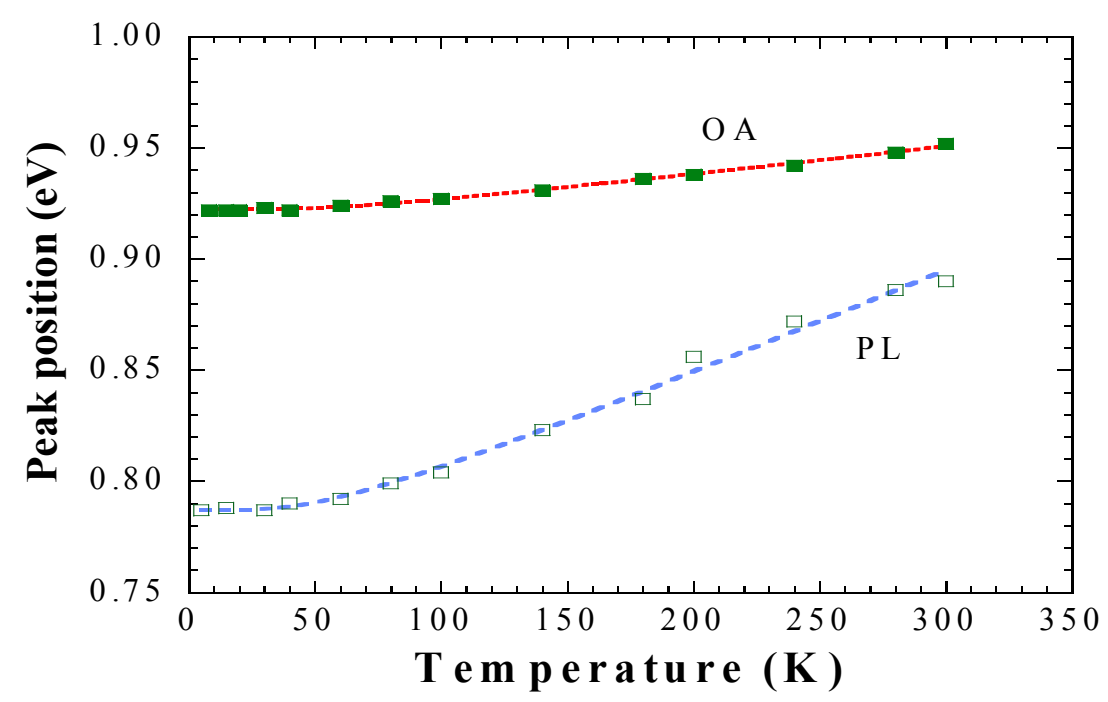

Figure 57. The symbols show the measured thermal shift of the PL ( $\square$ ) and OA (घ) peaks. The dashed lines are the fits using Equation (22).

Distribution Statement A. Approved for public release; distribution unlimited. 
Table 8. Fitting parameters for the fits in Figure 57 done with Equation (22)

\begin{tabular}{|c|c|c|c|}
\hline Measurement & $\left.\boldsymbol{E}_{\mathbf{g}}(\mathbf{0}) \mathbf{( e V}\right)$ & $\boldsymbol{A} \mathbf{( m e V )}$ & $\left\langle\boldsymbol{E}_{\mathbf{p}}>\mathbf{( m e V}\right)$ \\
\hline OA & 0.922 & 23.4 & 15.5 \\
\hline PL & 0.787 & 73.9 & 13.5 \\
\hline
\end{tabular}

For the current work, the thermal band gap shift $E_{\mathrm{g}}(T)$ is the key parameter. We use for this purpose the expression of Fan [12],

$$
E_{\mathrm{g}}(T)=E_{\mathrm{g}}(0)+A /\left\{\exp \left(<E_{\mathrm{p}}>/ k T\right)-1\right\},
$$

where $E_{\mathrm{g}}(0)$ is the low temperature limit of the band gap energy, $A$ is the Fan parameter, which depends on the microscopic material properties, $\left\langle E_{\mathrm{p}}>\right.$ is the average phonon energy responsible for the band gap variation, and $k T$ is the thermal energy. The two sets of data were fitted using Equation (22) but required different fitting parameters. The fitting parameters are displayed in Table 8 . There is good agreement between the averaged $\left\langle E_{\mathrm{p}}\right\rangle(=14.5 \mathrm{meV})$ of these two fits and the value for bulk $\mathrm{PbS}(14 \mathrm{meV})[3,13]$. However, the Fan parameter is much larger for the PL fit. This highlights a difference between the nature of the states involved in the PL and OA transitions.

The difference between the $\mathrm{AO}$ and PL peak energies arises from the different processes involved in the two transitions: The OA process involves excitation from the hole confined state (h1) to the electron confined state (e1), while the PL process involves recombination of an excited electron with a lower energy hole. If the holes were in the h1 state then the emitted photon energy would coincide with the absorption energy. For a Stokes shift to occur, the e $\mathrm{e}^{-}-\mathrm{h}$ recombination must take place at a lower energy state. For PbS QDs capped with oleic acid, the literature indicates that the PL involves a trap state, which is an acceptor state on the QD surface caused by unpassivated sulfur [14,15]. For 1-2 $\mathrm{nm}$ PbS QDs, Fernée et al. reported that the trap state was at least $375 \mathrm{meV}$ below the bulk $\mathrm{PbS}$ valence band edge [14]. The surface trap energy is known to vary with QD sizes [15]. For our $4.7 \mathrm{~nm}$ QDs the Stokes shift can be fitted assuming hole trap states around $200 \mathrm{meV}$ below the bulk valence band as outlined in Figure 58.

Distribution Statement A. Approved for public release; distribution unlimited. 


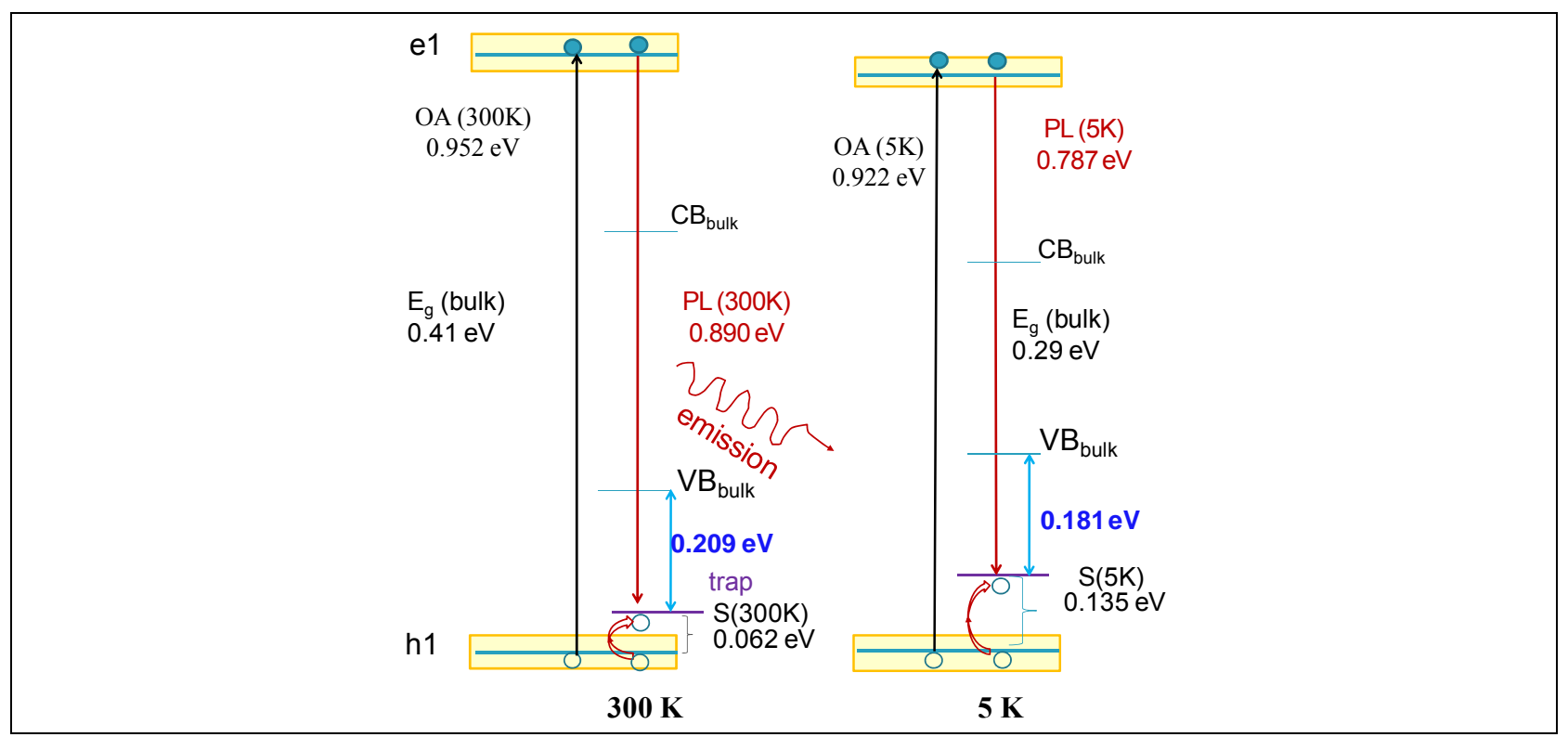

Figure 58. Schematic of the $\mathrm{OA}$ and PL transitions with hole trap states tied to the bulk $\mathrm{PbS}$ valence band edge. The shift of the trap state with temperature results in the temperature dependence of the Stokes shift.

There are reports of very small Stokes shifts $(\sim 10 \mathrm{meV})$ at room temperature for well-passivated $\mathrm{PbS}$, indicating that the surface states are an important factor in the measured Stokes shift $[11,16]$.

In order to better visualize the temperature dependence of $S(T)$, the energy difference between the OA and PL peaks is plotted versus $T$ in Figure 59. Considering the good fit with Equation (22) for the PL and OA data in Figure 57, the formula fitting $\mathrm{S}(T)$ ought to be an expression like Equation (22). We advocate the following expression for $\mathrm{S}(T)$ :

$$
\mathrm{S}(T)=\mathrm{S}(0)-C /\left\{\exp \left(<E_{\mathrm{p}}>/ k T\right)-1\right\}
$$

where, $\mathrm{S}(0)$ is the Stokes shift at temperatures approaching $0 \mathrm{~K}, C$ is a constant, and $\left\langle E_{\mathrm{p}}>\right.$ is again the average phonon energy.

The dotted line in Figure 59 shows the fit of the data with Equation (23) using the fitting parameters of $\mathrm{S}(0)=0.135 \mathrm{eV},<E_{\mathrm{p}}>=14.5 \mathrm{meV}$ and $C=60.2 \mathrm{meV}$. At low temperatures $<100 \mathrm{~K}$, the curve fits the data better than at higher temperatures suggesting that at cryogenic temperatures the average phonon energy is responsible for the thermal variation of absorption and luminescent transitions. At higher temperatures, however, multi-phonon processes contribute to $\mathrm{S}(T)$.

Distribution Statement A. Approved for public release; distribution unlimited. 


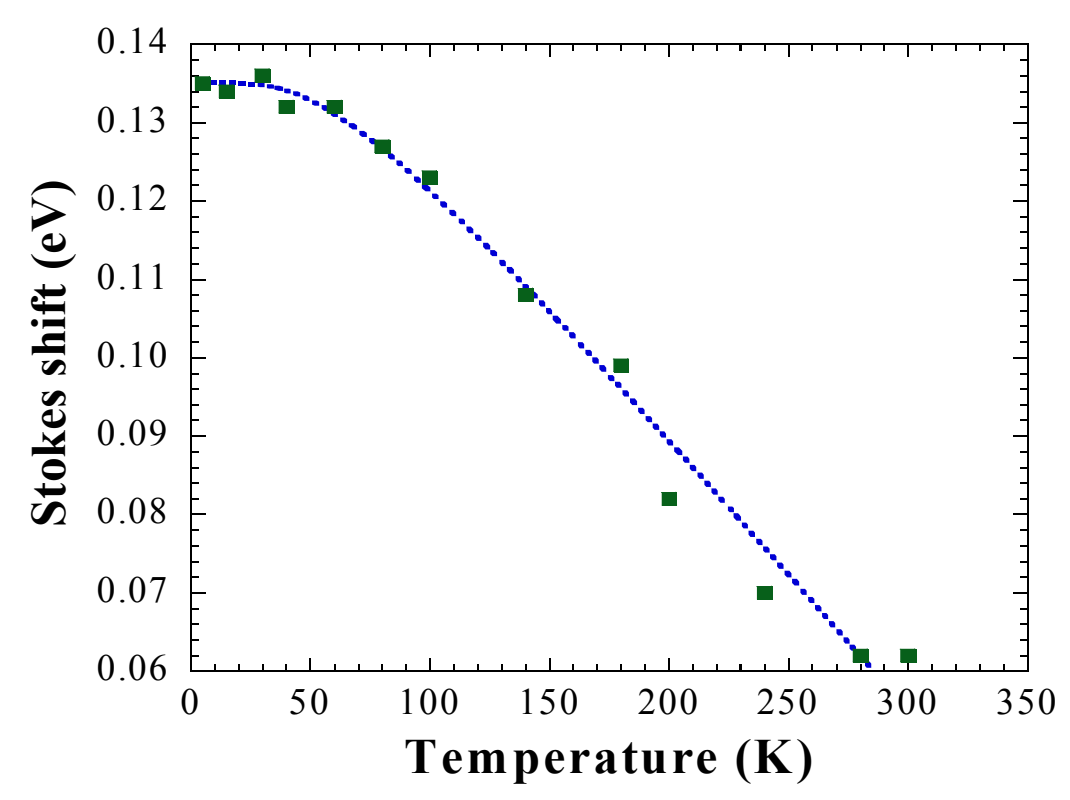

Figure 59. The difference between the energies of the OA and PL peaks in Figure 57. The dotted line is the fit using $\left\langle E_{\mathrm{p}}\right\rangle=14.5 \mathrm{meV}, \mathrm{S}(0)=135 \mathrm{meV}$, and $C=60.2 \mathrm{meV}$ in Equation (23).

Equation 23 allows the calculation of the critical temperature $T_{\mathrm{c}}$ where $\mathrm{S}\left(T_{\mathrm{c}}\right)=0$, i.e.,

$$
T_{\mathrm{c}}=\frac{<E_{\mathrm{p}}>}{k \ln \left(\frac{C}{\mathrm{~S}(0)}+1\right)},
$$

where $k=8.62 \times 10^{-5} \mathrm{eV} / \mathrm{K}$, and we find that $T=457 \mathrm{~K}$, corresponding to $k T=39.4 \mathrm{meV}$. It is evident that more work needs to be done in order to achieve a clear understanding of the reduction of the Stokes shift with increasing temperature but we note that this number matches quite well the sum of $\left\langle E_{\mathrm{p}}\right\rangle=14.5 \mathrm{meV}$ and the phonon energy $E_{\mathrm{LO}}(\Gamma)=26 \mathrm{meV}$ [2]. We have just begun with the systematic analysis of PbS QDs prepared with different methods on various substrates and will carry out further experiments at elevated temperatures.

In conclusion, we studied the temperature dependence of the Stokes shift of PbS QDs. The thermally induced shifts of the OA and PL peaks were fit with the Fan equation. Similarly, the energy difference between these two peaks can be equivalently well fitted with an equation of identical structure. At temperatures $\leq 100 \mathrm{~K}, \mathrm{~S}(T)$ is related to the bulk average phonon energy $(\sim 14 \mathrm{meV})$, while a trap model was required to explain the observed values of $\mathrm{S}(T)$. Based on data collected up to $300 \mathrm{~K}$, the fitting of the thermal Stokes shift variation predicts the existence of a critical temperature around $460 \mathrm{~K}$, where no Stokes shift takes place, corresponding to the sum of two prominent phonon energies.

Distribution Statement A. Approved for public release; distribution unlimited. 


\title{
REFERENCES:
}

1. J. J. Peterson and T. D. Krauss, Nano Lett. 6, 510 (2006).

2. L. Turyanska, A. Patanè, M. Henini, B. Hennequin and N. R. Thomas, Appl. Phys. Lett. 90, 101913 (2007).

3. M. S. Gaponenko, A. A. Lutich, N. A. Tolstik, A. A. Onushchenko, A. M. Malyarevich, E. P. Petrov and K. V. Yumashev, Phys. Rev. B 82, 125320 (2010).

4. B. Ullrich, X. Y. Xiao, and G. J. Brown, J. Appl. Phys. 108, 013525 (2010).

5. B. Ullrich, J. S. Wang, and G. J. Brown, Appl. Phys. Lett. 99, 081901 (2011).

6. C. Liu, K. Kwon, and J. Heo, J. Non-Cryst. Solids 355, 1880 (2009).

7. J. Zhang, X. Jiang, J. Phys. Chem. B 112, 9557 (2008).

8. J. Zhang and X. Jiang, Appl. Phys. Lett. 92,141108 (2008).

9. N. O. Dantas, P. M. N. de Paula, R. S. Silva, V. López-Richard, G. E. Marques, J. Appl. Phys. 109, 024308 (2011).

10. Y. P. Rakovich, J. F. Donegan, M. I. Vasilevskiy, A. L. Rogach, Phys. Stat. Sol. A 206, 2497 (2009).

11. M. A. Hines and G. D. Scholes, Adv. Mater. 15(21), 1844, (2003).

12. I. A. Vainshtein, A. F. Zatsepin, and V. S. Kortov, Phys. Solid State 41, 907 (1999).

13. O. Madelung, Semiconductors: Data Handbook, $3^{\text {rd }}$ ed. (Springer, Berlin, 2004).

14. M. J. Fernée, E. Thomsen, P. Jensen, and H. Rubinsztein-Dunlop, Nanotech 17, 956 (2008).

15. A. Lobo, T. Muller, M. Nagel, H. Borchert, S.G. Hickey, and H. Weller, J. Phys. Chem. C 109, 17422 (2005).

16. K. A. Abel, J. Shan, J.-C. Boyer, F. Harris, and F. C. J. M. van Veggel, Chem. Mater. 20, 3794 (2008).

\subsection{Emission of Precipitation Deposited PbS Quantum Dots on Polyethylene Terephthalate}

Bruno Ullrich", Andrew R. Markelonis, Joanna S. Wang, and Gail J. Brown

Air Force Research Laboratory, Materials \& Manufacturing Directorate, Wright Patterson AFB, OH 45433-7707, USA

\begin{abstract}
Centrifuge enforced precipitation was used to disperse PbS quantum dots (diameter $4.7 \mathrm{~nm}$ ) on polyethylene terephthalate. By employing double frequency Fourier transform spectroscopy, we studied the emission properties of the sample. Gaussian shaped emission spectra from cryogenic temperatures up to room temperatures were observed, demonstrating the potential of $\mathrm{PbS}$ quantum dots to be used as light emitters in combination with organic matrices. One interesting feature is that the linewidth of the emission spectrum does not follow the expected thermal broadening.
\end{abstract}

\section{INTRODUCTION}

Starting in the 1970s, composites of various inorganic semiconductors, which fulfill certain required technological functionalities, have been developed, further matured, and successively

Distribution Statement A. Approved for public release; distribution unlimited. 
employed in mass-market electronic and optoelectronic devices [1,2]. These heterogeneous materials revolutionized nearly all areas of life. More recently, cost effective chemically tailored hybrids, i.e., the combination of organic and inorganic semiconductors - attributed as a marriage of convenience - attracted growing research interest about ten years ago [3,4]. Boosted by the envisaged technological impact of hybrid nanoscience, the research focused on the chemical bonding essentials of inorganic (metals and semiconductors) quantum dots (QDs) on polymetric surfaces and the embedding in polymer matrices $[5,6]$. The production of polymer-inorganic nanocomposites (PINCs) is equally motivated by economical and technological reasons [7], i.e., the fairly low production costs of polymers, their intrinsic capability of "free-shaping" combined with their low-weight and elasticity are an extremely attractive host for inorganic additives in the form of QDs. Various methods are used to produce PINCs: synthetic techniques, physical methods, electrochemical formation, chemical vapor deposition, and electrophoretic deposition [7-9]. Given that photoluminescence (PL) is the prerequisite for light emitting device structures, the PL properties of various QDs, such as CdS, CdTe [10,11], and PbS [12-14], have been intensively studied. The clearly enhanced quantum confinement with respect to most III-V and II-VI compound semiconductors [15], explains the explicit interest in QDs of the IV-VI semiconductor $\mathrm{PbS}$. In this article, we present our first attempt to form light emitting colloidal $\mathrm{PbS}$ QD films on polyethylene terephthalate (PET).

\section{EXPERIMENTAL DETAILS}

A commercial solution of $4.7 \mathrm{~nm} \mathrm{PbS}$ QDs in toluene with oleic acid as ligands was used to fabricate the sample, i.e., $12.5 \mu \mathrm{L}$ of the PbS QDs in a toluene solution $(40 \mathrm{mg} / \mathrm{mL})$ with oleic acid as capping reagents were added to $5 \mathrm{~mL}$ ethanol in an $8 \mathrm{~mL}$ sample vial with the PET substrate on the bottom. The deposition of the particles from the solution was enforced by using precipitation in a centrifuge deposition method (CDM) for $8 \mathrm{~min}$ at $3200 \mathrm{rpm}$. Afterwards, by removing the solution and drying the sample in air, the QD film was obtained (Figure 60a). Thus, in contrast to previous works, which embedded the QDs in polymers or used PET as a carrier for Fano filters containing PbS QDs [5,6,10,16], we straightforwardly created a QD film on PET hybrid material. A transmission electron microscope (TEM) image of colloidal PbS QDs, deposited under the identical conditions as for the PET substrate but on a copper TEM grid, is shown in Figure 60b. The TEM image reveals the random nature of the QD deposition, but overall shows a fairly continuous film. Because the deposition process is due to centrifugal force, there is no mechanism for the QDs to rearrange into close-packed, uniform layers and leads to partial clustering. Overall, at optical microscope resolutions, the QD film appears uniform with good coverage of the ( $4.5 \mathrm{~mm}$ by $4.5 \mathrm{~mm})$ PET surface.

We do not know the bonding specifics between the QDs and the PET but QDs can bind to polymeric surfaces through van der Waals forces [17], hydrogen-bonding [5], electrostatic linkage [18], and covalent bonding [7]. Furthermore, ligand stabilized QDs are able to bond to specific sites on monomers [19]. To test for weak binding processes, like van der Waals and electrostatic linkage, we attempted to remove the QDs from the PET through several processes. The QD films did not peel off during bending, and light mechanical abrasion. For instance, the $\mathrm{PbS}$ QD film did not come off with a scotch tape test or when rubbed with a cotton tip, indicating that the PbS QD film on PET was robust enough for potential device applications. One advantage of this relatively simple deposition process is that it creates a durable QD film on a flexible substrate in which the QDs are readily available on the PET surface for further

Distribution Statement A. Approved for public release; distribution unlimited. 
functionalization or interaction with external media. In addition, the QD film had measurable light emission at $1348 \mathrm{~nm}$ at room temperature which shows promise for future optical devices. The emission properties of the QD/PET hybrid are our primary interest.

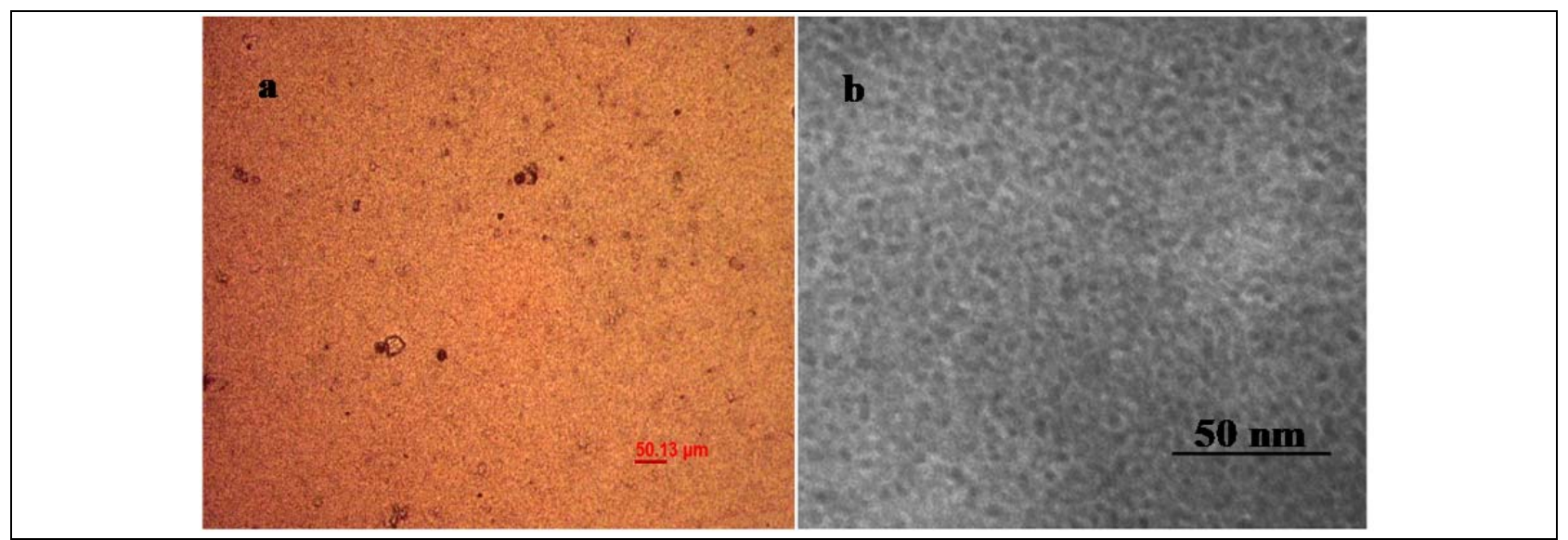

Figure 60. (a) Optical microscope image of PbS QD film on PET (10X magnification), (b) TEM image of PbS QDs on a TEM copper grid, both samples fabricated using CDM.

\section{DISCUSSION}

For the PL experiments, the optical excitation of about $30 \mathrm{~W} / \mathrm{cm}^{2}$ was provided by the $532 \mathrm{~nm}$ continuous wave (cw) emission of a solid-state laser. The PL was measured by a double modulation Fourier transform infrared spectroscopy (FTIR) technique [20]. The double modulation technique was necessary due to the rather weak PL of the sample at room temperature. The luminescence was measured using a nitrogen cooled InGaAs detector and a quartz beamsplitter in the Bomem DA3 FTIR. The luminescence was measured from $5 \mathrm{~K}$ up to $300 \mathrm{~K}$ using a closed-cycle refrigeration cryostat with a diamond window.

Figure 61 shows the PL spectra measured at $5 \mathrm{~K}$ and $300 \mathrm{~K}$. The broken line represents the measured PL intensity $I$ as a function of the emitted photon energy $h v$ and the solid line the fit using the Gaussian intensity distribution,

$$
I(h v)=A \times \exp \left(-\left(h v-h v_{\mathrm{p}}\right)^{2} /\left(2 w^{2}\right)\right)
$$

where $A$ is the peak height, $h v_{\mathrm{p}}$ is the center energy of the PL peak, and $w$ defines the peak width parameter, which is related to the full-width at half maximum (FWHM) by FWHM $=2.35 \times w$. While the PL emission is weaker at $300 \mathrm{~K}$, as expected, the decrease is less than an order of magnitude, which is quite good. The same QD starting solution when dried on a glass substrate showed a much larger peak shift $(100 \mathrm{meV})$ and intensity drop with temperature [21]. The 40 meV PL peak shift from $5 \mathrm{~K}$ to $300 \mathrm{~K}$ in the QD/PET sample is comparable to the $30 \mathrm{meV}$ shift in the QD/glass absorption peak.

There are a few notable differences for the CDM deposited PbS QDs on PET and other QD films formed by a supercritical $\mathrm{CO}_{2}$ fluid process or solution deposition on GaAs and glass, respectively. [13,14] For instance, the temperature dependent shift of the PL peak energy is linear (see Figure 62) versus following the expected Fan equation [14] behavior, especially at

Distribution Statement A. Approved for public release; distribution unlimited. 
low temperatures $(<50 \mathrm{~K})$ where there should be very little change in the emission line energy. This linear behavior is related to alterations of the electronic state of the QDs caused by the laser irradiance [14]. We also noticed that the data are more scattered in Figure 62 than other fits we have reported. Hence, it is possible that colloidal PbS QDs on PET substrates are subject to photo-induced changes under laser irradiation more than PbS QDs on inert substrates such as glass, altering the established bonding linkages at the PET surface build-up during deposition. It seems that the organic substrate plays an active part in the thermal properties of the PL spectra of $\mathrm{PbS}$ QDs. On the other hand, the PL peak intensity vs. temperature in Figure 63 is very similar to the result reported for PbS QDs on glass [22].

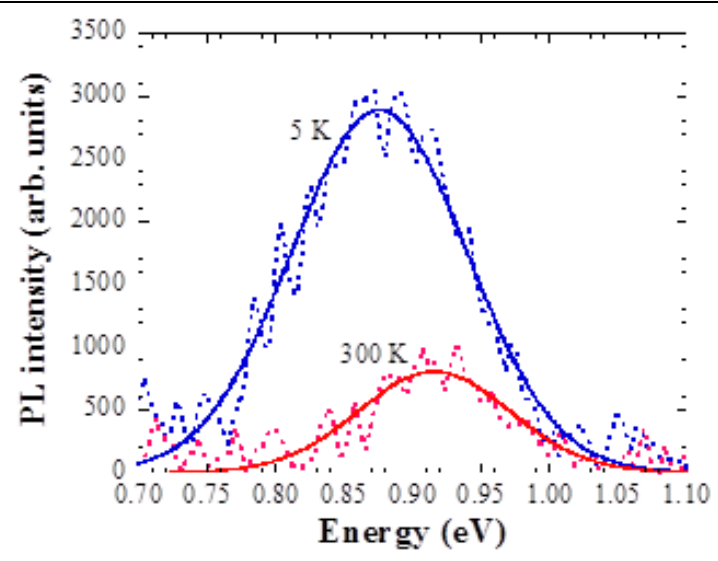

Figure 61. PL spectra at $5 \mathrm{~K}$ and $300 \mathrm{~K}$. The broken line was measured and the solid lines represent the fits using Equation (25).

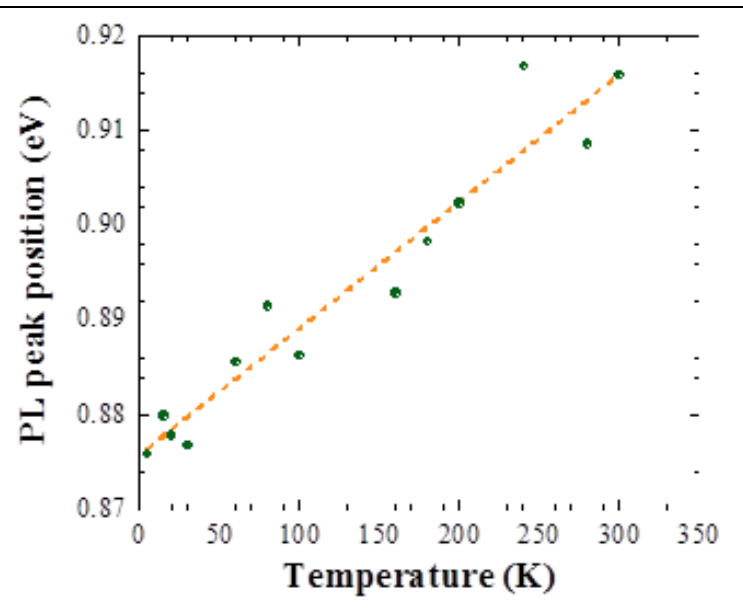

Figure 62. Peak energy position vs. temperature evaluated with the Gaussian fits using Equation 25.

Distribution Statement A. Approved for public release; distribution unlimited. 


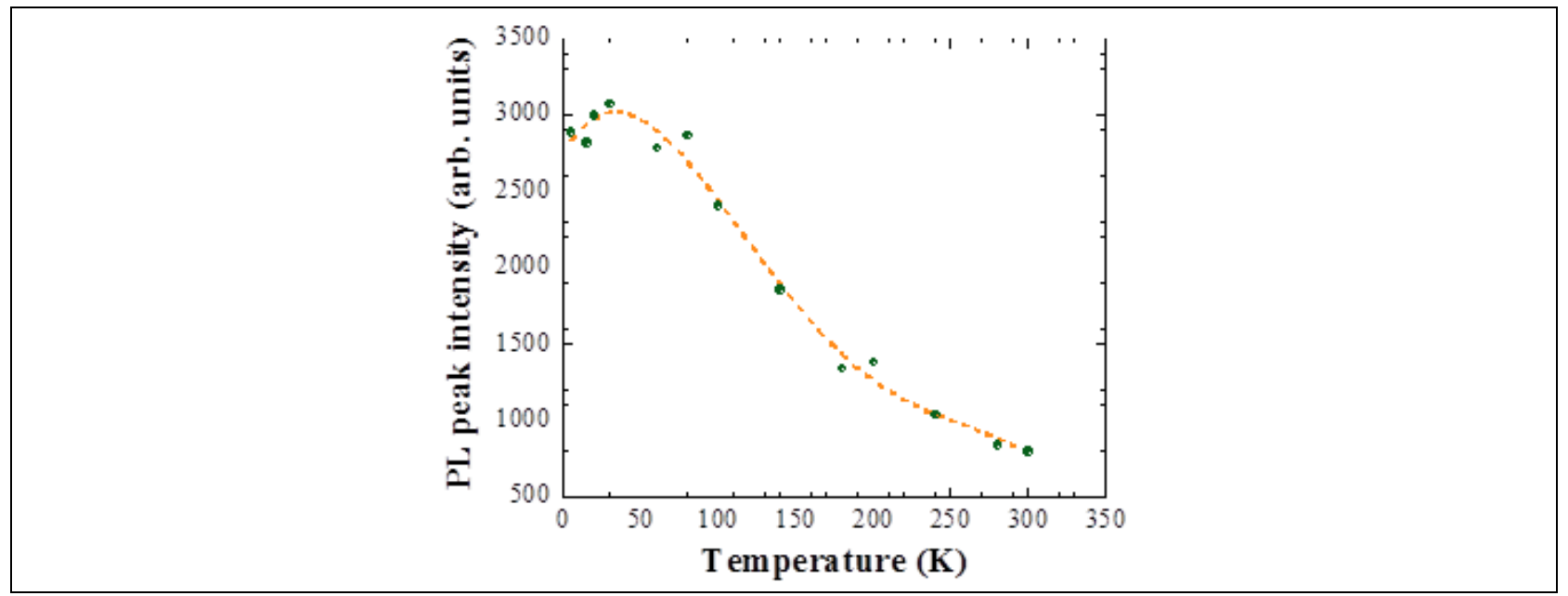

Figure 63. PL peak intensity (parameter $A$ in Equation 25) vs. temperature evaluated from the Gaussian fits.

Another notable difference for the CDM deposited PbS QDs on PET versus other QD films we previously studied is the PL peak FWHM is actually narrower at $300 \mathrm{~K}$ than at $5 \mathrm{~K}$. The result is unusual because it differs considerably from the previously reported thermally induced FWHM broadening, which was described by the Bose distribution function of longitudinal optical (LO) phonons [13,22,23]. The FWHM does broaden from $5 \mathrm{~K}$ to $150 \mathrm{~K}$ (see Figure 64) but then begins to narrow for $\mathrm{T}>200 \mathrm{~K}$. There are very few mechanisms that could potentially explain a narrowing of the PL FWHM and capable of negating thermal broadening. The question is what recombination mechanism dominates at higher temperatures that is not a factor below $200 \mathrm{~K}$ ? One example where the PbS QD environment caused a reduction in the PL FWHM is found in the work of Lin et al. [24], who investigated the PL of PbS QDs in different solutions including methanol, which is chemically related to PET. The quantum yield of PbS QDs in methanol is as low as $9 \%$, while a quantum yield of $82 \%$ is achieved with PbS QDs in toluene, and by lowering the polarity of the hosting solution, the FWHM of the PL shrinks [24]. In our case, other than the duration of laser irradiation, only the sample temperature was changed - so to have a similar change in the QD environment, an alteration in the charge surrounding of the QD is required. This effect might occur if there is a change in the charge trapping at the QD surface above $150 \mathrm{~K}$. However, other possible temperature dependent mechanisms need to be considered. Exploring the cause of the emission line width narrowing is left to further studies.

Distribution Statement A. Approved for public release; distribution unlimited. 


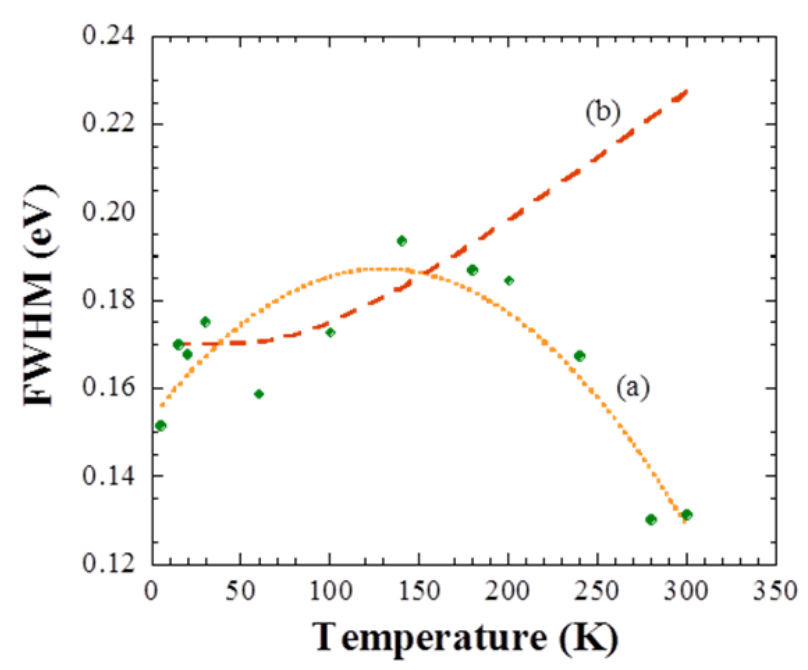

Figure 64. FWHM dependence of the PL spectra vs. temperature: The symbols along curve (a) represent the FWHM determined from the PL fits using Equation (25). The guide for the eyes, the dotted line, is a polynomial fit. The broken line (b) shows the calculated expected behavior based on the Bose distribution function of longitudinal optical phonons $[13,22]$.

\section{CONCLUSIONS}

In conclusion, we have demonstrated that the merger of PbS QDs with an organic substrate results in a light emitting surface structure, demonstrating the possible technological application potential of PINCs at ambient conditions. The centrifuge deposition process used in the sample fabrication is very straightforward and creates a durable QD film on PET substrates. The CDM process is amenable to deposition on a wide variety of substrates. We have used this process to deposit PbS QDs on paper, metal foil, glass and GaAs substrates. The photoluminescence results promote further research on these hybrids in order to optimize the emission features through improvements of the colloid deposition process, and to understand the mechanisms, which cause the uncommon temperature dependence of the PL line width.

\section{REFERENCES}

1. S. O. Kasap, Principles of Electronic Materials and Devices, 2nd Edition (McGraw Hill, New York, 2002).

2. M. Grundmann, The Physics of Semiconductors (Springer, Berlin, 2006).

3. R. Schroeder and B. Ullrich, Appl. Phys. Lett. 81, 556 (2002).

4. E. Gerstner, Nature: Materials Science and Nanotechnology - News \& Features, July 2002.

5. W. H. Binder, C. Kluger, M. Josipovic, C. J. Straif, G. Friedbacher, Macromolecules 39, 8092 (2006).

6. W. H. Binder, M. Lomoschitz, R. Sachsenhofer, G. Friedbacher, J. Nanomaterials, Article ID 613813, (2009).

7. S. Li, M. M. Lin, M. S. Toprak, D. K. Kim, M. Muhammed, Nano Reviews 1, 5214 (2010).

8. S. Guenes, K. P. Fritz, H. Neugebauer, N. S. Sariciftci, S. Kumar, and G. D. Scholes, Solar Energy Materials \& Solar Cells91, 420 (2007).

Distribution Statement A. Approved for public release; distribution unlimited. 
9. J. Akhtar, M. Afzaal, M. A. Vincent, N. A. Burton, I. A. Hillier, and P. O'Brien, Chem. Comm. 47, (2011).

10. H. Du, G. Q. Xu, W. S. Chin, L. Huang, W. Ji, Chem. Mater. 14, 4473 (2002).

11. I. Gorelikovand E. Kumacheva, Chem. Mater. 16, 4122 (2004).

12. W. Lü, F. Yamada, and I. Kamiya, J. Vac. Sci. Technol. B 28, C5E8 (2010).

13. B. Ullrich, X. Y. Xiao, and G. J. Brown, J. Appl. Phys. 108, 013525 (2010).

14. B. Ullrich, J. S. Wang, and G. J. Brown, Appl. Phys. Lett. 99, 081901 (2011).

15. F. W. Wise, Acc. Chem. Res. 33, 773 (2000).

16. L. Chen, H. Yang, Z.Qiang, H. Pang, L. Sun, Z. Ma, R. Pate, A. Stiff-Roberts, S.Gao, J.Xu, G. J. Brown, and W. Zhou, Appl. Phys. Lett. 96, 083111 (2010).

17. Y. Min, M. Akbulut, K. Kristiansen, Y. Golan, J. Israelachvili, Nature Materials 7, 527 (2008).

18. S. Kinge, M. Crego-Calama, D. N. Reinhoudt, ChemPhysChem 9, 20 (2008).

19. A. Haryono, W. H. Binder, Small 2, 600 (2006).

20. B. Ullrich and G. J. Brown, Rev. Sci. Instrum. 83, 016105 (2012).

21. B. Ullrich, J. Wang, X. Xiao, G. Brown, Spectroscopy Photonics International Engineering (SPIE), 8271, 82710A-1 (2012).

22. L. Turyanska, A. Patanè, M. Henini, B. Hennequin, and N. R. Thomas, Appl. Phys. Lett. 90, 101913 (2007).

23. M. S. Gaponenko, A. A. Lutich, N. A. Tolstik, A. A. Onushchenko, A. M. Malyarevich, E. P. Petrov, and K. V. Yumashev, Phys. Rev. B 82, 125320 (2010).

24. W. Lin, K. Fritz, G. Guerin, G. R. Bardajee, S. Hinds, V. Sukhovatkin, E. H. Sargent, G. D. Scholes, and M. A. Winnik, Langmuir 24, 8215 (2008).

\title{
3.6 Stability Studies of Lead Sulfide Colloidal Quantum Dot Films on GaAs
}

Joanna S. Wang*, Elizabeth H. Steenbergen, Howard E. Smith, Lawrence Grazulis, Jeremy Massengale, Bruno Ullrich, and Gail J. Brown, Air Force Research Laboratory, Materials \& Manufacturing Directorate, Wright Patterson AFB, $\mathrm{OH}$ 45433-7707, USA

\begin{abstract}
The stability of colloidal PbS quantum dot (QD) films deposited on various substrates including glass and GaAs was studied. Over a period of months, the QD film sample was re-tested after being left unprotected in air under ambient conditions. Despite exposure to $532 \mathrm{~nm}$ laser excitation and cooling to cryogenic temperatures, the initial photoluminescence (PL) remained stable between tests. To track potential changes to the QDs over time, X-ray photoelectron spectroscopy (XPS), transmission electron microscopy (TEM), X-ray diffraction (XRD), optical microscopy, UV-Vis-NIR spectrophotometry and atomic force microscopy (AFM), were employed. Evidence points towards oxidation enforced shrinking of the active QD volume causing a blue shift of the absorption and photoluminescence. The presented studies are important for reliability expectations of light emitters based on PbS QDs.
\end{abstract}

Distribution Statement A. Approved for public release; distribution unlimited. 
Keywords: $\mathrm{PbS}$ quantum dots, photoluminescence, deposition, supercritical $\mathrm{CO}_{2}, \mathrm{UV}-\mathrm{Vis}-\mathrm{NIR}$ spectrophotometry, X-ray photoelectron spectroscopy, X-ray diffraction, atomic force microscope.

\section{INTRODUCTION}

Thin films of semiconductor quantum dots (QDs) are emerging as an important class of materials for electronic and optoelectronic devices such as field-effect transistors [1-3], photodetectors [47], light-emitting diodes [8-10], metamaterials [11-13], and solar cells [14,15]. As a consequence, the optical and electrical stability of colloidal quantum dots, such as $\mathrm{PbS}$, used in devices is an important issue. For example, the effects of photo-oxidation exposure on the surface state of colloidal $\mathrm{PbS}$ QD films have drawn attention during the last decade [16-20]. Detrimental impacts to the morphology and optical properties of PbS QD films can occur via processes such as oxidation, heating or UV induced degradation. The oxidation-induced reduction in the size of the $\mathrm{PbS}$ "core" increases quantum confinement, causing shifts of the PL peak and the absorption onset to higher energies. To date, most investigations of IV-VI QDs' stability have focused on thermally activated oxidation of $\mathrm{PbX}$ QD solutions or solid films, which rapidly decreased the physical and electronic size of the QDs, increasing the confined state energy separation and causing excitonic blueshifts of absorption and emission spectra [1618]. However, the rate of aging of PbS QDs in ambient environments has not been thoroughly studied.

In this study, the stability of the PbS QD films, under ambient conditions was tested, with particular attention to any changes in the oxidation state of the QDs over a period of several months. Two sets of PbS QD films were fabricated, one set on glass and the other on $1 \mathrm{~cm}$ by 1 $\mathrm{cm}$ pieces of semi-insulating GaAs. The impact of the PbS QD film deposition processes on QD stability was also studied in these two sets. The aging of these films was investigated using photoluminescence (PL), UV-Vis-NIR spectrophotometry, X-ray photoelectron spectroscopy (XPS), transmission electron microscopy (TEM), powder X-ray diffraction (XRD), and atomic force microscopy (AFM). Fundamental investigations are required to systematically measure the rate of any degradation in the films due to short term exposure to ambient conditions during material and device fabrication processes, and to explore the need for device encapsulation over the long term. As reported in the literature [18], QD oxidation causes a considerable blue shift of both the absorption and the luminescent transitions. Therefore, the optical properties are an accurate means for tracking changes in the QD oxidation.

\section{SAMPLE PREPARATION AND OPTICAL MEASUREMENTS}

The films were formed employing supercritical fluid $\mathrm{CO}_{2}$ deposition (SFD and solvent deposition (SDM) methods. Oleic acid-capped $\mathrm{PbS}$ nanoparticles were synthesized in our lab using a procedure reported by Hines and Scholes [21]. The synthesized PbS QDs were dispersed in toluene. The SFD process was carried out using a $35.3 \mathrm{~mL}$ high-pressure stainless steel chamber. For both the glass slide and GaAs substrates the QD solution was contained on top of the substrate for the solvent removal by the SFD process. For the glass substrates a special apparatus was designed to cover larger diameters uniformly but the GaAs was too fragile for this apparatus so smaller $(1 \mathrm{~cm}$ by $1 \mathrm{~cm})$ squares of GaAs were placed in a small vial with the solution and then placed in the high pressure chamber. Presumably, the supercritical fluid $\mathrm{CO}_{2}$ deposition is governed by a gas-antisolvent (GAS) mechanism described previously in the

Distribution Statement A. Approved for public release; distribution unlimited. 
literature [22-24], where an increasing amount of $\mathrm{CO}_{2}$ alters the polarity of the toluene solvent and becomes unfavorable for particle stabilization in the colloid, thus resulting in the particles precipitating from solution. The PbS QDs precipitate evenly and self-assemble in a uniform array on the substrates during SFD. The method was reported previously for making gold nanoparticle arrays on Si surfaces [22-24]. The oleic acid protected PbS QDs can also form films on the substrates immersed in the toluene solution under atmospheric pressure. This procedure is referred to as SDM. However, the benchtop solvent evaporation process, due to high surface tension at the liquid/vapor interface, can lead to imperfect nanoparticle ordering, forming isolated islands, percolating domains, locally high particle populations, and uneven surface coverage [25-27].

The optical images of PbS QDs on GaAs and glass fabricated by the SFD and SDM methods were taken by using Axiotron II Inspection Microscope. The solution deposition method using $p$ GaAs substrates resulted in coffee rings, long range channels and fractures in both 10 and 50 fold magnifications. However, SFD samples revealed a uniform material coverage over the entire substrate area, with some fractures. These fractures probably are due to the dryness via solvent removal in the sample deposition process. The initial optical images were taken immediately after the deposition process. For example, Figure 65 shows the initial images of SDM and SFD films on semi-insulating (S.I.) GaAs. Despite the colors seen in the images below, the PbS QD films appear gray to the naked eye. Based upon the images taken at both 10 and 50 fold magnifications, the microscope images from initial and final measurements months later closely resemble each other. This gives an indication that the structural stability of the PbS QD films is quite reliable.

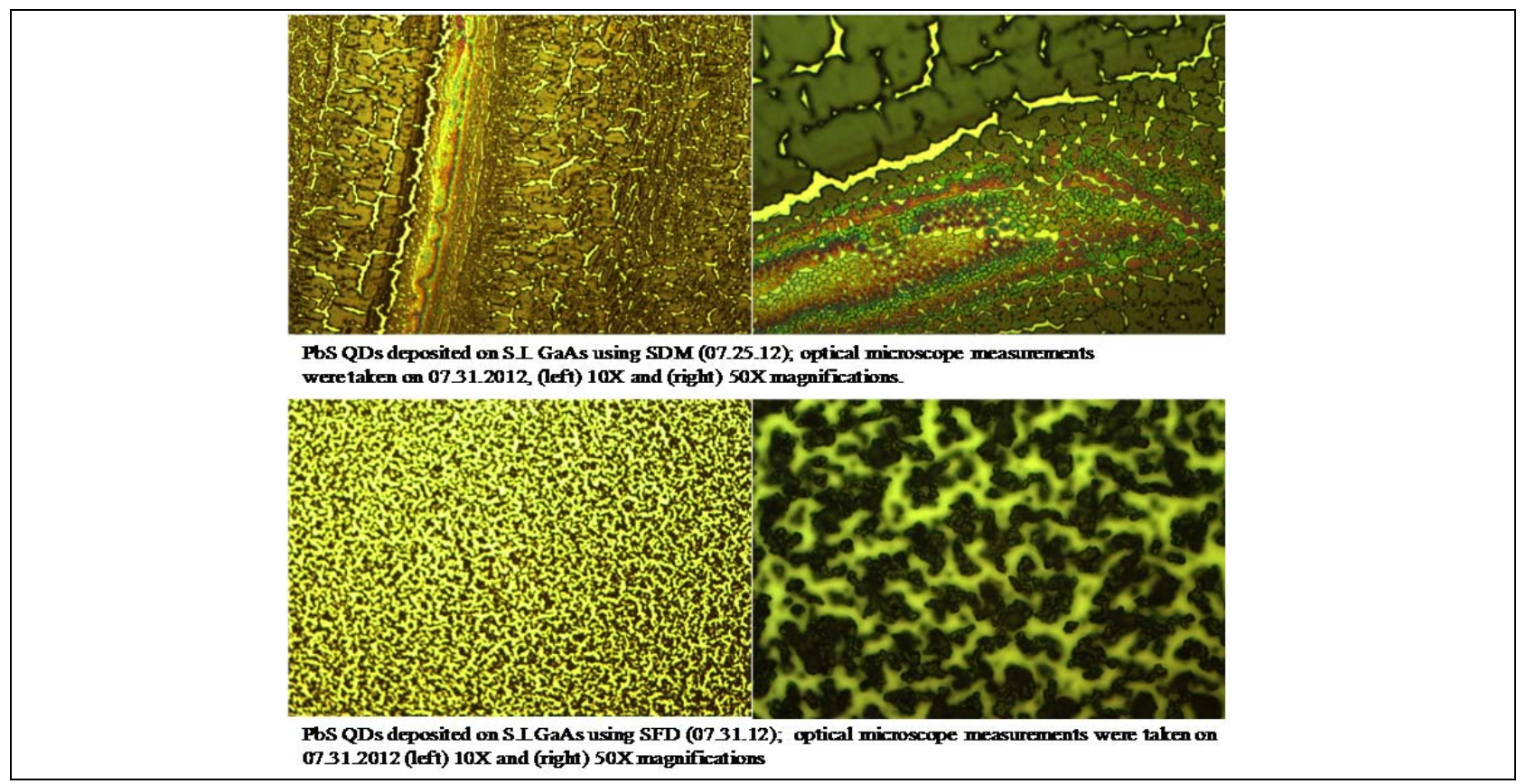

Figure 65. Optical microscope images of (top) PbS (SDM) on S.I. GaAs substrate and (bottom) PbS (SFD) on S.I.GaAs substrate.

Distribution Statement A. Approved for public release; distribution unlimited. 


\section{RESULTS AND DISCUSSION}

\section{Absorption studies of PbS films deposited by SDM and SFD}

The UV-Vis-NIR spectra of PbS QDs were obtained using a Cary 5000 Varian UV-Vis-NIR spectrophotometer scanning from 400 to $1600 \mathrm{~nm}$. In this study the absorbance of SFD PbS QDs on a glass substrate was measured. The sample remained unprotected under the ambient conditions for 4.3 months, and the absorbance was measured again. About a $20 \mathrm{~nm}$ blue-shift was observed from the initial $1304 \mathrm{~nm}$ peak wavelength to the final $1284 \mathrm{~nm}$ peak wavelength. The peak shift is presumably caused by an oxidizing effect due to the exposure to air. The oxidation of $\mathrm{Pb}$ and $\mathrm{S}$ on the outer surface of the QDs reduces the active size and thereby creates a blue-shift [18], as shown in Figure 66 (top). In the same manner the PbS QD film deposited on glass by SDM was measured. The fabricated sample was stored at ambient conditions and was measured again $\sim 4.6$ months later. During this period of time the maximum peak wavelength blue-shifted $34 \mathrm{~nm}$ from $1304 \mathrm{~nm}$ initially to $1270 \mathrm{~nm}$ as shown in Figure 66 (bottom). The absorbance of the films made by the SFD method shifted less than SDM films because the SDM deposited films possess a relative larger surface area due to the uneven, porous, and "coffee ring" changes in density, which presents favorable conditions for oxidation. On the other hand, the films deposited by SFD possess a compact and uniform coverage, which is less influenced by air.

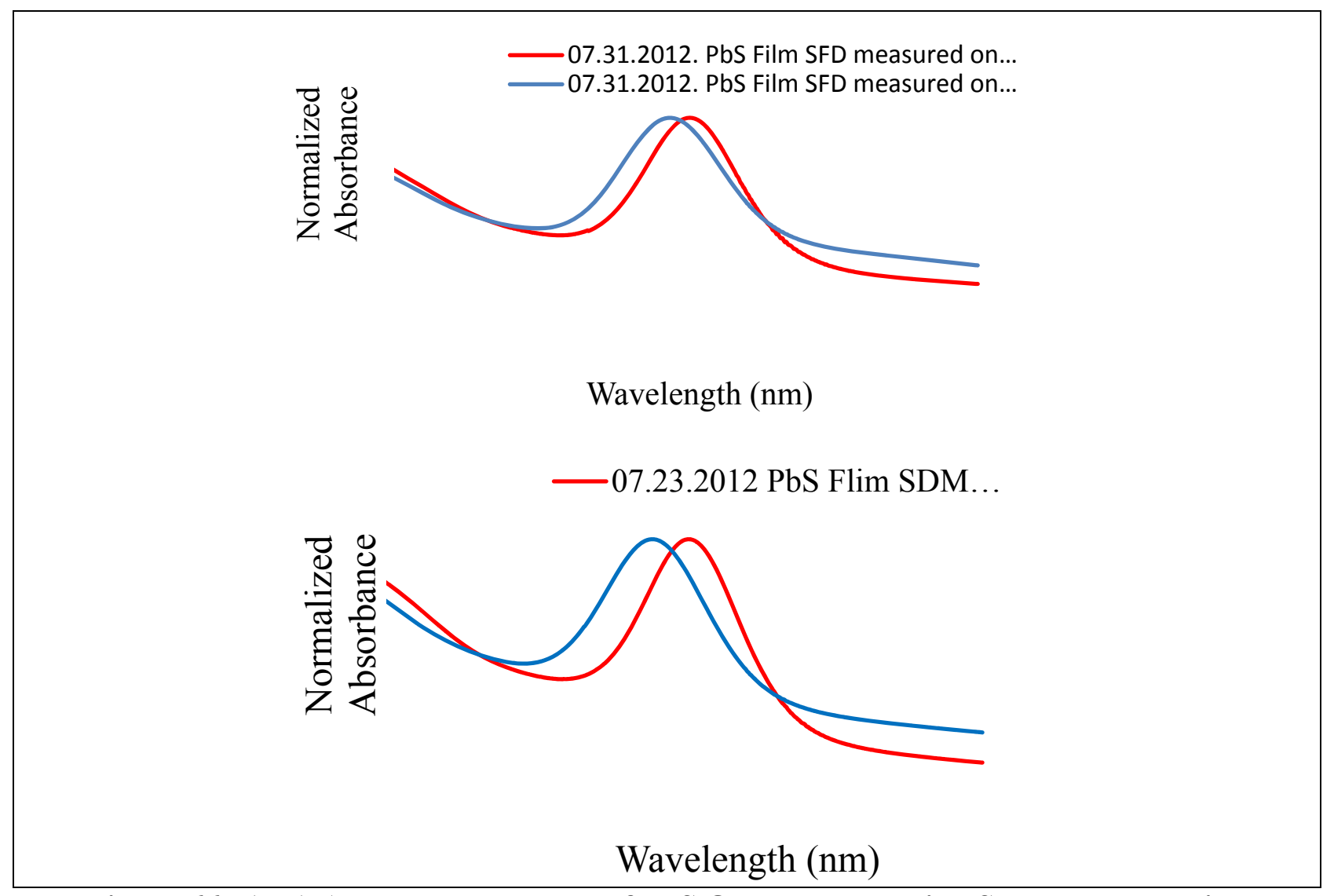

Figure 66. (top) Absorbance spectra of PbS QDs on glass using SFD. The duration between 2 measurements is 4.3 months; (bottom) Absorbance spectra of PbS QDs on glass using SDM. The duration between 2 measurement is around 4.6 months.

Distribution Statement A. Approved for public release; distribution unlimited. 


\section{Photoluminescence study of PbS QD films over three months}

Room-temperature photoluminescence (PL) of the four samples was measured approximately once a month for three consecutive months, with the first of these measurements made approximately two months after QD deposition and storage in air. The samples were measured in a cryostat with a CsI window and optically pumped with a frequency-doubled Nd:YAG laser $(532 \mathrm{~nm})$ at an intensity of $0.76 \mathrm{~W} / \mathrm{cm}^{2}$. A Bomem DA3 FTIR spectrometer was used to collect the PL spectra. Each spectrum was fit with a Gaussian curve to determine the peak position and FWHM, and the data are shown in Table 9.

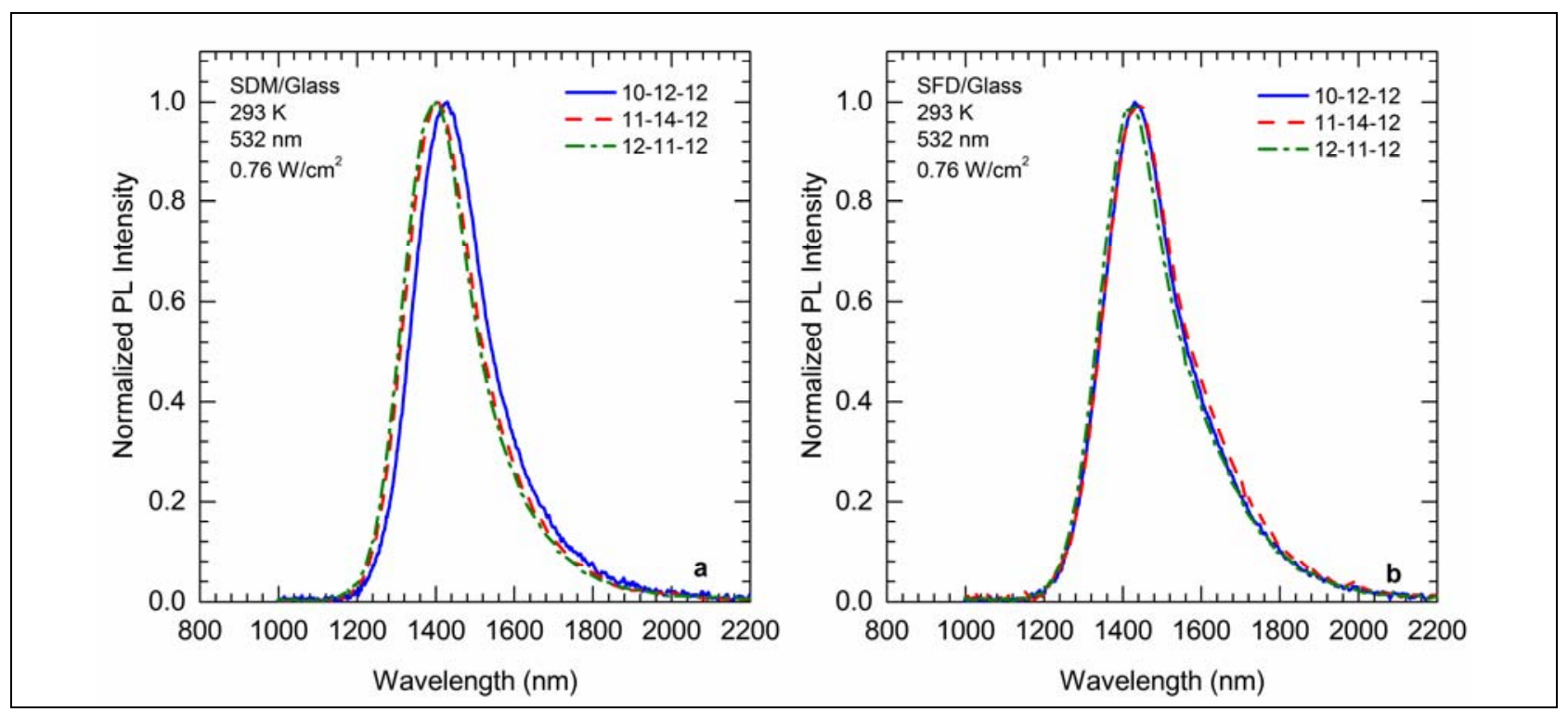

Figure 67. Room temperature photoluminescence measurements over three months on samples a) SDM and b) SFD deposited on glass.

Figure 67 displays the normalized PL spectra for the SDM (Figure 67a) and SFD (Figure 67b) samples on glass. From the first month to the second month, a blue shift in the PL peak position of $21 \mathrm{~nm}(13 \mathrm{meV})$ for the SDM sample was observed while the FWHM increased by $2 \mathrm{meV}$. The second to the third month shows no change in the FWHM and a smaller change in the peak blue shift, only $8 \mathrm{~nm}(5 \mathrm{meV})$. The SFD sample on glass first experiences a small red shift in the PL peak position, $5 \mathrm{~nm}(3 \mathrm{meV})$, from the first to the second month and then a subsequent $13 \mathrm{~nm}$ $(8 \mathrm{meV})$ blue shift from the second to the third month. The FWHM varied by $4 \mathrm{meV}$ over the three months. These shifts in the peak position for the SFD sample are within the experimental error of $\pm 5 \mathrm{meV}$, so effectively this sample showed no change in the PL spectra over the three months.

The normalized spectra for the samples deposited on GaAs are shown in Figure 68: SDM (Figure $68 \mathrm{a})$ and SFD (Figure 68b). The PL peak position for the SDM sample blue shifts by $16 \mathrm{~nm}$ (10 $\mathrm{meV})$ and $11 \mathrm{~nm}(7 \mathrm{meV})$ from the first to the second and the second to the third months, respectively. The FWHM is the smallest for this sample and varies by $5 \mathrm{meV}$ over the three months. The SFD sample peak position and FWHM remain essentially constant from the first to the second month, varying only by $3 \mathrm{~nm}(2 \mathrm{meV})$, but a substantial red shift of $48 \mathrm{~nm}(27 \mathrm{meV})$ is observed in the third month along with a large decrease in the intensity resulting in more noise on the spectrum. The red shift and intensity decrease are drastic and unexpected; however, this

Distribution Statement A. Approved for public release; distribution unlimited. 
was the only sample that was thermally cycled to $4 \mathrm{~K}$ for temperature-dependent PL measurements.

The SFD samples experienced less change in the PL peak position over the first two months compared to the SDM samples, $5 \mathrm{~nm}(3 \mathrm{meV})$ and $3 \mathrm{~nm}(2 \mathrm{meV})$ versus $21 \mathrm{~nm}(13 \mathrm{meV})$ and 16 $\mathrm{nm}(10 \mathrm{meV})$, respectively. From the second to the third month, the peak position of the SDM samples shifted by half the amount from the first to the second month, while the peak shift of the SFD samples increased. The change in the FWHM of all the samples was less than 5 meV over the three months. Overall, the PL of the SFD samples was more stable than that of the SDM samples.
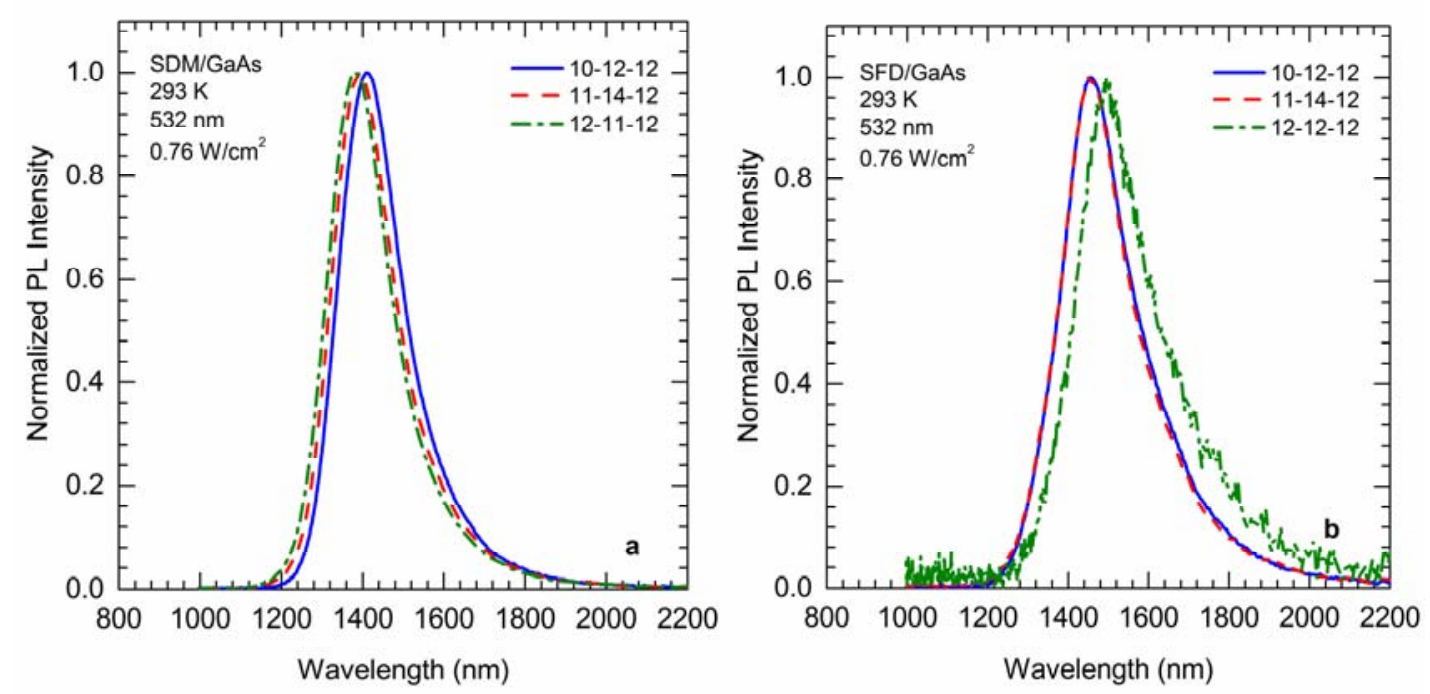

Figure 68. Room temperature photoluminescence measurements over three months on samples a) SDM and b) SFD deposited on GaAs.

Distribution Statement A. Approved for public release; distribution unlimited. 
Table 9. Summary of Gaussian fit parameters to the PL spectra.

\begin{tabular}{|c|c|c|c|c|}
\hline Method & Substrate & Sample & $\begin{array}{c}\text { Peak } \\
\text { Position } \\
(\mathrm{nm}, \mathrm{meV})\end{array}$ & $\begin{array}{c}\text { FWHM } \\
(\mathrm{meV})\end{array}$ \\
\hline \multirow[t]{4}{*}{ SDM } & Glass & $7 / 23 / 2012$ & & \\
\hline & $10-12-12$ & & 1432,866 & 141 \\
\hline & 11-14-12 & & 1411,879 & 143 \\
\hline & $12-11-12$ & & 1403,884 & 143 \\
\hline \multirow[t]{4}{*}{ SFD } & Glass & $7 / 31 / 2012$ & & \\
\hline & $10-12-12$ & & 1445,858 & 152 \\
\hline & $11-14-12$ & & 1450,855 & 156 \\
\hline & $12-11-12$ & & 1437,863 & 153 \\
\hline \multirow[t]{4}{*}{ SDM } & GaAs & $7 / 25 / 2012$ & & \\
\hline & $10-12-12$ & & 1417,875 & 123 \\
\hline & $11-14-12$ & & 1401,885 & 126 \\
\hline & $12-11-12$ & & 1390,892 & 128 \\
\hline \multirow[t]{4}{*}{ SFD } & GaAs & $7 / 31 / 2012$ & & \\
\hline & $10-12-12$ & & 1469,844 & 143 \\
\hline & $11-14-12$ & & 1466,846 & 141 \\
\hline & $12-12-12$ & & 1514,819 & 146 \\
\hline
\end{tabular}

\section{X-ray photoelectron spectroscopy (XPS)}

XPS was used to assess the chemical stability of PbS QDs deposited on glass substrates by both the SFD and SDM methods. The $\mathrm{Pb} 2 \mathrm{f}, \mathrm{S} 2 \mathrm{~s}$ and $\mathrm{O} 1 \mathrm{~s}$ transitions were examined for evidence of chemical shift $[28,29]$. The spectra were acquired on a Perkin-Elmer Phi 5100 system, equipped with a non-monochromatic magnesium X-ray source and a hemispherical energy analyzer. Data manipulation and quantification were performed using CasaXPS software [30], applying published atomic sensitivity factors [31]. Chemical state determination was performed by fitting the spectra with component peaks of mixed Gaussian/ Lorentzian (G/L) character. The proper mixture was determined for each spectrum individually, by determining the best statistical fit to its $\mathrm{C} 1 \mathrm{~s}$ peak. The mixture value varied from G55/L45 to G30/L70, presumably due to differences in emission-induced surface charge. The fitting was done after subtracting the photoelectron signal contribution due to excitation by $\mathrm{Mg}$ X-ray source satellites, and after performing Shirley (P 4f, O 1s) or linear (S 2s) background subtraction.

Figure 69 shows the XPS measurements obtained approximately 2 months and 6 months after initial deposition (top and bottom halves of the figure, respectively) from both the SFD and SDM depositions (left and right halves, respectively). Each half of the figure is divided into two pairs of $\mathrm{Pb} 4 \mathrm{f}$ and $\mathrm{S} 2 \mathrm{~s}$ photoelectron transitions. The raw XPS data are represented with red lines. Each of these transitions is broken into its chemical components of $\mathrm{PbS}$ and $\mathrm{PbSO}_{\mathrm{x}}$. Note that each $\mathrm{Pb} 4 \mathrm{f}$ photoelectron has two major peaks corresponding to 5/2 and 7/2 spin-orbit coupling. The time elapsed since deposition is shown inset in each graph in days (d). At approximately two months, for both depositions, the $\mathrm{S} 2 \mathrm{~s}$ and $\mathrm{Pb} 4 \mathrm{f}$ spectra show substantial amounts of two different oxidation states (curves a, c, e and g). The chemical shift is considerably larger for the $\mathrm{S}$

Distribution Statement A. Approved for public release; distribution unlimited. 
$2 \mathrm{~s}$ transition than for the $\mathrm{Pb} 4 \mathrm{f}$ transition, but in both cases the observed shift is consistent with the formation of $\mathrm{PbSO}_{3}$ and $\mathrm{PbSO}_{4}$ [31,32]. That said, the $\mathrm{Pb} 4 \mathrm{f}$ chemical shifts of the various $\mathrm{Pb}_{\mathrm{x}} \mathrm{O}_{\mathrm{y}}$ compounds are difficult to distinguish from $\mathrm{PbS}$ and $\mathrm{PbSO}_{4}$ compounds [28, 31$]$. However, the $\mathrm{O} 1 \mathrm{~s}$ transition (not shown) is able to distinguish the presence of $\mathrm{Pb}_{\mathrm{x}} \mathrm{O}_{\mathrm{y}}$ compounds, and it does not show evidence of $\mathrm{Pb}_{\mathrm{x}} \mathrm{O}_{\mathrm{y}}$. Therefore the two main chemical components observed in both the $\mathrm{Pb} 4 \mathrm{f}$ and $\mathrm{S} 2 \mathrm{~s}$ spectra correspond to $\mathrm{PbS}$ and $\mathrm{PbSO}_{\mathrm{x}}$.

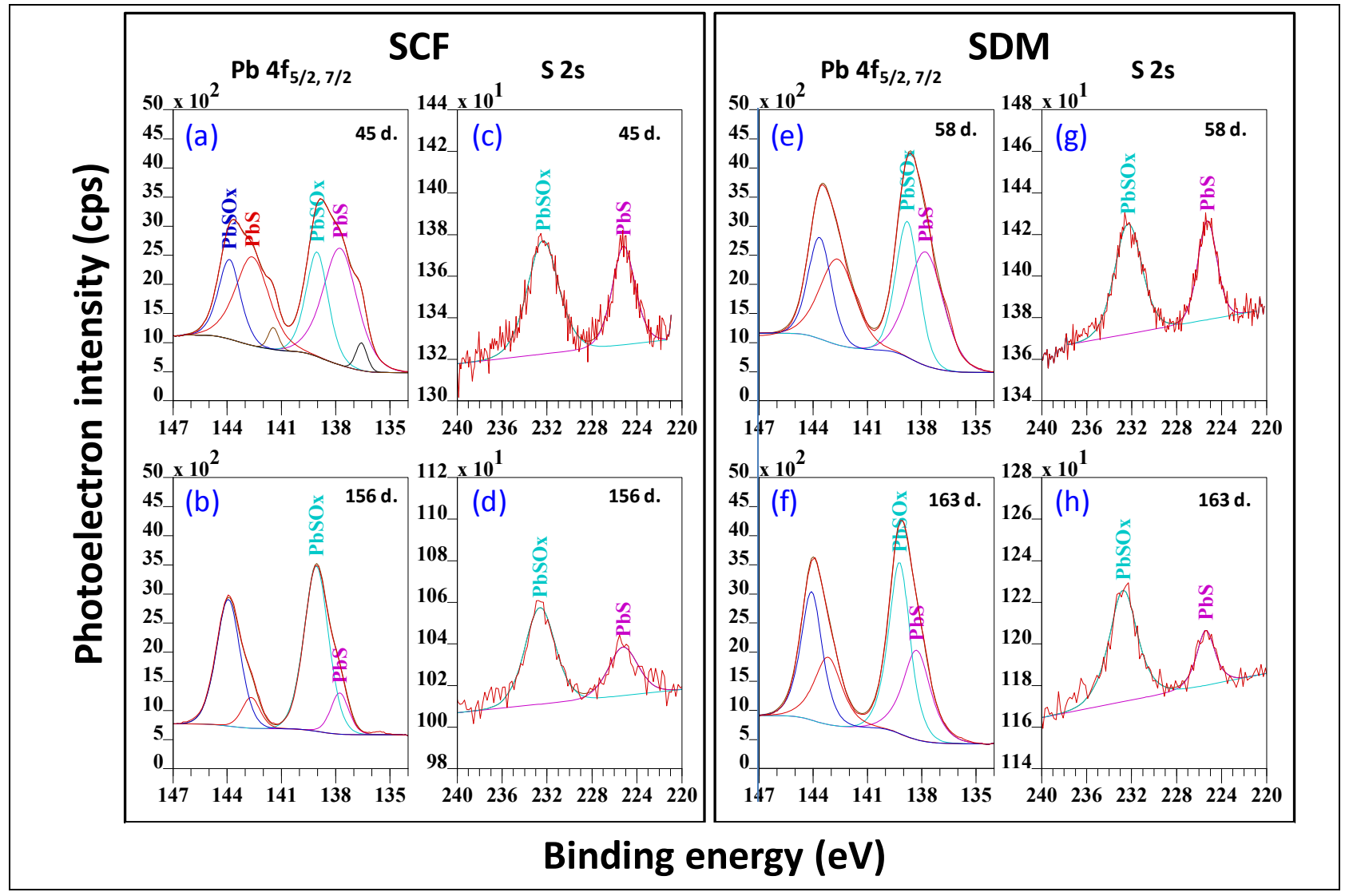

Figure 69. $\mathrm{Pb} 4 \mathrm{f}$ and $\mathrm{S} 2 \mathrm{~s}$ photoelectron transitions obtained from the SFD and SDM of PbS QD films; with chemical states fitted.

In the spectra acquired $\sim 6$ months after deposition (bottom half of the figure), all samples have undergone substantial additional oxidation: the proportion attributed to $\mathrm{PbSO}_{\mathrm{x}}$ has grown and the proportion of $\mathrm{PbS}$ has diminished. The changes are qualitatively similar in both the $\mathrm{S} 2 \mathrm{~s}$ and $\mathrm{Pb}$ $4 \mathrm{f}$ spectra. The S 2s spectra reveal that the oxidation is occurring at the sulfur atom site. And while lead spectra show additional oxidation that could be consistent with the formation of either $\mathrm{PbSO}_{\mathrm{x}}$ or $\mathrm{Pb}_{\mathrm{x}} \mathrm{O}_{\mathrm{y}}$, the $\mathrm{O}$ 1s spectra again establish no evidence for $\mathrm{Pb}_{\mathrm{x}} \mathrm{O}_{\mathrm{y}}$ formation.

\section{X-ray diffraction (XRD) studies of PbS films deposited by SDM and SFD}

The X-ray diffraction (XRD) is a rapid analytical technique primarily used for phase identification of a crystalline material and can provide information on unit lattice dimensions. Powder X-ray diffraction (XRD) patterns were recorded on a Bruker D\&Advance X-ray powder diffractometer with graphite monochromatized $\mathrm{Cu} \mathrm{K} \alpha(\lambda=0.15406 \mathrm{~nm})$ source. A scanning rate

Distribution Statement A. Approved for public release; distribution unlimited. 
of $0.05^{\circ} \mathrm{s}^{-1}$ was applied to record the pattern in the $2 \theta$ range of 10-90 ${ }^{\circ}$. The crystal structure of $\mathrm{PbS}$ is the galena structure and its XRD peak positions are well established.

According to our XRD measurement, the $4.7 \mathrm{~nm}$ sized PbS QDs possess the galena structure as expected. Before we discuss the deposited films stability, it is necessary to understand the nature of the QD aging process and the stability of $\mathrm{PbS}$ QDs in solution. We monitored the degradation of the PbS QDs (4.7 nm) stored in solution over 3 years. During this period the PbS solution was stored at $4{ }^{\circ} \mathrm{C}$ in the dark. The XRD diffraction peaks remained unchanged during the first year. After the $2^{\text {nd }}$ year the PbS QDs in solution gradually decayed and the diffraction peaks slowly diminished except for the 220 peak which was enlarged significantly comparing with the initial measurement. This indicates that the PbS QD solution shelf life after synthesis is about one year. More important, however, is the long term stability of the prepared films.

The initial XRD measurement of a PbS QD film $(4.7 \mathrm{~nm})$ deposited by the SDM method on glass in 2009 is shown in Figure 70 (top). This sample was then left under ambient conditions for an extended period of time. After two and a half years the PbS film was remeasured by XRD (Figure 70 bottom). Overall there is not much phase transformation suggesting the PbS QD film is quite stable when exposed to air under the normal ambient conditions.

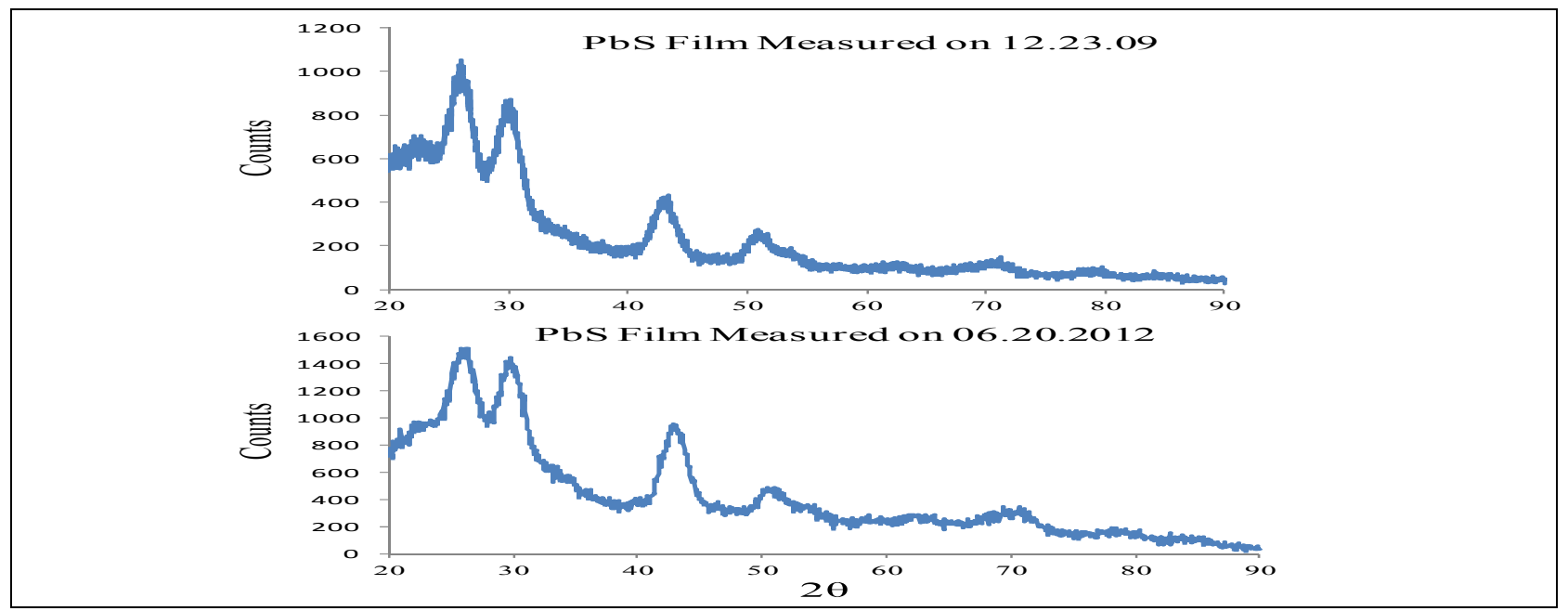

Figure 70. XRD measurements of a PbS film $(4.7 \mathrm{~nm})$ measured in 2009 (top) and 2012 (bottom) which were prepared by SDM.

\section{Atomic force microscope (AFM) studies of PbS films deposited by SDM and SFD}

AFM was used to characterize the general morphology of the PbS QD films deposited by SFD and SDM on both glass and GaAs. Both preparation methods produced similar morphologies when viewed at the micrometer size scale (see Figure 71a, which shows $10 \mu \mathrm{m}$ x $10 \mu \mathrm{m}$ AFM image). Clearly, two types of features are generally observed, i.e. "plateaus" of densely packed QDs and deep cracks. The "plateaus" are $100 \mathrm{~nm}$ to $300 \mathrm{~nm}$ thick relative to the substrate and the cracks are $1 \mu \mathrm{m}$ or less wide. Since the films are not continuous as deposited by either method, a second deposition would be required to fill in these small cracks in order to use the films in electrical devices. The SFD method is well suited for depositing nanoparticles into $<$ $1 \mu \mathrm{m}$ dimensions [22]. Figure $71 \mathrm{~b}$ is a $3 \mathrm{D}$ representation of the same data shown in Figure 71a.

Distribution Statement A. Approved for public release; distribution unlimited. 
Although the fissures might appear to extend to the substrate, higher magnification images from these areas revealed that this was not the case. Instead, QDs are always distributed along the bottom surfaces of these fissures, with an average thickness of 20 to $50 \mathrm{~nm}$, as determined by measuring the height of the QDs relative to scratches made through the QD layer to the substrate. That is, the minimum value of the height scale of Figure $71 \mathrm{~b}$ represents the top of the QD layers within the fissures, some 20 to $50 \mathrm{~nm}$ above the substrate surface.

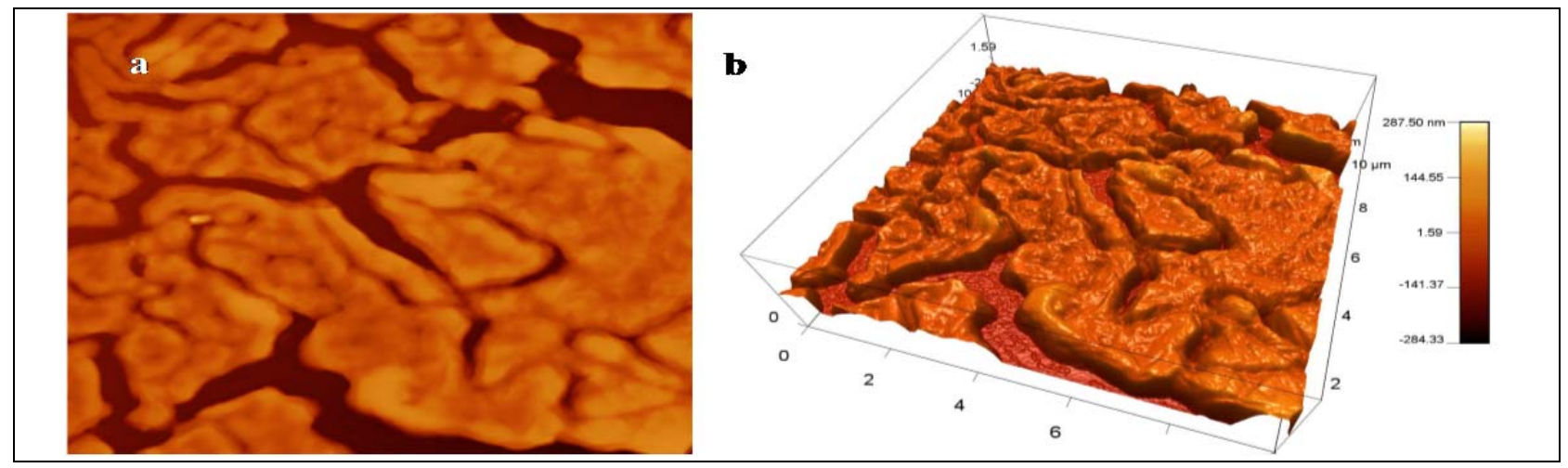

Figure 71. (a) $10 \mu \mathrm{m} \times 10 \mu \mathrm{m}$ image of the general surface morphology; (b) 3D representation of Figure 71a

Transmission electron microscope (TEM) studies of PbS films deposited by SDM and SFD For this part of the aging study we monitored PbS colloidal QDs deposited on carbon coated copper grids over approximately 2 years using SDM and SFD methods. Figure 72 shows the TEM profile of these PbS QDs formed by SDM and SFD. Here, TEM measurements taken on 04/13/10 (initial), on 11/03/11 (1.5 years), and 07/02/12 (2.2 years) are compared. We observed clearly that the QDs aged in air lose their faceting and are more diffuse in shape and size. The active average QD diameter appears to reduce as described in the literature [18]. The shapes of QDs become more irregular, and the size distribution considerably broadens when samples were stored under the ambient conditions. From observation of the TEM images, the QDs both oxidize and ripen in air and ripening occurs mostly by atomic diffusion between immobile QDs rather than by QD diffusion and aggregation. The edges of the QDs in Figures 72c and 72f became more faded than TEM images of Figures 72b and 72e, and those of Figures 72a and 72d.

Distribution Statement A. Approved for public release; distribution unlimited. 


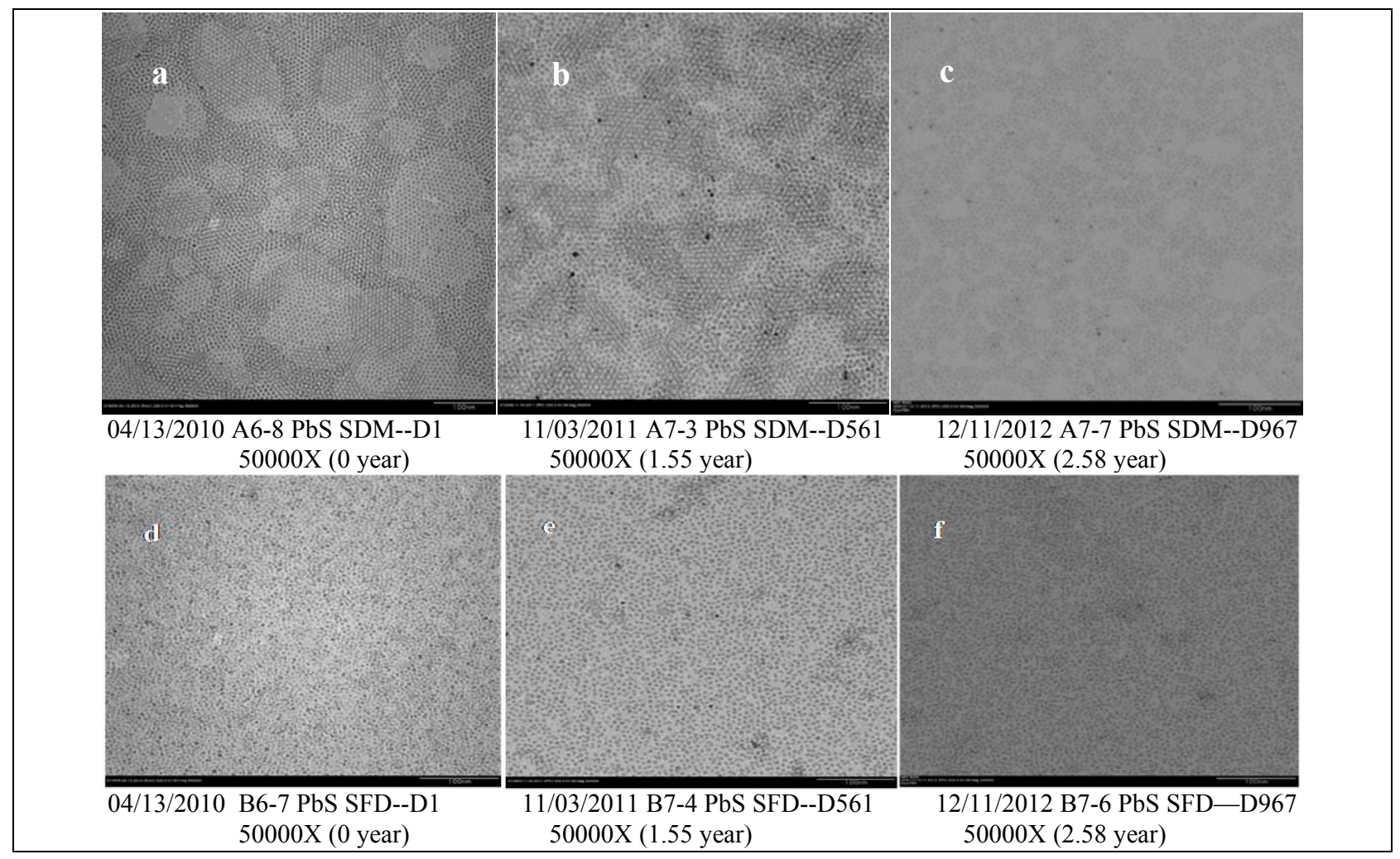

Figure 72. SDM $(a, b, c)$ and $\mathrm{scCO}_{2}(\mathrm{~d}, \mathrm{e}, \mathrm{f})$ deposition of $\mathrm{PbS}$ on carbon coated cupper grids, which were stored under ambient conditions about 2 years and 3 months. TEM images (a,d) were taken on Day 1, TEM images (b,e) were taken on Day 56, and TEM images (c, f) were taken on Day 967.

\section{CONCLUSIONS}

The study presented here provides a detailed overview about the temporal impact of the ambient environment on the optical properties of PbS QDs, such as near infrared absorption and photoluminescence. The stability of the optical properties is of particular importance for optical device fabrication procedures and reliability. We observed changes in the optical properties over the time period of more than 4 months, for PbS QD films deposited from solution by both conventional solvent evaporation and solvent removal by a supercritical fluid $\mathrm{CO}_{2}$ method. The $\mathrm{PbS}$ QD films on GaAs and glass both had good adherence, despite the lack of a polymer matrix, and with the supercritical fluid $\mathrm{CO}_{2}$ deposition process the films have a very uniform substrate coverage without the typical "coffee ring" pattern of standard solution deposition processes.

The optical data point towards slow oxidation of the QD surface despite the protecting oleic acid ligands. The lead oxide and sulfur oxide formation shrinks the active QD volumes, causing a blue shift of the emission and absorption. The absorption process is the more reliable method for tracking changes in the optical volume since it involves the transition between the two quantum confined states. Unfortunately, the emission process involves a transition between trap states and only one of the quantum confined states. The existence of traps in the investigated QDs is supported by the $\sim 100 \mathrm{meV}$ "Stokes" shift [33] between the initial absorption peak (951 $\mathrm{meV})$ and the initial PL peak $(858 \mathrm{meV})$. The oxide formation was confirmed using $\mathrm{x}$-ray photoelectron spectroscopy.

Distribution Statement A. Approved for public release; distribution unlimited. 
The stability of the structural and optical properties of the unprotected QD films in ambient conditions is good over a time period of several months. The denser QD films formed by the SFD are more oxidation resistant than the films formed by the SDM method. Over a period of 4 months the QD optical absorption only shifted by $15 \mathrm{meV}$ for the SFD processed films. This small shift reflects that only a small change in PbS QD volume occurred. For device fabrication the PbS QD layers would not remain unencapsulated and therefore would be even less likely to degrade over longer periods of time. A second PbS QD deposition to fill the micrometer or less cracks should take care of potential electrical continuity issues and testing of degradation of electrical properties needs to be done since these properties may degrade faster than the optical properties [18].

\section{REFERENCES}

[1] Porter, V. J., Mentzel, T., Charpentier, S., Kastner, M. A. and Bawendi, M. G., "Temperature-, gate-, and photoinduced conductance of close-packed CdTe nanocrystal films," Phys. Rev. B, 73, 155303 (2006).

[2] Talapin, D. V. and Murray, C. B., "PbSe nanocrystal solids for $\mathrm{n}$-and p-channel thin film field-effect transistors," Sci. 310, 86-89 (2005).

[3] Yu, D., Wang, C. J. and Guyot-Sionnest, P., "n-type conducting CdSe nanocrystal solids," Sci. 300, 1277-1280 (2003).

[4] Buda, M., Iordache, G., Stancu, V. and Botila, T., "Characterization of high performance PbS photodetectors," J. Optoelectronics and Adv. Mater. 10 (2), 306-310 (2008).

[5] Jarosz, M. V., Porter, V. J., Fisher, B. R., Kastner, M. A. and Bawendi, M. G., "Photoconductivity studies of treated CdSe quantum dot films exhibiting increased exciton ionization efficiency," Phys. Rev. B 70, 195327 (2004).

[6] Konstantatos, G., Howard, I., Fischer, A., Hoogland, S., Clifford, J., Klem, E., Levina, L. and Sargent, E. H., "Ultrasensitive solution-cast quantum dot photodetectors," Nature, 442, 180-183 (2006).

[7] Oertel, D. C., Bawendi, M. G., Arango, A. C. and Bulovic, V., "Photodetectors based on treated CdSe quantum-dot films," Appl. Phys. Lett. 87, 213505 (2005).

[8] Sun, L. F., Choi, J. J., Stachnik, D., Bartnik, A. C., Hyun, B. R., Malliaras, G. G., Hanrath, T. and Wise, F. W., "Bright infrared quantum-dot light-emitting diodes through inter-dot spacing control, Nature Nanotechnol. 7, 369-373 (2012).

[9] Bertoni, C., Gallardo, D., Dunn, S., Gaponik, N. and Eychmuller, A., Fabrication and characterization of red-emitting electroluminescent devices based on thiol-stabilized semiconductor nanocrystals," Appl. Phys. Lett. 90, 034107 (2007).

[10] Coe, S., Woo, W. K., Bawendi, M. and Bulovic, V., "Electroluminescence from single monolayers of nanocrystals in molecular organic devices," Nature, 420, 800-803 (2002).

[11] Plum, E., Fedotov, V. A., Kuo, P., Tsai, D. P. and Zheludev, N. I., "Towards the lasing spaser: controlling metamaterial optical response with semiconductor quantum dots," Optics Express, 17(10), 8548-8551 (2009).

[12] Shevchenko, E. V., Talapin, D. V., Kotov, N. A., O'Brien, S. and Murray, C. B., "Structural diversity in binary nanoparticle superlattices," Nature, 439, 55-59 (2006).

Distribution Statement A. Approved for public release; distribution unlimited. 
[13] Shevchenko, E. V., Talapin, D. V., Murray, C. B. and O’Brien, S., "Structural characterization of self-assembled multifunctional binary nanoparticle superlattices," J. Am. Chem. Soc. 128, 3620-3637 (2006).

[14] Zhao, N., Osedach, T. P., Chang, L.Y., Geyer, S. M., Wanger, D., Binda, M. T., Arango, A. C., Bawendi, M. G. and Bulovic, V., "Colloidal PbS quantum dot solar cells with high fill factor," ACS Nano, 4 (7), 3743-3752 (2010).

[15] Gur, I., Fromer, N. A., Geier, M. L. and Alivisatos, A. P., "Air-stable all-inorganic nanocrystal solar cells processed from solution," Sci. 310, 462-465 (2005).

[16] Dai, Q., Wang, Y. N., Zhang, Y., Li, X. B., Li, R.W., Zou, B., Seo, J. T., Wang, Y. D., Liu, M. H. and Yu, W. W., "Stability study of PbSe semiconductor nanocrystals over concentration, size, atmosphere, and light exposure," Langmuir, 25 (20), 12320-12324 (2009).

[17] Sykora, M., Koposov, A. Y., McGuire, J. A., Schulze, R. K., Tretiak, O., Pietryga, J. M. and Klimov, V. I., "Effect of air exposure on surface properties, electronic structure, and carrier relaxation in PbSe nanocrystals," ACS Nano, 4, 2021-2034 (2010).

[18] Ihly, R., Tolentino, J., Liu, Y., Gibbs, M. and Law, M., "The photothermal stability of PbS quantum dot solids," ACS Nano, 5 (10), 8175-8186 (2011).

[19] Stouwdam, J. W., Shan, J. N. and van Veggel, F. C. J. M., "Photostability of colloidal $\mathrm{PbSe}$ and $\mathrm{PbSe} / \mathrm{PbS}$ Core/Shell Nanocrystals in solution and in the solid state," J. Phys. Chem. C 111 (3), 1086-1092 (2007).

[20] Zhao, H., Chaker, M. and Ma, D., "Bimodal photoluminescence during the growth of PbS quantum dots," J. Phys. Chem. C 113, 6497-6504, (2009).

[21] Hines, M. A. and Scholes, G. D., "Colloidal PbS nanocrystals with size-tunable nearinfrared emission: Observation of post-synthesis self-narrowing of the particle size distribution," Adv. Mater. 15, 1844-1849, (2003).

[22] Wang, J. S., Smetana, A. B., Boeckl, J. J., Brown, G. J. and Wai, C. M., "Depositing ordered arrays of metal sulfide nanoparticles in nanostructures using supercritical fluid carbon dioxide," Langmuir, 26(2), 1117-1123 (2010).

[23] Smetana, A. B., Wang, J. S., Boeckl, J. J., Brown, G. J. and Wai, C. M., "Deposition of ordered arrays of gold and platinum nanoparticles with an adjustable particle size and interparticle spacing using supercritical $\mathrm{CO}_{2}$," J. Phys. Chem. C 112, 2294-2297 (2008).

[24] Liu, J., Anand, M. and Roberts, C. B. "Synthesis and extraction of $\beta$-D-glucosestabilized Au nanoparticles processed into low-defect, wide-area thin films and ordered arrays using $\mathrm{CO}_{2}$-expanded liquids," Langmuir 22, 3964-3971 (2006).

[25] McLeod, M. C., Kitchens, C. L. and Roberts, C. B., " $\mathrm{CO}_{2}$-expanded liquid deposition of ligand-stabilized nanoparticles as uniform, wide-area nanoparticle films," Langmuir, 21, 2414-2418 (2005).

[26] Lin, X. M., Jaeger, H. M., Sorensen, C. M. and Klabunde, K. J., "Formation of longrange-ordered nanocrystal superlattices on silicon nitride substrates," J. Phys. Chem. B 105, 3353-3357 (2001).

[27] Ohara, P. C. and Gelbart, W. M., "Interplay between hole instability and nanoparticle array formation in ultrathin liquid films," Langmuir, 14, 3418-3424 (1998).

Distribution Statement A. Approved for public release; distribution unlimited. 
[28] Asunskis, D. J. and Hanley, L., "Valence band and core level x-ray photoelectron spectroscopy of lead sulfide nanoparticle-polymer composites", Surf. Sci. 601, 46484656 (2007).

[29] Zachary, A. M. Bolotin, I. L., Asunskis, D. J., Wroble, A. T. and Hanley, L., "Cluster beam deposition of $\mathrm{PbS}$ nanocrystals into organic matrices," ACS Applied Materials and Interfaces 1(8), 1770-1777 (2009).

[30] CasaXPS Version 2.3.15, Copyright (C) 1999-2009 Casa Software Ltd.

[31] Moulder, J. F., Stickle, W.F., Sobol, P. E. and Bomben, K.D., "Handbook of X-ray photoelectron spectroscopy," copyright 1992, the Perkin-Elmer Corporation, USA.

[32] Tarlov, M. J., Burgess, R. F. Jr., and Gillen, G., "UV photopatterning of alkanethiolate monolayers self-assembled on gold and silver," J. Am. Chem. Soc., 115, 5305 (1993).

[33] Wang, J., Ullrich, B. and Brown, G., "Lead sulfide quantum dot synthesis, deposition, and temperature dependence studies of the Stokes shift," 2011 MRS fall proceeding, Mater. Res. Soc. Symp. Proc. Vol.1409 2012 Materials Research Society

DOI:10:1557/opl.2012.755.

Distribution Statement A. Approved for public release; distribution unlimited. 


\subsection{PUBLICATIONS}

\subsection{Refereed Journal Articles}

"Purification of Single-walled Carbon Nanotubes using a Supercritical Fluid Extraction

Method", J. S. Wang, C. M. Wai, K. Shimizu, F. Cheng, John J. Boeckl, B. Maruyama, and G. J. Brown, J. of Physical Chemistry C 112, 2294 (2008).

"New Kronig-Penney equation emphasizing the band edge conditions", F. Szmulowicz, Eur. J. Phys. 29, 507 (2008).

"A New Fundamental Equation for Band Spectra of Dielectric Layer Films", F. Szmulowicz, J. Opt. A : Pure Appl. Opt. 10, 075205 (2008).

"Electronic Properties of p-GaAs deposited on n-Si with Pulsed-laser Deposition", B. Ullrich, A. Erlacher, H. E. Smith, W. C. Mitchel and G. J. Brown, J. Phys.: Condens. Matter 20, 175217 (2008).

"Deposition of Ordered Arrays of Gold and Platinum Nanoparticles with an Adjustable Particle Size and Interparticle Spacing Using Supercritical $\mathrm{CO}_{2}$ ", A. B. Smetana, J. S. Wang, J. J. Boeckl, G. J. Brown and C. M. Wai, J. Phys. Chem. C 112, 2294 (2008).

"Compositional Analysis of III-V Semiconductor Interfaces Using Exit-Plane Wave Functions in High-Resolution Transmission Electron Microscopy", K. Mahalingam, K. G. Eyink, G. J.

Brown, D. L. Dorsey, C. F. Kisielowski, and A. Thust, J. of Microscopy 320, 372 (2008).

"Study of Residual Background Carriers in Midinfrared InAs/GaSb Superlattices for Uncooled Detector Operation", H. J. Haugan, S. Elhamri, F. Szmulowicz, B. Ullrich, G. J. Brown and W. C. Mitchel, Appl. Phys. Lett. 92, 071102 (2008).

"Growth Optimization for Low Residual Carriers in Undoped Midinfrared InAs/GaSb Superlattices", H. J. Haugan, S. Elhamri, G. J. Brown and W. C. Mitchel, J. Appl. Phys. 104, 073117 (2008).

"Analysis of (110) Indium Arsenide-Gallium Antimonide Superlattices for Infrared Detection", F Szmulowicz, H. J. Haugan and G. J. Brown, J. Appl. Phys. 104, 074505 (2008).

"Terahertz Frequency Range Dielectric Tunability of $\mathrm{Pb}\left(\mathrm{Mg}_{1 / 3} \mathrm{Nb}_{2 / 3}\right) \mathrm{O}_{3}-\mathrm{PbTiO}_{3}$ ”, Y. Lu, R. J. Knize, K. Reinhardt, and G. J. Brown, Integrated Ferroelectrics 97, 3 (2008).

"Realization of Negative Refraction in Oxide Multilayers", Y. Lu, G. Brown and K. Reinhardt, Advances in OptoElectronics 8, 279 (2008), Special Issue on Progress in Domain Engineered Photonic Materials

"Dark Current and Band Profiles in Low Defect Density Thick Multilayered GaAs/InAs SelfAssembled Quantum Dot Structures for Infrared Detectors", T. Asano, A. Madhukar, K. Mahalingam and G. J. Brown, J. Appl. Phys. 104, 113115 (2008).

Distribution Statement A. Approved for public release; distribution unlimited. 
"Optimizing Residual Carriers in Undoped InAs/GaSb Superlattices for High Operating Temperature Mid-infrared Detectors”, H. J. Haugan, S. Elhamri, B. Ullrich, F. Szmulowicz, G. J. Brown and W. C. Mitchel, J. of Crystal Growth 311, 1897 (2009).

"Carrier Mobility as a Function of Carrier Density in Type-II InAs/GaSb Superlattices", F. Szmulowicz, S. Elhamri, H. J. Haugan, G. J. Brown and W. C. Mitchel, J. Appl. Phys. 105, 074303 (2009).

"Growth and Characterization of Carbon Nanopearls on a Silicon Substrate", S. Houston, G. J. Brown, T. Murray, S. Fairchild, K. Eyink, A. Smetana, J. Electronic Materials DOI 10.1007/s11664-009-0713-7 (2009).

"Room Temperature Photoluminescence of Amorphous GaAs", B. Ullrich and G. J. Brown, Materials Letts 63, 2489 (2009).

"Depositing Ordered Arrays of Metal Sulfide Nanoparticles in Nanostructures Using Supercritical Fluid Carbon Dioxide", J. S. Wang, A. B. Smetana, J. J. Boeckl, G. J. Brown and C. M. Wai, Langmuir 26, 1117 (2010).

"Colloidal Quantum Dot Absorption Enhancement in Flexible Fano Filters", L. Chen, H. Yang, Z. Qiang, H. Pang, L. Sun, Z. Ma, R. Pate, A. Stiff-Roberts, S. Gao, G. J. Brown and W. Zhou, Appl. Phys. Lett. 96, 083111 (2010).

"Study of the driving force for the self-assembly of heterojunction quantum dots (zero D molecules) using finite element analysis", K. G. Eyink, L Grazulis, M. Twyman, and K. Mahalingam, J. Vac. Sci. Technol. B 28, C3C33-C3C36 (2010).

"Photoluminescence of PbS quantum dots on Semi-insulating GaAs", B. Ullrich, X. Xiao and G. J. Brown, J. Appl. Phys. 108, 013525 (2010).

"Structure, Magnetic, and Microwave Properties of Thick Ba-hexaferrite Films Epitaxially Grown on GaN/ $\mathrm{Al}_{2} \mathrm{O}_{3}$ Substrates", Z. Chen, A. Yang, K. Mahalingam, K. L. Averett, J. Gao, G. J. Brown, C. Vittoria, and V. G. Harris, Appl. Phys. Lett. 96, 242502 (2010).

"Analysis of Thermal Band Gap Variations of PbS Quantum Dots by Fourier Transform Transmission and Emission Spectroscopy”, B. Ullrich, J. Wang and G. J. Brown, Appl. Phys. Lett. 99, 081901 (2011).

“Doping of GaAs by Laser Ablated ZnTe”, B. Ullrich and G. J. Brown, Appl. Phys. Lett. 99, 091103 (2011).

"Band Gap Formation in Graphene by In-situ Doping", J. Park, W. C. Mitchel, G. J. Brown, S. Elhamri, L. Grazulis, H. E. Smith, S. D. Pacley, J. J. Boeck1, K. G. Eyink, S. Mou, D. H. Tomich, and J. E. Hoelscher, Appl. Phys. Lett. 98, 203102 (2011).

"Free-Standing Arrays of Isolated $\mathrm{TiO}_{2}$ Nanotubes via Supercritical Fluid Drying", J. R. Deneault, X. Xiao, T. S. Kang, J. S. Wang, C. M. Wai, G. J. Brown, and M. F. Durstock, Chem PhysChem 13, 256 (2012).

Distribution Statement A. Approved for public release; distribution unlimited. 
"Phase Sensitive Detection of Photoluminescence with Fourier Transform Spectroscopy", B. Ullrich and G. J. Brown, Rev. of Sci. Instruments 83, 016105 (2012).

"Semiconductor Band Gap Localization via Gaussian Function", B. Ullrich and G. J. Brown, Semicond. Sci. \& Technol. 27, 105016 (2012).

"Photoluminescence Under High-electric Field of PbS Quantum Dots", B. Ullrich, J. S. Wang and G. J. Brown, AIP Advances 2, 042132 (2012).

"Supercritical Fluid Deposition, of Uniform PbS Nanoparticle Films for Energy Transfer Studies", J. S. Wang, G. J. Brown, W.-C. Hun, C. M Wai, Chem PhysChem 13, 2068 (2012).

"Nanofabricated Quantum Dot Array Formation through Annealing of Nanopatterned Planar InAs", K. G. Eyink, L. Grazulis, K. Mahalingam, J. Shoaf, V. Hart, D. Esposito, J. Hoelscher, M. Twyman and D. H. Tomich,J. Vac. Sci. Technol. B 30, 041806 (2012).

"Morphology and Energy Transfer in PbS Quantum Dot Arrays Formed with Supercritical Fluid Deposition", J. S. Wang, B. Ullrich, G. J. Brown and C. M. Wai, Mater Chem Physics 141, 195 (2013).

"Impact of Laser Excitation Intensity on the Photoemission of PbS Quantum Dots on GaAs", B. Ullrich and J. S. Wang, J. of Luminescence, 143, 645 (2013).

"All-optical Tuning of the Stokes Shift in PbS Quantum Dots", B. Ullrich and J. S. Wang, Appl. Phys. Lett. 102, 071905 (2013).

"Growth and Spectroscopic Ellipsometry Evaluation of Composite Layers of ErAs and InAs Nanoparticles", K. G. Eyink, L. J. Bissell, J. Shoaf, D. H. Tomich, D. Esposito, M. Hill, L. Grazulis, A. Aronow and K. Mahalingam, J. Vac. Sci. Technol. B 31, 03 C131 (2013).

"Nanocrystal - Semiconductor Interface: Atomic-Resolution Cross-sectional Transmission Electron Microscopy Study of Lead Sulfide Nanocrystal Quantum Dots on Crystalline Silicon", Z. Lingley, K. Mahalingham, S. Lu, G. J. Brown and A. Madhukar, Nano Research 7, 219 (2014). DOI 10.1007/s12274-013-0389-4

"Nanoparticle Film Deposition Using a Simple and Fast Centrifuge Sedimentation Method", J. S. Wang, A. Markelonis, C. M. Wai, and G. J. Brown, Applied Nanoscience (2014). DOI 10.1007/s13204-014-0338

\subsection{Conference Proceedings}

"Proposal for (110) InAs/GaSb Superlattices for Infrared Detection", F. Szmulowicz, H. J. Haugan, and G. J. Brown, Quantum Sensing and Nanophotonic Devices V, Rengarajan Sudharsanan and Christopher Jelen, Editors SPIE Proc. 6900, 69000L (2008).

"Interface Roughness Estimate from Carrier Transport in InAs/GaSb Superlattices", F. Szmulowicz, S. Elhamri, H. J. Haugan, G. J. Brown and W. C. Mitchel, Quantum Sensing and Nanophotonic Devices V, R.Sudharsanan and C. Jelen, Editors SPIE Proc. 6900, 69000P (2008).

Distribution Statement A. Approved for public release; distribution unlimited. 
"Fano Resonance Enhanced Infrared Absorption for Infrared Photodetectors", Z. Qiang, W. Zhou, M. Lu and G. J. Brown, Photonic Crystal Materials and Devices VII,Ali Adibi, ShawnYu Lin, Axel Scherer, Editors SPIE Proc. 6901, 69010F (2008).

"Plasma Enhanced chemical Vapor Deposition of High Refractive Index Polymer Films", J. O. Enlow, H. Jiang, K. G. Eyink, J. T. Grant, W. Su, W.E. Johnson, and T. Bunning, Linear and NonlinearOptics of Organic Materials VIII, SPIE Proc. 7049, 70490T (2008).

"Spectrally Selective Infrared Absorption Enhancement in Photonic Crystal Cavities", W. Zhou, H. Yang, Z. Qiang, L. Chen and G. J. Brown, Nano- and Macro- Photonics for Space Environments II, Edward W. Taylor, David A. Cardimona, Editors, SPIE Proc. 7095, 709507 (2008).

Characteristics of surface-normal Fano Filters on Flexible Plastic Substrates", Z. Qiang, H. Yang, L. Chen, H. Pang, Z. Ma, W. Zhou and G. J. Brown, Proceedings of the $20088^{\text {th }}$ IEEE Conference on Nanotechnology (NANO), pp. 78-80 (2008).

"Angle and Polarization Dependent Characteristics of Colloidal Quantum Dot Absorption in Fano Filters on Flexible Substrates”, L. Chen, H. Yang, Z. Qiang, H. Pang, Z. Ma, J. Xu, G. J. Brown and W. Zhou, Quantum Sensing and Nanophotonic Devices VI, SPIE Proc. 7222, 72220V (2009).

"Control of Residual Background Carriers in Undoped Mid-infrared InAs/GaSb Superlattices", H. J. Haugan, S. Elhamri, W. C. Mitchel, B. Ullrich, G. J. Brown, L. Grazulis and S. Houston, Quantum Sensing and Nanophotonic Devices VI, SPIE Proc. 7222, 72220Y (2009).

"A Fundamental Equation for the Band Spectra of Dielectric Layer Films", F. Szmulowicz, Photonic and Phononic Crystal Materials and Devices VIII, SPIE Proc. 7223, 72230S (2009).

"Morphological Change in Tip Based Nano patterned Planar InAs", K. G. Eyink, J. Shoaf, and L. Grazulis, Quantum Dots, Particles, and Nanoclusters VI, K. Eyink, F. Szmulowicz and D. Huffaker, Editors, SPIE Proc. 7224, 722408 (2009).

"GaSb Substrates with Extended IR Wavelength for Advanced Space Based Applications", L.P. Allen, P. Flint, G. Dallas, D. Bakken, K. Blanchat, G. J. Brown, S.R. Vangala, W.D. Goodhue and K. Krishnaswami , Infrared Technology and Applications XXXV, SPIE Proc. 7298, 72983P (2009).

"Depositing Ordered Arrays of Metal Sulfide Nanoparticles in Nano-structures Using Supercritical Fluid Carbon Dioxide", J. S. Wang, A. B. Smetana, C. M. Wai, J. J. Boeckl, and G. J. Brown, Large-Area Processing and Patterning for Optical, Photovoltaic, and Electronic Devices II, Mater. Res. Soc. Symp. Proc. 1196, C06-23 (2010).

"Direct Measurement of Spectrally Selective Absorption Enhancement in Fano Resonance Photonic Crystal Cavities on Plastic Substrates", L. Chen, H. Yang, Z. Qiang, L. Sun, Z. Ma, J. $\mathrm{Xu}, \mathrm{R}$. Pate, A. Stiff-Roberts, G. J. Brown, W. Zhou, Photonic and Phononic Crystal Materials and Devices X, Ali Adibi, Shawn-Yu Lin, Axel Scherer, Editors, SPIE Proc. 7609, 76090E (2010).

Distribution Statement A. Approved for public release; distribution unlimited. 
"Shape Changes in Patterned Planar InAs as a Function of Thickness and Temperature", K. G. Eyink, L. Grazulis, K. Mahalingam, M. Twyman, J. Shoaf, J. Hoelscher, C. Clafin and D.

Tomich, Quantum Dots and Nanostructures: Synthesis, Characterization and Modeling VII, K. Eyink, F. Szmulowicz and D. Huffaker, Editors, SPIE Proc. 7610, 761000 (2010).

"Lead Sulfide Quantum Dot Synthesis, Deposition, and Temperature Dependence Studies of the Stokes Shift", J. S. Wang, B. Ullrich and G. J. Brown, Functional Semiconductor Nanocrystals and Metal-Hybrid Structures, Cammy R. Abernathy, Paul V. Braun, Masashi Kawasaki and Kathryn J. Wahl, Editors, MRS Proc. 1409 (2012).

"PbS nanoparticles: Synthesis, Supercritical Fluid Deposition and Optical Characterization", J. S. Wang, B. Ullrich, and G. J. Brown, Solution Synthesis of Inorganic Films and Nanostructured Materials, MRS Proc. 1449 (2012).

"Spectral Selective Absorption Enhancement from Stacked Ultra-thin InGaAs/Si Fano Resonance Nanomembranes", A. S. Chadha, W. Yang, T. K. Saha, S. Chuwongin, W. Zhou, Z. Ma and G. J. Brown, Quantum Sensing and Nanophotonic Devices IX, M. Razeghi, E. Tourné \& G. J Brown, Editors, SPIE Proc. 8268, 82680G-1 (2012).

"Fourier Spectroscopy on PbS Quantum Dots", B. Ullrich, J. S. Wang, X. Y. Xiao and G. J. Brown, Quantum Dots and Nanostructures: Synthesis, Characterization, and Modeling IX, Kurt Eyink, Diana Huffaker and Frank Szmulowicz, Editors, SPIE Proc. 8271 (2012).

"Stability Studies of Lead Sulfide Colloidal Quantum Dot Films on GaAs", J. S. Wang, E. S. Steenbergen, H. E. Smith, L. Grazulis, B. Ullrich and G. J. Brown, Quantum Dots and Nanostructures: Synthesis, Characterization and Modeling X, Kurt G. Eyink, Diana L. Huffaker, and Frank Szmulowicz, Editors, SPIE Proc. 8634, 86340T (2013).

"Emission of Precipitation Deposited PbS Quantum Dots on Polyethylene Terephthalate", B. Ullrich, A. Markelonis, J. S. Wang, G. J. Brown, Advanced Materials and Processes for “Systems-on-Plastic”, MRS Proc. 1436, (2013). DOI:10.1117/opl.2013.1096.

Distribution Statement A. Approved for public release; distribution unlimited. 


\section{LIST OF ACRONYMS, ABBREVIATIONS, AND SYMBOLS}

\begin{tabular}{|c|c|}
\hline Acronym & Definition \\
\hline InAs & Indium Arsenide \\
\hline $\mathrm{GaSb}$ & Gallium Antimonide \\
\hline GaAs & Gallium Arsenide \\
\hline $\mathrm{PbS}$ & Lead Sulfide \\
\hline $\mathrm{CO}_{2}$ & Carbon Dioxide \\
\hline SL & Superlattice \\
\hline IRS & Interface Roughness Scattering \\
\hline S-R & Shockley-Read \\
\hline MBE & Molecular Beam Epitaxy \\
\hline $\mathrm{T}_{\mathrm{g}}$ & Growth Temperature \\
\hline PL & Photoluminescence \\
\hline FWHM & Full Width at Half Maximum \\
\hline MWIR & Mid Wavelenght Infrared \\
\hline MBE & Molecular Beam Epitaxy \\
\hline VLWIR & Very Long Wavelength Infrared \\
\hline SIMS & Secondary Ion Mass Spectroscopy \\
\hline HRXRD & High Resolution X-Ray Diffraction \\
\hline HRTEM & High Resolution Transmission Electron Miscroscopy \\
\hline $\mathrm{PC}$ & Photoconductivity \\
\hline PR & Photoresponse \\
\hline PSD & Phase Sensitive Detection \\
\hline PSE & Phase Sensitive Excitation \\
\hline FTIR & Fourier Transform Infrared \\
\hline SRS & Stanford Research Systems \\
\hline $\mathrm{DC}$ & Direct Current \\
\hline TEM & Transmission Electron Microscope \\
\hline QDIP & Quantum Dot Infrared Photodetector \\
\hline QDs & Quantum Dots \\
\hline SEM & Scanning Electron Microscope \\
\hline $\mathrm{CW}$ & Continuous Wave \\
\hline sc- $-\mathrm{CO}_{2}$ & Supercritical Fluid $\mathrm{CO}_{2}$ \\
\hline ODE & Octadecene \\
\hline TMS & bis(trimethylsilyl)sulfide \\
\hline TOP & Trioctylphosphine \\
\hline OA & Oleic Acid \\
\hline
\end{tabular}

Distribution Statement A. Approved for public release; distribution unlimited. 


\section{LIST OF ACRONYMS, ABBREVIATIONS, AND SYMBOLS (Cont'd)}

\begin{tabular}{|c|c|}
\hline Acronym & Definition \\
\hline FEG & Field Emission Gun \\
\hline XRD & X-ray Diffraction \\
\hline GAS & Gas-Antisolvent \\
\hline OA & Optical Absorbance \\
\hline PINC & Polymer-Inorganic Nanocomposite \\
\hline PET & Polyethylene Terephthalate \\
\hline LO & Longitudinal Optical \\
\hline XPS & X-ray Photoelectron Spectroscopy \\
\hline AFM & Atomic Force Microscopy \\
\hline S.I. & Semi-Insulating \\
\hline
\end{tabular}

